U.S. Department

Of Transportation

Federal Highway

Administration

\title{
SYMBOL SIGNING DESIGN FOR OLDER DRIVERS
}

Research and Development

Turner-Fairbank Highway Research Center 6300 Georgetown Pike McLean, VA 22101-2296 


\section{Preface}

There has been an increasing use of symbols, including those on highway signs, over the past 2 decades. At the same time, there has been a rapid growth in both the numbers and proportions of older drivers. Research on symbols has shown that many symbols are poorly understood and/or difficult to recognize at a distance, especially by older drivers. In spite of the considerable research on traffic sign symbols, there has not yet been a thorough evaluation of all symbols used on U.S. highways.

This report presents a review of the literature on information processing abilities of older drivers and human factors research on traffic sign symbols. It describes a series of studies, surveys, and laboratory experiments that examined symbols in the U.S. Manual on Uniform Traffic Control Devices (MUTCD). Initially all symbols in the Manual were evaluated for comprehension and daytime legibility distance among drivers of all ages. This was followed by evalation of a set of 18 symbols using measures of nighttime legibility (with and without glare), reaction time, glance legibility, and conspicuity. Older drivers were found to have poorer understanding of the symbols, as well as shorter legibility distances, higher glance legibility thresholds, reaction times, and conspicuity search times. Glare was found to reduce legibility of signs only for older drivers.

Modifications and redesigns to 13 of the symbols resulted in better understanding of 3 symbols and increased legibility of 11 new designs. Understanding and legibility of five novel symbols which were developed for this project were found to be comparable to those of the redesigned symbols.

Recommendations were made for changes to specific symbols and guidelines were proposed for the design of symbol traffic signs. A proposal was made for the use of design optimization techniques, using computer, in the development and evaluation of signs. 


\begin{tabular}{|c|c|}
\hline 2. Government Accession No. & 3. Recipient's Catalog No. \\
\hline 4. Title and Subtitle & $\begin{array}{l}\text { 5. Report Date } \\
\text { July } 1997 \\
\end{array}$ \\
\hline SYMBOL SIGNING DESIGN FOR OLDER DRIVERS & 6. Performing Organization Code \\
\hline $\begin{array}{l}\text { 7. Author(s) Robert Dewar, Donald Kline, Frank Scheiber, } \\
\text { a n d Allen Swan o n }\end{array}$ & 8. Performing Organization Report No. \\
\hline 9. Performing Organization Name and Address & 10. Work Unit No. (TRAIS) \\
\hline $\begin{array}{l}\text { Swanson Transportation Consultants, Ltd. } \\
\text { P.O. Box 83001, Canyon Meadows Post Office } \\
\text { Calgary, AB Canada, T2W 6G8 } \\
\end{array}$ & $\begin{array}{l}\text { 11. Contract or Grant No. } \\
\text { DTFH61-01-C-00018 }\end{array}$ \\
\hline $\begin{array}{l}\text { 12. Sponsoring Agency Name and Address } \\
\text { Office of Safety and Traffic Operations R \& D } \\
\text { Federal Highway Administration } \\
6300 \text { Georgetown Pike, McLean, VA 22101-2296 }\end{array}$ & $\begin{array}{l}\text { 13. Type of Report and Period Covered } \\
\text { Final technical report } \\
\text { June, } 1991 \text { - June, } 1994\end{array}$ \\
\hline \multicolumn{2}{|l|}{ 15. Supplementary Notes } \\
\hline Contracting Officer's Technical Representative (COTR): & $\begin{array}{r}\text { Dr. Truman Mast } \\
\text { HSR-30 }\end{array}$ \\
\hline
\end{tabular}

16. Abstract

This project evaluated the effectiveness of symbol traffic signs for young, middle-aged and elderly drivers. Daytime legibility distance and comprehension of 85 symbols in the $M$ anual on Uniform Traffic Control Devices (MUTCD) were measured. Legibility distance under night and night-with-glare conditions, glance legibility, reaction time and conspicuity were measured for 18 of these symbols. Selected symbols were modified or redesigned and five novel symbols were designed, using an image-processing iterative filter-redesign approach. These new signs were evaluated on measures of comprehension and legibility distance under day, night and night-with-glare conditions. Of the 85 symbols many were we11 understood, but 10 were understood by fewer than $40 \%$ of drivers. Increased driver age was associated with lower comprehension levels and legibility distances (under all test conditions), as well as higher glance legibility thresholds, reaction times and conspicuity search times. Modifications and redesigns to the symbols resulted in better understanding of three messages and improved daytime legibility distances of 11 of the new signs. Glare reduced legibility of the signs only for the elderly drivers.

Recommendations were made for changes to specific symbol signs. Guidelines were proposed for the design of symbol traffic signs, and a proposal was made for design optimization techniques to be employed in the future development and evaluation of such signs.

\section{Keywords}

symbols, traffic signs, comprehension, Legibility distance, glance legibility, reaction time, conspicuity, older drivers, night legibility, glare
18. Distribution Statement

No restrictions. This document is available to the public through the National Technical Information Service. Springfield, VA 22161

\begin{tabular}{|c|c|}
\hline $\begin{array}{c}\text { 19. Security Classif. (of this report) } \\
\text { Unclassified }\end{array}$ & $\begin{array}{c}\text { 20. Security Classif. (of this page) } \\
\text { Unclassified }\end{array}$
\end{tabular}




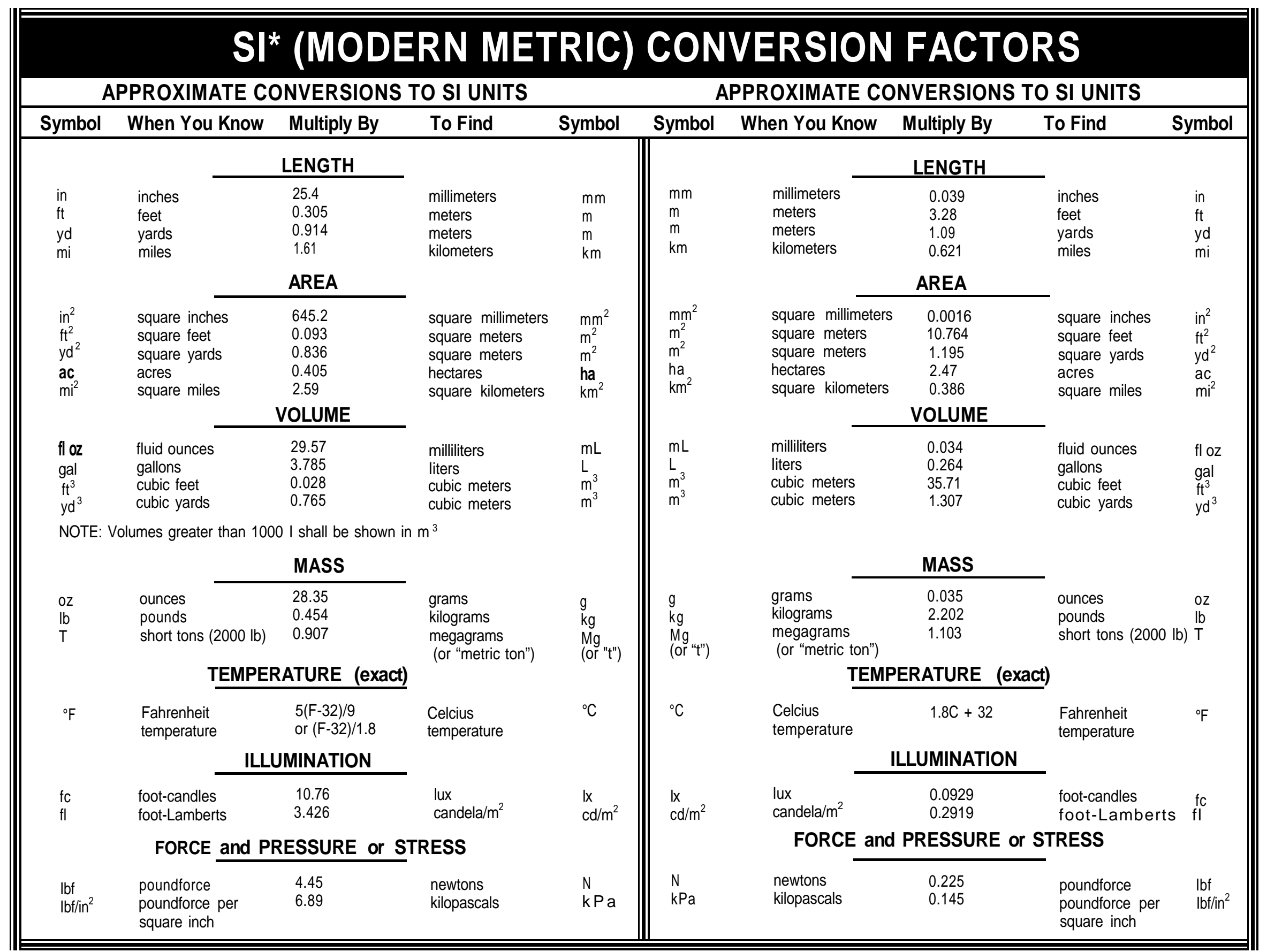

${ }^{*} \mathrm{SI}$ is the symbol for the International System of Units. Appropriate

rounding should be made to comply with Section 4 of ASTM E380. 


\section{TABLE OF CONTENTS}

Section

CHAPTER 1: DRIVER AGE AND SYMBOL SIGN EFFECTIVENESS

CHAPTER 2: LITERATURE REVIEW

AGING AND DRIVING

Demographics Of Older Drivers

Accident Rates

Increased Vulnerability To Injury Among

The Elderly

SYMBOL SIGN DESIGN

A Brief History Of Traffic Sign Symbols

The Advantages Of Symbols

HIGHWAY SIGN EFFECTIVENESS

Criteria For Traffic Sign Design

Factors Determining Highway Sign Effectiveness

Environmental Determinants Of Sign Effectiveness

SYMBOL SIGN DESIGN AND EVALUATION

Traditional Methods

Emerging Technologies

Standards For Symbol Effectiveness

SENSORY, PERCEPTUAL, COGNITIVE, AND

PSYCHOMOTOR AGING

Age-Related Sensory, Perceptual Cognitive, And

Psychomotor Change

The Eye

Sensitivity To Light

Spatial Resolution

Spatiotemporal Resolution

Attention, Cognition, And Psychomotor Change

AGING AND HIGHWAY SIGN EFFECTIVENESS

Aging And Sign Luminance

Aging And Symbol Signs

Aging And Sign Design

CHAPTER 3: DAYTIME LEGIBILITY DISTANCE OF SYMBOL HIGHWAY SIGNS 35

METHOD

Subjects

Stimuli And Apparatus

Procedure

RESULTS

Contrast Sensitivity

Sign Legibility Distance

Visual Predictors Of Sign Legibility

DISCUSSION 
TABLE OF CONTENTS (continued)

Section

CHAPTER 4: COMPREHENSION OF STANDARD SYMBOL SIGNS

FOCUS GROUP STUDY

METHOD

Subjects

Procedure

RESULTS

DISCUSSION

COMPREHENSION STUDY

METHOD

60

Subjects

Stimuli And Apparatus

60

Procedure

60

61

61

RESULTS

63

DISCUSSION

COMBINING DATA FROM COMPREHENSION AND

LEGIBILITY DISTANCE

CHAPTER 5: NIGHTTIME LEGIBILITY OF STANDARD SYMBOL HIGHWAY SIGNS

METHOD

Subjects

Stimuli And Apparatus

79

Procedure

RESULTS

Contrast Sensitivity

Sign Legibility Distance

Visual Predictors of Sign Legibility

DISCUSSION

CHAPTER 6: NIGHTTIME LEGIBILITY OF STANDARD SYMBOL HIGHWAY SIGNS IN THE PRESENCE OF PERIPHERAL GLARE

METHOD

Subjects

Stimuli And Apparatus

91

Procedure

RESULTS

Contrast Sensitivity

Sign Legibility Distance

Visual Predictors Of Sign Legibility 97

DISCUSSION 
Section

CHAPTER 7: DAYTIME GLANCE LEGIBILITY AND REACTION TIME FOR SYMBOL HIGHWAY SIGNS AS A FUNCTION OF DRIVER AGE

METHOD

Subjects

Stimuli And Apparatus

106

Procedure

RESULTS

108

Contrast Sensitivity

108

Glance Legibility

Reaction Time

Relationships Between Visual Measures And

Glance Legibility Or Reaction Time

DISCUSSION

Glance Legibility

Reaction Time

Visual Functioning, Glance Legibility, And

Reaction Time

Relationships Among Measures Of Sign

Effectiveness

CHAPTER 8: CONSPICUITY OF SYMBOL SIGNS

METHOD

Subjects

Stimuli And Apparatus

Procedure

RESULTS

Contrast Sensitivity

Search Time

Response Accuracy

Relationships Between Visual Measures And

Relationships Between Conspicuity And Other

Measures of Sign Effectiveness

Conspicuity

Conspicuity And Visual Functioning

Relationships Between Conspicuity And Other Measures 
CHAPTER 9: DEVELOPMENT OF ALTERNATIVE AND NOVEL SYMBOL SIGNS

MODIFICATION OF SIGNS

REDESIGN OF SIGNS

DESIGN OF NOVEL SIGNS

CHAPTER 10: COMPREHENSION OF MODIFIED, REDESIGNED, AND NOVEL SYMBOLS

FOCUS GROUP STUDY

METHOD

Subjects

Procedure

RESULTS

COMPREHENSION

METHOD

Subjects

Stimuli And Apparatus

Procedure

RESULTS AND DISCUSSION

150

Familiarity Of Novel Symbols

CONCLUSIONS

CHAPTER 11: DAYTIME LEGIBILITY OF MODIFIED, REDESIGNED, AND NOVEL HIGHWAY SIGNS

METHOD

Subjects

Stimuli And Apparatus

Procedure

RESULTS

Contrast Sensitivity

Sign Legibility Distance

Visual Predictors Of Sign Legibility 167

Low-Pass Threshold And Sign Legibility 174

Sign Comprehension

Predictors Of Sign Comprehension

Sign Effectiveness And Driver Age

Age And Measures Of Spatial Vision

Visual Predictors Of Legibility 
TABLE OF CONTENTS (continued)

$\underline{\text { Section }}$

Page

CHAPTER 12: NIGHTTIME LEGIBILITY OF MODIFIED, REDESIGNED, AND NOVEL SYMBOL HIGHWAY SIGNS

METHOD

Subjects

183

Stimuli And Apparatus

Procedure

RESULTS

Contrast Sensitivity

Sign Legibility Distance

184

Visual Predictors Of Sign Legibility

188

Daytime Versus Nighttime Legibility

DISCUSSION

CHAPTER 13: NIGHTTIME LEGIBILITY OF MODIFIED, REDESIGNED, AND NOVEL SYMBOL HIGHWAY SIGNS IN THE PRESENCE OF PERIPHERAL GLARE

METHOD

Subjects

Stimuli And Apparatus

Procedure

RESULTS

Contrast Sensitivity

Sign Legibility Distance

Predictors of Sign Legibility

DISCUSSION

CHAPTER 14: RECOMMENDED CHANGES TO STANDARD SYMBOL SIGNS AND SYMBOL SIGN DESIGN GUIDELINES

RECOMMENDED CHANGES TO STANDARD SYMBOL SIGNS

Consistency In Design Of Common Elements

RECOMMENDATIONS TO ENHANCE THE COMPREHENSION OF STANDARD SYMBOL SIGNS

Recommended Changes To Regulatory Signs 205

Recommended Changes To Warning Signs 205

Recommended Changes To Guide Signs 206

Recommended Changes To Recreational/

Cultural Signs

Recommended Changes To School Signs 207

Driver Education 
TABLE OF CONTENTS (continued)

Section

Page

CHAPTER 14: RECOMMENDATIONS TO ENHANCE THE LEGIBILITY

DISTANCE OF STANDARD SYMBOL SIGNS 208

Design Constraints 208

General Changes 208

Regulatory Signs $\quad 209$

Warning Signs $\quad 209$

Guide Signs 210

Recreational/Cultural Signs $\quad 211$

SYMBOL SIGN DESIGN GUIDELINES 213

Criteria For Design $\quad 214$

Design Constraints 214

RECOMMENDATIONS TO ENHANCE COMPREHENSION OF SYMBOL SIGNS 214

Critical Vs. Educational Features $\quad 214$

Symbol Complexity 215

Depiction Of Human Figures 215

Depiction of Vehicles 215

Use Of Ahead Arrow 216

Confusions Within Classes Of Signs 216

Uniformity Of Design 217

Standards For Comprehension Levels 217

RECOMMENDATIONS TO ENHANCE LEGIBILITY DISTANCE

OF SYMBOL SIGNS 218

Legibility (Legibility Distance) 218

Symbol Details 218

Common Sign Features $\quad 219$

Specific Symbol Elements $\quad 219$

Driver Age, Lighting, And Visual Functioning 219

Standards For Legibility Distance 220

Legibility Guidelines 220

Enhancing Legibility Through Low-Pass Image Filtering 221

GLANCE LEGIBILITY 222

REACTION TIME 222

CONSPICUITY 222

A SEQUENTIAL-COMPONENT APPROACH TO SIGN DESIGN AND MODIFICATION 223

Methodology For Development Of Symbols 223 
TABLE OF CONTENTS (continued)

$\underline{\text { Section }}$

APPENDIX A FHWA SYMBOL HIGHWAY SIGNS

APPENDIX B SAMPLE CONSENT FORM

APPENDIX C DRIVER EXPERIENCE QUESTIONNAIRE

APPENDIX D LEGIBILITY DISTANCE SCORING KEY

APPENDIX E COMPREHENSION SCORING KEY

APPENDIX F 18 FHWA SYMBOL SIGNS FOR NIGHTTIME LEGIBILITY, GLANCE LEGIBILITY/REACTION TIME, AND CONSPICUITY STUDIES

APPENDIX G RESPONSE LATENCY: TEST SIGNS AND FOILS

APPENDIX H 19 FHWA MODIFIED, REDESIGNED, AND NOVEL SYMBOL SIGNS

APPENDIX I COMPREHENSION SCORING KEY FOR NOVEL SYMBOLS

APPENDIX J LEGIBILITY SCORING KEY FOR MODIFIED, REDESIGNED, AND NOVEL SYMBOL SIGNS 


\section{CHAPTER 1: DRIVER AGE AND SYMBOL SIGN EFFECTIVENESS}

Due to the rapidly increasing numbers of older drivers in recent years, there has been considerable interest in the older road user. This is evidenced by the number of conferences and workshops on this topic, the numerous publications on older drivers and pedestrians, and the amount of funding available for related research. Perhaps the single most important of these efforts has been the 2-day symposium sponsored by the Transportation Research Board (TRB) on "Transportation in an Aging Society: Improving Mobility and Safety of Elderly Persons," in 1987. A two-volume report presents the details of this symposium, a series of excellent reviews on a number of topics relevant to the older road user. ${ }^{(1,2)}$

The research literature indicates that accident rates increase from about age 55 on. One of the leading contributors to this is failure to heed signs. Previous research has shown that older drivers have difficulty detecting, reading, and understanding symbol traffic signs. There are a number of possible reasons for the difficulties encountered by older drivers, including their reduced ability to take in and process visual information due to limitations in the older visual system, problems in the division of attention required for the driving task, and their lack of familiarity with many of the symbols on today's highways.

Since the introduction of large numbers of symbols on traffic signs in the United States over 2 decades ago, there has been concern about how well drivers understand their meanings. Research on this topic has shown that many symbols are poorly understood and difficult to identify from a distance, especially among older drivers. Although there have been numerous studies of traffic sign symbols, most have evaluated a single variable, such as comprehension, reaction time or legibility distance. None has systematically examined symbols using several measures of effectiveness. That is, there has never been a thorough evaluation of all or most of the standard symbols used on U.S. highways. There is clearly a need for such an evaluation.

The objectives of this project were to:

1. Determine the effectiveness of symbol signs in the Manual on Uniform Traffic Control Devices (MUTCD), including those that are problematic for the driving population as a whole and for older drivers in particular. ${ }^{(3)}$

2. Develop and test alternative symbol signs that varied in their effectiveness and to make recommendations to improve them.

3. Investigate individual elements of symbol signs, to determine which elements, individually and in combination, are critical to effective sign design.

4. Develop sign design guidelines, that respond to the sensory, psychomotor and cognitive capabilities of all drivers, including older ones, to be used in current and future sign activities.

The project, conducted in two phases, involved male and female licensed drivers in three age groups-young (18 to 39 ), middle-aged (40 to 59), and older (60+). In phase 1, laboratory studies were conducted on daytime legibility of symbols and a survey was carried out to determine the levels of driver comprehension of 85 symbols in the 1988 MUTCD. On the basis 
of these findings a number of additional measures-legibility distance under night, and nightwith-glare conditions, glance legibility, reaction time, and conspicuity -- were taken on a set of 18 of these symbols selected to represent the Best, Intermediate, and Worst symbols from the first two studies. In phase 2 of the project, 13 of these symbols were either modified or redesigned in an effort to improve their legibility and understandability. In addition, five novel symbol messages were designed. These symbols were then tested for their comprehension and legibility distance under daytime, nighttime, and nighttime-with-glare conditions. The results served as the basis for recommended changes to current traffic sign symbols and the development of guidelines for symbol sign design. 


\section{CHAPTER 2: LITERATURE REVIEW}

\section{AGING AND DRIVING}

\section{Demographics Of Older Drivers}

The numbers, as well as the proportion, of older people (typically defined as those over 65) have increased dramatically in the United States over the past few decades and will continue to increase for some time to come.(l) Rosenbloom cites data indicating that the percentage of people in the United States over 70 who had drivers licenses doubled from the early 1950's to 1984. ${ }^{(4)}$ The increase has been especially rapid among women. In 1951 through 1956 only 8 percent of women over 70 were licensed to drive, while in 1984 this figure was 43 percent. People 65 and over have 18 percent of drivers licenses in the United States but do only 6 percent of the driving-mainly short trips and mainly during daylight. ${ }^{(4)}$ There is reason to believe that the proportion of older drivers will continue to increase, as people in our society are very dependent on personal transportation and are unlikely to give up readily the use of their automobiles.

\section{Accident Rates}

Traffic accident data partitioned on the basis of age have systematically shown that fatality rates-per million population, per million licensed drivers, or per million mi driven -- are highest for young (under 25) and old drivers (over 65). ${ }^{(5,6)}$ Due to their relatively low numbers and reduced amount of driving, the actual numbers of fatalities are a decreasing function of age and are very low for older drivers. ${ }^{(6)}$ There is evidence, however, that future cohorts of older drivers will not curtail their driving to the same extent. Travel surveys taken from 1969 to 1983 show that miles driven by drivers 65 and older have increased steadily. This is to be expected, since today's drivers have lived in the automobile era and will not readily accept reduced mobility.

Urban accidents are more common than rural ones, a difference that is greatest for drivers over 70. The same age trend is true for intersection, as compared with nonintersection, accidents and for multivehicle crashes, as compared with single-vehicle accidents. ${ }^{(7,8)}$ Older drivers are more likely to crash in daylight, which no doubt reflects their reduced rate of nighttime driving. $(9,10,11)$ All these differences may be due to the fact that older drivers are more likely to be driving in these situations/locations.

Consistent with their elevated rate of multivehicle accidents, older drivers are particularly likely to be involved in right-of-way accidents. Specifically, older drivers are more often involved in accidents that are based on failure to yield right-of-way, turn properly, or heed signs. ${ }^{(5,12)}$ Left-turn accidents are particularly common among older drivers. ${ }^{(13)}$ Accidents of the following types appear to be especially age-related: failure to yield to an oncoming vehicle when turning left at an intersection, failure to yield to approaching vehicles when entering or crossing a roadway at locations other than intersections, and failure to yield to a vehicle approaching from the right at an intersection. ${ }^{(13,14)}$ The general U-shaped relationship between age and traffic accident rates occurs for male and female drivers, under both day and night conditions, and at signalized intersections. ${ }^{(13)}$ 


\section{Increased Vulnerability To Injury Among The Older Driver}

Research on traffic injuries, whether to vehicle occupants or pedestrians, has shown that the older driver are much more vulnerable to serious injury and death, partly because they are physically fragile, have more difficulty recovering from injuries, and are more seriously injured, as compared with younger adults or children, in crashes of comparable severity. Evans has calculated the "fatality risk from similar physical insults," based on data from the Fatal Accident Reporting System (FARS), using the double pair comparison method. ${ }^{(6)}$ The fatality risk, relative to 20 -year-old males, increases steadily from age 20 upward at a fairly uniform rate of 2.3 percent per year for males and 2.0 percent for females. This risk is about three times as great at age 70 as at age 20. Females between the ages of 10 and 60 in similar crashes are more likely to die than are males; thereafter males are slightly more likely to die. The greatest gender difference never exceeds 35 percent.

Mackay has examined the issue of injury to older road users and reports that, of a sample of over 17 million people involved in traffic accidents, those 65 and over represented 5.0 percent, but 8.3 percent of the seriously injured and 10.5 percent of the fatally injured. ${ }^{(15)}$ In addition, the mortality rate for three specific Injury Severity Scores (scores of 20, 30, and 40) was strongly age dependent, especially for those over 50. Days spent in hospital following traffic injuries was also much greater for older accident victims. Mackay also observes that a given impact will produce more fractures, as well as more serious injury generally, for older persons than for young ones. This greater vulnerability makes it difficult to draw solid conclusions about the safety of older vehicle occupants and pedestrians, as compared with younger ones.

\section{SYMBOL SIGN DESIGN}

\section{A Brief History Of Traffic Sign Symbols}

A number of terms have been used to designate signs of the type that this report will refer to as "symbol." Strictly speaking, a symbol is something that is used to represent something else---it can come in a variety of forms and involve any of the human senses, not just vision. Spoken words, for example, are symbols. For purposes of this report, however, symbol signs will refer to those that communicate their messages using an icon or graphical presentation rather than text or words. The other terms often used to designate signs of this type are "pictograph," "glyph," and "icon." The use of symbols has increased dramatically over the past 2 decades, with the rapid increase in international travel and trade, and the resulting need to communicate quickly and accurately to those who do not understand the written and spoken language of a particular nation.

The history of traffic sign symbols goes back to the beginning of the century in Europe. The Convention on the International Circulation of Motor Vehicles, held in Paris in 1909, recommended four road signs warning of typical hazards of that time-hump, curve, road crossing, and railroad crossing. This recommendation was adopted by many European countries. However, since the signs were installed by private organizations, with the help of commercial sponsors, signs were often cluttered with advertising messages, a problem that remains in some countries. In 1926 the Convention Relative to Motor Traffic described a modest system of six signs, including pictographs for uneven carriage way and curve, and adopted the triangular sign 
shape to depict danger. The Convention for the Unification of Road Signs was adopted in Geneva, and the number of signs specified increased from 6 to 26, divided into three categories-danger signs, signs giving instructions, and information signs. ${ }^{(16)}$

A committee of the League of Nations, in 1939, recommended further refinements to the international road sign system, but World War II prevented their implementation. However, in 1949 a new "protocol on road signs," developed by the United Nations (U. N.) and specifying more than 50 signs, was signed by approximately 30 countries, mainly in Europe and Asia. Although this system is often referred to as the "international sign system," its use is by no means worldwide. In fact, even the European countries where it is used have a number of symbols unique to their national roadway systems. The U. N. convention was revised in 1953 and again in 1968. The 1953 version was adopted by many South American countries. In the 1960's Canada adopted a system of symbols based largely on the 1953 U. N. convention, with some of its own design as well. The United States sign system consisted mainly of word signs until 1970, when it adopted a new system that included many of the 1968 U. N. symbols.

In Japan, the "Ordinance Concerning Road Warning Signs and Road Direction Signs" was enacted in 1922 and became that country's first legal system for traffic signs. They were modeled after the British system and were mainly word signs. ${ }^{(17)}$ The system was modified in 1942, based on German signs of that era, and included a few symbols. Some of the 1949 U. N. symbols were adopted in 1950, but considerable use was made of signs consisting of both words and symbols, much like the U. S. system as it existed at that time. Even though international efforts at uniformity of traffic signs had been ongoing for many decades, a number of different signing systems were still in existence by the early 1970's.

\section{The Advantages of Symbols}

There are a number of advantages of symbols over word messages. They can be identified at a greater distance. ${ }^{(18,19)}$ They can also be identified more rapidly, and more accurately when seen at a glance; they are seen better under adverse viewing conditions. ${ }^{(19,20)}$ They can also be understood by people who do not read the language of the country in which they are used.

The major problem with symbols is that their meanings are not always obvious to the viewer. Although many are relatively easy to understand (e.g., "No Left Turn"), others present problems, even for experienced drivers. In spite of the fact that it is standard practice in many countries to place an "educational tab" with any new symbol for several years after its introduction into the system, it appears that many symbols are still not well understood by the driving public. It is clear that greater effort needs to be made by traffic control authorities to design easily understood symbols and to educate the public about their meanings.

An interesting psychological/philosophical question arises in connection with some types of symbol messages. Should drivers be told what they can (or must) do, or what they cannot do. The difference is illustrated in turn restriction messages. "No Right Turn" can be indicated by a right arrow and a red ring with a diagonal slash or with left and straight arrows and a green ring. The latter, permissive, version was (and to some extent still is) used in Canada, while the prohibitive version of such messages is widely used in most parts of the world. Work by Dewar 
and Swanson suggested the merits of the permissive symbol for turn restrictions, but their results could have been a function of the fact that they used Canadian drivers, who were more familiar with the permissive version at the time. ${ }^{(21)}$ However, other research indicated that the prohibitive version of "No Left Turn" resulted in a number of reversals (left turn allowed) and answers such as "road turns left under a bridge ahead." ${ }^{(22,23)}$ In addition, there is evidence that the slash on the prohibitive sign obscures the message. ${ }^{(24)}$ In spite of these findings, at this stage in the development of traffic sign symbols it would appear that the prohibitive restriction symbol is best, in view of its widespread use throughout the world. Uniformity at the international level should be attained to the greatest extent possible.

\section{HIGHWAY SIGN EFFECTIVENESS}

There is a substantial and growing literature on traffic signs that includes a great many of the human factors aspects of sign effectiveness. It is not the intent here to summarize all of this, but to concentrate on those studies that have dealt with traffic sign symbols, with driver age, and with related factors such as conspicuity, legibility distance, glance legibility, reaction time, and symbol comprehension.

\section{Criteria For Traffic Sign Design}

Traffic control devices provide road users with information they may need but would not otherwise obtain. Some sign information is redundant with that provided by other sources (e.g., by observing the presence of a steep hill or an intersection ahead) A significant component of the information is, however, available primarily or exclusively on signs (e.g., changes in speed limit, direction to a destination). Traffic control information is intended mainly for the "stranger," who knows little or nothing about the area traveled, and the "local stranger," who may be generally familiar with the area but does not know the exact location of a destination or presence of a roadway hazard. Such a driver might be traveling in a new part of a familiar city, and know major landmarks, cardinal directions, and major arterials, but not the local residential streets.

Several criteria must be met for a sign to be effective:

- It must command attention or be easily detected by the person who needs the information (conspicuity).

- It must be legible at the appropriate distance so that the driver has time to take the necessary action (legibility distance).

- It must often be legible when seen for a very brief time (glance legibility). At busy urban locations signs can easily be hidden by objects such as large vehicles or other signs and seen only briefly.

- $\quad$ To be effective, a sign must be easily understood (comprehension) or the user will not know how to respond to it.

- It must be understood rapidly, as drivers often have only a second or two to interpret and respond to sign messages (reaction time). 
- Symbol messages must be readily understood or at least easily learned and remembered (learnability). This criterion is often overlooked when designing new symbols and is almost never considered in the evaluation of symbols.

- The information on the sign must be easily rejected if it is irrelevant for the driver.

- The action to be taken in response to the message must be immediately obvious.

- Symbol messages must also fit within a system of signs already in use and relate meaningfully with other systems. That is, they should be distinguishable from other symbols in the system and be consistent in design from one locale to another.

Drivers are often in situations where there is a great deal of potential information in the roadway environment (not only signs). It is not possible to fully attend to and process all this information. The driver must be able to glance at the sign and determine rapidly whether the information is relevant and should be retained and acted upon. Depending on driver needs and driving conditions, all signs are possible sources of relevant information, so the driver must first take in and process the information at a superficial level before it can be decided whether it is relevant. This takes time and a level of mental effort that may not be available to the driver under conditions of high information load or stress. The appropriate action to be taken should not require a significant amount of thinking and decision time, especially if the action must be taken quickly (e.g., yield to oncoming traffic or turn within a very short distance onto a cross street).

The relative importance of these various design criteria has never been established. They are not all of equal importance, and can even be in conflict with one another. In an attempt to determine the relative weighting that ought to be attributed to the main criteria for traffic sign symbols, Dewar solicited the views of sign experts in four countries-Australia, Canada, New Zealand and the United States. ${ }^{(25)}$ Although there were some national differences (e.g., the Australians placed more stress on conspicuity, possibly because there had been a great deal of research on it in that country), there was widespread agreement that comprehension was most important, followed by conspicuity, reaction time and legibility distance (the last two being similar in importance). Learnability was rated lowest. These various criteria were never all measured in one study, nor has there been a systematic effort to assign relative weights to them when more than a single measure was used in conducting an overall assessment.

\section{Factors Determining Highway Sign Effectiveness}

A good deal of research has been done on traffic sign design and effectiveness. For a general review of the road user, sign design and environmental factors influencing the effectiveness of traffic signs, see reference 26. The major sign design factors are examined in this section.

\section{Conspicuity}

No matter how adequate a traffic control device is, it will be of little value if the driver fails to detect it or detects it too late. The major factors that determine the conspicuity of a traffic sign are its size, eccentricity from the observer's direct line of sight, and its color and brightness 
relative to the color and brightness of other objects in the visual environment. Virtually all countries and most smaller jurisdictions have traffic control device manuals that specify with some precision the appropriate placement of signs (e.g., distance from a hazard, height above the road surface). Nevertheless problems associated with poor placement are among the major reasons for failure to acquire sign information. Much of the research on conspicuity of traffic control devices has been done in Australia. ${ }^{(27,28)}$

The environment in which signs are located can greatly affect their conspicuity, as demonstrated by Cole and Hughes, who tested drivers under two conditions. ${ }^{(28)}$ Drivers operated a car in three types of environments: residential streets, arterial roads, and shopping areas, and under two sets of instructions. In one case, drivers were told to report what attracted their attention, while in the other condition, they were to search for, and report target discs, which varied in size and reflectance, located in prominent places along the roadway where traffic signs are typically found. The basis for the detection of discs was "attention conspicuity" (they caught the driver's attention) in the first situation, and "search conspicuity" (they were detected on the basis of a deliberate search) in the second.

Discs in a shopping area were more difficult to detect, under both instruction sets, when in a shopping area due to both visual clutter and driver information load. They were easiest to detect in residential areas. Although target reflectance and size were not strong determinants of conspicuity, the angle of the discs from the drivers' line of sight was. Most target discs were noticed at eccentricities of less than 10 degrees. Their data show that even when a driver is searching for pre-specified targets, not all are detected.

Loo has demonstrated the difficulty of detecting signs embedded in other visual stimu1i. ${ }^{(29)}$ Such situations cannot be avoided, as driver information needs tend to be high where other mental demands are also high (e.g., at intersections).

Mace has reviewed the literature on traffic sign conspicuity and identifies the main determinants of conspicuity to be sign size and luminance, complexity of the visual scene, contrast between a sign and its background, and driver alertness. ${ }^{(2)}$

Conspicuity of the signs among older drivers was determined by Khavanin and Schwab who had drivers indicate as soon as they could see the signs. ${ }^{(30)}$ Neither visual acuity nor contrast sensitivity was highly correlated with detection distance. Detection distances were greater for the 24 -in $(609.6 \mathrm{~mm})$ signs than for the 18 -in $(457.2 \mathrm{~mm})$ ones, and greater for the high intensity material.

It is clear from this and related research that traffic sign information can easily be missed by drivers, in spite of the fact that signs are well designed and placed in accordance with the relevant standards. The remedy to this problem would appear to lie in modifying placement warrants, changing sign design (e.g., size and color), educating drivers to divide their attention more effectively or providing redundancy of sign information. 


\section{Comprehension Of Traffic Sign Symbols}

Highway sign symbology was the subject of an international conference in Washington, DC, in 1972. On the basis of data presented at that meeting, by delegates from around the world, it was evident that comprehension of symbols was relatively poor.

In the early 1960's British road signs were changed from primarily a combination of words and symbols to an almost entirely symbol system similar to that in use then in many European countries. In one of the first evaluations of some of the new symbols Mackie carried out a national survey of over 2000 people. ${ }^{(23)}$ He found that levels of understanding were not high and, for some signs, it was extremely low. The levels of understanding were approximately 50 percent for motorists and 33 percent for non-motorists. Understanding was poorer for the oldest subjects (ages 45 to 64). However, it should be noted that only seven symbols were studied.

In a related set of surveys, Mackie examined understanding of 12 symbol signs, as well as a number of pavement markings in 1965 and again in $1966 .{ }^{(31)}$ Performance was substantially better in the second survey, mainly due to fewer "don't know" responses. Subjects in the oldest group (over 60) had the poorest level of understanding (25 percent), while knowledge was best (48 percent) for the 21- to 25-year-olds.

A large study done in Britain found a great variation in the level of understanding of new symbols that had been introduced on the roads there in the mid-1960's. ${ }^{(32)}$ The percentage of subjects giving correct or "partially correct" answers ranged from 4 to 100 . One particularly disconcerting finding in this study was the frequency of responses that were opposite to the intended meaning and which constituted a potential safety problem (e.g., "overtaking allowed" for "no overtaking "; "route for cars and motorcycles" for "all motor vehicles prohibited") Subjects who drove greater distances performed slightly better than those who drove less. A relatively small proportion ( 31 percent) of subjects learned the meanings of these symbols from official sources such as an "official booklet/poster," while more than half guessed the meaning or "just worked it out."

Research done in Texas on understanding of traffic control devices used a questionnaire with 27 traffic situations involving traffic control devices. ${ }^{(33)}$ The following symbols were seriously misunderstood: "Slippery When Wet," "Pavement Width Transition," the double turn sign, and the curve vs. turn symbols. The effect of driver age was not considered in this study.

The extent of misunderstanding of symbols whose meaning is apparently clear is illustrated in a South African study which reported that, among a certain segment of drivers sampled, 40 percent correctly identified the "Hill" symbol with the response "steep descent," but when questioned were unaware of the meaning of the words "steep" or "descent." ${ }^{34)}$ They had obviously memorized the correct response without understanding the message intended.

In a similar vein, Lewis and Cook reported very different responses when subjects were asked the meaning of, and the action they would take if they saw, the "Watch For Fallen Rock" symbol. ${ }^{(35)}$ About half replied that they would look up to see if rocks were falling and speed up 
to get out of the area; the other half indicated that they would slow down and look down on the road to locate the rocks-two opposite sets of responses to the same symbol. In general, however, if drivers understand a symbol, they know the appropriate action to take. In a group of Canadian drivers, Dewar and Ells found a high level of agreement between meaning and action to be taken when they were presented with a sample of 70 symbols from various countries. ${ }^{\text {(36) }}$

Paniati examined comprehension of a set of 22 symbol warning signs, scoring responses as correct, substantially correct ("not exact, but did indicate a reasonable understanding of the sign meaning"), or incorrect. ${ }^{(37)}$ Compared with other research done in the United States, the level of understanding was very high, with an average of only 5 percent incorrect. There were substantial problems for "Pavement Ends," "Added Lane," "Hill," and "Worker," and there was confusion between "School Crossing" and "Pedestrian Crossing." The rank correlation between the measures of comprehension and legibility distance was .581 ( $\mathrm{p}<.01)$ According to the author, this raises the possibility that comprehension has an effect on legibility distance.

Paniati also studied traffic sign symbols for work zones using several alternatives of each message in an effort to come up with improved designs. ${ }^{(38)}$ Legibility distance, comprehension and preference data were gathered for four messages: "Pavement Width Transition," "Flagger Ahead," "Low Shoulder," and "Uneven Pavement." Previous research had shown poor comprehension of these symbol signs. Current and alternative versions (mostly based on previous work or signs in use in specific locations in the United States) were tested. The percent incorrect in the comprehension test varied from 6 to 59. The overall results were variable in terms of whether the new versions were better than the existing ones. Although the author did not attempt to combine the three measures in any way, there appeared to be little consistency across these measures for individual messages. Age differences were not examined in this study.

One of the most extensive studies of drivers' understanding of traffic sign symbols was undertaken for the FHWA by Knoblauch and Pietrucha, who examined potential deficiencies in approximately $30 \mathrm{U}$. S. symbols and made recommendations for their improvement. ${ }^{(39)}$ On the basis of existing research on symbol signs they identified deficiencies in understanding which might pose safety or operational problems. The study began by gathering input from a variety of professionals: traffic engineers, driver educators, safety specialists. These experts were asked to comment on any signs that they felt presented "problems" with comprehension, conspicuity, legibility, compliance, etc. The signs identified as problem signs by these experts were then examined in terms of the type of miscomprehension, degree of miscomprehension, and the consequences of miscomprehension. Each sign was given a rating based on a combination of these factors and those rated worst were further studied in a laboratory experiment. Alternate designs of a number of messages were then developed and tested in a driving simulator. Certain "families" of signs were found to be quite confusing (e.g., curves vs. turns; pedestrian vs. school signs).

Efforts to redesign the messages for better understanding met with limited success. A close examination of their data shows that, on average, fewer than half of the alternate versions of the 30 symbols tested were an improvement over the originals (from the MUTCD). Many were substantially worse in terms of comprehension. This illustrates the difficulty of designing and redesigning pictographic representations of traffic messages. This study, as with most work 
testing completely novel symbols, had the problem that the new and the redesigned versions were novel to subjects, therefore less likely to be understood due to their unfamiliarity.

In a study of driver understanding of work zone signing, Ogden, et al. evaluated three symbol signs using a multiple choice questionnaire. Drivers understood the "Advance Flagger," "Low Shoulder," "Lane Ends" symbols 77.5 percent, less than 16 percent, and 78.4 percent of the time, respectively, when the signs were shown alone. ${ }^{(40)}$ When shown in the context of a roadway scene (with a photograph), the levels of understanding improved slightly.

Hawkins, et al. surveyed 1745 Texas drivers to determine their level of understanding of 17 warning signs, 10 of which were symbols. ${ }^{(41)}$ Level of correct comprehension varied from about 32 percent (“Turn") to 87 percent (“Stop Ahead”), with an average of 61 percent for the symbol signs. For the text signs the average level of comprehension was also about 61 percent, with a range of 29 percent to 89 percent.

A sample of 244 drivers in Wisconsin were surveyed about their knowledge of 12 traffic sign symbols. ${ }^{(42)}$ The overall "failure rate" was 15.5 percent, with the percentage of correct responses ranging from 68 percent ("School Zone") to 98 percent ("No U-Turn"). Among the most poorly understood symbols were "Right Lane Ends" and "Divided Highway Ends." This overall correct response rate was an improvement over that in a larger survey a few years earlier, when the failure rate was 29 percent. The research on traffic sign symbol comprehension illustrates the importance of designing "user-friendly" symbols that are easily understood by naive drivers.

\section{Legibility Distance}

It is essential that traffic sign information be received and processed by the driver at a sufficient distance (i.e., in sufficient time) to take whatever action is required. Several factors determine legibility distance: sign size and brightness, background luminance, contrast between the sign legend and its background, visual complexity of symbols, length of word messages, the visual abilities of the driver, and environmental factors such as darkness, fog, or rain. Next to comprehension, legibility distance is probably the most widely used criterion for symbol evaluation.

Khavanin and Schwab evaluated the legibility and conspicuity of text traffic signs under day and night driving conditions with a sample of 48 older drivers, who were required to read aloud and press a button when they could read the signs while driving toward them. ${ }^{(30)}$ As expected, legibility distance increased with increases in letter size: $10.16,15.24$, and $20.32 \mathrm{~cm}$ $(4,6$, and 8 in), while there was a slight, but not statistically significant, difference between engineering and high intensity grade sign material under the night condition. The authors point out that, while legibility distance increased with letter size, there is a point of diminishing returns and that the relationship between distance and letter size did not come close to the $6 \mathrm{~m} / \mathrm{cm}$ (50 ft/in) legibility index often used to decide on letter height for specific roadway applications.

In an experiment on traffic signs carried out in Canada, subjects drove an automobile toward standard traffic signs on a two-lane rural highway under conditions of light traffic during the daytime. ${ }^{(43)}$ Speeds of either 48 or $80 \mathrm{~km} / \mathrm{h}$ (30 or $\left.50 \mathrm{mi} / \mathrm{h}\right)$ were maintained while subjects 
drove one at a time toward the signs. They were required to identify the sign as far away as they could-a measure of legibility distance. The distance at which the sign was correctly identified was measured to the nearest foot by instrumentation in the vehicle. The stimuli were eight symbol and eight word signs, half of them warning signs and the other half regulatory messages. The symbol signs could be identified, on average, at a distance of $24.69 \mathrm{~m}(861 \mathrm{ft})$, approximately 59 percent farther than the word signs.

In a scaled-down version of that same roadway procedure, with the same signs at $1 / 3$ the dimensions of standard signs and subjects driving at approximately $27 \mathrm{~km} / \mathrm{h}$ or $17 \mathrm{mi} / \mathrm{h}(1 / 3$ of 50 $\mathrm{mi} / \mathrm{h}$ ), the symbol signs had an 84 percent advantage in legibility distance over the word signs. Differences in favor of symbol signs are to be expected, in view of the larger and simpler visual components in most symbols. The age variable was not examined in either of these studies.

An experiment done in Australia examined symbol and alphabetic traffic signs to determine their legibility distances under various degrees of blurred vision. ${ }^{(18)}$ The 50 percent threshold legibility distance for symbol signs was about double that for the word versions of the same 16 messages. The thresholds, as measured with their laboratory method, were $270 \mathrm{~m}$ $(885.83 \mathrm{ft})$ for the symbol signs and $142 \mathrm{~m}(465.66 \mathrm{ft})$ for the word signs under conditions of unaltered visual acuity (average acuity of subjects was 6/4.3). As visual acuity was reduced through optical blurring, the decrease in legibility distances was proportionally greater for the word signs. The effects of observer age were not addressed.

\section{Glance Legibility}

As indicated earlier, it is important under certain driving conditions to be able to recognize traffic signs that are seen for a very brief intervals, under certain driving conditions. Several studies have examined glance legibility of signs. Dewar and Swanson studied the ability of subjects to recognize briefly presented signs of three different types: symbol, word and symbol/word combinations (i.e., the symbol message was repeated in words). ${ }^{(21)}$ The stimuli, all turn restriction messages, also included some signs with time designations on them (e.g., "No Turns 7 to 9 am"). In addition, they tested both permissive (turn arrow inside a green ring) and prohibitive (turn arrow inside a red ring with red slash) symbol versions of some messages. The stimuli were slides of traffic signs either in their natural environment, hanging above an intersection of two urban streets, or simply as large pictures of the signs themselves, not in context. The former stimuli were presented by projection tachistoscope to groups of drivers for $200 \mathrm{~ms}$, while the exposure duration for the signs alone was $40 \mathrm{~ms}$.

Results indicated that the permissive turn restriction symbols were more readily identified than either the prohibitive symbols or words alone, and the positive symbols had greater glance legibility than words alone. The only message in which the words were more readily identified than the symbol was the "No U turn" message. In general, symbol signs, when compared with others containing the additional word information and or time, were found to be more easily identified. Although no effort was made to test subjects across a wide age range (very few were over 50), the younger subjects performed better on four signs. However, there was no specific type of sign on which they were better than older subjects. 
In a study comparing symbol and word messages Avant, Brewer, and Thieman, using a tachistoscope to measure glance legibility, found that fewer errors were made in identifying the symbol versions. ${ }^{(44)}$ However, exposure durations were very brief $(32,41$, and $49 \mathrm{~ms})$ and subjects were mainly young or middle-aged drivers.

\section{$\underline{\text { Reaction Time }}$}

Reaction time, or the latency with which drivers can detect and respond to a traffic sign, was not often measured in sign evaluation research until the 1970's. This measure was one of a number of criteria evaluated in a series of experiments by Dewar and his colleagues. They examined the same set of traffic signs--half symbol and half word, split evenly between warning and regulatory signs. Two different sizes of signs were used, corresponding to the sizes of images on the retina that would be made by a standard traffic sign viewed at the stopping distance required under normal driving conditions for a car traveling at 48 and $80 \mathrm{~km} / \mathrm{h}$ (30 and $50 \mathrm{mi} / \mathrm{h}$ ). In one experiment, the response used was the verbalization of the name of the sign.(36) Results showed a slight advantage to the word signs, which were identified about 9 percent faster than symbol messages.

Further research by Dewar, Ells, and Mundy employed visual distracter tasks. In the first of these, subjects were required to respond, as in the previous experiment, to the signs by verbalizing their meanings, while at the same time monitoring a series of numbers presented which appeared above the image of the traffic signs. ${ }^{(45)}$ Subjects were to depress a button when specific target stimuli appeared in the number task. The addition of a loading task elevated the reaction times, but did not otherwise alter the influence of the other variables nor the interactions that occurred in the first experiment. Verbal signs were responded to about 18 percent quicker than were symbol signs.

The third experiment in the series, using the same signs, employed a different visual loading task-monitoring, and correcting as necessary, the speed level on a speedometer. The speed changed an average of once every $20 \mathrm{~s}$. In addition, visual distraction was introduced by projecting the slide of the sign onto a color movie film of a straight roadway, taken through the front window of a vehicle traveling at $80 \mathrm{~km} / \mathrm{h}(50 \mathrm{mi} / \mathrm{h})$. Results indicated no differences in reaction times to symbol and verbal signs.

It was apparent that in these first three experiments the direction of the difference, when it occurred, between symbol and word signs (word signs faster) may well be due to an artifact, namely, that the stimulus and the response were of greater compatibility (both being words) for the word signs than for the symbol signs. To avoid this confound, a different response was used in further work. ${ }^{(20)}$ It required subjects to make the verbal response "yes" if the sign shown on a screen was the same as a message presented vocally by the experimenter just prior to the stimulus presentation, and otherwise to say "no." The subject's responses activated a voice key. Symbols were responded to more quickly than words, thus this "yes-no" procedure eliminated the advantage to word messages, suggesting that the faster responses to word signs in the earlier experiments were an artifact based on level of stimulus/response compatibility.

An important but seldom addressed issue in research on sign effectiveness is the measurement of validity. That is, are signs which are most effective as indicated by laboratory 
measures of performance the same ones which perform best on the road? Ells and Dewar examined this issue by correlating reaction time with the roadway legibility distance measures of the same signs. ${ }^{(2)}$ The correlations were consistently high for the word signs, but less so for the symbol signs, possibly due to the high stimulus-response compatibility referred to above.

In order to demonstrate that this reaction time measure using the "yes-no" procedure correlated with the on-the-road measure of legibility distance, as had the reaction time index in the previous experiments, the same set of 16 signs previously tested in the roadway study were examined. ${ }^{(20)}$ The mean reaction time to the word signs was $690 \mathrm{~ms}$, which was 13 percent greater than to the symbol messages. The correlations between roadway legibility distances and reaction times to these signs were: -0.62 for "yes" responses and -0.75 for "no" responses. Both correlations were statistically significant ( $<$ <.01), indicating that the "yes-no" procedure was a valid index of traffic sign symbol perception on the roadway.

Having demonstrated that the "yes-no" response procedure was an appropriate approach, the authors used this method to determine reaction times to both the symbol and word versions of a set of seven warning and seven regulatory messages. Subjects were tested under normal -viewing conditions and under the influence of visual degradation created by veiling glare that produced a 50 percent reduction in visual acuity. Reaction times were about 17 percent faster for symbol messages than for their word counterparts. Both types of signs were responded to more slowly under the degraded condition, but this deterioration was much greater for the word versions, illustrating the advantage of symbols under less than ideal visual conditions.

Whitaker studied driver response times to words, symbols (arrows) and the combination of these for directional messages, for example, "Do Not Turn Right," Left Arrow (for Turn Left)

Results showed faster response times to symbols than to words or to symbols plus words. ${ }^{(46)}$ The redundant information did not influence reaction time. Responses to negative messages ("Do Not Turn Left") were slower than to positive ones ("Do Turn Right"). The participants were all young adults, however, so the study tells us nothing about possible interactions between age, sign type, and message.

\section{Combinations of Measures}

As indicated earlier, research on traffic signs typically involves a single measure, or at most two. It is evident that several variables determine the effectiveness of a sign, so it is appropriate to include at least the most important ones when evaluating signs.

One of the few studies to use and combine a number of measures was that of Roberts, Lareau, and Welch, who compared the symbol and text versions of 19 traffic sign messages. ${ }^{(47)}$ Most messages had one text and four symbol versions. Five measures were used: understanding time (the time required to indicate a sign's meaning), comprehension, certainty (how confident the subject was of understanding the sign's meaning), preference (rank ordering of the symbols used to convey a specific message) and identification time (minimum exposure time at which subjects could accurately identify all elements of the symbol).

The authors derived an "efficiency index" for each symbol version of each messagewhat they called the "relative 'goodness' of performance" of that symbol. For each individual 
symbol, the Z-score for each variable was calculated "based on the position of its mean relative to the overall distribution of means for that particular variable across all signs." $(\mathrm{p}<.10)^{(47)}$ The efficiency index for a specific symbol was the sum of the Z-scores for the five variables. It was assumed that each variable contributes equally to the overall index, a widespread assumption that has apparently never been properly examined by traffic sign experts. Only one meaningful correlation was found among the five measures ( +.28 between understanding time and certainty of response), suggesting that these variables are measuring different factors in symbol effectiveness.

Mackett-Stout and Dewar, in a study of public information symbols, modified the method of calculating the Z-scores for the efficiency index, basing it on the data from each message, rather than on means for the particular variable across all messages. ${ }^{(48)}$ They argue that the Roberts, et al. procedure could result in a low value of the index, even though a particular version of a message was the best of those tested. The adequacy of a symbol ought to be measured relative to other symbols representing that same message, otherwise the measure of performance is dependent on the context (the total set of symbols being tested). The approach used by Mackett-Stout and Dewar allows a more meaningful index of the adequacy of each symbol version of a message as compared to the other versions of that message.

The use of an index of this type allows the combining of a number of measures of symbol effectiveness. However, decisions still need to be made on the basis of the absolute scores on these measures. For example, it would not be wise to adopt a symbol version of a message simply because it was the best of four tested, if the level of comprehension of all versions was low. It is clear that more systematic attention needs to be paid to the relative weights to be put on the various measures used in symbol evaluation.

\section{Environmental Determinants Of Sign Effectiveness}

\section{$\underline{\text { Illumination Level And Glare }}$}

A host of environmental/roadway factors can influence the effectiveness of a sign. One of the most obvious is low levels of illumination. Sign manuals specify that signs must be manufactured so as to give the same appearance night and day. For example, the 1988 version of the United States Manual of Uniform Traffic Control Devices (MUTCD) specifies that, "All signs intended to be used during the hours of darkness shall be either reflectorized with a material that has a smooth, sealed outer surface, or illuminated to show approximately the same shape and color, day and night." Technology has led to the use of high intensity materials which reflect high proportions of the light falling on them. Retroreflective sheeting has tiny glass beads that act like mirrors to reflect the light back in the direction from which it came. For this reason, it is essential that signs be placed correctly and that vehicle headlights be aimed so as to maximize their benefits. Some vehicle lamp manufacturers have attempted to minimize glare to oncoming drivers by having low and sharp cutoffs for the head lamp beam, but this can result in insufficient light falling on overhead signs. The major sign design factors influencing nighttime legibility have been examined by Olson and Bernstein. ${ }^{(49)}$

\section{Weather Conditions}


Another environmental factor which is obviously detrimental to sign perception involves atmospheric conditions that reduce visibility (e.g., rain, snow, fog). The potential effects of the combination of fog and darkness were demonstrated by Ells and Dewar, who used a laboratory simulation to create veiling glare of the sort that would be expected while driving toward oncoming headlights at night in the fog. ${ }^{(20)}$ As reported earlier, this condition produced longer times to identify signs as compared to no veiling glare, and the detrimental effects were considerably worse for word signs than for symbols.

An environmental factor often ignored by those concerned with sign maintenance is dirt on signs. Rumar and Ost report that, under unfavorable conditions, dirt accumulation can reduce reflected light and contrast on small traffic signs up to 75 percent and 95 percent respectively. ${ }^{(50)}$ This is likely to present a special problem with signs at construction sites, where the accident rates are higher than at other roadway locations.

\section{$\underline{\text { Windshield Conditions }}$}

A significant problem with visibility is created when the windshield transparency is reduced due to mud, snow, rain, dust, bugs, etc., on the outside, and smoke particles, condensation on the inside. Dirt or film on windshields not only reduces the amount of light getting to the driver's eye, but also poses an important source of veiling glare, as the light is scattered and forms a film of light, thus reducing the contrast of objects in the roadway environment. Scratches on the glass can also contribute to glare problems. Allen estimated that windshields should be replaced every $80000 \mathrm{~km}(50,000 \mathrm{mi})$ to maintain optimum vision at night against headlight glare. ${ }^{(51)}$ The windshield can also create glare by reflecting bright objects, such as light dash boards, inside the vehicle.

Another factor determining visibility is the degree of tinting of the windshield. This is done to reduce brightness of sunlight, and to minimize heat buildup on hot sunny days. As compared with clear glass, however, tinted windshields reduce the transmission of light, especially red light.

\section{SYMBOL SIGN DESIGN AND EVALUATION}

There is a great variety of methods for evaluating signs. There appears, however, to be little agreement on either the major criteria that should be tested, or on the most appropriate procedures for measuring these criteria. Methods can be broadly categorized as either field (onthe-road), or laboratory measures, as outlined by Dewar and Berger. ${ }^{(52)}$ Since their analysis of methodologies for sign evaluation over 2 decades ago, a number of more sophisticated approaches have come into use, particularly those using computers and driving simulators. This brief review of methodology will first examine traditional approaches, then those developing technologies that will likely be used more frequently in future research.

\section{Traditional Methods}

The design of traffic signs has traditionally been the work of traffic engineers. Only in the past 2 or 3 decades have behavioral scientists and human factors specialists also played a part in their design and evaluation. One of the most commonly used approaches to symbol sign 
design has been to create a committee composed primarily of traffic engineers, or use the services of existing ones (e.g., the National Committee on Uniform Traffic Control Devices), as done in the United States, which examines alternate symbol versions of a message and recommends sign designs to be used. The decision have often been based largely on subjective input of committee members. Consequently, the design of symbols in places such as Australia, Canada, and the United States, has for many decades been based primarily on non-scientific information from traffic engineers and other "traffic experts" (e.g., police, planners). More recently, however, the need for scientific data to support the development of a symbol has been recognized, and human factors experts are now more often involved in the relevant research. Even then the procedures for symbol design are not consistent, and evaluation research typically involves a single measure--comprehension. ${ }^{(53)}$ The need to make use of a number of measures, and if possible, to combine them in some meaningful way, has been discussed earlier. ${ }^{(48)}$

Symbol versions of traffic signs which become candidates for evaluation in the development of a symbol are often taken from existing systems, used on a trial basis by one or more jurisdictions (e.g., a city or State), or are the product of the imagination of an engineer or graphics artist. Seldom is proper consideration given to the task requirements of driving and the information processing capabilities of drivers, especially the older driver. The lack of scientific input to symbol design and the need for more research on this topic have been outlined by Shapiro, et a1. ${ }^{(54)}$

A useful approach to the development of symbols is that used by the International Organization For Standardization (ISO) Committee on Public Information Symbo1s. ${ }^{(55)}$ Once the need for a symbol to represent a specific message has been determined, the first step is to gather a number of candidate symbols that might be appropriate. This can be done by reviewing signing practices elsewhere to determine whether some existing symbols (if any) merit consideration. Additional ideas come from using the "production" method, whereby a number of relevant experts and users (drivers) generate possible designs for the intended message. These two approaches will typically provide several candidate symbols for further evaluation. However, it is generally necessary to reduce their numbers to three or four substantially different versions (e.g., a bus viewed from the side or from the front, with several windows, or only a few) for further testing. The selection of a few versions from among many is a matter of judgment, ensuring that no two versions are very similar, and that none is likely to be incomprehensible, or contains a great deal of small detail. This is often done by having sign experts rate the appropriateness of each version.

The next step is to conduct a series of studies to determine how well each version meets the major criteria for a good symbol. The practice of the ISO has been to evaluate comprehension using a sample of people of different ages from around the world (usually 6 to 8 countries) The data are then analyzed and decisions made concerning which version is best and whether this one meets minimum standards of understandability (often taken as $2 / 3$ correct).

Unfortunately, the evaluation of symbols often stops at this point with no effort to test any of the other important criteria such as legibility distance, glance legibility and recognition time. The need for these additional measures and some of the procedures for implementing them are outlined by Dewar and El1s. ${ }^{(53)}$ In the past, sign stimuli have often been evaluated using color slides, tachistoscopically presented in the case of glance legibility, to which drivers make a 
verbal or manual (e.g., button press) response indicating recognition. A variety of methods are also available for determining levels of comprehension: write the meaning of a symbol on an answer sheet, select the most appropriate answer in a multiple choice format, or rate the clarity of a symbol's meaning. Having subjects write out the meanings of symbols is the most time consuming method, but it provides the richest data, allowing determination of the nature of the errors and confusion among symbols within the same signing system.

Signs may be shown in isolation, in an artist's rendition of the roadway context in which they normally appear, or in a photograph, film, or video of the actual roadway environment in which they appear. ${ }^{(39,56)}$ The presence of context appears to aid drivers in getting the correct response. Reaction time measures generally present the stimulus in the form of a slide and have subjects indicate its meaning as quickly as possible. ${ }^{(36)}$

Legibility distance measures may have subject move toward the sign or the sign toward the subject, to determine the distance at which the sign message is legible. ${ }^{(36,48)}$ Other approaches involve increasing the size of the image on a screen until the subject can describe its contents or identify the message. ${ }^{(57)}$ Glance legibility procedures present the sign for a very brief duration (e.g., $30 \mathrm{~ms}$ to several hundred $\mathrm{ms}$, depending on stimulus complexity) to determine what proportion of the time the message can be identified, or to increase the stimulus duration until the message is identified. ${ }^{(24)}$

\section{Emerging Technologies}

The use of computers and driving simulators has seen the application of more sophisticated techniques for sign evaluation. ${ }^{(39,58)}$ These devices make the study of several sign criteria more efficient than in past decades, and permit the introduction of additional variables such as subsidiary loading tasks, vehicle handling characteristics, environmental conditions, etc. However, this is somewhat more expensive than other methods and has the problem of simulator sickness, especially with older subjects.

Another method that has shown success in designing more effective symbol signs is the low-pass optical technique of Kline and Fuchs, who were able to increase the legibility distances of symbol highway signs for young, middle-aged and older drivers. ${ }^{(57)}$ To identify and thus avoid the problems of contour legibility and interaction between. adjacent contours in regulation symbol highway signs, the experimenters viewed versions of them blurred by strong positive sphere lenses. The resulting legibility distances and comprehension of standard text, standard symbol, and the "improved" symbol highway signs were then compared among young, middleaged, and older observers. The average distance at which standard symbol signs could be identified was about two times that of standard text signs. The legibility distances of their improved symbol signs, however, were about three times those of standard text signs, and 50 percent greater than those of standard symbol signs, demonstrating that their optical approach can be used to enhance the legibility of symbol highway signs for drivers of any age.

Although only in the initial stage of its application to the design of highway signs, an image-processing linear systems approach may offer an even more powerful design tool than the low-pass optical approach for optimizing the legibility of highway signs. ${ }^{(59,60)}$ The linear systems approach is based on the premise that an image can be equivalently represented in both a 
spatial and a frequency domain making a linear (i.e., a Fourier) transformation between the two domains possible. ${ }^{(61)}$ Using this approach, it is possible to quantify a complex 2-dimensional image, such as a symbol sign, in terms of its Fourier components (spatial frequency, orientation, amplitude and phase angle), and to specify the particular Fourier characteristics that produce the most legible signs. In addition, forward and inverse transform procedures, in conjunction with contrast sensitivity procedures, allow the designer to display a sign on-screen as it might be seen by a particular design observer. ${ }^{(62)}$

The forward transform of a digitally-represented image involves modifying its digitally-represented image using some particular weighting or modulation transfer function (MTF). ${ }^{(63)}$ The resulting product image can then be inverse transformed and displayed on-screen. It has been shown recently that the contrast sensitivity functions (CSF's) of drivers of different ages can be used as weighting functions to yield age-group-specific predictions of the legibility of different symbol highway signs. ${ }^{(59,60)}$ These investigators reasoned that among young and middle-aged observers the legibility distances of symbol signs filtered using an older observer's CSF should be similar to those of older observers viewing unfiltered versions of the same signs, and that the legibility of images filtered through the observer's own CSF should not be different than if the sign's image was not filtered at all. To evaluate these hypotheses, the CSF's of young, middle-aged and older observers were determined along the three principal orientations (vertical, horizontal, and diagonal) Legibility distances were then determined for symbol signs classified in terms of their Fourier power spectrum as low, medium, and high-spatial frequency, each filtered in one of three different ways: not at all (Unfiltered), through the observer's own CSF (Self-filtered) and through the median CSF of another age group (Other-filtered). For young and middle-aged observers, the Other-filtered condition was based on the median CSF of the older group, while for older observers, the Other-filter condition was based on the median CSF of the young group. As predicted, the legibility distances of the Unfiltered and Self-Filtered versions of the signs were not different in any of the three age groups. Among young and middle-aged observers, legibility distances in the Other-Filtered condition were reduced dramatically compared with the Unfiltered and Self-Filtered conditions. Further, the reduction in legibility was greatest for high spatial frequency signs, and least for low spatial frequency signs. No such differences, however, occurred among older observers; legibility distances were almost identical in the Unfiltered, Self-Filtered, and Other-Filtered conditions. These findings demonstrate that image-processing techniques can be employed to "look through the eves of other observers" and to optimize the legibility of complex spatial displays such as symbol traffic signs.

\section{Standards For Symbol Effectiveness}

The matter of establishing when a symbol is well understood is not an easy one. Not only should a broad national sample of drivers (and pedestrians) be studied, but the most appropriate methodology for evaluating symbol comprehension must be more clearly established. There are a variety of procedures, not all of equal validity, some of which would be too expensive to be practical in a large-scale study. Assuming that adequate methods and sampling were attainable, there is still the issue of just what proportion of road users must understand a symbol in order for it to be safely used. Criterion levels of 65 percent correct comprehension have been used in some countries, but there is generally no clear statement of what is an acceptable level of understanding, nor to what degree significant departures below this level for any particular group 
of drivers (e.g., recent immigrants, the older driver) is tolerable. It would seem obvious that the consequences of failure to understand any particular symbol should also be taken into account. It is more important to identify and respond correctly to a "Do Not Enter" symbol than one indicating "Accommodation." The relative importance of different messages has apparently not been addressed as it relates to symbol use. There also appears to be no established standard for symbol legibility, response latency, or conspicuity. These concerns should alert traffic sign designers to the fact that not all messages can or need to be symbolized.

As can be seen from this brief account of the methods used for traffic sign evaluation, a great many techniques exist. There is no common agreement on which are the "best" methods for measuring each of the criteria for traffic sign effectiveness.

\section{SENSORY, PERCEPTUAL, COGNITIVE, AND PSYCHOMOTOR AGING}

\section{Age-Related Sensory, Perceptual Cognitive, And Psychomotor Change}

The last 2 decades have seen a dramatic growth in the research literature on most aspects of aging, including the sensory, perceptual, cognitive, and psychomotor changes that accompany old age. Since it is not possible to include all of the work that might bear on age differences in sign effectiveness, the reader is also referred to other reviews on these topics. (See references 2, $64,65,66,67,68,69,70,71,72,73,74,75$.

Age-related changes can have an adverse effect on a driver's ability to perceive and react to a variety of roadway elements, including signs. For this reason, it is essential to design signs for a "design driver" whose characteristics match those of older drivers. Unfortunately, driver age is seldom a consideration in design standards. For example, the visual acuity assumed by the standard for sign letter height in the United States is 6/7 (20/23), a level attained by very few older drivers, even with visual correction.

In a review of the needs and limitations of older drivers, Mace outlines the concept of Minimum Required Legibility Distance- $t$ h e distance from a sign required by drivers to detect, understand, make a decision, and initiate and successfully implement a vehicle maneuver before reaching the sign. ${ }^{(2)}$ Limitations of older drivers which increase this distance include: increased detection time due to distraction by irrelevant input, longer time to understand unclear messages (e.g., some symbols), inability to read signs at great distances and under adverse viewing conditions due to poorer acuity and contrast sensitivity, and longer decision times (this last factor may be the most important in increasing the required distance). In his paper, Mace summarizes the literature on the major variables that contribute to sign legibility and conspicuity--visual complexity of the highway scene, size and luminance of the sign, format of the lettering and symbols on the sign, and driver alertness and visual abilities.

Shinar and Schieber have summarized the visual requirements of older drivers, including static acuity (photopic, mesopic, and in the presence of glare), dynamic acuity, visual field, contrast sensitivity, and motion perception. ${ }^{(76)}$ They point out that the research on vision and driving shows only a weak relationship between these two, and that the studies were correlational, thus not necessarily demonstrating a causal relationship. One finding revealed that 
improper lookout among drivers with poor vision was three times more likely to be found as a causal factor in their accidents than with those without poor vision. ${ }^{(77)}$ Shinar and Schieber report data from 890 healthy drivers with no known visual diseases which show deterioration in simple visual tasks (visual acuity under photopic, mesopic, and glare conditions) with age, especially for those over 65. This deterioration with age (beginning about age 50) was much greater for three complex tasks-dynamic visual acuity, central angular movement threshold, and central movement in depth threshold. These last three visual functions deteriorate at a much faster rate than do the simpler functions.

\section{The Eye}

Ocular Changes. With increasing age there is a decline in pupillary diameter (senile miosis) that is particularly pronounced at low light 1evels. ${ }^{(78}$ ) Although this change causes a significant reduction in retinal illumination among older observers, it can also reduce optical aberration and increase the range over which stimuli are in focus (i.e., the depth-of-field). Light absorption by the senescent lens is elevated, especially for short wavelength light (i.e., blue). ${ }^{(79)}$ Weale has estimated that, to compensate for the reduction in retinal illuminance which is due to pupillary miosis and changes in the optic media, a three-fold increase in illuminance is needed by age $60{ }^{(80)}$ There is also increased intraocular light scatter in the older eye, reducing contrast in the retinal image. ${ }^{(81,82)}$

Accommodation. With age, there is a well-recognized loss in the amplitude of accommodation (i.e., presbyopia), so that past 60 or so virtually no accommodative capacity remains. ${ }^{(83)}$ This problem is exacerbated by low light levels; as illumination falls, observers are biased toward their dark focus. When Simonelli measured the dark focus in observers aged 17 to 76, it declined with increasing age at approximately the same rate as the far point for near vision. ${ }^{\left({ }^{84}\right)}$ Elworth, Larry, and Malmstrom assessed the speed of accommodation in 35 observers aged 20 to 54 years, and found that older observers could accommodate as quickly as younger ones under ideal circumstances. ${ }^{(85)}$ Under degraded conditions, including a reduction in ambient illumination, the accommodation time of older observers was many times greater than that of their younger counterparts.

Color Discrimination. The data regarding age-related losses in color vision are somewhat equivocal. Some studies have found little or no age differences and others have reported a decrease in color sensitivity that is most pronounced for shorter wavelengths. (See references 86 , 87, 89, 90.)

A lo-year longitudinal study by Gittings, Fozard, and Shock found very little change in the color vision of 577 males aged 20 to 95 years. ${ }^{(91)}$ Knoblauch et al., however, found that errors on the Farnsworth-Munsell 100 Hue Test increased with age, primarily along the blue/yellow axis. ${ }^{(92)}$ Errors increased as illumination was reduced, suggesting that lowered retinal illumination might account for some of the deficit. This suggestion is consistent with the results of Boyce and Simons, who found that increasing illuminance from 400 to 1200 lux improved the performance of their old group on the Farnsworth-Munsell test of color vision, but did not benefit young observers. ${ }^{(93)}$ When Eisner, Fleming, Klein, and Mauldin studied color matching to a $588 \mathrm{~nm}$ stimulus in observers 60 and older, they observed a small but significant decrease in sensitivity of about $0.01 \mathrm{log}$ units per decade. ${ }^{(94)}$ They suggested that this difference 
reflected receptor losses in addition to a reduction in retinal illumination. Little is yet known regarding the possible interactions between age differences in color vision, and the spectral characteristics of different illuminating sources that might affect display legibility or visual comfort.

Oculomotor Changes. Dynamic oculomotor performance declines in older adults. Smooth pursuit eye movement is impaired, the extent of upward gaze is more restricted, and the duration and velocity of large saccades are lower. ${ }^{(95,96,97)}$ The ability to maintain stable gaze, however, appears to remain relatively unimpaired in healthy older observers. ${ }^{(98)}$

\section{Sensitivity To Light}

Nighttime driving with head lights, like many common night vision tasks, is characterized by mesopic and low photopic illumination conditions. ${ }^{(99)}$ It has been clearly demonstrated that visual sensitivity declines significantly as stimulus luminance decreases from daytime to low photopic and/or mesopic levels.

Dark Adaptation. The process of adjusting the eye's sensitivity to lower illumination (i.e., dark adaptation) is mediated by both neural and photochemical processes. Studies of age differences in dark adaptation show a marked elevation in the final threshold of both the photopic and scotopic components of the curve. Birren, Bick, and Fox examined age differences in the minimum light threshold of the dark-adapted eye and found an increase of approximately $1.3 \log$ units from the 20's to the 80's.(100) McFarland and Fisher derived dark adaptation curves for observers aged 20 to 60 years and found a correlation of 0.89 between age and final threshold. ${ }^{(101)}$ Subsequently, McFarland, Domey, Warren, and Ward found a high correlation between the age of the observer and thresholds of both rods and cones throughout the adaptation period. ${ }^{(102)}$ They concluded that the rates of adaptation of both the rod and cone systems were inversely related to age.

Weale, however, used the data of Birren and Shock to argue that the rate of dark adaptation is unchanged with age. ${ }^{(103,104)}$ He showed that the slowing in rate of adaptation reflected simply a delay in reaching any particular sensitivity level due to an age-related upward shift of the entire adaptation function. When Eisner et al. studied dark adaptation in the fovea of observers aged 60 to 88 years who possessed excellent acuity, they found an elevation of $0.09 \log$ units per decade, but no age difference in adaptation rate. ${ }^{(94)}$

Weale compared the data from several studies of dark adaptation that varied the wavelength of the test light used, and controlled pupil size. ${ }^{(105)} \mathrm{He}$ had concluded that age differences in visual threshold were due mainly to reductions in retinal illuminance associated with pupillary miosis and to increased lenticular opacity. The remainder of the loss may be attributable to changes in retinal metabolism and to degeneration of the neural pathways. ${ }^{(106)}$

Difference Thresholds. The differential threshold for light expressed as a Weber fraction (DI/I) increases with age, especially past age $60 .^{(107)}$ There is evidence that the change in differential threshold is somewhat wavelength-specific. Prestrude, Levenick, and Woody observed that the increase in the Weber fraction for short wavelengths was most noticeable past age 40, an effect congruent with the absorption of blue light by the senescent crystalline lens. ${ }^{(79,108)}$ 
Glare and Glare Recover-v. Light that is excessively bright or inappropriately directed can act as a glare source and impair visual functioning. This can occur when stray light is distributed across the retina, reducing contrast (veiling glare); if details to be discerned are presented in an overly bright visual display (dazzling glare); or during the extended recovery period after transient exposure of the retina to an intense light source (scotomatic glare). Susceptibility to glare of all three types appears to increase during adult aging. ${ }^{(109)}$ The most common form of disability glare while driving at night is a reduction in visual efficiency caused by "veiling luminance" of stray light superimposed upon the retinal image.

Wolf measured age differences in the luminance required to identify targets with and without veiling glare. ${ }^{(81)}$ Even in the absence of glare, the illumination required to identify targets increased with age. Under glare conditions, age differences were increased, especially past age 45. Although Sturgis and Osgood found that the effects of glare were greater among older persons, the difference was directly proportional to the age-related elevation in luminance thresholds in the absence of glare. ${ }^{(99)}$ They concluded that the glare effect is not increased in older persons, and that a low-luminance acuity test would provide an effective evaluation of visual fitness for night driving.

Pulling, Wolf, Sturgis, Vaillancourt, and Dolliver determined age differences in identification of the gap location of Landolt rings in the presence of glare. $\left.{ }^{(110}\right)$ Resistance to physiological glare and to headlight glare were both found to decrease with age. Such age differences in sensitivity to glare often have been explained by age changes in the ocular media, specifically lenticular fluorescence and scatter.

Available evidence suggests that recovery from glare is protracted in the senescent visual system. Burg evaluated age differences in illumination thresholds for form recognition before and after exposure to a scotopic glare source. ${ }^{(111)}$ Illumination thresholds for form recognition in glare, as well as-recovery time from glare, were markedly greater among older observers. Glare recovery times were $3.9,5.6$ and 6.8 s. for drivers 20 to 24,40 to 44 and 75 to 79 , respectively. Reading found that time to recover from headlight glare increased with age, a recovery period that was more extended after exposure to white than to yellow light. ${ }^{(12)}$ Olson and Sivak, in a study of glare from automobile rear-vision mirrors, also found that older observers were slower to regain their sensitivity. ${ }^{(113)}$

Recent studies of disability glare suggest that contrast sensitivity paradigms may represent a powerful means of assessing age differences in the magnitude of glare effects. ${ }^{(114)}$ The work of Schieber and Williams indicates that the magnitude of age-related reductions in contrast sensitivity assessed under nighttime glare conditions may be greater for the low spatial frequency components of a visual stimulus than for their high frequency counterparts. ${ }^{(115)}$

\section{Spatial Resolution}

Acuity. With increasing age, there are significant declines in acuity among healthy older adults that are particularly prominent in low or poor lighting conditions. When Feree, Rand, and Lewis compared acuity as a function of illumination (5.4 to 108 lux) in a young ( 25 to 27 years) and an old group (42 to 62 years), the older observers required higher levels of illumination to reach any given acuity level. ${ }^{(116)}$ Their acuity also improved more as illumination was increased. Weston 
presented 12 subjects of different ages with a timed Landolt-C task that required participants to cancel targets with a particular orientation where target size and illumination were both varied. ${ }^{(117)}$ Although the oldest participant was only 48 years of age, at no point did the performance of the older group equal that of the young. As illuminance was increased, however, the performance of the older group increased more than that of the young, reducing the difference between the two groups. Rice and Jones examined acuity for over 4,000 drivers under both day and nighttime conditions. ${ }^{(18)}$ Failure to meet a 20/40 cutoff occurred with disproportionate frequency among older drivers on the nighttime test. Large age-related losses in acuity as a function of decreased luminance have also been reported by Richards; Sturgis, and Osgood; and Vola, Cornu, Carrvel, Gastaud, and Leid. ${ }^{(99,119,120)}$ For example, Vola et al. examined photopic $(\mathrm{I} 100 \mathrm{~cd} / \mathrm{m} 2)$ and mesopic $(0.8 \mathrm{~cd} / \mathrm{m} 2)$ acuity in 221 persons aged 20 through $50{ }^{(120)}$ No age differences in photopic acuity were observed, but mesopic acuity was found to decline significantly across middle age.

Most of the studies of age differences in the relationship between acuity and light level have used a relatively modest range of illumination. Consequently, there have not been sufficient data to allow an effective assessment of the ability of traditional laboratory/clinical measures of photopic acuity to predict the problems of older persons in low light, nor have the potential benefits of increased light to the older eye been explored fully. Sturr and Taub addressed this issue by comparing the proportion of young (18 to 25 years) and old (60 to 87 years) drivers who could meet a 20/40 acuity standard across six different luminance levels from 0.2 to 245.5 $\mathrm{cd} / \mathrm{m}^{2}{ }^{2}{ }^{121)}$ As luminance decreased so too did acuity, particularly among the older drivers. There was no difference between the young subjects and those aged 60 to 64 in the proportion able to pass a 20/40 acuity cutoff, but the proportion of those 65 and over who failed to meet the $20 / 40$ level increased directly with age. For example, at $2.4 \mathrm{~cd} / \mathrm{m}^{2}$, almost 77 percent of those under 65 possessed an acuity of 20/40 or better; the corresponding proportions were 28 percent for 65 to 75 -year-olds and 4 percent among those over 75 . At $0.78 \mathrm{~cd} / \mathrm{m}^{2}$, no observer over 65 was able to reach the $20 / 40$ criterion, even though over 36 percent of those aged 18 to 25 and more than 15 percent of those aged 60 to 64 were able to do so. Only about 30 percent of the older subjects were aware of their difficulties in seeing after sunset. Also, daytime acuity was found to be a poor predictor of low-illumination acuity.

Age and Contrast Sensitivity. By determining the contrast required to detect bar gratings varying in size (i.e., in spatial frequency), the contrast sensitivity function (CSF) provides a more comprehensive summary of an observer's spatial abilities than does acuity. Complex changes in the contrast sensitivity function are observed as luminance decreases from high to low photopic levels. Sensitivity for high spatial frequencies (small detail) is lost initially; peak sensitivity is shifted to lower spatial frequencies as luminance approaches the mesopic level; and sensitivity for low spatial frequency targets is preserved until very low levels of light are reached. ${ }^{(122)}$ With increasing age, there is little difference in the CSF at low spatial frequencies; a significant deficit, however, does appear at intermediate and high spatial frequencies. (See references 114, 123, 124, 125) When Owsley, Sekuler, and Siemsen measured contrast sensitivity among healthy adult subjects refracted to the test distance, they found that sensitivity to high spatial frequency gratings decreased beginning in the fifth decade. ${ }^{(125)}$ When the contrast sensitivity functions were established for young subjects who viewed the display through a 0.5 neutral density filter to approximate the reduction in retinal illuminance at age 60 , the difference between them and 
60-year-old observers was greatly reduced, leading the authors to conclude that a significant part of the sensitivity loss at intermediate and high spatial frequencies was due to reduced retinal illuminance.

Intra-ocular scatter and neural factors have also been implicated in the age-related loss of contrast sensitivity. ${ }^{(114,126)}$ Regardless of its cause, it is clear that older persons need higher levels of contrast when carrying out real-world visual tasks such as identifying common objects or highway signs. ${ }^{(127,128)}$

Visual Fields. The visual field, or area over which effective sight is maintained relative to a straight-ahead fixation point, declines with age and appears to have a deleterious effect on driving performance. Johnson and Keltner found that the frequency of significant field loss began to accelerate past age $60 .^{(129)}$ Drivers with a significant binocular visual field loss exhibited traffic accident and conviction rates that were twice as high as that of age and sex matched observers with normal fields.

\section{Spatiotemporal Resolution}

Dynamic Visual Acuity (DVA) Although not extensive, the research on DVA indicates that age differences in the resolution of spatial detail are more pronounced when the target is in motion relative to the observer than when it is stationary. (See references 130,131, 132, 133.) Although static visual acuity (SVA) sets the upper bound for spatial resolution on DVA tasks, the relationship between the two measures decreases as target motion increases, particularly among older observers. ${ }^{(110,132,134)}$

Despite its apparent predictive power for real-world tasks such as driving, little research has been carried out to identify the mechanisms that underlie the age-associated decline in DVA. $^{(110)}$ Although the inability of the eyes to track fast-moving targets offers the best explanation as to why DVA is worse than its static counterpart, age differences in DVA do not seem to be due to the age-related decline that occurs in the smooth pursuit system. ${ }^{(95,135)}$ Reading has suggested that dioptric factors are of greater importance than oculomotor ones in the DVA deficit, a conclusion supported in a recent study. ${ }^{(133,136)}$ Long and Crambert found large age differences in DVA even when stimulus durations were so brief that the possibility of pursuit eye movements was precluded. ${ }^{(136)}$ It is known that DVA improves with increasing luminance well above levels that would be asymptotic for SVA. ${ }^{(137)}$ The possibility that dynamic acuity of older observers might, like their static acuity, benefit significantly from elevations in luminance and contrast has not been examined.

Temporal Contrast Sensitivity. Studies of age differences in the rate of temporal contrast modulation that can be resolved have been somewhat inconsistent in their findings. Some studies have not found age differences in temporal contrast sensitivity; other investigations have indicated that the temporal contrast sensitivity of older observers is impaired at higher temporal frequencies. ${ }^{(138,139,140)}$ Mayer et al. compared the photopic temporal contrast sensitivity functions of observers aged 65 years and older with those of a young group matched for pupil size by means of artificial pupils. ${ }^{(139)}$ The contrast sensitivities of the older observers were lower than those of the younger observers, especially between 10 and $45 \mathrm{~Hz}$. The authors suggested that the difference reflected diminished sensitivity, not an age-related loss of temporal resolution. 
Others, however, have argued that such temporal losses reflect a slowing or change in the time constant of the neural response. ${ }^{(141,142)}$

Self-reported Visual Problems. The problems that older drivers experience in using visual displays are highly consistent with age-related changes in the visual system. Yee surveyed 446 drivers over 55 and found, that among their other problems, they reported particular difficulty in conditions of low illumination and with highway design, signs, and markers. ${ }^{(11)}$ In an extensive study of the relationships between driving performance and a variety of sensory, cognitive, psychomotor measures, Laux and Brelsford asked 104 drivers aged 40 to 92 about their visual ability and driving experiences. ${ }^{(9)}$ Although not significant, due perhaps to the relatively low number of drivers in each age group, older drivers were more likely to report difficulty seeing at night, to be bothered by oncoming headlight glare, and to have difficulty reading both their instrument panel and traffic signs. Older drivers were also more likely than their younger counterparts to report that they avoided night driving. When Kline et al. surveyed 400 drivers regarding their visual problems, they found 8 that were strongly age-related: seeing through glare on the windshield; seeing past windshield haze; seeing the instrument panel because it was too dim; judging the speed of their own vehicle; judging the speed of other vehicles as being too great; being surprised by another vehicle when merging; being surprised by unexpected vehicles in their peripheral vision; and reading signs in time to react to them appropriately.(10) These authors noted that the visual problems reported by older drivers were consistent with their reduced nighttime driving, as well as with the type of accidents that they were more likely to experience, such as failure to yield right-of-way, and the failure to heed signs. There is also some evidence that the visual problems of older drivers is related to their decision to stop driving. ${ }^{(143)}$

\section{Attention, Cognition, And Psychomotor Change}

Attention And Visual Search. Attention is frequently categorized into four main types: divided attention involving attending to two or more tasks simultaneously; attention switching where the person must alternate between two or more inputs; sustained attention involving the maintenance of attention over an extended duration; and selective attention in which a person must filter out irrelevant stimuli in favor of goal-relevant information. ${ }^{(69)}$ The available evidence indicates that an age deficit in divided attention occurs in all but the most simple of tasks. ${ }^{(144)}$ Most studies of attention switching, however, have not found an age difference. ${ }^{(69)}$ Yet, recent studies suggest that limitations in attention switching ability are closely related to impairment of driving ability in persons in the earliest stages of dementia. ${ }^{(145)}$ With respect to age changes in sustained attention, the data are mixed; although older drivers show lower levels of detection accuracy, there is little evidence that the age difference is greater with increased time on task. ${ }^{(69)}$ Lastly, there does appear to be a significant decline with age in selective attention. On average, older observers appear to be less able to attend to a single input in the presence of competing stimuli, perhaps because the irrelevant information has to be processed as part of the task, or because they have greater difficulty in discriminating between relevant and irrelevant stimuli. ${ }^{(146,147)}$ The spatial localization hypothesis of Plude and Hoyer holds that the age-related decline in selective attention is due to a decline in the ability to locate task-relevant information in the visual field, an argument that is consistent with recent research on age differences in the "useful field of view." 
The useful field of view, an attention measure involving the extent over which peripherally-presented complex visual stimuli can be identified and/or localized, appears to decline markedly with age. ${ }^{(148,149)}$ There is also evidence that it provides a better estimate of the difficulties that older persons have with peripherally-located real-world targets than do traditional field size measurements which assess only the presence of very simple stimuli. ${ }^{(150)}$ In fact, a recent study assessing useful field of view and mental status has reported a much stronger relationship between those measures and the automobile accidents of older drivers than those reported in prior studies of vision and driving. ${ }^{(151)}$

Psychomotor Performance. One of the most fundamental behavioral deficits of old age is a slowing in behavior. ${ }^{(152)}$ This can be seen in both the latency with which responses are initiated and in the speed with which they are carried out. Age-related slowing becomes more evident as task difficulty or complexity is increased, where non-verbal information processing is involved or if stimulus-response compatibility is lowered. ${ }^{(74)}$ It is also the case, however, that age-related declines in speed are minimized on continuously practiced skills. ${ }^{(153)}$

Intellectual Functioning. Although normal aging appears to spare such "crystallized" forms of intelligence as general information and vocabulary, laboratory measures of fluid or performance intelligence typically decline. Relatedly, there are marked reductions in the rate of information processing in old age. ${ }^{(65)}$ Research also shows that some of this decline can be reduced by increased levels of experience and a supportive environment. ${ }^{(154)}$ Salthouse has also pointed out that conventional tests of intellectual and cognitive functioning are often poor predictors of an older person's "cognitive competence" for real-world tasks. ${ }^{(155)}$

Memory. With regard to memory function, age differences appear to be greater for explicit memory, which requires an intention to remember, than for implicit memory, which occurs without such an intention. Although there is not much age decline in short-term memory generally, when the task depends on "working memory," which involves an active manipulation of information, or the dividing of attention, age deficits are more prominent. ${ }^{(67)}$

\section{AGING AND HIGHWAY SIGN EFFECTIVENESS}

In addition to the various sign characteristics, environmental factors, and driver variables already discussed, there are a number of human conditions that can influence the older driver's ability to acquire, understand, and react to traffic sign information. It is known that older persons are frequent users of licit drugs, many of which can reduce functioning of sensory systems, attention, or memory. The extent to which the use of such drugs is a problem in older drivers' acquiring traffic sign information is unknown. Although the use of alcohol among older drivers is much less of a problem than among the young and middle aged, it could still play a role by impeding the processing of information from traffic signs. Alcohol has been shown to impair perceptual and information processing abilities. ${ }^{(156)}$ One study showed that drivers at a 0.13 blood alcohol level had to be 13 percent closer to traffic signs in order to read them at night. ${ }^{(157)}$

The effects of fatigue on driving performance have been the subject of a number of studies. However, these have not specifically addressed the topic of traffic sign perception. Nevertheless, it seems likely that tired drivers would be less likely to detect signs and to process and act quickly upon relevant information than would alert drivers. 
Another set of factors particularly relevant to the older driver is the various physical health problems that they experience. To the extent that these detract from the driving task or reduce the physical or perceptual/cognitive skills required for information processing, they make it difficult for drivers to acquire and use traffic sign information.

In spite of the fact that there has been a great deal of research done on traffic signs, little is known about their effectiveness for older drivers. Age has been a variable in a few studies of sign comprehension, legibility distance and sign luminance requirements, but a thorough examination of the issue with a large sample of subjects and a wide variety of symbols has not been done to date. The following part of this review will summarize the main work on traffic signs where age has been one of the variables studied.

Olson, Sivak, and Egan noted the following potential factors that might reduce the legibility of traffic signs by older drivers: ${ }^{(158)}$

- They require more contrast between a sign message and its background.

- Legibility losses with age are greater under conditions of low background luminance.

- Legibility loss is greater for older subjects when luminance is increased beyond the optimum level on a partially reflectorized sign. If sign brightness at night is very high, the problem of irradiation (considered to be greater for the older driver) makes a sign message more difficult to read. In other words, brighter is better only up to a point.

In a major research effort aimed at relating older driver capacity to traffic control device design, Staplin, Lococo, and Sim examined information processing capabilities of young, middle-aged, and older drivers. ${ }^{(58)}$ Information gathered from a review of the literature and from focus groups led to the identification of specific difficulties older drivers have with traffic control devices. A battery of tests was then used to measure the most important sensory/perceptual, cognitive, and psychomotor abilities. Measurements were made of photopic and mesopic contrast sensitivity, digit span (an index of ability to store and manipulate items in short-term memory), a Stroop test (a measure of ability to ignore irrelevant visual stimuli), trail making ability (a measure of accuracy and flexibility of directed visual search), and compensatory tracking (a simulation of the cognitive, perceptual-motor integration demands involved in steering) Ophthalmological examination and clinical vision assessments were also performed. The results of these tests conformed with previous research that showed poorer performance by older drivers on such abilities.

The second phase of this project involved having groups of young/middle-aged (mean age 36.1 years) and older subjects (mean age 70.4) participate in a series of laboratory simulation experiments aimed at gauging performance in the use of traffic control devices, particularly signs. In the first sign study, the legibility of text signs was examined when legend size, setback distance from the roadway, and glare (present/absent) were manipulated. Larger letter sizes were required for older drivers to read the signs, but the glare condition produced only a slight decrement in legibility for the older drivers, and none for the young/middle-aged group (possibly because the veiling luminance experienced by the drivers was not very intense) Setback distance of the signs from the roadway made little difference in performance and did not interact with age. 
The correlations between contrast sensitivity and character size required for reading the signs accounted for about 24 percent of the variance in this measure under the least favorable viewing condition-the lowest background and lowest stimulus luminance levels. Under better viewing conditions, the correlations were lower. This finding underlines the importance of contrast sensitivity in identifying targets in low illumination. One general finding, consistent with other research, was the greater variability in performance among the older subjects. In an analytic study using a computer model it was found that legibility of signs was reduced much more by glare for 75-year-old drivers than for those aged 25 .

Additional experiments in this series found that older drivers are slower at making correct navigational decisions at freeway exits when given different amounts of information, in short term memory, required for the decision. In addition, they were slower at making decisions concerning whether they could turn left at intersections when provided specific traffic control device information, and less likely to notice (under conditions of brief exposure) certain types of signs-white/rectangular, yellow/diamond and yellow/pentagon.

A final set of studies by Staplin, et al., applied findings of their previous research to the design of traffic control device alternatives to accommodate the diminishing capabilities of older drivers. ${ }^{(58)}$ Performance of older drivers in simulated driving situations was improved by more appropriate sign size and placement, by repeating information on changeable message signs, and by providing additional sign information upstream to indicate "decision rules" for protected versus unprotected turns at intersections.

\section{Aging And Sign Luminance}

Although current standards for minimum display size and lighting seem to be appropriate for most young drivers, there is considerable evidence that displays used by older persons need to be larger, brighter and/or higher in contrast. Mourant and Langolf determined the ability of drivers aged 45 to 67 years to identify and respond to vehicle control legends ( $81 \mathrm{~cm} \mathrm{(31.89} \mathrm{in)}$ viewing distance) as a function of luminance, letter size and contrast under nighttime viewing conditions. ${ }^{(160)}$ They concluded that older drivers need 10 times as much light as younger ones to compensate for age-related losses in light and contrast sensitivity. Many of the older drivers were unable to identify legends composed of small $(0.23$ and $0.43 \mathrm{~cm}$ letter height $)$ low-contrast ratio $(1.25: 1,2: 1)$ letters at even the highest luminance tested $\left(68.2 \mathrm{~cd} / \mathrm{m}^{2}\right)$. Response times declined as contrast, letter size, and luminance were increased. Given that the high luminance levels needed by older drivers to assure the legibility of smaller legends at night would be uncomfortable or disabling under the mesopic luminance conditions that characterize night driving, the authors recommended the use of large (at least $0.64 \mathrm{~cm}(1.62 \mathrm{in})$ or 27 minarc) highcontrast letters on instrument panels.

Sivak, Olson, and Pastalan examined the effects of age on legibility of traffic signs at night by having subjects drive toward signs containing an $\mathrm{E}$ or a left/right reversal of this letter and identify the orientation of the 1etter. ${ }^{(161)}$ White letters appeared on green, red, blue, and black backgrounds, while the letter was black on white, yellow, and orange signs, thus providing a wide variation in contrast between target and background. Legibility distances for older drivers (over 61) were 65 percent to 77 percent of those of younger (under 25) drivers with comparable high luminance level visual acuity. The contrast ratios between letter and background varied 
from 1.5:1 (red on white with reflective sign panel) to 10000:1 (white on black with highly retroreflective buttons for the letters) Legibility distance was greatest for both groups at a contrast ratio of about 10: 1. A very high contrast is not optimal, especially for older drivers.

Rather similar conclusions to those of Mourant and Langoff were reached by Staplin, Lococo, Sim, and Drapcho, who determined the relationships between Landolt $\mathrm{C}$ contrast sensitivity and performance on two tasks (roadway heading discrimination and minimum letter size to read novel words and messages on traffic signs). ${ }^{(14,160)}$ They tested two age groups-a young group aged 18 to 49, and an old group aged 65 to 80 (half of them regular volunteers, half a "cross-validation sample" solicited at photo license centers). They found that age differences in contrast sensitivity increased as luminance decreased, especially among the cross-validation sample. Glare increased the contrast needed for the heading discrimination task, particularly among the old drivers in the greatest viewing distance condition. Correlations between contrast sensitivity and contrast needed for headway delineation were generally low, suggesting to the authors the importance of non-sensory factors in a driver's discrimination of roadway heading. Older drivers also required larger letter heights for word and message legibility. Older subjects had slightly more difficulty reading sign messages under the lowest luminance level, but the age differences were not statistically significant. Correlations between contrast sensitivity and word/message legibility were considerably higher than for heading discrimination, indicative of the greater relative importance of sensory function in this task. The authors noted that the tremendous variability among the older participants, as well as the larger differences between their volunteer and cross-validation older participants, posed a significant challenge for the design of traffic control devices. For example, although the older driver at the 50th percentile may need 2 to 2.5 times more contrast than the young driver, one in the lowest quartile may require 10 to 20 times as much.

\section{Aging And Symbol Signs}

Cooper used home interviews to examine comprehension of 46 traffic sign symbols in Britain. $^{(159)}$ The sample was composed of 1181 drivers, 323 non-drivers, and 122 children aged 10 to 15 . The level of correct comprehension ranged from 18 to 100 percent, with an overall average of 75 percent correct or partially correct. There was a significant effect of age, with those subjects 65 and older doing worst. The oldest non-drivers did worse than the children. The level of performance for the oldest group of drivers was about 15 percent worse than that for the best group (those 25 to 34). As expected, comprehension by drivers was well above that of non-drivers at all ages.

One of the Staplin, et al., experiments compared the same six traffic sign messages in both text and symbol formats. ${ }^{(58)}$ Drivers were shown a set of three signs one at a time and asked to indicate as quickly as possible whether one of these had the same message as a probe stimulus-horizontally written traffic sign message-shown after the third sign in the set. During this task, subjects were also performing a subsidiary compensatory tracking task of either low or high difficulty level. The older subject group was consistently poorer in terms of percent correct responses and mean reaction time. Surprisingly, performance was better on the text than on the symbol signs. The authors point out that this could be attributable to the specific matching task used-a printed match to a printed stimulus would be faster than such a match to a symbol stimulus. 
One of the few traffic sign studies that analyzed the data by age was done for the American Automobile Association (AAA) by Hulbert, Beers, and Fowler. ${ }^{(56)}$ They examined comprehension of several traffic sign symbols, traffic signals, and pavement markings with a large sample (over 3100) of drivers from across the United States. The method used was presentation of the traffic control devices in a color film showing a dynamic roadway situation as the vehicle approached the device. A multiple choice answer format was used. Data were broken down by age category, under 24 years, 24 to 49, and 50 and over. The "older" driver was not examined. Although only eight symbols were studied, significant differences (with the older group doing worse) were found for all but one of the signs ("School Crossing"). The overall level of understanding for the older group was 72 percent, with a range of 16 percent to 93 percent, while the young and middle-age groups averaged 78 percent and 79 percent correct, respectively.

As part of this study the authors examined comprehension of five traffic signal symbols (a red $\mathrm{X}$ and colored arrows) The older subjects performed more poorly on four of the five symbols and better on one. These data suggest that older drivers do have more difficulty with traffic sign symbols and signal arrows.

In a followup study conducted for the AAA a year later, Hulbert and Fowler used the same procedure but tested a different set of traffic control devices, including five traffic sign symbols. ${ }^{(162)}$ Comprehension levels for the 19 devices (10 signs, 3 signals, and 6 pavement markings) was consistently lower for the older (over 50) subjects. Comprehension was generally poor (below 60 percent) for the symbols and the older subjects performed significantly worse than one or both of the other age groups on three of the five symbols.

In an experiment designed specifically to examine the effects of driver age on traffic sign symbol recognition, Allen, Parseghian, and Van Valkenburg used a driving simulator that provided steering and speed control over a dynamic highway scene containing 72 symbol traffic signs. ${ }^{(163)}$ Subjects "drove" the roadway before and immediately after training, and 1 week later. Each of the age groups ( 21 to 29,30 to 44,45 to 59 , and over 60 ) was divided into three subgroups which received different training regimes- a $n$ educational pamphlet explaining the meaning and nature of the signs, a review of each sign in the simulator with an educational plaque below it, and the combination of these. Initial knowledge of the symbol signs declined with age, however, after training there was a substantial increase in the level of the knowledge which was essentially constant across age groups. This suggests that older drivers can be effectively trained on the meanings of symbols. Recognition errors reduced from approximately 48 (out of 72) to 23 immediately following training for the oldest group. The corresponding figures for the youngest group were 30 and 4 . One week later, subjects had forgotten the meanings of 3 to 4 symbols on average, irrespective of their age. The authors explain that age differences in symbol recognition could be due in part to a "generational effect," with younger drivers having had more exposure to symbol signs through driver education and training.

Cairney, in an Australian study, measured comprehension of six symbol traffic signs and found that levels of understanding ranged from 40 percent ("Road Narrows") to 84 percent ("Pedestrian Crossing"). (164) Among the stimuli tested were five Australian "hybrid" signs-with both symbol and supplementary words--which had the words removed. Understanding of the "No U-Turn," "No Right Turn," and "Two-Way Traffic" messages was good (81 to 91 percent), 
but for "Do Not Enter" and "Keep Left," it was 31 percent and 41 percent, respectively. This demonstrated the importance, for some messages, of using a supplementary word message or "educational tab," especially when new symbols are introduced into a system. Age was examined as it related to overall performance on 19 traffic sign items (I I of which measured the comprehension of symbols) in the survey. Performance decreased systematically with age. The mean score (out of 19) was 13.16 for the 18- to 19 -year-olds and 9.39 for the over 65 age group.

The influence of age and sex on sign comprehension was examined by Laux and Mayer using two comprehension tests employed by the Texas Dept. of Public Safety. ${ }^{(165)}$ Subjects, aged 40 to 92, were shown black and white versions of 35 road signs and asked to indicate which of three statements correctly represented "what you should do at, or near each road sign." For example, the three choices to the "Yield" sign were: (a) yield to other traffic; (b) expect other traffic to yield to you; (c) always stop. If this was representative of the items in the tests, the answers would seem to be fairly obvious to an experienced driver. Visual acuity and cognitive functioning were also assessed, the latter with four subtests of the Wechsler Adult Intelligence Scale.

The average performance was 90 percent correct ( 84 percent on those that were strictly symbol signs), but this high level of performance is not surprising in view of the fact that 23 of the signs were word, or symbol plus word messages. Performance of females decreased with each decade, but that of males did not, and females' comprehension of the signs was worse overall. Age-related decrements were found on the cognitive and visual acuity measures, and all correlated significantly with performance on the sign comprehension test. An additional finding, not surprising, was that signs that were seen more frequently by the subjects were better understood. Although this study reported a reduction in sign comprehension with age for females, it tell us little about the comprehension level of symbols, since most of the signs had words on them.

The simulator study of symbols by Allen et al. allowed the determination of the "response distance" at which signs were recognized. ${ }^{(163)}$ This was a decreasing function of age, with the oldest group being able to recognize the symbols at about half the distance of the youngest group on the initial run. The significant interaction between age and training indicated that recognition distance increased by about 50 percent for the youngest group, but by only half that amount for the oldest group.

The authors calculated a regression equation to represent recognition distance in feet (262 $2.23 \mathrm{x}$ age $-1.9 \mathrm{x}$ errors). In this experiment, young subjects responded at approximately $80 \mathrm{~m}$ (262 ft) and required approximately 2 s processing time. Considering the response distance deterioration with age- $0.67 \mathrm{~m}(2.2 \mathrm{ft})$ per year-and the lower speed driven by the oldest subjects, "an additional sign recognition time penalty of $1.5 \mathrm{~s}$ can be computed for drivers in their seventies." The authors also report that recognition distance was not correlated with dynamic acuity.

Mergler and Zandi reported that the responses of both young and old subjects were faster if the task was to match a symbol to its corresponding text version than when the task order was reversed. ${ }^{(166)}$ Halpern found that the verbal responses of young subjects were equally fast to both symbol and text signs; older subjects responded more quickly to text signs. ${ }^{(167)}$ There are 
reasons, however, to question the appropriateness of verbal response-time measures when comparing symbol and text signs. As several investigators have noted, a verbal-labeling response is directly compatible with the format of text but not symbol signs. (See references 44, 166, 168, 169.) Ells and Dewar, and Halpern reduced, but did not eliminate the compatibility problem by having the subject respond "yes" or "no" as to whether a sign verbally identified by the researcher was matched by a subsequently presented sign. $(20,167)$ Another problem with an overt verbal response when evaluating signs is that such an act is not normally a part of the driver's response to a sign. In driving, the small indistinct image of an unfamiliar sign becomes larger and increasingly more visible until the driver can discern its message. Thus, a sign's legibility distance determines the time available to the driver to maneuver appropriately in response to a sign's message. By this metric, symbol signs appear to have a distinct advantage over their verbal "cousins."

The legibility distances of 22 symbol traffic warning signs used in the United States were evaluated in a laboratory study by Paniati with a sample of "young" (under 45; mean age of 33) and "old" (over 55; mean age of 61) drivers. ${ }^{(37)}$ Legibility distances (the distance at which the sign contents could be described) were about $2 / 3$ the distance for the older driver, as compared to the young, group. Younger drivers had longer legibility distances on all but one of the symbols ("Added Lane"). In general, distances were greater for those symbols that were visually simple in their design and less for those with fine details. Paniati also determined legibility distances for word and symbol versions of eight messages and found the distances to be on average 2.8 times as great for the latter, with a range from 4.4 times to no difference. There was also a significant age by sign type interaction, with the advantage of symbol signs being greater for the older drivers.

Kline, Ghali, Kline, and Brown also found that symbol signs have an advantage among middle-aged and older drivers. ${ }^{(19)}$ The legibility distances of text and symbol highway signs were compared for young, middle-aged, and older observers under day and dusk lighting conditions. No age differences were observed due perhaps to the visually selected samples studied. Symbol signs, however, were visible from approximately twice as far away as text signs for all three age groups, a difference that was more pronounced under dusk conditions. There were no age differences in the comprehension of symbol signs, but considerable variability from one symbol sign to another in the degree to which it was comprehended. Acuity was found to be a better predictor of the legibility distance of text than of symbol signs in both day and dusk conditions. A similar advantage of symbol signs among young, middle-aged, and older drivers has been observed in other studies. ${ }^{(57,60)}$

Kline and Fuchs examined the legibility distances of standard text traffic signs as well as standard and modified versions of symbol signs among young, middle-aged, and older drivers in a laboratory study using computer-generated stimuli. For all three age groups the standard symbol signs could be identified at about twice the distance as could the text signs. Legibility distances of the improved symbols, which were designed using an optical blur approach in order to avoid higher spatial frequency elements, exceed those of both the text and the standard symbol versions. Mean legibility distances were greater for both the young and the middle-aged drivers, as compared to the older drivers. 
Presumably, the legibility distance advantage of symbol signs is based on the large contours and contour separations used to compose them. Certainly, there is indirect evidence that it is their coarsely-structured (i.e., low-spatial frequency) format that gives them their advantage in legibility distance. A study by Evans and Ginsburg found that observer contrast sensitivity at two spatial frequencies $(1.5$ and $12 \mathrm{cpd})$ correlated more highly than did static visual acuity (SVA) with the minimum size at which two similar symbol signs could be discriminated by both young and old observers. $\left.{ }^{(127}\right)$ It should also be noted that there were several methodological and analytical shortcomings in the Evans and Ginsburg study: that there were only seven subjects in the older group; observers' acuity was not optically corrected to the test distance; and the minimum size of the symbol display was confounded with age differences in both response speed and response certainty. Also, subjects in the young and old groups were matched for acuity, which reduced the range of acuity present in the sample, in turn reducing the likelihood of observing a relationship between SVA and minimum display size. Lastly, the correlation with minimum display size was determined separately for each of the six spatial frequencies composing the CSF, providing it with a six-fold statistical advantage over the single acuity measure in the likelihood of observing a significant relationship. Nonetheless, acuity appears to be a better measure of legibility of word signs, and contrast sensitivity, a better predictor of the legibility of symbol signs. (See references $19,57,60,128$. )

\section{Aging And Sign Design}

The literature outlined above clearly indicates advantages of symbol traffic signs over those with word messages. In spite of the positive features of symbols, it is evident that many are poorly understood or difficult to identify under less than ideal visual conditions. In addition, there is substantial evidence that older drivers have difficulty with traffic signs of all types, including symbol ones. The design of traffic signs must take into account the decrements that older drivers experience in sensory, perceptual, cognitive, and psychomotor function. It is, therefore, essential to know more about how well older drivers detect, identify, and understand symbol traffic sign messages, including the effects of degraded visual input due to environmental factors such as low illumination and glare. 


\section{CHAPTER 3: DAYTIME LEGIBILITY DISTANCE OF SYMBOL HIGHWAY SIGNS}

The primary goals of this study were to: evaluate age differences in the daytime legibility distance of most standard United States symbol highway signs, and to determine the degree to which such differences are associated with age declines in static visual acuity and/or contrast sensitivity.

As discussed in the literature review in chapter 2, most studies have shown that the distance at which highway signs can be identified (i.e., their visibility distance or legibility distance) is significantly reduced among older drivers. Recent studies have affirmed the adverse effects of driver age on the visibility for both symbol and word-message signs. Some of this work has also suggested that age-related visual losses might have different effects on word versus symbol signs. For example, Kline and Fuchs found that, although the correlations between acuity and the daytime visibility of word signs were significant for young, middle-aged, and older drivers, acuity was not a significant predictor of the legibility of either standard or new customdesigned symbol signs within any of the three age groups they tested. ${ }^{(57)}$ The authors suggested that this was due spatial-domain differences between the acuity and symbol sign discrimination tasks. As they noted, acuity is a fine detail (i.e., high spatial frequency) task, while the legibility of symbol signs appears to be a function of their low spatial frequency characteristics. Schieber, Ghali, and Kline have provided evidence to support this contention. ${ }^{(60)}$ In a study of young, middle-aged, and older drivers, they found that symbol signs whose power spectrum favored low as opposed to high spatial frequencies were visible from greater distances, and also, that a composite measure from the contrast sensitivity function (CSF), the high spatial frequency cutoff at half peak amplitude, explained a much greater proportion of the age variance in legibility distance (41 percent) than did acuity (6 percent).

The present study determined the minimum angular size (i.e., distance) at which 85 standard United States symbol highway signs could be identified by young, middle-aged, and older male and female drivers. It was expected that legibility distances would be reduced among older drivers compared to their younger counterparts. No gender differences were anticipated. Given that participants were required to identify all the significant components of a sign at their smallest size, it was anticipated that both acuity and the high spatial frequency cutoff from the CSF would be related to legibility distance.

\section{METHOD}

\section{Subjects}

Three age groups of active, licensed, community-resident drivers participated in the study: a young group (6 men and 6 women), $\underline{M}$ age $=24.3$ years (range 20 to 31), a middle-aged group (6 men and 6 women), $\underline{\mathrm{M}}$ age $=49.3$ (range 42 to 59), and an older group ( 9 men and 9 women), $\underline{M}$ age $=66.8$ (range 60 to 76 ). More older drivers than young or middle-aged were included in the study due to the greater variability in performance typically observed both within and between older observers. The mean number of years of education was 15.2 years (range 12 to 18 ) for the young, 13.4 years (range 8 to 16) for the middle-aged, and 14.4 years (range 11 to $20)$ for the older. None of the possible age differences in education were significant $(p=.20)$. Overall, the participants were in good general health, although middle-aged (2 of 12) and older (8 of 18) participants were more likely than young ( 0 of 12$)$ ones to have a chronic illness or 
complaint. Middle-aged ( 1 of 12) and older (5 of 18) were also more likely than the young ( 0 of $12)$ to take one or more medications chronically. The visual health of the participants was also good; only one young, one middle-aged, and two older participants reported having any chronic visual problems. Mean binocular photopic $\left(110 \mathrm{~cd} / \mathrm{m}^{2}\right)$ Landolt $\mathrm{C}$ acuity levels, assessed at the test distance of $5.5 \mathrm{~m}(18 \mathrm{ft}$ ), were excellent: 0.74 minarc (range 0.5 to 1.56 ) for the young, 0.68 minarc (range 0.5 to 1.20 ) for the middle-aged, and 0.98 minarc (range 0.56 to 1.42 ) for the old participants, respectively $(1.0$ minarc $=6 / 6(20 / 20)$. The acuity levels of both the young $(\mathrm{p}<.05)$ and the middle-aged drivers $(\mathrm{p}<.01)$ exceeded those of the older, but were not significantly different from each other.

\section{Stimuli And Apparatus}

Contrast sensitivity was measured at spatial frequencies of 1.5, 3.0, 6.0, 12.0 and 18.0 c/deg using the Vistech VCTS 6500 far chart (Form A) at a viewing distance of $3.05 \mathrm{~m} \mathrm{(10} \mathrm{ft)}$ and a luminance of $150 \mathrm{~cd} / \mathrm{m}^{2}$. To enhance generalizability of the results to the natural environment, the acuity, contrast sensitivity, and sign visibility thresholds were determined using the participant's presenting distance correction.

The test stimuli consisted of digitized versions of 85 symbol signs (see appendix A) from the Manual of Uniform Traffic Control Devices. ${ }^{(3)}$ They were presented in their regulation colors on an Apple High-Resolution 330 mm (13 in) color monitor, driven by a Raster Ops 364 24-bit video board under control of a Macintosh II ci computer. White-area screen luminance was maintained at $77 \mathrm{~cd} / \mathrm{m}^{2}$. A light gray mask [700 mm $(27.5 \mathrm{in})$ wide by $43 \mathrm{~cm}$ (17 in) high],

maintained at $45 \mathrm{~cd} / \mathrm{m}^{2}$, was placed around the screen to minimize adaptation effects that otherwise might have occurred from shifts of gaze between the screen and the black background of the vision tunnel. Luminance levels of the acuity and contrast sensitivity charts were measured using a Minolta LS- 110 spot photometer; screen luminance of the monitor was established with a UDT model 61 -CRT photometer. Participants viewed the signs from behind a fixed chin rest to maintain proper head position at the $5.5 \mathrm{~m}(18 \mathrm{ft})$ viewing distance.

\section{Procedure}

Subjects initially completed an informed consent form (appendix B) and a driver experience questionnaire (appendix C). They were also asked to indicate their age, sex, type of visual correction used, visual problems, chronic illnesses, and medication used. After assessment of acuity and contrast sensitivity, subjects were adapted to the light level in the test setting for approximately $5 \mathrm{~min}$ as the sign visibility task was explained to them. They were then introduced to the task using a symbol practice sign that was not part of the test set (NO PEDESTRIANS: R9-3a). To simulate increases in a sign's visual angular size as it is approached by a driver, the size of a sign, initially too small to be described, was increased in 7 percent steps. After each step, observers were requested to identify all of the sign's features that they could; the smallest size at which the participant could describe the structure of all of the critical features of each sign, according to a pre-determined scoring criterion (see appendix D) was recorded as its threshold. The scoring key was based on an identification of the critical details in each sign that distinguished it from other signs, and which were necessary to convey its message completely. 
For example, the RAILROAD STATION sign was increased in size until observers were able to include the train, the station, and the figure of a person waiting, in their description.

Signs were presented in one of six predetermined random orders. In the young and middle-aged groups, one male and one female participant each received one of the orders. Among the older, where each age by sex group included three extra participants, three of the orders were repeated among the men and the other three orders were repeated among the women. All testing occurred in a single extended session with a 10-to-15 min rest period mid-way through the session. The presentation rate of the signs and the duration of the rest period was adjusted to the comfort level of each participant. Mean run times for the young, middle-aged, and older participants, including the rest period, were $2 \mathrm{~h}: 24 \mathrm{~min}, 2 \mathrm{~h}: 39 \mathrm{~min}$, and $2 \mathrm{~h}: 45 \mathrm{~min}$, respectively. Subjects received an honorarium of $\mathbf{\$ 1 0}$ an hour for their participation.

\section{RESULTS}

\section{Contrast Sensitivity}

An Age (3) x Gender (2) x Spatial Frequency ${ }^{(5)}$ mixed design Analysis of Variance (ANOVA) was carried out on log-transformed contrast sensitivity. No effects for gender were observed so the analysis was repeated collapsing across gender. The resulting Age $\mathrm{x}$ Spatial Frequency ANOVA revealed significant main effects for both age, $[\underline{F}(2,39)=8.46, \underline{p}<0.001]$ and spatial frequency $[\underline{F}(4,156)=102.51, \underline{p}<0.0011$. Their interaction approached, but did not reach, significance $(\mathrm{p}=0.08$ ). As can be seen in figure 1 , the mean log CSF's of all three age groups were within the bounds of VCTS 6500 population norms for observers aged 10 to 70 years. Pair-wise comparisons (Tukey HSD with Tukey-Kramer adjustment for unequal n's) indicated that the mean log CSF's of the young and middle-aged groups were not significantly different from each other, but the sensitivity of both groups exceeded that of the older $(\underline{p}<0.01)$. Pair-wise T-Test comparisons with Bonferroni correction on the adjacent spatial frequencies indicated significant differences between 1.5 and $3.0 \mathrm{c} / \mathrm{deg}(\mathrm{p}<0.01)$, between 6.0 and $12.0 \mathrm{c} / \mathrm{deg}$ $(\mathrm{p}<0.01)$, and between $12.0 \mathrm{c} / \mathrm{deg}$ and $18.0 \mathrm{c} / \mathrm{deg}(\mathrm{p}<0.01)$; the difference between 3.0 and 6.0 c/deg was not significant.

To evaluate the possibility that a single CSF measure could be used to predict the legibility distance of symbol signs, several composite measures were extracted from each individual's CSF: peak log contrast sensitivity of each observer ("peak sensitivity"), spatial frequency at peak of the $\log$ contrast sensitivity function ("peak spatial frequency," low spatial frequency at half of the peak amplitude of the log contrast sensitivity function ("low cutoff"), high spatial frequency at half of the peak amplitude of the log contrast sensitivity function ("high cutoff"), and the bandwidth of the log contrast sensitivity function at half peak amplitude ("bandwidth" = the difference between the high and low cutoff measures). For many observers, however, the VCTS contrast sensitivity function did not fall as low as half of its peak value on the low-spatial frequency end, making it impossible to derive the low cutoff or bandwidth measure from them (see table 1). No age differences were seen on the peak sensitivity measure, but the spatial frequency at which the peak occurred declined with age $[\underline{F}(2,39)=3.53, p<0.05]$. Tukey pairwise comparisons indicated that this was the result of a significant difference between the peak spatial frequency of young and older drivers; neither group was significantly different from the middle-aged. The high cutoff was also significantly lower among older drivers $[\underline{F}(2,39)=$ 
$7.154, \mathrm{p}<0.01$ ], an effect that pairwise comparisons indicated to be significant between both the young and older $(\mathrm{p}<0.01)$ and the middle-aged and older groups $(\mathrm{p}<0.02)$.

Table 1. Age differences in derived contrast sensitivity measures.

\begin{tabular}{lrrrr} 
& Young & $\underline{\text { Aged }}$ & $\underline{\text { Older }}$ & $\underline{\text { Age Diff }}$ \\
\cline { 2 - 5 } & 2.290 & 2.267 & 2.235 & none \\
Peak Sensitivity (Log CS) & 5.250 & 4.750 & 3.750 & a \\
Peak Spatial Frequency (c/deg) & 12.895 & 12.637 & 10.004 & ac \\
High Cutoff (c/deg) & & & \\
Note: & & \\
a = Significant difference $(\mathrm{p}<.05)$ between Young and Older Drivers \\
$\mathrm{c}=$ Significant difference $(\mathrm{p}<.05)$ between Middle-Aged and Older Drivers
\end{tabular}

\section{Sign Legibility Distance}

To estimate and compare the highway legibility distances of different signs, their threshold sizes were converted to legibility distances based on their regulation highway size using the formula: Legibility distance $(\mathrm{m})=($ Regulation Sign Size $(\mathrm{m}) /$ Threshold Size $(\mathrm{m}) \mathrm{x}$ Test Distance $(5.5 \mathrm{~m})$. An Age (3) x Gender (2) ANOVA on overall mean legibility distance indicated no significant gender effects, so a one-way ANOVA was carried out and that indicated a significant overall age effect $[\underline{F}(2,39)=3.53, \underline{p}<0.05]$. To identify the individual signs showing significant age differences, a series of one-way ANOVA's, followed by pair-wise comparisons was carried out on each sign. The results of these appear in table 2, along with the means and standard deviations for their legibility distances, ordered from highest to lowest on overall mean legibility distance.

The overall mean legibility distances (all age groups combined) in table 2, and in subsequent tables and figures with overall mean distances, are based on the mean of the means for the three age groups. It was considered more appropriate to report the overall means in this manner than as a weighted average based on the numbers of subjects in each age group. The latter approach would have biased the means towards the results of the oldest group, who represent the smallest proportion of drivers.

Age differences were also determined for the signs categorized into their different types: Warning, School, Guide, Regulatory, and Recreation/ Cultural (figure 2). One-way ANOVA's on each type showed a significant age difference for Warning $[\underline{F}(2,39)=5.31, \underline{p}<0.01]$; Guide $[\underline{F}(2,39)=6.89, \underline{p}<0.005]$; Regulatory $[\underline{F}(2,39)=4.67, \underline{p}<0.021$; and Recreational/Cultural $[\underline{F}(2,39)=10.42, \underline{p}<0.001]$ signs, but not for the School signs $(\underline{p}=0.39)$. Followup Tukey pair-wise corrections with Bonferroni correction indicated significant age differences between the older and the two younger groups for Warning, Guide, Regulatory and Recreation/ Cultural signs (table 3). Tables 4, 5, 6, and 7 show the legibility distances and the significance of age differences for the Warning, Regulatory, Guide, and Recreation/Cultural categories, respectively. Since the two School signs are also Warning signs, they are included in table 4. 


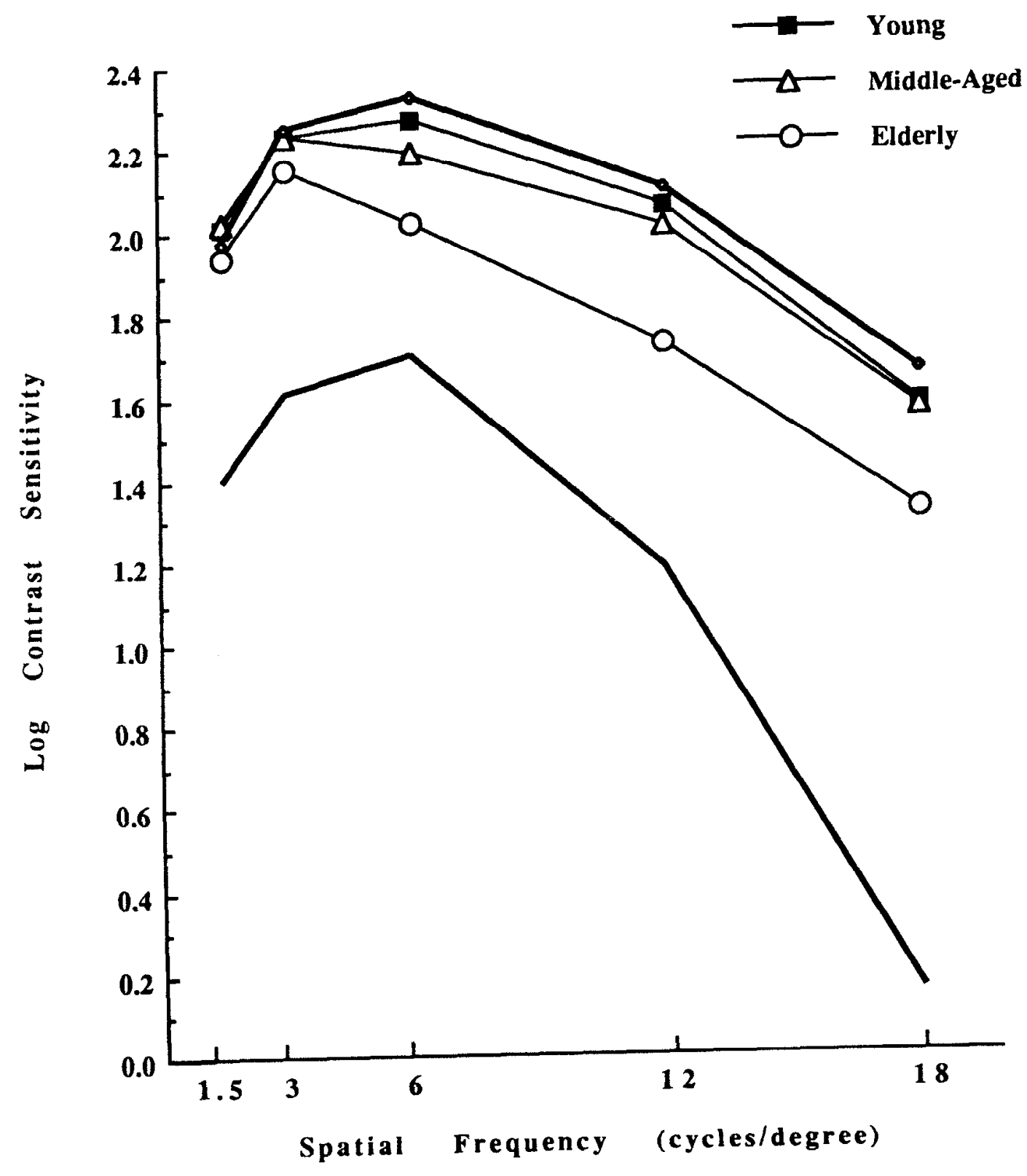

Figure 1. Log contrast sensitivity functions for young, middle-aged, and elderly drivers with VCTS norms (bold lines). 
Table 2. Mean legibility distance (m) and standard deviations (SD) of 85 symbol signs for young, middle-aged, and older drivers.

\begin{tabular}{|c|c|c|c|c|c|c|}
\hline $\begin{array}{l}\text { Rank } \\
\text { (Dist) }\end{array}$ & $\frac{\text { Sign Name }}{(\text { MUTCD No.) }}$ & $\begin{array}{l}\text { Age } \\
\text { Diff. }\end{array}$ & $\frac{\text { Young }}{(\mathrm{SD})}$ & $\begin{array}{l}\text { Middle- } \\
\text { Aged } \\
\underline{(\mathrm{SD})}\end{array}$ & $\frac{\text { Older }}{(\mathrm{SD})}$ & $\begin{array}{l}\text { Mean } \\
\text { Dist } \\
\underline{(\mathrm{SD})}\end{array}$ \\
\hline 1 & $\begin{array}{l}\text { Cross Road } \\
\text { (W2-1) }\end{array}$ & - & $\begin{array}{l}385.4 \\
(148.8)\end{array}$ & $\begin{array}{l}3 \overline{56.8} \\
(96.7)\end{array}$ & $\begin{array}{l}299.4 \\
(77.7)\end{array}$ & $\begin{array}{l}347.2 \\
(111.2)\end{array}$ \\
\hline 2. & $\begin{array}{l}\text { Added Lane } \\
\text { (W4-3) }\end{array}$ & $\mathrm{a}$ & $\begin{array}{l}363.9 \\
(\text { I } 16.4)\end{array}$ & $\begin{array}{l}343.0 \\
(96.7)\end{array}$ & $\begin{array}{l}267.7 \\
(63.9)\end{array}$ & $\begin{array}{l}324.9 \\
(98.7)\end{array}$ \\
\hline 3. & $\begin{array}{l}\text { Y Symbol } \\
\text { (W2-5) }\end{array}$ & - & $\begin{array}{c}321.0 \\
(111.0)\end{array}$ & $\begin{array}{l}311.5 \\
(87.8)\end{array}$ & $\begin{array}{l}269.8 \\
(67.7)\end{array}$ & $\begin{array}{l}300.8 \\
(88.5)\end{array}$ \\
\hline 4. & $\begin{array}{l}\text { Side Road (90 deg) } \\
\text { (W2-2) }\end{array}$ & - & $\begin{array}{l}328.7 \\
(109.4)\end{array}$ & $\begin{array}{l}305.6 \\
(74.7)\end{array}$ & $\begin{array}{l}263.6 \\
(61.5)\end{array}$ & $\begin{array}{l}299.3 \\
(84.1)\end{array}$ \\
\hline 5. & $\begin{array}{l}\text { Large Arrow } \\
\text { (W1-6) }\end{array}$ & $\mathrm{ac}$ & $\begin{array}{l}307.6 \\
(80.1)\end{array}$ & $\begin{array}{l}322.1 \\
(65.4)\end{array}$ & $\begin{array}{l}247.4 \\
(56.4)\end{array}$ & $\begin{array}{l}292.4 \\
(73.2)\end{array}$ \\
\hline 6. & $\begin{array}{l}\text { T Symbol } \\
\text { (W2-4) }\end{array}$ & - & $\begin{array}{l}310.2 \\
(98.9)\end{array}$ & $\begin{array}{l}300.3 \\
(89.1)\end{array}$ & $\begin{array}{l}258.3 \\
(60.7)\end{array}$ & $\begin{array}{l}289.6 \\
(82.8)\end{array}$ \\
\hline 7. & $\begin{array}{l}\text { Right Curve } \\
\text { (W1-2R) }\end{array}$ & - & $\begin{array}{l}270.5 \\
(87.5)\end{array}$ & $\begin{array}{l}310.1 \\
(68.7)\end{array}$ & $\begin{array}{l}269.1 \\
(49.4)\end{array}$ & $\begin{array}{l}283.2 \\
(68.4)\end{array}$ \\
\hline 8. & $\begin{array}{l}\text { Right Turn } \\
(\mathrm{W} 1-1 \mathrm{R})\end{array}$ & - & $\begin{array}{l}289.7 \\
(75.3)\end{array}$ & $\begin{array}{l}279.3 \\
(42.4)\end{array}$ & $\begin{array}{l}250.3 \\
(31.2)\end{array}$ & $\begin{array}{l}273.1 \\
(50.1)\end{array}$ \\
\hline 9. & $\begin{array}{l}\text { Right Reverse Curve } \\
\text { (W1-4R) }\end{array}$ & - & $\begin{array}{l}284.2 \\
(93.2)\end{array}$ & $\begin{array}{l}266.9 \\
(90.1)\end{array}$ & $\begin{array}{l}216.4 \\
(53.6)\end{array}$ & $\begin{array}{l}255.8 \\
(81.4)\end{array}$ \\
\hline 10 & $\begin{array}{l}\text { Double Head Arrow } \\
\text { (W1-7) }\end{array}$ & $\mathrm{ac}$ & $\begin{array}{l}268.7 \\
(88.2)\end{array}$ & $\begin{array}{l}290.1 \\
(55.9)\end{array}$ & $\begin{array}{l}205.4 \\
(53.5)\end{array}$ & $\begin{array}{l}254.7 \\
(74.5)\end{array}$ \\
\hline 11. & $\begin{array}{l}\text { Straight or Left } \\
\text { (R3-6) }\end{array}$ & - & $\begin{array}{l}254.0 \\
(67.3)\end{array}$ & $\begin{array}{l}247.1 \\
(56.7)\end{array}$ & $\begin{array}{l}248.5 \\
(63.0)\end{array}$ & $\begin{array}{l}249.9 \\
(61.1)\end{array}$ \\
\hline 12. & $\begin{array}{l}\text { Merge } \\
(\mathrm{W} 4-1)\end{array}$ & $\mathrm{ac}$ & $\begin{array}{l}276.2 \\
(69.4)\end{array}$ & $\begin{array}{l}263.8 \\
(60.6)\end{array}$ & $\begin{array}{l}196.6 \\
(54.1)\end{array}$ & $\begin{array}{l}245.5 \\
(69.7)\end{array}$ \\
\hline 13. & $\begin{array}{l}\text { Signal Ahead } \\
\text { (W3-3) }\end{array}$ & - & $\begin{array}{l}256.9 \\
(74.9)\end{array}$ & $\begin{array}{l}253.4 \\
(63.6)\end{array}$ & $\begin{array}{l}214.1 \\
(65.7)\end{array}$ & $\begin{array}{l}241.5 \\
(69.4)\end{array}$ \\
\hline 14. & $\begin{array}{l}\text { Side Road (45 deg) } \\
\text { (W2-3) }\end{array}$ & $c$ & $\begin{array}{l}259.3 \\
(93.9)\end{array}$ & $\begin{array}{l}266.9 \\
(71.6)\end{array}$ & $\begin{array}{l}196.4 \\
(63.2)\end{array}$ & $\begin{array}{l}240.9 \\
(80.8)\end{array}$ \\
\hline 15. & $\begin{array}{l}\text { Hospital } \\
\text { (D9-2) }\end{array}$ & $\mathrm{ac}$ & $\begin{array}{l}257.0 \\
(59.4)\end{array}$ & $\begin{array}{l}257.7 \\
(38.4)\end{array}$ & $\begin{array}{l}201.6 \\
(48.7)\end{array}$ & $\begin{array}{l}238.8 \\
(55.7)\end{array}$ \\
\hline 16. & $\begin{array}{l}\text { Tourist Information } \\
\text { (D9-10) }\end{array}$ & - & $\begin{array}{l}245.1 \\
(68.6)\end{array}$ & $\begin{array}{l}249.6 \\
(42.7)\end{array}$ & $\begin{array}{l}204.8 \\
(45.3)\end{array}$ & $\begin{array}{l}233.2 \\
(55.3)\end{array}$ \\
\hline
\end{tabular}

Notes:

$a=$ Significant difference $(p<.05)$ between Young and Older Drivers

$\mathrm{c}=$ Significant difference $(\mathrm{p}<.05)$ between Middle-Aged and Older Drivers 
Table 2. Mean legibility distance (m) and standard deviations (SD) of 85 symbol signs for young, middle-aged, and older drivers (continued).

\begin{tabular}{|c|c|c|c|c|c|c|}
\hline $\begin{array}{l}\text { Rank } \\
\text { (Dist) }\end{array}$ & $\begin{array}{l}\text { Sign Name } \\
\underline{\text { (MUTCD No.) }}\end{array}$ & $\begin{array}{l}\text { Age } \\
\text { Diff. }\end{array}$ & $\frac{\text { Young }}{\underline{(\mathrm{SD})}}$ & $\begin{array}{l}\text { Middle- } \\
\underline{\text { Aged }} \\
\underline{(\mathrm{SD})}\end{array}$ & $\begin{array}{l}\text { Older } \\
\underline{(\mathrm{SD})}\end{array}$ & $\begin{array}{c}\text { Mean } \\
\text { Dist } \\
(\mathrm{SD}) \\
\end{array}$ \\
\hline 17. & $\begin{array}{l}\text { Right Reverse Turn } \\
\text { (W1-3R) }\end{array}$ & - & $\begin{array}{l}273.5 \\
(148.8)\end{array}$ & $\begin{array}{l}225.0 \\
(47.7)\end{array}$ & $\begin{array}{l}197.7 \\
(51.3)\end{array}$ & $\begin{array}{l}232.1 \\
(93.0)\end{array}$ \\
\hline 18 & $\begin{array}{l}\text { Winding Road Right } \\
\text { (W1-5R) }\end{array}$ & - & $\begin{array}{l}252.0 \\
(91.0)\end{array}$ & $\begin{array}{l}242.9 \\
(72.0)\end{array}$ & $\begin{array}{l}200.3 \\
(65.0)\end{array}$ & $\begin{array}{l}231.7 \\
(77.0)\end{array}$ \\
\hline 19. & $\begin{array}{l}\text { Keep Right } \\
(\mathrm{R} 4-7)\end{array}$ & $\mathrm{a}$ & $\begin{array}{l}252.3 \\
(82.0)\end{array}$ & $\begin{array}{l}226.4 \\
(56.0)\end{array}$ & $\begin{array}{l}181.1 \\
(60.4)\end{array}$ & $\begin{array}{l}219.9 \\
(71.5)\end{array}$ \\
\hline 20. & $\begin{array}{l}\text { Airport } \\
(1-5)\end{array}$ & - & $\begin{array}{l}228.2 \\
(62.2)\end{array}$ & $\begin{array}{l}236.2 \\
(32.3)\end{array}$ & $\begin{array}{l}192.9 \\
(55.5)\end{array}$ & $\begin{array}{l}219.1 \\
(54.7)\end{array}$ \\
\hline 21. & $\begin{array}{l}\text { Lane Reduction } \\
\text { Transition } \\
\text { (W4-2) }\end{array}$ & - & $\begin{array}{l}246.4 \\
(108.4)\end{array}$ & $\begin{array}{l}214.8 \\
(75.9)\end{array}$ & $\begin{array}{l}195.4 \\
(53.9)\end{array}$ & $\begin{array}{l}218.9 \\
(79.7)\end{array}$ \\
\hline 22 & $\begin{array}{l}\text { Yield Ahead } \\
\text { (W3-2a) }\end{array}$ & $\mathrm{a}$ & $\begin{array}{l}264.1 \\
(107.8)\end{array}$ & $\begin{array}{l}224.6 \\
(66.9)\end{array}$ & $\begin{array}{l}165.0 \\
(48.3)\end{array}$ & $\begin{array}{l}217.9 \\
(84.2)\end{array}$ \\
\hline 23. & $\begin{array}{l}\text { Divided Highway } \\
\text { Begins (W6-1) }\end{array}$ & $\mathrm{a}$ & $\begin{array}{l}236.1 \\
(83.3)\end{array}$ & $\begin{array}{l}199.5 \\
(45.9)\end{array}$ & $\begin{array}{l}176.0 \\
(59.1)\end{array}$ & $\begin{array}{l}203.9 \\
(67.1)\end{array}$ \\
\hline 24. & $\begin{array}{l}\text { Two-Way Traffic } \\
\text { (W6-3) }\end{array}$ & - & $\begin{array}{l}208.0 \\
(71.8)\end{array}$ & $\begin{array}{l}217.9 \\
(42.8)\end{array}$ & $\begin{array}{l}180.0 \\
(50.6)\end{array}$ & $\begin{array}{l}202.0 \\
(56.8)\end{array}$ \\
\hline 25 & $\begin{array}{l}\text { Divided Highway } \\
\text { Ends } \\
\text { (W6-2) }\end{array}$ & - & $\begin{array}{l}228.4 \\
(91.2)\end{array}$ & $\begin{array}{l}196.5 \\
(40.2)\end{array}$ & $\begin{array}{l}177.9 \\
(65.2)\end{array}$ & $\begin{array}{l}200.9 \\
(69.9)\end{array}$ \\
\hline 26 & $\begin{array}{l}\text { School Advance } \\
(\mathrm{S} 1-1)\end{array}$ & - & $\begin{array}{l}202.8 \\
(61.9)\end{array}$ & $\begin{array}{l}215.3 \\
(58.6)\end{array}$ & $\begin{array}{l}184.4 \\
(39.0)\end{array}$ & $\begin{array}{l}200.8 \\
(52.5)\end{array}$ \\
\hline 27 & $\begin{array}{l}\text { Advance Flagger } \\
(\mathrm{W} 20-7 \mathrm{a})\end{array}$ & - & $\begin{array}{l}209.2 \\
(58.7)\end{array}$ & $\begin{array}{l}219.7 \\
(70.5)\end{array}$ & $\begin{array}{l}173.0 \\
(43.6)\end{array}$ & $\begin{array}{l}200.6 \\
(59.1)\end{array}$ \\
\hline 28 & $\begin{array}{l}\text { Phone } \\
\text { (D9-1) }\end{array}$ & - & $\begin{array}{l}212.4 \\
(59.5)\end{array}$ & $\begin{array}{l}203.2 \\
(48.0)\end{array}$ & $\begin{array}{l}176.9 \\
(45.8)\end{array}$ & $\begin{array}{l}197.5 \\
(51.9)\end{array}$ \\
\hline 29. & $\begin{array}{l}\text { Chevron Alignment } \\
\text { (W1-8) }\end{array}$ & $\mathrm{a}$ & $\begin{array}{l}223.8 \\
(67.1)\end{array}$ & $\begin{array}{l}202.9 \\
(52.3)\end{array}$ & $\begin{array}{l}165.8 \\
(38.7)\end{array}$ & $\begin{array}{l}197.5 \\
(56.5)\end{array}$ \\
\hline 30 . & $\begin{array}{l}\text { Camping } \\
\text { (D9-3) }\end{array}$ & - & $\begin{array}{l}215.2 \\
(73.8)\end{array}$ & $\begin{array}{l}206.1 \\
(91.6)\end{array}$ & $\begin{array}{l}167.8 \\
(50.1)\end{array}$ & $\begin{array}{l}196.4 \\
(72.3)\end{array}$ \\
\hline 31. & $\begin{array}{l}\text { Handicapped } \\
\text { (D9-6) }\end{array}$ & $\mathrm{ac}$ & $\begin{array}{l}213.6 \\
(62.7)\end{array}$ & $\begin{array}{l}211.3 \\
(57.9)\end{array}$ & $\begin{array}{l}161.2 \\
(49.5)\end{array}$ & $\begin{array}{l}195.4 \\
(60.2)\end{array}$ \\
\hline 32. & $\begin{array}{l}\text { Stop Ahead } \\
\text { (W3-1a) }\end{array}$ & $c$ & $\begin{array}{l}187.4 \\
(77.4)\end{array}$ & $\begin{array}{l}225.8 \\
(96.3)\end{array}$ & $\begin{array}{l}158.8 \\
(66.0)\end{array}$ & $\begin{array}{l}190.7 \\
(81.8)\end{array}$ \\
\hline
\end{tabular}


Table 2. Mean legibility distance (m) and standard deviations (SD) of 85 symbol signs for young, middle-aged, and older drivers (continued).

\begin{tabular}{|c|c|c|c|c|c|c|}
\hline $\begin{array}{l}\text { Rank } \\
\text { (Dist) }\end{array}$ & $\begin{array}{l}\text { Sign Name } \\
\text { (MUTCD No.) }\end{array}$ & $\begin{array}{l}\text { Age } \\
\text { Diff. }\end{array}$ & $\frac{\text { Young }}{\underline{(\mathrm{SD})}}$ & $\begin{array}{l}\text { Middle- } \\
\underline{\text { Aged }} \\
\underline{(\mathrm{SD})}\end{array}$ & $\frac{\text { Older }}{\underline{(\mathrm{SD})}}$ & $\begin{array}{c}\text { Mean } \\
\text { Dist } \\
\underline{(\mathrm{SD})}\end{array}$ \\
\hline 33 & $\begin{array}{l}\text { Slippery When Wet } \\
\text { (W8-5) }\end{array}$ & $\mathrm{c}$ & $\begin{array}{l}191.6 \\
(68.2)\end{array}$ & $\begin{array}{l}206.1 \\
(54.4)\end{array}$ & $\begin{array}{l}155.7 \\
(40.7)\end{array}$ & $\begin{array}{l}184.5 \\
(56.8)\end{array}$ \\
\hline 34. & $\begin{array}{l}\text { Deer Crossing } \\
\text { (W11-3) }\end{array}$ & - & $\begin{array}{l}190.3 \\
(76.3)\end{array}$ & 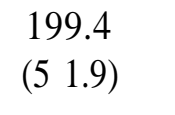 & $\begin{array}{l}162.9 \\
(46.9)\end{array}$ & $\begin{array}{l}184.2 \\
(58.9)\end{array}$ \\
\hline 35 & $\begin{array}{l}\text { Worker (Construction) } \\
\text { (W21-1a) }\end{array}$ & $\mathrm{a}$ & $\begin{array}{l}196.1 \\
(102.5)\end{array}$ & $\begin{array}{l}187.1 \\
(35.9)\end{array}$ & $\begin{array}{l}137.4 \\
(31.6)\end{array}$ & $\begin{array}{l}173.5 \\
(65.8)\end{array}$ \\
\hline 36 & $\begin{array}{l}\text { Dock } \\
(\mathrm{I}-9)\end{array}$ & $\mathrm{a}$ & $\begin{array}{l}186.7 \\
(54.0)\end{array}$ & $\begin{array}{l}167.4 \\
(28.8)\end{array}$ & $\begin{array}{l}150.0 \\
(30.2)\end{array}$ & $\begin{array}{l}168.0 \\
(40.2)\end{array}$ \\
\hline 37. & $\begin{array}{l}\text { Low Vertical } \\
\text { Clearance } \\
\text { (W12-2) }\end{array}$ & - & $\begin{array}{l}178.6 \\
(84.7)\end{array}$ & $\begin{array}{l}157.5 \\
(34.9)\end{array}$ & $\begin{array}{l}143.8 \\
(42.3)\end{array}$ & $\begin{array}{l}160.0 \\
(56.6)\end{array}$ \\
\hline 38 & $\begin{array}{l}\text { Pedestrian Crossing } \\
\text { (W11a-2) }\end{array}$ & $c$ & $\begin{array}{l}165.3 \\
(51.8)\end{array}$ & $\begin{array}{l}181.0 \\
(57.8)\end{array}$ & $\begin{array}{l}133.1 \\
(29.4)\end{array}$ & $\begin{array}{l}159.8 \\
(49.1)\end{array}$ \\
\hline 39. & $\begin{array}{l}\text { Cattle Crossing } \\
(\mathrm{W} 11-4)\end{array}$ & - & $\begin{array}{l}167.5 \\
(66.2)\end{array}$ & $\begin{array}{l}148.5 \\
(30.3)\end{array}$ & $\begin{array}{l}142.9 \\
(27.9)\end{array}$ & $\begin{array}{l}153.0 \\
(43.1)\end{array}$ \\
\hline 40 & $\begin{array}{l}\text { No Right Turn } \\
\text { (R3-1) }\end{array}$ & $\mathrm{a}$ & $\begin{array}{l}173.2 \\
(61.1)\end{array}$ & $\begin{array}{l}150.9 \\
(29.7)\end{array}$ & $\begin{array}{l}132.2 \\
(38.4)\end{array}$ & $\begin{array}{l}152.1 \\
(46.4)\end{array}$ \\
\hline 41. & $\begin{array}{l}\text { School Crossing } \\
(\mathrm{S} 2-1)\end{array}$ & $c$ & $\begin{array}{l}146.8 \\
(40.6)\end{array}$ & $\begin{array}{l}171.7 \\
(38.9)\end{array}$ & $\begin{array}{l}121.3 \\
(38.9)\end{array}$ & $\begin{array}{l}146.6 \\
(43.9)\end{array}$ \\
\hline 42. & $\begin{array}{l}\text { Bicycle Crossing } \\
\text { (W11-1) }\end{array}$ & $\mathrm{ac}$ & $\begin{array}{l}159.4 \\
(31.3)\end{array}$ & $\begin{array}{l}156.5 \\
(27.8)\end{array}$ & $\begin{array}{l}122.5 \\
(25.2)\end{array}$ & $\begin{array}{l}146.1 \\
(32.4)\end{array}$ \\
\hline 43. & $\begin{array}{l}\text { Railroad Advance } \\
\text { Warning } \\
\text { (W10-1) }\end{array}$ & - & $\begin{array}{l}140.6 \\
(49.0)\end{array}$ & $\begin{array}{l}157.9 \\
(57.2)\end{array}$ & $\begin{array}{l}123.4 \\
(34.4)\end{array}$ & $\begin{array}{l}140.6 \\
(47.2)\end{array}$ \\
\hline 44 & $\begin{array}{l}\text { Gas } \\
\text { (D9-7) }\end{array}$ & $\mathrm{a}$ & $\begin{array}{l}169.5 \\
(55.1)\end{array}$ & $\begin{array}{l}140.7 \\
(52.9)\end{array}$ & $\begin{array}{l}102.7 \\
(33.0)\end{array}$ & $\begin{array}{l}137.6 \\
(53.2)\end{array}$ \\
\hline 45 & $\begin{array}{l}\text { No U-Turn } \\
\text { (R3-4) }\end{array}$ & $\mathrm{a}$ & $\begin{array}{l}153.4 \\
(67.3)\end{array}$ & $\begin{array}{l}144.2 \\
(21.0)\end{array}$ & $\begin{array}{l}109.1 \\
(39.3)\end{array}$ & $\begin{array}{l}135.6 \\
(48.8)\end{array}$ \\
\hline 46. & $\begin{array}{l}\text { Swimming } \\
\text { (RW-130) }\end{array}$ & $\mathrm{ac}$ & $\begin{array}{l}153.6 \\
(40.9)\end{array}$ & $\begin{array}{l}144.3 \\
(19.9)\end{array}$ & $\begin{array}{l}104.1 \\
(25.6)\end{array}$ & $\begin{array}{l}134.0 \\
(36.7)\end{array}$ \\
\hline 47. & $\begin{array}{l}\text { No Parking } \\
\text { (R8-3a) }\end{array}$ & - & $\begin{array}{l}147.6 \\
(48.6)\end{array}$ & $\begin{array}{l}138.0 \\
(30.9)\end{array}$ & $\begin{array}{l}116.3 \\
(28.1)\end{array}$ & $\begin{array}{l}134.0 \\
(37.5)\end{array}$ \\
\hline 48 & $\begin{array}{l}\text { Narrow Bridge } \\
\text { (W5-2a) }\end{array}$ & $\mathrm{ac}$ & $\begin{array}{l}152.5 \\
(35.2)\end{array}$ & $\begin{array}{l}139.9 \\
(38.0)\end{array}$ & $\begin{array}{l}100.1 \\
(24.9)\end{array}$ & $\begin{array}{l}130.8 \\
(39.1)\end{array}$ \\
\hline
\end{tabular}


Table 2. Mean legibility distance (m) and standard deviations (SD) of 85 symbol signs for young, middle-aged, and older drivers (continued).

\begin{tabular}{|c|c|c|c|c|c|c|}
\hline $\begin{array}{l}\text { Rank } \\
\text { (Dist) }\end{array}$ & $\begin{array}{l}\frac{\text { Sign Name }}{(\text { MUTCD No.) }} \\
\end{array}$ & $\begin{array}{l}\text { Age } \\
\text { Diff. }\end{array}$ & $\begin{array}{c}\text { Young } \\
(\mathrm{SD})\end{array}$ & $\begin{array}{l}\text { Middle- } \\
\underline{\text { Aged }} \\
\underline{(\mathrm{SD})}\end{array}$ & $\begin{array}{l}\text { Older } \\
\underline{(\mathrm{SD})}\end{array}$ & $\begin{array}{l}\text { Mean } \\
\text { Dist } \\
\underline{(\mathrm{SD})}\end{array}$ \\
\hline 49. & $\begin{array}{l}\text { Tractor Crossing } \\
\text { (WI 1-5) }\end{array}$ & - & $\begin{array}{l}140.2 \\
(58.9)\end{array}$ & $\begin{array}{l}142.8 \\
(51.9)\end{array}$ & $\begin{array}{l}104.3 \\
(37.2)\end{array}$ & $\begin{array}{l}129.1 \\
(50.8)\end{array}$ \\
\hline 50 & $\begin{array}{l}\text { Hill } \\
\text { (W7-1) }\end{array}$ & $\mathrm{ac}$ & $\begin{array}{l}128.8 \\
(32.2)\end{array}$ & $\begin{array}{l}133.6 \\
(24.8)\end{array}$ & $\begin{array}{l}101.7 \\
(25.4)\end{array}$ & $\begin{array}{l}121.4 \\
(30.5)\end{array}$ \\
\hline 51. & $\begin{array}{l}\text { Bus Station } \\
\text { (I-6) }\end{array}$ & - & $\begin{array}{l}124.7 \\
(36.1)\end{array}$ & $\begin{array}{l}131.8 \\
(40.8)\end{array}$ & $\begin{array}{l}100.3 \\
(33.1)\end{array}$ & $\begin{array}{l}118.9 \\
(38.1)\end{array}$ \\
\hline 52 & $\begin{array}{l}\text { Recreational Vehicle } \\
\text { (D9-3a) }\end{array}$ & $\mathrm{ac}$ & $\begin{array}{l}131.1 \\
(49.1)\end{array}$ & $\begin{array}{l}127.5 \\
(35.2)\end{array}$ & $\begin{array}{l}95.2 \\
(23.5)\end{array}$ & $\begin{array}{l}117.9 \\
(38.8)\end{array}$ \\
\hline 53 & $\begin{array}{l}\text { Playground } \\
\text { (W15-1) }\end{array}$ & - & $\begin{array}{l}120.8 \\
(41.1)\end{array}$ & $\begin{array}{l}118.4 \\
(34.2)\end{array}$ & $\begin{array}{l}107.4 \\
(29.2)\end{array}$ & $\begin{array}{l}115.5 \\
(34.0)\end{array}$ \\
\hline 54 & $\begin{array}{l}\text { Double Arrow } \\
\text { (W12-1) }\end{array}$ & - & $\begin{array}{l}124.6 \\
(30.3)\end{array}$ & $\begin{array}{l}115.3 \\
(29.2)\end{array}$ & $\begin{array}{l}103.0 \\
(29.2)\end{array}$ & $\begin{array}{l}114.3 \\
(30.2)\end{array}$ \\
\hline 55 & $\begin{array}{l}\text { Propane } \\
\text { (D9-15) }\end{array}$ & $\mathrm{c}$ & $\begin{array}{l}114.7 \\
(35.9)\end{array}$ & $\begin{array}{l}121.7 \\
(30.1)\end{array}$ & $\begin{array}{r}95.9 \\
(15.9)\end{array}$ & $\begin{array}{l}110.8 \\
(28.7)\end{array}$ \\
\hline 56 & $\begin{array}{l}\text { No Trucks } \\
\text { (R5-2) }\end{array}$ & $\mathrm{c}$ & $\begin{array}{l}113.6 \\
(38.1)\end{array}$ & $\begin{array}{l}126.2 \\
(21.0)\end{array}$ & $\begin{array}{c}90.8 \\
(27.0)\end{array}$ & $\begin{array}{l}110.2 \\
(32.3)\end{array}$ \\
\hline 57 & $\begin{array}{l}\text { Restrooms } \\
\text { (RM-140) }\end{array}$ & $\mathrm{a}$ & $\begin{array}{l}123.2 \\
(42.8)\end{array}$ & $\begin{array}{l}106.7 \\
(32.0)\end{array}$ & $\begin{array}{c}90.8 \\
(28.8)\end{array}$ & $\begin{array}{l}106.9 \\
(36.0)\end{array}$ \\
\hline 58 & $\begin{array}{l}\text { National Network } \\
\text { Route } \\
\text { (R14-4) }\end{array}$ & $\mathrm{a}$ & $\begin{array}{l}114.2 \\
(35.1)\end{array}$ & $\begin{array}{l}110.2 \\
(17.8)\end{array}$ & $\begin{array}{r}91.4 \\
(16.2)\end{array}$ & $\begin{array}{l}105.3 \\
(25.2)\end{array}$ \\
\hline 59 & $\begin{array}{l}\text { Lodging } \\
\text { (D9-9) }\end{array}$ & $\mathrm{a}$ & $\begin{array}{l}118.4 \\
(17.1)\end{array}$ & $\begin{array}{l}106.7 \\
(22.2)\end{array}$ & $\begin{array}{c}90.5 \\
(18.4)\end{array}$ & $\begin{array}{l}105.2 \\
(22.2)\end{array}$ \\
\hline 60 & $\begin{array}{l}\text { Hiking Trail } \\
(\mathrm{I}-4)\end{array}$ & $\mathrm{a}$ & $\begin{array}{l}117.8 \\
(40.3)\end{array}$ & $\begin{array}{l}108.9 \\
(28.4)\end{array}$ & $\begin{array}{c}84.1 \\
(22.1)\end{array}$ & $\begin{array}{l}103.6 \\
(32.9)\end{array}$ \\
\hline 61. & $\begin{array}{l}\text { National Network } \\
\text { Route Prohibited } \\
\text { (R14-5) }\end{array}$ & - & $\begin{array}{l}107.3 \\
(40.0)\end{array}$ & $\begin{array}{l}102.2 \\
(30.8)\end{array}$ & $\begin{array}{l}92.8 \\
(33.5)\end{array}$ & $\begin{array}{l}100.8 \\
(34.5)\end{array}$ \\
\hline 62. & $\begin{array}{l}\text { Railroad Parallel to } \\
\text { Roadway } \\
\text { (W10-3) }\end{array}$ & $\mathrm{ac}$ & $\begin{array}{l}114.0 \\
(16.2)\end{array}$ & $\begin{array}{l}101.8 \\
(23.1)\end{array}$ & $\begin{array}{c}80.9 \\
(17.1)\end{array}$ & $\begin{array}{l}98.9 \\
(23.2)\end{array}$ \\
\hline 63. & $\begin{array}{l}\text { Canoeing } \\
\text { (RW-020) }\end{array}$ & $\mathrm{a}$ & $\begin{array}{l}113.6 \\
(38.1)\end{array}$ & $\begin{array}{c}93.8 \\
(24.8)\end{array}$ & $\begin{array}{c}75.5 \\
(23.1)\end{array}$ & $\begin{array}{c}94.3 \\
(32.1)\end{array}$ \\
\hline
\end{tabular}


Table 2. Mean legibility distance (m) and standard deviations (SD) of 85 symbol signs for young, middle-aged, and older drivers (continued).

\begin{tabular}{|c|c|c|c|c|c|c|}
\hline $\begin{array}{l}\text { Rank } \\
\text { (Dist) }\end{array}$ & $\begin{array}{r}\begin{array}{r}\text { Sign Name } \\
(\text { MUTCD No.) }\end{array} \\
\end{array}$ & $\begin{array}{l}\text { Age } \\
\text { Diff. }\end{array}$ & $\frac{\text { Young }}{(\mathrm{SD})}$ & $\begin{array}{l}\text { Middle- } \\
\underline{\text { Aged }} \\
\underline{(\mathrm{SD})}\end{array}$ & $\begin{array}{l}\text { Older } \\
(\mathrm{SD})\end{array}$ & $\begin{array}{c}\text { Mean } \\
\text { Dist } \\
\underline{(\mathrm{SD})}\end{array}$ \\
\hline 64 & $\begin{array}{l}\text { Snowmobiling } \\
\text { (RS-070) }\end{array}$ & $\mathrm{ac}$ & $\begin{array}{l}110.9 \\
(35.0)\end{array}$ & $\begin{array}{l}100.8 \\
(29.8)\end{array}$ & $\begin{array}{c}71.1 \\
(16.8)\end{array}$ & $\begin{array}{c}94.3 \\
(31.6)\end{array}$ \\
\hline 65 & $\begin{array}{l}\text { Pavement Ends } \\
(\mathrm{W} 8-3 \mathrm{a})\end{array}$ & $\mathrm{a}$ & $\begin{array}{l}109.9 \\
(47.1)\end{array}$ & $\begin{array}{l}96.5 \\
(27.6)\end{array}$ & $\begin{array}{c}75.0 \\
(13.2)\end{array}$ & $\begin{array}{l}93.8 \\
(33.2)\end{array}$ \\
\hline 66. & $\begin{array}{l}\text { Campfire } \\
\text { (RA-030) }\end{array}$ & $\mathrm{ac}$ & $\begin{array}{c}98.3 \\
(23.2)\end{array}$ & $\begin{array}{l}101.0 \\
(21.7)\end{array}$ & $\begin{array}{c}79.5 \\
(12.1)\end{array}$ & $\begin{array}{c}92.9 \\
(20.8)\end{array}$ \\
\hline 67. & $\begin{array}{l}\text { No Bicycles } \\
\text { (R5-6) }\end{array}$ & $\mathrm{ac}$ & $\begin{array}{l}97.8 \\
(26.2)\end{array}$ & $\begin{array}{l}100.7 \\
(17.2)\end{array}$ & $\begin{array}{l}76.8 \\
(18.6)\end{array}$ & $\begin{array}{l}91.8 \\
(23.1)\end{array}$ \\
\hline 68. & $\begin{array}{l}\text { Recreational Vehicle } \\
\text { Sanitary Station } \\
\text { (D9-12) }\end{array}$ & $c$ & $\begin{array}{c}90.7 \\
(47.6)\end{array}$ & $\begin{array}{l}112.6 \\
(60.5)\end{array}$ & $\begin{array}{l}71.4 \\
(28.0)\end{array}$ & $\begin{array}{c}91.6 \\
(47.1)\end{array}$ \\
\hline 69. & $\begin{array}{l}\text { Winter Recreation } \\
\text { (I-1 0) }\end{array}$ & $\mathrm{a}$ & $\begin{array}{l}116.0 \\
(48.0)\end{array}$ & $\begin{array}{c}86.6 \\
(36.1)\end{array}$ & $\begin{array}{c}68.1 \\
(28.1)\end{array}$ & $\begin{array}{c}90.2 \\
(41.2)\end{array}$ \\
\hline 70 & $\begin{array}{l}\text { Library } \\
\text { (I-8) }\end{array}$ & - & $\begin{array}{l}97.4 \\
(47.9)\end{array}$ & $\begin{array}{c}93.7 \\
(19.3)\end{array}$ & $\begin{array}{l}71.9 \\
(30.9)\end{array}$ & $\begin{array}{l}87.7 \\
(35.4)\end{array}$ \\
\hline 71 & $\begin{array}{l}\text { Food } \\
\text { (D9-8) }\end{array}$ & - & $\begin{array}{l}89.9 \\
(33.6)\end{array}$ & $\begin{array}{l}89.9 \\
(28.9)\end{array}$ & $\begin{array}{l}79.5 \\
(26.6)\end{array}$ & $\begin{array}{l}86.4 \\
(29.1)\end{array}$ \\
\hline 72 & $\begin{array}{l}\text { Amphitheater } \\
\text { (RL-010) }\end{array}$ & $\mathrm{a}$ & $\begin{array}{l}100.0 \\
(32.7)\end{array}$ & $\begin{array}{c}82.4 \\
(16.3)\end{array}$ & $\begin{array}{c}71.7 \\
(17.8)\end{array}$ & $\begin{array}{l}84.7 \\
(25.1)\end{array}$ \\
\hline 73. & $\begin{array}{l}\text { Diesel c } \\
\text { (D9-11) }\end{array}$ & & $\begin{array}{l}86.4 \\
(34.4)\end{array}$ & $\begin{array}{l}91.5 \\
(27.7)\end{array}$ & $\begin{array}{c}65.8 \\
(16.7)\end{array}$ & $\begin{array}{l}81.2 \\
(27.9)\end{array}$ \\
\hline 74 & $\begin{array}{l}\text { Shelter (Sleeping) } \\
\text { (RA-110) }\end{array}$ & $\mathrm{a}$ & $\begin{array}{l}106.8 \\
(62.7)\end{array}$ & $\begin{array}{l}70.8 \\
(19.3)\end{array}$ & $\begin{array}{c}58.7 \\
(16.3)\end{array}$ & $\begin{array}{l}78.8 \\
(41.0)\end{array}$ \\
\hline 75 & $\begin{array}{l}\text { Rest Area } \\
\text { (D5-5a) }\end{array}$ & $\mathrm{ac}$ & $\begin{array}{c}83.6 \\
(19.9)\end{array}$ & $\begin{array}{l}82.4 \\
(10.1)\end{array}$ & $\begin{array}{c}65.8 \\
(12.4)\end{array}$ & $\begin{array}{l}77.3 \\
(16.5)\end{array}$ \\
\hline 76 & $\begin{array}{l}\text { Ramp (Launch) } \\
\text { (RW-080) }\end{array}$ & - & $\begin{array}{l}82.1 \\
(35.4)\end{array}$ & $\begin{array}{l}83.8 \\
(14.0)\end{array}$ & $\begin{array}{l}63.5 \\
(16.6)\end{array}$ & $\begin{array}{l}76.5 \\
(24.5)\end{array}$ \\
\hline 77. & $\begin{array}{l}\text { Mandatory Seat Belt } \\
\text { (R16-1) }\end{array}$ & $\mathrm{ac}$ & $\begin{array}{l}92.8 \\
(32.9)\end{array}$ & $\begin{array}{l}77.4 \\
(13.2)\end{array}$ & $\begin{array}{c}56.8 \\
(14.8)\end{array}$ & $\begin{array}{l}75.7 \\
(25.8)\end{array}$ \\
\hline 78 & $\begin{array}{l}\text { Lighthouse } \\
\text { (RG-120) }\end{array}$ & $\mathrm{ac}$ & $\begin{array}{l}82.6 \\
(24.2)\end{array}$ & $\begin{array}{l}81.4 \\
(22.1)\end{array}$ & $\begin{array}{l}58.1 \\
(12.4)\end{array}$ & $\begin{array}{l}74.0 \\
(22.2)\end{array}$ \\
\hline 791 & $\begin{array}{l}\text { Showers } \\
\text { (RA-130) }\end{array}$ & $\mathrm{ac}$ & $\begin{array}{c}77.2 \\
(20.9)\end{array}$ & $\begin{array}{c}78.6 \\
(13.8)\end{array}$ & $\begin{array}{c}58.1 \\
(12.4)\end{array}$ & $\begin{array}{c}71.3 \\
(18.2)\end{array}$ \\
\hline
\end{tabular}


Table 2. Mean legibility distance (m) and standard deviations (SD) of 85 symbol signs for young, middle-aged, and older drivers (continued).

\begin{tabular}{|c|c|c|c|c|c|c|}
\hline $\begin{array}{l}\text { Rank } \\
\text { (Dist) } \\
\end{array}$ & $\begin{array}{l}\text { Sign Name } \\
\text { (MUTCD No.) }\end{array}$ & $\begin{array}{l}\text { Age } \\
\text { Diff. }\end{array}$ & $\begin{array}{l}\text { Young } \\
\underline{(\mathrm{SD})}\end{array}$ & $\begin{array}{l}\text { Middle- } \\
\underline{\text { Aged }} \\
\underline{(\mathrm{SD})}\end{array}$ & $\frac{\text { Older }}{(\mathrm{SD})}$ & $\begin{array}{l}\text { Mean } \\
\text { Dist } \\
(\mathrm{SD})\end{array}$ \\
\hline 80 . & $\begin{array}{l}\text { Sledding } \\
(\mathrm{RS}-060)\end{array}$ & $\mathrm{a}$ & $\begin{array}{c}82.0 \\
(31.5)\end{array}$ & $\begin{array}{l}70.8 \\
(12.6)\end{array}$ & $\begin{array}{c}57.8 \\
(13.9)\end{array}$ & $\begin{array}{c}70.2 \\
(22.2)\end{array}$ \\
\hline 81. & $\begin{array}{l}\text { Skating (Ice) } \\
\text { (RS-010) }\end{array}$ & $\mathrm{a}$ & $\begin{array}{l}77.4 \\
(24.0)\end{array}$ & $\begin{array}{l}69.6 \\
(13.4)\end{array}$ & $\begin{array}{c}60.3 \\
(11.2)\end{array}$ & $\begin{array}{c}69.1 \\
(17.5)\end{array}$ \\
\hline 82. & $\begin{array}{l}\text { Ranger Station } \\
\text { (RG-170) }\end{array}$ & $c$ & $\begin{array}{c}65.0 \\
(16.8)\end{array}$ & $\begin{array}{c}75.6 \\
(19.6)\end{array}$ & $\begin{array}{l}57.5 \\
(10.7)\end{array}$ & $\begin{array}{c}66.0 \\
(16.8)\end{array}$ \\
\hline 83. & $\begin{array}{l}\text { Emergency Medical } \\
\text { Service } \\
\text { (D9-13) }\end{array}$ & $\mathrm{ac}$ & $\begin{array}{l}68.9 \\
(21.6)\end{array}$ & $\begin{array}{c}67.6 \\
(17.0)\end{array}$ & $\begin{array}{r}52.4 \\
(9.4)\end{array}$ & $\begin{array}{c}63.0 \\
(17.4)\end{array}$ \\
\hline 84. & $\begin{array}{l}\text { Train Station } \\
\text { (I-7) }\end{array}$ & $\mathrm{c}$ & $\begin{array}{c}56.4 \\
(20.3)\end{array}$ & $\begin{array}{l}62.5 \\
(15.2)\end{array}$ & $\begin{array}{l}45.7 \\
(16.7)\end{array}$ & $\begin{array}{c}54.9 \\
(18.5)\end{array}$ \\
\hline 85. & $\begin{array}{l}\text { No Hitchhiking } \\
\text { (R9-4a) }\end{array}$ & $\mathrm{a}$ & $\begin{array}{l}51.6 \\
(22.5)\end{array}$ & $\begin{array}{l}45.2 \\
(10.8)\end{array}$ & $\begin{array}{l}36.5 \\
(7.9)\end{array}$ & $\begin{array}{c}44.4 \\
(15.2)\end{array}$ \\
\hline
\end{tabular}




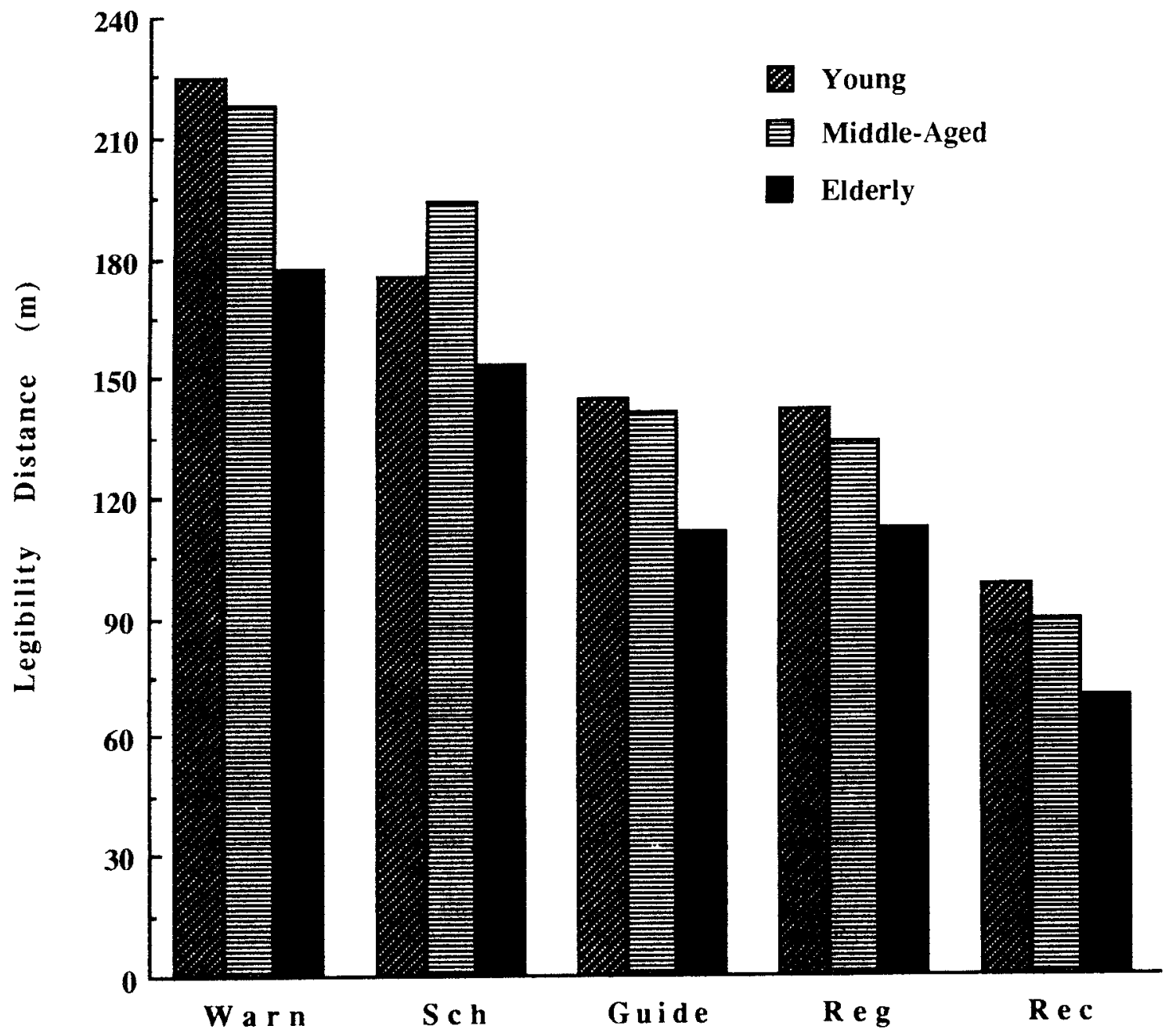

Sign Type

$1 \mathrm{~m}=1.09 \mathrm{yds}$

Figure 2. Daytime legibility distance of different sign types as a function of driver age (phase 1). 
Table 3. Mean legibility distance $(\mathrm{m})$ of different sign types among young, middle-aged, and older drivers.

\begin{tabular}{|c|c|c|c|c|c|}
\hline Sign Type & $\frac{\text { Age }}{\text { Diff }}$ & Young & $\begin{array}{c}\text { Middle- } \\
\text { Aged }\end{array}$ & $\underline{\text { Old }}$ & Mean \\
\hline Warning & $\mathrm{ac}$ & 224.5 & 217.9 & 177.3 & 206.6 \\
\hline School & - & 174.8 & 193.5 & 152.8 & 173.7 \\
\hline Guide & $\mathrm{ac}$ & 144.0 & 140.7 & 111.6 & 132.1 \\
\hline Regulatory & $\mathrm{ac}$ & 141.6 & 133.5 & 112.0 & 129.0 \\
\hline Recreational and Cultural & $\mathrm{ac}$ & 97.9 & 89.2 & 69.8 & 85.6 \\
\hline Mean & & 156.6 & 155.0 & 124.7 & 145.4 \\
\hline
\end{tabular}

Notes:

$\mathrm{a}=$ Significant difference $(\mathrm{p}<.05)$ between Young and Older Drivers

$\mathrm{c}=$ Significant difference $(\mathrm{p}<.05)$ between Middle-Aged and Older Drivers

Table 4. Legibility distance (m) of warning signs among young, middle-aged, and older drivers.

\begin{tabular}{|c|c|c|c|c|c|c|}
\hline $\begin{array}{l}\text { Rank } \\
\text { (Dist) } \\
\end{array}$ & $\begin{array}{l}\text { Sign Name } \\
\text { (MUTCD No.) }\end{array}$ & $\begin{array}{l}\text { Age } \\
\text { Diff }\end{array}$ & Young & $\begin{array}{r}\text { Middle } \\
\text { Aged }\end{array}$ & $\underline{\text { Older }}$ & $\begin{array}{c}\text { Mean } \\
\text { Dist }\end{array}$ \\
\hline 1 & $\begin{array}{l}\text { Cross Road } \\
\text { (W2-1) }\end{array}$ & - & 385.4 & 356.8 & 299.4 & 347.2 \\
\hline 2. & $\begin{array}{l}\text { Added Lane } \\
\text { (W4-3) }\end{array}$ & $\mathrm{a}$ & 363.9 & 343.0 & 267.7 & 324.9 \\
\hline 3. & $\begin{array}{l}\text { Y Symbol } \\
\text { (W2-5) }\end{array}$ & - & 321.0 & 311.5 & 269.8 & 300.8 \\
\hline 4. & $\begin{array}{l}\text { Side Road (90 deg) } \\
\text { (W2-2) }\end{array}$ & - & 328.7 & 305.6 & 263.6 & 299.3 \\
\hline 5. & $\begin{array}{l}\text { Large Arrow } \\
\text { (W1-6) }\end{array}$ & $\mathrm{ac}$ & 307.6 & 322.1 & 247.4 & 292.4 \\
\hline 6. & $\begin{array}{l}\text { T Symbol } \\
\text { (W2-4) }\end{array}$ & - & 310.2 & 300.3 & 258.3 & 289.6 \\
\hline
\end{tabular}

Notes:

$\mathrm{a}=$ Significant difference $(\mathrm{p}<.05)$ between Young and Older Drivers.

$\mathrm{c}=$ Significant difference $(\mathrm{p}<.05)$ between Middle-Aged and Older Drivers. 
Table 4. Legibility distance (m) of warning signs among young, middle-aged, and older drivers (continued).

\begin{tabular}{|c|c|c|c|c|c|c|}
\hline $\begin{array}{l}\text { Rank } \\
\text { (Dist) }\end{array}$ & $\begin{array}{l}\text { Sign Name } \\
\text { (MUTCD No.) }\end{array}$ & $\begin{array}{l}\text { Age } \\
\underline{\text { Diff }}\end{array}$ & Young & $\begin{array}{c}\text { Middle } \\
\text { Aged }\end{array}$ & $\underline{\text { Older }}$ & $\begin{array}{r}\text { Mear } \\
\text { Dist }\end{array}$ \\
\hline 7. & $\begin{array}{l}\text { Right Curve } \\
\text { (W1-2R) }\end{array}$ & - & 270.5 & 310.1 & 269.1 & 283.2 \\
\hline 8 & $\begin{array}{l}\text { Right Turn } \\
\text { (W1-1R) }\end{array}$ & - & 289.7 & 279.3 & 250.3 & 273.1 \\
\hline 9. & $\begin{array}{l}\text { Right Reverse Curve } \\
\text { (W1-4R) }\end{array}$ & - & 284.2 & 266.9 & 216.4 & 255.8 \\
\hline 10 & $\begin{array}{l}\text { Double Head Arrow } \\
\text { (W1-7) }\end{array}$ & $\mathrm{ac}$ & 268.7 & 290.1 & 205.4 & 254.7 \\
\hline 11. & $\begin{array}{l}\text { Merge } \\
(\mathrm{W} 4-1)\end{array}$ & $\mathrm{ac}$ & 276.2 & 263.8 & 196.5 & 245.5 \\
\hline 12. & $\begin{array}{l}\text { Signal Ahead } \\
\text { (W3-3) }\end{array}$ & - & 256.9 & 253.4 & 214.1 & 241.5 \\
\hline 13 & $\begin{array}{l}\text { Side Road (45 deg) } \\
\text { (W2-3) }\end{array}$ & $\mathrm{c}$ & 259.3 & 266.9 & 196.4 & 240.9 \\
\hline 14 & $\begin{array}{l}\text { Right Reverse Turn } \\
\text { (W1-3R) }\end{array}$ & - & 273.5 & 225.0 & 197.7 & 232.1 \\
\hline 15 & $\begin{array}{l}\text { Winding Road Right } \\
\text { (W1-5R) }\end{array}$ & - & 252.0 & 242.9 & 200.3 & 231.7 \\
\hline 16 & $\begin{array}{l}\text { Lane Reduction } \\
\text { Transition } \\
\text { (W4-2) }\end{array}$ & - & 246.4 & 214.9 & 195.4 & 218.9 \\
\hline 17. & $\begin{array}{l}\text { Yield Ahead } \\
\text { (W3-2a) }\end{array}$ & $\mathrm{a}$ & 264.1 & 224.6 & 165.0 & 217.9 \\
\hline 18 & $\begin{array}{l}\text { Divided Highway } \\
\text { Begins } \\
\text { (W6-1) }\end{array}$ & $\mathrm{a}$ & 236.1 & 199.5 & 176.0 & 203.9 \\
\hline 19. & $\begin{array}{l}\text { Two- Way Traffic } \\
\text { (W6-3) }\end{array}$ & - & 208.0 & 217.9 & 180.0 & 202.0 \\
\hline 20 . & $\begin{array}{l}\text { Divided Highway } \\
\text { Ends } \\
\text { (W6-2) }\end{array}$ & - & 228.5 & 196.5 & 177.8 & 200.9 \\
\hline 21 & $\begin{array}{l}\text { School Advance } \\
\text { (S1-1) }\end{array}$ & - & 202.8 & 215.3 & 184.4 & 200.8 \\
\hline 22 & $\begin{array}{l}\text { Advance Flagger } \\
(\mathrm{W} 20-7 \mathrm{a})\end{array}$ & - & 209.2 & 219.7 & 173.0 & 200.6 \\
\hline 23 & $\begin{array}{l}\text { Chevron Alignment } \\
\text { (W1-8) }\end{array}$ & $\mathrm{a}$ & 223.8 & 202.9 & 165.8 & 197.5 \\
\hline
\end{tabular}


Table 4. Legibility distance (m) of warning signs among young, middle-aged, and older drivers (continued).

\begin{tabular}{|c|c|c|c|c|c|c|}
\hline $\begin{array}{l}\text { Rank } \\
\text { (Dist) }\end{array}$ & $\begin{array}{l}\text { Sign Name } \\
\text { (MUTCD No.) }\end{array}$ & $\begin{array}{l}\text { Age } \\
\text { Diff } \\
\end{array}$ & Young & $\begin{array}{l}\text { Middle } \\
\text { Aged }\end{array}$ & $\underline{\text { Older }}$ & $\begin{array}{c}\text { Mean } \\
\text { Dist }\end{array}$ \\
\hline 24. & $\begin{array}{l}\text { Stop Ahead } \\
\text { (W3-1a) }\end{array}$ & $\mathrm{c}$ & 187.4 & 225.8 & 158.8 & 190.6 \\
\hline 25 & $\begin{array}{l}\text { Slippery When Wet } \\
\text { (W8-5) }\end{array}$ & $\mathrm{c}$ & 191.6 & 206.1 & 155.7 & 184.5 \\
\hline 26. & $\begin{array}{l}\text { Deer Crossing } \\
(\mathrm{W} 11-3)\end{array}$ & - & 190.3 & 199.4 & 162.9 & 184.2 \\
\hline 27. & $\begin{array}{l}\text { Worker (Construction) } \\
\text { (W21-1a) }\end{array}$ & $\mathrm{a}$ & 196.1 & 187.1 & 137.4 & 173.5 \\
\hline 28. & $\begin{array}{l}\text { Low Vertical } \\
\text { Clearance } \\
\text { (W12-2) }\end{array}$ & - & 178.6 & 157.5 & 143.8 & 160.0 \\
\hline 29. & $\begin{array}{l}\text { Pedestrian Crossing } \\
\text { (W11a-2) }\end{array}$ & C & 165.3 & 181.0 & 133.1 & 159.8 \\
\hline 30. & $\begin{array}{l}\text { Cattle Crossing } \\
\text { (W11-4) }\end{array}$ & - & 167.5 & 148.5 & 142.9 & 153.0 \\
\hline 31. & $\begin{array}{l}\text { School Crossing } \\
(\mathrm{S} 2-1)\end{array}$ & C & 146.8 & 171.7 & 121.3 & 146.6 \\
\hline 32 & $\begin{array}{l}\text { Bicycle Crossing } \\
\text { (W11-1) }\end{array}$ & $\mathrm{c}$ & 159.4 & 156.5 & 122.5 & 146.1 \\
\hline 33 & $\begin{array}{l}\text { Railroad Advance } \\
\text { Warning } \\
\text { (W10-1) }\end{array}$ & - & 140.6 & 157.9 & 123.4 & 140.6 \\
\hline 34. & $\begin{array}{l}\text { Narrow Bridge } \\
\text { (W5-2a) }\end{array}$ & $\mathrm{ac}$ & 152.5 & 139.9 & 100.1 & 130.8 \\
\hline 35 & $\begin{array}{l}\text { Tractor Crossing } \\
\text { (W11-5) }\end{array}$ & - & 140.2 & 142.8 & 104.3 & 129.1 \\
\hline 36. & $\begin{array}{l}\text { Hill } \\
(\text { W7-1) }\end{array}$ & $\mathrm{ac}$ & 128.8 & 133.6 & 101.7 & 121.4 \\
\hline 37. & $\begin{array}{l}\text { Playground } \\
\text { (W15-1) }\end{array}$ & - & 120.8 & 118.4 & 107.4 & 115.5 \\
\hline 38 & $\begin{array}{l}\text { Double Arrow } \\
\text { (W12-1) }\end{array}$ & - & 124.6 & 115.3 & 103.0 & 114.3 \\
\hline 39 & $\begin{array}{l}\text { Railroad Parallel to } \\
\text { Roadway } \\
\text { (W10-3) }\end{array}$ & $\mathrm{ac}$ & 114.0 & 101.8 & 80.9 & 98.9 \\
\hline 40 & $\begin{array}{l}\text { Pavement Ends } \\
\text { (W8-3a) }\end{array}$ & $\mathrm{a}$ & 109.9 & 96.5 & 75.0 & 93.8 \\
\hline
\end{tabular}


Table 5. Legibility distance $(\mathrm{m})$ of regulatory signs among young, middle-aged, and older drivers.

\begin{tabular}{|c|c|c|c|c|c|c|}
\hline $\begin{array}{l}\text { Rank } \\
\text { (Dist) }\end{array}$ & $\begin{array}{l}\text { Sign Name } \\
\text { (MUTCD No.) }\end{array}$ & $\begin{array}{l}\text { Age } \\
\text { Diff } \\
\end{array}$ & $\underline{\text { Young }}$ & $\underline{\text { Middle }}$ & $\underline{\text { Older }}$ & $\begin{array}{r}\text { Mean } \\
\text { Dist }\end{array}$ \\
\hline 1. & $\begin{array}{l}\text { Straight or Left } \\
\text { (R3-6) }\end{array}$ & - & 254.0 & 247.1 & 248.5 & 249.9 \\
\hline 2 & $\begin{array}{l}\text { Keep Right } \\
\text { (R4-7) }\end{array}$ & $\mathrm{a}$ & 252.3 & 226.4 & 181.1 & 219.9 \\
\hline 3 & $\begin{array}{l}\text { No Right Turn } \\
\text { (R3-1) }\end{array}$ & $\mathrm{a}$ & 173.2 & 150.9 & 132.2 & 152.1 \\
\hline 4 & $\begin{array}{l}\text { No U-Turn } \\
\text { (R3-4) }\end{array}$ & $\mathrm{a}$ & 153.4 & 144.2 & 109.1 & 135.6 \\
\hline 5 & $\begin{array}{l}\text { No Parking } \\
\text { (R8-3a) }\end{array}$ & - & 147.6 & 138.0 & 116.3 & 134.0 \\
\hline 6 & $\begin{array}{l}\text { No Trucks } \\
\text { (R5-2) }\end{array}$ & $\mathrm{c}$ & 113.6 & 126.2 & 90.8 & 110.2 \\
\hline 7. & $\begin{array}{l}\text { National Network } \\
\text { Route } \\
\left(\begin{array}{lll}\text { R } 14 & 4\end{array}\right)\end{array}$ & $\mathrm{a}$ & 114.2 & 110.2 & 91.4 & 105.3 \\
\hline 8. & $\begin{array}{l}\text { National. Network } \\
\text { Route Prohibited } \\
\text { (R14-5) }\end{array}$ & - & 107.3 & 102.2 & 92.8 & 100.8 \\
\hline 9. & $\begin{array}{l}\text { No Bicycles } \\
\text { (R5-6) }\end{array}$ & $\mathrm{ac}$ & 97.8 & 100.7 & 76.8 & 91.8 \\
\hline 10 . & $\begin{array}{l}\text { Mandatory Seat Belt } \\
\text { (R16-1) }\end{array}$ & $\mathrm{ac}$ & 92.8 & 77.4 & 56.8 & 75.7 \\
\hline 11 & $\begin{array}{l}\text { No Hitchhiking } \\
\text { (R9-4a) }\end{array}$ & $\mathrm{a}$ & 51.6 & 45.2 & 36.5 & \\
\hline
\end{tabular}

Notes:

$\mathrm{a}=$ Significant difference $(\mathrm{p}<.05)$ between Young and Older Drivers.

$\mathrm{c}=$ Significant difference $(\mathrm{p}<.05)$ between Middle-Aged and Older Drivers 
Table 6. Legibility distance (m) of guide signs among young, middle-aged, and older drivers.

\begin{tabular}{|c|c|c|c|c|c|c|}
\hline $\begin{array}{l}\text { Rank } \\
\text { (Dist) }\end{array}$ & $\begin{array}{l}\text { Sign Name } \\
\text { (MUTCD No.) }\end{array}$ & $\begin{array}{l}\text { Age } \\
\text { Diff }\end{array}$ & Young & $\begin{array}{l}\text { Middle- } \\
\text { Aged }\end{array}$ & $\underline{\text { Older }}$ & $\begin{array}{r}\text { Mean } \\
\text { Dist }\end{array}$ \\
\hline 1 & $\begin{array}{l}\text { Hospital } \\
\text { (D9-2) }\end{array}$ & $\overline{\mathrm{ac}}$ & 257.0 & 257.7 & $\overline{201.6}$ & $2 \overline{38.8}$ \\
\hline 2 & $\begin{array}{l}\text { Tourist Information } \\
\text { (D9-10) }\end{array}$ & - & 245.1 & 249.6 & 204.8 & 233.2 \\
\hline 3 & Airport & - & 228.2 & 236.2 & 192.9 & 219.1 \\
\hline 4 & $\begin{array}{l}(\mathrm{I}-5) \\
\text { Phone } \\
\text { (D9-1) }\end{array}$ & - & 212.4 & 203.2 & 176.9 & 197.5 \\
\hline 5. & $\begin{array}{l}\text { Camping } \\
\text { (D9-3) }\end{array}$ & - & 215.2 & 206.1 & 167.8 & 196.4 \\
\hline 6 & $\begin{array}{l}\text { Handicapped } \\
\text { (D9-6) }\end{array}$ & $\mathrm{ac}$ & 213.6 & 211.3 & 161.2 & 195.4 \\
\hline 7. & Dock & $\mathrm{a}$ & 186.7 & 167.4 & 150.0 & 168.1 \\
\hline 8. & $\begin{array}{l}(\mathrm{I}-9) \\
\text { Gas } \\
(\mathrm{D} 9-7)\end{array}$ & $\mathrm{a}$ & 169.5 & 140.7 & 102.7 & 137.6 \\
\hline 9. & $\begin{array}{l}\text { Bus Station } \\
\text { (I-6) }\end{array}$ & - & 124.7 & 131.8 & 100.3 & 118.9 \\
\hline 10 . & $\begin{array}{l}\text { Recreational Vehicle } \\
\text { (D9-3a) }\end{array}$ & ac & 131.1 & 127.5 & 95.2 & 117.9 \\
\hline 11. & $\begin{array}{l}\text { Propane } \\
\text { (D9-15) }\end{array}$ & $\mathrm{c}$ & 114.7 & 121.7 & 95.9 & 110.8 \\
\hline 12. & $\begin{array}{l}\text { Lodging } \\
\text { (D9-9) }\end{array}$ & $\mathrm{a}$ & 118.4 & 106.7 & 90.5 & 105.2 \\
\hline 13 & $\begin{array}{l}\text { Hiking Trail } \\
\text { (I-4) }\end{array}$ & $\mathrm{a}$ & 117.8 & 108.9 & 84.1 & 103.6 \\
\hline 14 & $\begin{array}{l}\text { Recreational Vehicle } \\
\text { Sanitary Station } \\
\text { (D9-12) }\end{array}$ & C & 90.7 & 112.6 & 71.4 & 91.6 \\
\hline 15 & $\begin{array}{l}\text { Winter Recreation } \\
(\mathrm{I}-10)\end{array}$ & $\mathrm{a}$ & 116.0 & 86.6 & 68.1 & 90.2 \\
\hline 16 & Library & - & 97.4 & 93.7 & 71.9 & 87.7 \\
\hline 17. & $\begin{array}{l}(\mathrm{I}-8) \\
\text { Food } \\
(\mathrm{D} 9-8)\end{array}$ & - & 89.9 & 89.9 & 79.5 & 86.4 \\
\hline 18. & $\begin{array}{l}\text { Diesel } \\
\text { (D9-11) }\end{array}$ & $\mathrm{c}$ & 86.4 & 91.5 & 65.8 & 81.2 \\
\hline 19. & $\begin{array}{l}\text { Rest Area } \\
\text { (D5-5a) }\end{array}$ & ac & 83.6 & 82.4 & 65.8 & 77.3 \\
\hline
\end{tabular}

Notes:

$\mathrm{a}=$ Significant difference $(\mathrm{p}<.05)$ between Young and Older Drivers.

$\mathrm{c}=$ Significant difference $(\mathrm{p}<.05)$ between Middle-Aged and Older Drivers 
Table 6. Legibility distance (m) of guide signs among young, middle-aged, and older

20 Emergency Medical Service (D9-13)

21. Train Station (I-7) drivers (continued).

$$
\begin{array}{lllll}
\text { ac } & 68.9 & 67.6 & 52.4 & 63.0
\end{array}
$$

$\begin{array}{lllll}\text { C } & 56.4 & 62.5 & 45.7 & 54.9\end{array}$

Table 7. Legibility distance $(\mathrm{m})$ of recreational and cultural signs among young, middleaged, and older drivers.

\begin{tabular}{|c|c|c|c|c|c|c|}
\hline $\begin{array}{l}\text { Rank } \\
\text { (Dist) }\end{array}$ & $\begin{array}{l}\text { Sign Name } \\
\text { (MUTCD No.) }\end{array}$ & $\begin{array}{l}\text { Age } \\
\text { Diff }\end{array}$ & Young & $\underline{\text { Middle }}$ & $\underline{\text { Older }}$ & $\begin{array}{c}\text { Mean } \\
\text { Dist }\end{array}$ \\
\hline 1. & $\begin{array}{l}\text { Swimming } \\
\text { (RW-130) }\end{array}$ & ac & 153.6 & 144.3 & 104.1 & 134.0 \\
\hline 2. & $\begin{array}{l}\text { Restrooms } \\
(\mathrm{RM}-140)\end{array}$ & $\mathrm{a}$ & 123.2 & 106.7 & 90.8 & 106.9 \\
\hline 3 & $\begin{array}{l}\text { Canoeing } \\
\text { (RW-020) }\end{array}$ & $\mathrm{a}$ & 113.6 & 93.8 & 75.5 & 94.3 \\
\hline 4 & $\begin{array}{l}\text { Snowmobiling } \\
(\mathrm{RS}-070)\end{array}$ & $\mathrm{ac}$ & 110.9 & 100.8 & 71.1 & 94.3 \\
\hline 5 & $\begin{array}{l}\text { Campfire } \\
\text { (RA-030) }\end{array}$ & $\mathrm{ac}$ & 98.3 & 101.0 & 79.5 & 92.9 \\
\hline 6 & $\begin{array}{l}\text { Amphitheater } \\
\text { (RL-010) }\end{array}$ & $\mathrm{a}$ & 100.0 & 82.4 & 71.7 & 84.7 \\
\hline 7. & $\begin{array}{l}\text { Shelter (Sleeping) } \\
\text { (RA-110) }\end{array}$ & $\mathrm{a}$ & 106.8 & 70.8 & 58.7 & 78.8 \\
\hline 8. & $\begin{array}{l}\text { Ramp (Launch) } \\
\text { (RW-08) }\end{array}$ & - & 82.1 & 83.8 & 63.5 & 76.5 \\
\hline 9. & $\begin{array}{l}\text { Lighthouse } \\
\text { (RG-120) }\end{array}$ & $\mathrm{ac}$ & 82.6 & 81.4 & 58.1 & 74.0 \\
\hline 10 . & $\begin{array}{l}\text { Showers } \\
\text { (RA-130) }\end{array}$ & $\mathrm{ac}$ & 77.2 & 78.6 & 58.1 & 71.3 \\
\hline 11. & $\begin{array}{l}\text { Sledding } \\
\text { (RS-060) }\end{array}$ & $\mathrm{a}$ & 82.0 & 70.8 & 57.8 & 70.2 \\
\hline 12 & $\begin{array}{l}\text { Skating (Ice) } \\
\text { (RS-010) }\end{array}$ & $\mathrm{a}$ & 77.4 & 69.6 & 60.3 & 69.1 \\
\hline 13 & $\begin{array}{l}\text { Ranger Station } \\
(\mathrm{RG}-170)\end{array}$ & C & 65.1 & 75.6 & 57.5 & 66.1 \\
\hline
\end{tabular}

Notes:

$\mathrm{a}=$ Significant difference $(\mathrm{p}<.05)$ between Young and Older Drivers.

$\mathrm{c}=$ Significant difference $(\mathrm{p}<.05)$ between Middle-Aged and Older Drivers 


\section{Visual Predictors Of Sign Legibility}

Pearson product-moment correlations between acuity and mean legibility distance were determined both across age groups, as well as separately within each age group; probability levels were adjusted using a Bonferroni correction (see table 8). A strong overall correlation was observed between acuity and sign visibility $(\underline{r}=0.78, \underline{p}<0.001)$. The strength of this relationship varied little with sign type (Warning: $\underline{r}=0.76$; Regulatory: $\underline{r}=0.74$; Guide: $\underline{r}=0.77$;

Recreation/Cultural: $\underline{r}=0.78)$. Acuity-was also an excellent predictor of legibility distance within each age group: young drivers $\underline{\mathrm{r}}=0.76(\mathrm{p}<0.01)$; middle-aged drivers $\underline{\mathrm{r}}=0.76,(\mathrm{p}<0.01)$; and older drivers $\underline{r}=0.66$ ( $\mathrm{p}<0.01)$. The high-cutoff spatial frequency was also strongly related to overall legibility distance $(\underline{r}=0.69, p<0.001)$ and consistent across the different sign types (Warning $\underline{r}=0.67$; Regulatory $\underline{r}=0.63$; Guide $\underline{r}=0.67$; Recreation/ Cultural $\underline{r}=0.67$ ). The highcutoff within-age-group correlations, however, varied considerably between age groups; they were significant for young $(\underline{r}=0.63, \underline{p}<0.05)$ and older drivers $(\underline{r}=0.76, \underline{p}<0.001$, ) but not middle-aged ones $(\underline{r}=0.35, \underline{p}=0.27$ ). The acuity and high cutoff measures were also strongly related to one another $(\underline{\mathrm{r}}=0.64, \mathrm{p}<0.001)$. Transformation of their correlations to dependent $t$ 's indicated that the acuity and high cutoff measures did not differ significantly in the strength of their association with legibility distance $(\underline{\mathrm{t}}=0.21, \mathrm{p}>0.8)$. The overall correlation between peak $\log$ contrast sensitivity and legibility distance $(\underline{r}=0.42, p<0.01)$ was significant. The corresponding within-group correlation was significant for the older $(\underline{\mathrm{r}}=.49, \mathrm{p}<0.05)$, but not young $(\underline{r}=0.32, \underline{p}=.31)$ or middle-aged drivers $(\underline{r}=0.30, \underline{p}=.34)$, a pattern that was repeated in the correlations between peak spatial frequency and legibility distance. Across the three age groups, the correlation was .52 ( $\mathrm{p}<0.001$; for the older $\underline{\mathrm{r}}=.61(\mathrm{p}<0.01)$; for the young $\underline{\mathrm{r}}=0.32$ ( $\mathrm{p}=0.32)$, and among the middle-aged $\underline{\mathrm{r}}=.21,(\mathrm{p}=0.61)$. A series of $r$ to $t$ transformations showed that acuity was a better predictor of sign legibility than both peak log sensitivity $(\mathrm{p}<0.01)$ and peak spatial frequency $(\mathrm{p}<0.05)$; high cutoff was superior to peak log sensitivity $(\mathrm{p}<0.05)$ but not to peak spatial frequency as a predictor of legibility distance $(\mathrm{p}>0.10)$.

Table 8. Correlations between visual measures and the daytime legibility of symbol signs among young, middle-aged, and older drivers.

Age Group

Visual Measure

Acuity

High Cutoff

Peak Log Sensitivity

Peak Spatial Frequency

*Significant at $\underline{p}<.05$ or better
Middle-

Young $\underline{\text { Aged }}$ Older All

$\begin{array}{llll}0.76^{*} & 0.76^{*} & 0.66^{*} & 0.78^{*}\end{array}$

$\begin{array}{llll}0.63^{*} & 0.35 & 0.76^{*} & 0.69 *\end{array}$

0.32

0.30

$0.49 *$

$0.42 *$

0.32

0.21

$0.61 *$

$0.52 *$ 


\section{DISCUSSION}

This study shows that there are very dramatic differences in legibility distances of symbol signs. The legibility distance of the best sign (CROSS ROAD) was almost eight times that of the worst sign (NO HITCHHIKING). Presumably, this difference is attributable to the large, simple, and well separated features of which the CROSS ROAD sign is composed. More generally, inspection of table 2 suggests that there is an inverse relationship between the number of small complex featural details on a symbol sign and the distance at which it is legible.

The present study extends the findings of previous research on the adverse effects of driver age on the daytime legibility of signs to most of the symbol signs in the MUTCD. (See references 3, 14, 30, 37, 57, 127, 163.) Although the differences between them were not always statistically significant, the average legibility distances for older drivers were lower than those of their young and middle-aged counterparts on every one of the 85 signs evaluated (see table 2). Given that the visual acuity of the older drivers was lower than the other two age groups, along with the strong relationship between acuity and visibility, this outcome was not too surprising. It should be noted, however, that the acuity of the older drivers in this study [0.98 minarc or approximately 6/6 (20/20)] was quite a bit better than the average for their age group [about 6/9 to $6 / 15(20 / 30)]$, and about twice as good as the 608 (20/40) acuity cutoff used by most licensing agencies. ${ }^{(170,171)}$ Under daytime conditions, legibility distances 30 percent or so lower than those reported here might be expected for the average 70-year-old driver, and for one operating around the 20/40 limit, legibility distances half those reported here would be more likely. During dusk or nighttime driving, conditions which particularly disadvantage older drivers, legibility distances would almost certainly be affected more adversely. ${ }^{(21,161)}$ To be effective, the design model for future sign improvement efforts must be based on drivers and conditions such as these, and not on average drivers or more ideal conditions.

No significant or consistent mean differences occurred between young and middle-aged drivers. In fact, in several instances, the mean legibility distances of the middle-aged drivers exceeded those of younger drivers. This too appears to reflect the excellent acuity [0.68 minarc or about 6/4 (20/14)] of the middle-aged drivers who participated in this study relative to their age group [6/6 (20/20) or so]. ${ }^{(170)}$ For middle-aged drivers with acuity more representative of their age group [about 6/6 (20/20)], and/or who are operating under more adverse viewing conditions, shorter legibility distances should be expected.

The average legibility distances reported in this investigation are quite consistent with those from earlier research examining age differences in the visibility of symbol signs using CRT-established minimum size thresholds. For example, although the mean of the legibility distances established here are somewhat greater than those on three signs (DIVIDED HIGHWAY, WORKER, and HILL) also tested by Kline, Ghali, Kline, and Brown, the difference is wholly consistent with the lower acuity levels of the young, middle-aged, and older participants in the latter study. ${ }^{(19)}$

A study by Zwahlen, Hu, Sunkara, and Duftis in which the legibility distance of 12 symbol highway signs was determined in on-the-road conditions, has questioned the validity of legibility distances established by laboratory research studies. ${ }^{(172)}$ The thresholds that they 
reported were about double those found in previous research, an outcome that they attributed, with little accompanying justification, to a range of possible factors, including deficiencies of luminance and contrast, or the use of monochrome monitors in laboratory settings. For several reasons, however, their argument is an implausible one. In one of the studies referred to, the investigators demonstrated that their monitor supported target sizes below .50 minarc, the practical human limit on acuity for any luminance/contrast level. ${ }^{(19)}$ Further, and as noted above, Kline et al.'s legibility distances agree well with those in this study even though the former used a monochrome monitor and this study a color one. Additionally, the luminance and contrast levels in previous laboratory work have typically been near or even above the asymptotic level for elevations in acuity. Indeed, it is almost undoubtedly the case that the very high legibility distances found by Zwahlen et al. reflects a very serious methodological flaw in their study, and that is, that their participants were familiarized with each of the 12 signs before the beginning of the experiment. They were then requested to notify the experimenter when they first "recognized" the symbol on the signs they were driving toward. This is wholly unrealistic with regard to typical "real-world" driving where the sign set is not so limited, nor is it known beforehand. More importantly, it also allows the driver to discriminate signs based on justlearned global characteristics as color, shape, layout, and/or a single large feature on the sign, even when they can't see the actual symbol details on it. In other words, once drivers can identify any characteristic that distinguishes a sign from the other 11 in the test set, they can identify it whether or not they can actually discriminate any of its critical features. Obviously, that is a very much easier task than the one typically faced by drivers in the real world, or by observers in the laboratory where they must describe all of its critical features. Although such a "scoring key" approach will decrease the apparent legibility distance of a sign, it provides a much more realistic estimate of the legibility of both unexpected and unfamiliar signs, whether in the laboratory or "out in the real world."

The findings of the present study in regard to age effects on contrast sensitivity were consistent with those from of other studies that have used the VCTS chart, as well as those employing more rigorous psycho-physical procedures. (See references 114, 123, 125, 173.) As spatial frequency was increased, the CSF's for all three age groups rose from the lowest spatial frequencies to a peak at intermediate levels and then declined again. As can be seen in figure 1, the CSF's of all three age groups were within the population norms established for the VCTS. There were significant declines among the older in the spatial frequency at which peak sensitivity occurred, as well as in the high cutoff spatial frequency. And lastly, although it did not reach significance, the magnitude of the age difference in sensitivity was least at low spatial frequencies and increased at intermediate and high spatial frequencies. Two reasons can be offered to account for the absence of the typical significant age by spatial frequency interaction. First, the visually-select character of our observers probably reduced mean sensitivity differences between the age groups. Secondly, to facilitate its speed and ease of administration, the VCTS determines grating thresholds over only eight levels of contrast, and subjects have to guess a grating's orientation from one of only three alternatives. This lack of measurement precision introduces considerable random variation into the data, making it unlikely to see significant age effects unless there are very large mean differences and/or very large samples are tested.

Of the visual measures available within the present study, acuity was the most consistently related to the legibility distance of highway signs. Although not significantly different from the high cutoff CSF measure when compared directly, acuity was more strongly 
related to legibility distance than were peak log sensitivity or peak spatial frequency; high cutoff was superior only to peak log sensitivity. This is somewhat at variance with the findings of both Schieber, Ghali, and Kline, who reported that the high cutoff measure was more strongly associated with legibility distance than was acuity, and Kline and Fuchs, who found that acuity was a relatively poor predictor within age groups. ${ }^{(57,60)}$ Although the reasons for this disparity are not yet clear, it could be that the small sample of signs utilized in the latter two studies were easier to identify based on their low spatial frequency information than was the average sign in the present investigation. Relatedly, the scoring criteria used in those studies may have been less likely to emphasize the high spatial frequency details in the signs tested. Finally, the Schieber et al. study used a much more precise measure of CSF than was utilized here. ${ }^{(60)}$

Had it been available, bandwidth, a more broad-based composite measure from the CSF, might also have proven a useful visibility predictor. Although the limitations of the VCTS at low spatial frequencies (see preceding section) precluded the availability of the bandwidth measure, it is not likely, however, that it would have proven a more effective predictor of visibility than acuity. Based on the assumption that the significant components in regulation signs, even the smaller ones, were placed there by their designers to convey the sign's meaning, a correct response to each sign demands that the observer be able to describe all of its critical details, a task that is essentially an acuity one. In situations where signs are difficult to see for reasons other than size or distance, such as when they are presented in conditions of low contrast (e.g., dusk, or glare), are in motion relative to the observer, or are briefly presented, composite measures derived from the CSF, such as low cutoff, bandwidth, and high cutoff, might more effectively predict driver performance with symbol signs than would acuity. This possibility was one of several evaluated within the context of the night visibility, night visibility with glare, glance legibility, and conspicuity studies carried out as part of this research program. 


\section{CHAPTER 4: COMPREHENSION OF STANDARD SYMBOL SIGNS}

Previous research on the understanding of traffic sign symbols has generally indicated a wide range of comprehension across symbols used on United States roads. Some symbols are clearly understood by 90 percent or more of drivers, while others are understood by fewer than half. The details of past, work on this topic have been presented in the literature review. The little research that has examined the effects of driver age on symbol comprehension indicates that drivers over the age of 65 tend to have more difficulty understanding traffic sign symbols, as compared with young and middle-aged drivers. The reasons for these differences have never been clearly identified, but no doubt relate to older people having learned to drive and done much of their driving when few symbols were used. In addition, the introduction of new symbols into the system is typically done without properly informing those who already have drivers licenses. There is clearly a need to determine how well drivers understand the symbols used on today's roads in order to know where best to concentrate efforts to improve them.

\section{FOCUS GROUP STUDY}

In order to identify the difficulties encountered by older drivers with traffic signs in general, and with symbol signs in particular, the views of these drivers were solicited.

Two focus group sessions with older drivers were held in each of three locations: Calgary, AB; Boise, ID; and San Antonio, TX, in order to gather preliminary information on the concerns older drivers had about traffic signs. The objective of the focus groups was to gather general impressions about the effectiveness of symbol highway signs, to determine what difficulties they present for older drivers, and to explore their advantages and disadvantages, as well as how they might be improved.

\section{METHOD}

Subjects

Forty-nine volunteer subjects, aged 65 or older, participated in the focus groups. All had valid drivers licenses and were active drivers. Education level of the subjects was well spread, with 14 having completed high school graduation, 12 having some University or College education, and 11 having complete University or more. Most subjects primarily drove passenger cars, and the typical annual distance driven was 8000 to $16000 \mathrm{~km}(5,000$ to 9,999 mi). As expected, the participants were very experienced, with the average length of driving being 48.7 years, and the average duration of holding a license being 47.5 years. Nine of the subjects had had some professional driving experience. Slightly fewer than half of the subjects reported doing more than 10 percent of their driving at night, and only five reported doing more than 30 percent of their driving at night. The driving environments were about evenly split between inner city and suburbs of a city.

\section{Procedure}

At the beginning of each session the researchers introduced themselves and the subjects in turn introduced themselves to the group. The objective of the study was explained and subjects were told what was expected of them. It was indicated that their views on traffic signs 
were an important contribution, that they were the only ones who could relate to us the experiences of older drivers. Subjects completed a brief demographic questionnaire (see appendix C) before the session began. The concept of a symbol was explained to the group and examples were given. Then subjects were shown a set of 12 symbol signs from the MUTCD and asked to write out their meanings on an answer sheet, in an effort to stimulate discussion. Some symbols were quite obvious to most subjects, while one or two were confusing or unknown.

\section{RESULTS}

The discussion centered around a list of questions used to guide the group's input. These questions were asked, if the issues did not come up in spontaneous discussion, in approximately the order they appear below. The following is a summary of the main comments and observations offered by the subjects.

1. Do you have trouble seeing or noticing traffic signs?

Signs are often missed or difficult to detect because they are: obscured by trees or vegetation; too small (e.g., street name signs); placed inappropriately (e.g., not far enough in advance), or placed inconsistently from one location to another.

2. Are you able to see and understand traffic sign symbols far enough away to respond to them while driving?

Signs need to be placed farther in advance of the location where their message is required. Symbols are easier to see at a distance than are word signs.

3. Is it easier for you to get information from word signs or symbol ones?

Subjects consistently indicated that symbol signs were better than words for quick recognition, legibility distance, and speed of interpretation.

4. Are symbol signs easier or more difficult to notice on the roadway?

Only one subject suggested that symbol signs were easier to notice, but it was indicated again that symbol signs are "a quicker read" than word signs.

5. Are symbol signs better or worse than word signs when it is dark, raining, or snowing? It was generally felt that there was no difference in conspicuity under adverse environmental conditions. It was pointed out that sign reflectivity helped a lot at night.

6. Are symbol signs more or less easy to make out or "read" than word signs from a distance?

There was considerable agreement that symbol signs could be read at a greater distance than words.

7. Are symbol signs better or worse when there is a lot of visual clutter (e.g., other traffic signs, advertising signs, buildings) in the area?

Some subjects indicated that symbol signs would be easier to detect under these conditions, but it was the general view that visual clutter and distraction from advertising is a significant problem when trying to find any sign information. 
8. Are you able to understand the meanings of symbols? (Which ones give you trouble? Which ones are the best?)

There was a consistent view that symbol signs are better for most messages. Words are better for some, and words along with the symbols may be necessary to help drivers learn the meanings. It was pointed out that familiarity is an important factor in understanding a symbol (some signs are never seen by some drivers) and that the roadway context helps understand some symbols. Symbols are also better because they can be understood by foreigners.

9. What steps, if any, do you take to help you find and identify traffic signs at night, in the rain, or fog?

The main approaches mentioned were: drive less at night; drive more slowly at night;

drive in familiar areas only; get assistance from passenger; dim the dash lights; have a clean windshield; and squint to see signs better.

10. Do you rely on a passenger to read signs and help with the driving in this way? The major use of a passenger was in unfamiliar areas.

11. Have you ever been involved in a traffic accident in which signs were a factor? Only one subject indicated involvement in an accident where a traffic sign might have been a factor, and this was a case where poor placement led another driver to run a "stop" sign.

12. What can be done to improve traffic sign symbols?

A number of suggestions were made for the improvement of symbols: bolder symbols, fewer small details, increase sign size, consistency in design across the country, design with more realism (e.g., more realistic looking people), use only symbols that are readily recognized, indicate that the "hill" sign is for trucks only.

The following general comments are also worthy of note:

- Symbol signage tends to be overused.

- We need more permissive and few regulatory signs.

- Use bigger, brighter "stop" signs.

- $\quad$ Try out new sign designs on drivers before using then

- $\quad$ Issue pamphlets describing new signs when renewing licenses.

- $\quad$ Some old drivers drive too slowly and too cautiously.

- Adverse weather conditions affect visibility.

\section{DISCUSSION}

In general, the older drivers sampled felt that symbol signs had advantages over word signs, and few specific complaints were made. The major difficulties presented by traffic signs were problems in identifying some symbols at a distance due to small details in the symbol, inability to detect signs due to visual clutter, and inadequate time to take in and process sign information, due to signs being poorly placed. 
The literature outlined earlier indicates that older drivers have significant limitations in acquiring and using information from traffic sign symbols. The design of traffic signs must take into account the decrements that older drivers experience in sensory, perceptual, cognitive, and psychomotor function. Information provided by participants in the focus groups has also identified some of the problems with traffic signs, and has provided suggestions for their improvement. Signs are difficult for older drivers to notice, especially at night and under conditions of high information load. Older drivers feel they would benefit from warning and guide signs placed farther in advance of hazards and choice points, from bigger and brighter signs, and from symbols that have fewer small details.

\section{COMPREHENSION STUDY}

The objective of this study was to determine the level of comprehension, or understanding, of the set of FHWA approved 85 traffic sign symbols. The influence of age on comprehension was the variable of central interest.

\section{METHOD}

\section{Subjects}

The subjects in this study were volunteer licensed drivers, aged 18 and older, in Texas, Idaho, and Alberta, Canada. The numbers of drivers are indicated by age group, sex, and location in table 9. They were solicited in several ways: from existing subject pools in Texas and Alberta; driver licensing offices; local service clubs; recreational/social organizations for senior citizens; newspaper ads; as well as from personal contact by the experimenters. Subjects received an honorarium of $\$ 25.00$ for their participation.

Table 9. Number of subjects as a function of location and age: comprehension study (phase 1).

\begin{tabular}{|c|c|c|c|c|c|c|c|}
\hline \multirow[t]{2}{*}{$\underline{\text { Age }}$} & \multicolumn{2}{|c|}{$\underline{\text { Texas }}$} & \multicolumn{2}{|c|}{ Idaho } & \multicolumn{2}{|c|}{ Alberta } & Totals \\
\hline & Male & Female & $\underline{\text { Male }}$ & Female & Male & Female & \\
\hline 18 to 39 & 27 & 24 & 26 & 26 & 27 & 31 & 161 \\
\hline 40 to 59 & 24 & 25 & 26 & 26 & 31 & 28 & 160 \\
\hline 60 to 69 & 11 & 12 & 13 & 15 & 14 & 15 & 80 \\
\hline $70+$ & 14 & 11 & 13 & 11 & 16 & 14 & 79 \\
\hline Totals & 76 & 72 & 78 & 78 & 88 & 88 & 480 \\
\hline$\underline{\text { Site }}$ & \multicolumn{2}{|c|}{148} & \multicolumn{2}{|c|}{156} & \multicolumn{2}{|c|}{176} & \\
\hline
\end{tabular}


Demographic data are shown in table 10. It can be seen that middle-aged and younger subjects drove greater distances over the previous 12 months and drove more at night. The older groups also drove slightly more in the inner city. The passenger car was the most frequently driven vehicle for all age groups.

\section{Stimuli And Apparatus}

The stimuli were 86 color slides of traffic sign symbols from the 1988 MUTCD. Each sign essentially filled the slide. Stimuli were generated by computer, as described in chapter 3 . The signs represented seven categories of sign message: regulatory, warning, guidance, recreation, school zones, construction zones, and railroad crossings.

A Kodak carousal slide projector (Model 4000) was used to project slides of the traffic signs. The screens used for the display of the signs varied in size, depending on the dimensions of the test room used. Test booklets consisting of 19 pages were used for subjects to provide the background information requested, and to write their responses.

\section{Procedure}

The major variable of interest was driver age. Approximately equal numbers of drivers were tested in each age $\mathrm{X}$ gender $\mathrm{X}$ location combination. The age categories were 18 to 39,40 to 59, 60 and above (this last category was divided into those in their 60's and those 70 and above, to ensure a good representation of older drivers).

Subjects were tested in groups ranging in size up to 40. Age and gender were intermixed in most test groups, except where testing was done at organizations for senior citizens, where all were in the $60+$ category. Test facilities ranged from classrooms large enough for 20 to an auditorium that seated 90 . All facilities had chairs and tables and adjustable lighting, permitting levels of illumination appropriate for viewing slides clearly, while at the same time writing answers in the booklet. Subjects sat at distances from the screen from 3 to $12 \mathrm{~m}$ (10 to $40 \mathrm{ft}$ ). In all cases, the sizes of the signs projected onto the screen were large enough to be seen easily by all subjects from the maximum distance.

At the beginning of the session, participants were explained the background of the study and its purpose-to assess the understandability of traffic sign symbols in an effort to develop ways of improving them in the future. The basic procedure to be followed was outlined verbally and subjects were instructed to read and sign the informed consent form, to complete the twopage driver experience questionnaire, to complete the first page of the answer booklet and read the instructions describing the procedure. Following this, the experimenter answered all questions posed by the participants. The subjects' task was to write in the appropriate place in the answer booklet the meaning of each sign, then immediately after giving the answer to indicate, on a 5-point rating scale beneath their answer, how familiar they were with that symbol. Each session began by having subjects indicate the meaning of, and their familiarity, with a practice sign (NO PEDESTRIANS: R9-3a). Subjects were asked again whether there were any questions about the procedure, and further questions were answered before proceeding. The signs were presented in one of six previously determined random orders. Halfway through presentation of the slides, subjects were given a 15-min break. Following completion of the testing, subjects were paid and thanked for their participation. 
Table 10. Participant demographics-comprehension study (phase 1)

(Percentage of Drivers).

Age Group

Distance Driven

$\begin{array}{ccc}\text { Young } & \begin{array}{r}\text { Age Group } \\ \text { Middle-aged }\end{array} & \\ & & \\ 11 & 6 & 24 \\ 14 & 18 & 40 \\ 22 & 19 & 73 \\ 22 & 25 & 41 \\ 15 & 16 & 19 \\ 16 & 16 & 3\end{array}$

Driving Environment

rural/sparsely populated

8

small town

suburbs of city

55

inner city

35

$\begin{array}{rr}6 & 15 \\ 3 & 4 \\ 53 & 94 \\ 38 & 87\end{array}$

Type of Vehicle

passenger car

84

motor home/RV

0

motorcycle

1

light truck/van

heavy truck

14

bus

$\mathrm{cab}$

$73 \quad 67$

$0 \quad 0$

$0 \quad 0$

Percentage Driven

\section{at Night}

23

32

1

0

0

0

0

3

1

0

$0-5$

6-10

11-15

16-20

21-30

$31-40$

\section{5}

8

13

19

20

15

41-50

51 or more
17

3

4

3

5

67
0
0
2
0
1
0




\section{RESULTS}

The answers were scored as correct (2), partially correct (1), or incorrect (0). The response was considered to be wrong when no answer was given. The criteria used for scoring are shown in appendix E. Prior to finalizing the scoring criteria, data from 60 subjects, representing all age $\mathrm{X}$ gender $\mathrm{X}$ location combinations, were scored by both the principal investigator and a research assistant to determine reliability of the scoring. Inter judge agreement on the scoring was found to be very good at 95 percent. Thereafter, the research assistant scored all comprehension data.

The frequency of each response made to each sign, as a function of age and gender can be seen in table 11 . The mean percent correct and mean familiarity ratings are given in table 12 . On the one hand, it can be seen that many symbols are very well understood, with comprehension being over 90 percent for 28 signs. On the other hand, 12 were understood by fewer than 50 percent of the subjects.

Comprehension was examined for each of the sign categories separately, to determine whether drivers were more knowledgeable about some types of signs than others. It can be seen from the data in table 13 that regulatory and railroad crossing signs were understood best, while recreation messages were least understood. Figure 3 shows how comprehension of the different sign types differed across the three age groups.

A significant $(\mathrm{p}<.01)$ correlation between familiarity and comprehension, which occurred for 57 of the signs, is what might be expected-familiar signs are better understood. However, some unfamiliar ones (e.g., playground for Canadian Ss) were well understood, suggesting they were well designed for ease of comprehension.

The version of the Rest Area message tested on the day legibility measure was the one in the 1988 MUTCD, a picnic table beside a leaning tree (D5-5a). The sign prepared for comprehension testing (based on the version in Standard Highway Signs) was a large picnic table, without a tree. That version was tested for comprehension at all three locations. However, when this deviation was discovered (after comprehension testing had begun), it was decided to test both versions in the comprehension measure with the Alberta sample. From table 12, it can be seen that the version without the tree (sign 48) was slightly more easy to understand. It seems likely that its legibility distance would be greater than that for D5-5a, as the latter has considerably more small detail.

The relative level of understanding for each sign was determined by rank ordering the number of correct and partially correct (1 and 2) scores combined for each sign. The rationale for this decision was that many of the partially correct responses did indicate some, but not complete, understanding of the symbol, hence it was considered appropriate to gauge understandability on the basis of both types of responses.

\section{DISCUSSION}

The results of the comprehension study confirm and extend the findings of other researchers, who have reported lower levels of comprehension of traffic sign symbols among older drivers. Symbols which are seen on a regular basis on the roadway, such as those on m any of the regulatory and warning signs are often well understood. Ratings of familiarity relate 
positively to the level of understanding, as would be expected. However, it is evident that many symbols on today's highways are poorly understood by drivers, especially older ones. Results of this comprehension study, including the types of errors made, and comments from participants, have provided some insight into possible improvements that could be incorporated into the redesign of existing signs, and the development of future symbols.

There are various methods for determining the level of comprehension of symbols. All have their advantages and disadvantages. One disadvantage of the technique used here is that some subjects (especially older drivers) have trouble thinking of the appropriate word, or how to express what they want to say. The criteria for scoring are also a factor, since the criteria for what constitutes a correct, partially correct, and incorrect score are somewhat arbitrary. For example, a response to the symbol for straight or left (lane control) was considered wrong if there was no indication that the message referred to a specific lane. In general, any response which clearly indicated that the subject had a sufficient understanding to be able to make a correct response (e.g., to watch for hazards such as a curve in the road or construction ahead), or decision (e.g., take the next left turn to reach the bus station), was scored as correct. In spite of this, levels of comprehension for some symbols may have been underestimated, as the signs were not seen in context, which often provides information that aids in understanding a sign message. An example is the ADVANCE FLAGGER sign that would be seen as one approaches a construction site where it would not likely be interpreted as school crossing guard. Context may also play a role in understanding of curve and turn signs, but this advantage would be somewhat reduced under night driving conditions. Confusion among certain symbols (SCHOOL CROSSING vs. SCHOOL ADVANCE) may not be serious, especially if the advance is thought to be the crossing, as drivers would be alerted to slow and watch for children in both cases.

Table 11. Number of responses to each symbol as a function of age and gender.

\begin{tabular}{|c|c|c|c|c|c|c|c|c|c|}
\hline \multicolumn{3}{|c|}{ SIGN NAME and MUTCD\# } & $\underline{\mathrm{AGE}}$ & \multicolumn{3}{|c|}{$\underline{\text { MALES }}$} & \multicolumn{3}{|c|}{$\underline{\text { FEMALES }}$} \\
\hline \multicolumn{3}{|c|}{ Response } & & 0 & 1 & 2 & $\mathbf{0}$ & 1 & 2 \\
\hline \multirow[t]{4}{*}{1.} & Right & R3-1 & Y & 1 & 0 & 79 & 1 & $\mathbf{0}$ & 80 \\
\hline & Turn & & M & 0 & 1 & 80 & 3 & 1 & 74 \\
\hline & & & 0 & 2 & 0 & 36 & 2 & 0 & 40 \\
\hline & & & $\mathrm{E}$ & 1 & 0 & 42 & 5 & 0 & 31 \\
\hline \multirow[t]{4}{*}{2 . } & No U Turn & R 3-4 & Y & 1 & 0 & 79 & 1 & 0 & 80 \\
\hline & & & M & 0 & 0 & 81 & 2 & 1 & 75 \\
\hline & & & 0 & 2 & 0 & 36 & 0 & 2 & 40 \\
\hline & & & $\mathrm{E}$ & 2 & 0 & 41 & 3 & 0 & 33 \\
\hline \multirow[t]{4}{*}{3.} & Straight & R3-6 & Y & 66 & 8 & 6 & 59 & 3 & 19 \\
\hline & Or Left & & M & 76 & 2 & 3 & 67 & 1 & 10 \\
\hline & (Lane Control) & & 0 & 32 & 3 & 3 & 31 & 4 & \\
\hline & & & E & 38 & 2 & 3 & 31 & 3 & \\
\hline
\end{tabular}

Response: $0=$ incorrect; $1=$ partially correct; $2=$ correct Age: $\mathrm{Y}=18$ to $39 ; \mathrm{M}=40$ to $59 ; 0=60$ to $69 ; \mathrm{E}=70+$

* Signs for which subjects 60 and older had lower comprehension than subjects under 60 
Table 11. Number of responses to each symbol as a function of age and gender (continued).

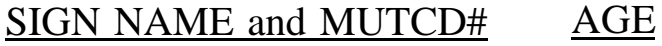
Response

4. Keep Right * R4-7

5. No Trucks R5-2

6. No Bicycles R5-6

7. No Parking R8-3a

8. No

Hitchhiking

9. National

Network

Route

IO. National

Network

Prohibited

11. Mandatory R16-1

Seat Belt*

R14-4

R9-4a

R14-5

R16-1

12. Right Turn

W1-1R

13. Right Curve $* \mathrm{~W} 1=2 \mathrm{R}$
MALES

$\mathrm{Y}$

0

E

Y

$\mathrm{M}$

0

E

$\begin{array}{llll}\mathrm{Y} & 0 & 0 & 80\end{array}$

$\begin{array}{llll}\text { M } & 1 & 2 & 78\end{array}$

0

E

$\mathrm{Y}$

$\mathrm{M}$

0

E

$\mathrm{Y}$

M

0

E

$\mathrm{Y}$

$\mathrm{Y}$
$\mathrm{M}$

E

E

$\mathrm{Y}$

$\mathrm{M}$

0

E

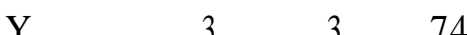

$\mathrm{M}$

0

E

$\mathrm{Y}$
$\mathrm{M}$

E

Y

$\mathrm{M}$

0

E
FEMALES

$\begin{array}{rrr}0 & 1 & 2 \\ 10 & 40 & 31 \\ 13 & 25 & 40 \\ 9 & 12 & 21 \\ 12 & 8 & 16 \\ 2 & 0 & 79 \\ 1 & 2 & 75 \\ 0 & 5 & 37 \\ 3 & 1 & 32\end{array}$

$\begin{array}{lll}1 & 0 & 80\end{array}$

$\begin{array}{lll}3 & 1 & 74\end{array}$

1275

$1 \quad 3 \quad 71$

$\begin{array}{lll}2 & 0 & 79\end{array}$

$\begin{array}{lll}3 & 0 & 75\end{array}$

$3 \quad 0 \quad 39$

$\begin{array}{lll}5 & 0 & 31\end{array}$

$\begin{array}{lll}1 & 0 & 80\end{array}$

$\begin{array}{lll}4 & 0 & 74\end{array}$

$1 \quad 0 \quad 41$

$\begin{array}{lll}6 & 3 & 27\end{array}$

$\begin{array}{lll}38 & 18 & 25\end{array}$

$30 \quad 24 \quad 24$

$16 \quad 15 \quad 11$

$\begin{array}{lll}23 & 9 & 4\end{array}$

$\begin{array}{lll}20 & 27 & 34\end{array}$

$\begin{array}{lll}17 & 33 & 28\end{array}$

$\begin{array}{lll}9 & 17 & 16\end{array}$

$14 \quad 16 \quad 6$

$\begin{array}{lll}4 & 4 & 73\end{array}$

$15 \quad 8 \quad 55$

$21 \quad 1 \quad 20$

$24 \quad 0 \quad 12$

$\begin{array}{lll}9 & 19 & 53\end{array}$

$\begin{array}{lll}10 & 11 & 57\end{array}$

$\begin{array}{lll}2 & 2 & 38\end{array}$

$\begin{array}{lll}6 & 6 & 24\end{array}$

$\begin{array}{lll}2 & 27 & 52\end{array}$

$\begin{array}{lll}2 & 27 & 49\end{array}$

$\begin{array}{lll}5 & 10 & 27\end{array}$

$3 \quad 16 \quad 17$ 
Table 11. Number of responses to each symbol as a function of age and gender (continued).

\begin{tabular}{|c|c|c|c|c|c|c|c|c|}
\hline \multirow{2}{*}{\multicolumn{2}{|c|}{$\frac{\text { SIGN NAME and MUTCD\# }}{\text { Response }}$}} & \multirow[t]{2}{*}{$\underline{\mathrm{AGE}}$} & \multicolumn{3}{|c|}{$\underline{\text { MALES }}$} & \multicolumn{3}{|c|}{ FEMALES } \\
\hline & & & 0 & 1 & 2 & 0 & 1 & 2 \\
\hline 14. Right & W1-3R & $\mathrm{Y}$ & 16 & 35 & 29 & 20 & 35 & 26 \\
\hline Reverse & & M & 25 & 31 & 25 & 26 & 32 & 20 \\
\hline Turn & & 0 & 11 & 14 & 13 & 12 & 12 & 18 \\
\hline & & E & 15 & 14 & 14 & 17 & 14 & 5 \\
\hline 15. Right & W1-4R & $\mathrm{Y}$ & 19 & 45 & 16 & 23 & 47 & 11 \\
\hline Reverse & & M & 19 & 39 & 23 & 27 & 40 & 11 \\
\hline Curve & & 0 & 12 & 13 & 13 & 16 & 20 & 6 \\
\hline & & $\mathrm{E}$ & 13 & 22 & 8 & 19 & 13 & 4 \\
\hline 16. Winding & W1-5R & $\mathrm{Y}$ & 4 & 6 & 70 & 3 & 10 & 68 \\
\hline Road (Right) & & M & 5 & 10 & 66 & 7 & 11 & 60 \\
\hline & & 0 & 4 & 5 & 29 & 1 & 6 & 35 \\
\hline & & $\mathrm{E}$ & 1 & 8 & 34 & 5 & 5 & 26 \\
\hline 17. Large & W1-6 & $\mathrm{Y}$ & 50 & 12 & 18 & 54 & 13 & 14 \\
\hline Arrow & & M & 49 & 13 & 19 & 45 & 16 & 17 \\
\hline & & 0 & 24 & 9 & 5 & 22 & 11 & 9 \\
\hline & & $\mathrm{E}$ & 27 & 10 & 6 & 22 & 5 & 9 \\
\hline 18. Double & W1-7 & $\mathrm{Y}$ & 17 & 16 & 47 & 13 & 24 & 44 \\
\hline Head & & M & 29 & 14 & 38 & 24 & 23 & 31 \\
\hline Arrow * & & 0 & 16 & 9 & 13 & 17 & 8 & 17 \\
\hline & & $\mathrm{E}$ & 21 & 11 & 11 & 19 & 10 & 7 \\
\hline \multirow{4}{*}{$\begin{array}{l}\text { 19. Chevron } \\
\text { Alignment }\end{array}$} & W1-8 & $\mathrm{Y}$ & 41 & 25 & 14 & 40 & 24 & 17 \\
\hline & & M & 47 & 21 & 13 & 61 & 13 & 4 \\
\hline & & 0 & 25 & 8 & 5 & 34 & 7 & 1 \\
\hline & & $\mathrm{E}$ & 37 & 2 & 4 & 24 & 10 & 2 \\
\hline \multirow{4}{*}{ 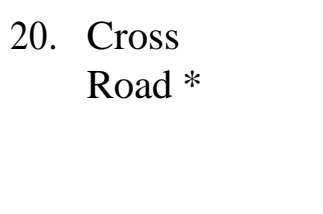 } & W2-1 & $\mathrm{Y}$ & 1 & 0 & 79 & 2 & 0 & 79 \\
\hline & & M & 2 & 2 & 77 & 6 & 1 & 71 \\
\hline & & 0 & 4 & 0 & 34 & 8 & 2 & 32 \\
\hline & & $\mathrm{E}$ & 6 & 0 & 37 & 9 & 1 & 26 \\
\hline \multirow{4}{*}{$\begin{array}{l}\text { 21. Side Road } \\
\text { (Right, } \\
90 \text { Deg) }\end{array}$} & W2-2 & $\mathrm{Y}$ & 9 & 20 & 51 & 8 & 24 & 49 \\
\hline & & M & 3 & 18 & 60 & 16 & 14 & 48 \\
\hline & & 0 & 6 & 9 & 23 & 6 & 7 & 29 \\
\hline & & $\mathrm{E}$ & 7 & 11 & 25 & 9 & 12 & 14 \\
\hline \multirow{4}{*}{$\begin{array}{l}\text { 22. Side Road } \\
\text { (Right, } \\
45 \text { Deg) }\end{array}$} & W2-3 & $\mathrm{Y}$ & 28 & 27 & 25 & 18 & 42 & 21 \\
\hline & & M & 15 & 40 & 26 & 26 & 38 & 14 \\
\hline & & 0 & 6 & 19 & 13 & 10 & 27 & 5 \\
\hline & & $\mathrm{E}$ & 15 & 16 & 12 & 14 & 18 & 4 \\
\hline \multirow[t]{4}{*}{ 23. T Symbol * } & W2-4 & $\mathrm{Y}$ & 10 & 15 & 55 & 9 & 7 & 65 \\
\hline & & M & 13 & 9 & 59 & 19 & 16 & 43 \\
\hline & & 0 & 11 & 5 & 22 & 18 & 6 & 18 \\
\hline & & $\mathrm{E}$ & 11 & 12 & 20 & 14 & 9 & 13 \\
\hline
\end{tabular}


Table 11. Number of responses to each symbol as a function of age and gender (continued).

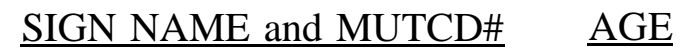
Response

24. Y Symbol

W2-5

26. Yield Ahead * W3-2a

25. Stop Ahead * W3-la

27. Signal Ahead *

28. Merge *

W4-1

W3-3

W4-1

29. Added Lane* W4-3

30. Lane

Reduction

Transition

31. Narrow

Bridge

W5-2a

W4-2

W5-2a

32. Divided W6-1

Highway *

33. Divided

Highway

Ends *
MALES

$0 \quad 12$

$\begin{array}{lll}8 & 12 & 60\end{array}$

Y

M

0

E

Y

M

0

$\mathrm{E}$

$\mathrm{Y}$
$\mathrm{M}$

0

E

Y

$\mathrm{M}$

0

E

$\mathrm{Y}$
$\mathrm{M}$

0

E

Y

$\mathrm{M}$

0

E

Y

Y

M

0

E

Y

M

$$
0
$$

(

E

Y

M

0

E

Y

M

0

E

\begin{tabular}{rrr} 
& \multicolumn{2}{l}{ FEMALES } \\
\cline { 2 - 3 } & 1 & 2 \\
6 & 9 & 66 \\
16 & 10 & 52 \\
7 & 6 & 29 \\
9 & 11 & 16 \\
2 & 1 & 78 \\
7 & 2 & 69 \\
11 & 0 & 31 \\
9 & 1 & 26 \\
6 & 0 & 75 \\
15 & 4 & 59 \\
20 & 2 & 20 \\
22 & 1 & 13
\end{tabular}

$\begin{array}{lll}1 & 5 & 75\end{array}$

$\begin{array}{lll}10 & 7 & 61\end{array}$

$\begin{array}{lll}13 & 8 & 21\end{array}$

$13 \quad 5 \quad 18$

$\begin{array}{lll}5 & 17 & 59\end{array}$

$8 \quad 15 \quad 55$

$\begin{array}{lll}7 & 19 & 16\end{array}$

$\begin{array}{lll}8 & 15 & 13\end{array}$

$\begin{array}{lll}48 & 11 & 22\end{array}$

$\begin{array}{lll}57 & 8 & 13\end{array}$

$35 \quad 6 \quad 1$

$34 \quad 2 \quad 0$

$\begin{array}{lll}47 & 7 & 27\end{array}$

$\begin{array}{lll}48 & 9 & 21\end{array}$

$27 \quad 3 \quad 12$

$29 \quad 5 \quad 2$

$21 \quad 30 \quad 30$

$\begin{array}{lll}22 & 32 & 24\end{array}$

$\begin{array}{lll}12 & 15 & 15\end{array}$

$\begin{array}{lll}14 & 10 & 12\end{array}$

$\begin{array}{lll}11 & 29 & 41\end{array}$

$\begin{array}{lll}21 & 27 & 30\end{array}$

$\begin{array}{lll}12 & 19 & 11\end{array}$

$13 \quad 11 \quad 12$

$\begin{array}{lll}19 & 9 & 53\end{array}$

$22 \quad 10 \quad 46$

$19 \quad 10 \quad 13$

$\begin{array}{lll}15 & 7 & 14\end{array}$ 
Table 11. Number of responses to each symbol as a function of age and gender (continued).

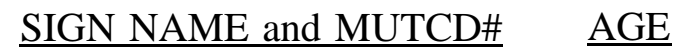
Response

34. Two-Way

Traffic

35. Hill

36. Pavement

Ends *

W6-3

$\mathrm{M}$

0

$\mathrm{E}$

W7-1

37. Slippery When Wet

38. Bicycle Crossing

39. Pedestrian Crossing

40. Deer

Crossing

W11-3

W11-1

W8-5

W8-3a

Y

M

0

E

Y

M

0

E

Y

$\mathrm{M}$

0

E

Y

M

0

E

W11a-2

Y

M

0

E

Y

$\mathrm{M}$

0

E

41. Cattle

Crossing

W11-4

Crossing

42. Tractor

Crossing

W11-5

Crossing

43. Double

Arrow
MALES

$0 \quad 1$

FEMALES

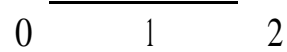

$\begin{array}{rrr}7 & 3 & 71 \\ 8 & 1 & 69 \\ 8 & 1 & 33 \\ 6 & 0 & 30 \\ 7 & 11 & 63 \\ 8 & 5 & 65 \\ 5 & 2 & 35 \\ 11 & 3 & 22\end{array}$

$\begin{array}{lll}17 & 1 & 63\end{array}$

$\begin{array}{lll}37 & 3 & 38\end{array}$

$\begin{array}{lll}24 & 1 & 17\end{array}$

$\begin{array}{lll}27 & 1 & 8\end{array}$

$\begin{array}{lll}48 & 1 & 32\end{array}$

$33 \quad 2 \quad 43$

$\begin{array}{lll}23 & 0 & 19\end{array}$

$\begin{array}{lll}19 & 0 & 17\end{array}$

$41 \quad 14 \quad 26$

$39 \quad 10 \quad 29$

$28 \quad 4 \quad 10$

$\begin{array}{lll}22 & 6 & 8\end{array}$

$\begin{array}{lll}2 & 11 & 68\end{array}$

$\begin{array}{lll}4 & 7 & 67\end{array}$

$\begin{array}{lll}3 & 7 & 32\end{array}$

$\begin{array}{lll}5 & 6 & 25\end{array}$

$\begin{array}{lll}0 & 2 & 79\end{array}$

$\begin{array}{lll}0 & 1 & 77\end{array}$

$\begin{array}{lll}0 & 0 & 42\end{array}$

$0 \quad 0 \quad 36$

$\begin{array}{lll}0 & 1 & 80\end{array}$

$\begin{array}{lll}1 & 1 & 74\end{array}$

$\begin{array}{lll}0 & 0 & 42\end{array}$

$\begin{array}{lll}0 & 0 & 36\end{array}$

$\begin{array}{lll}1 & 1 & 79\end{array}$

$\begin{array}{lll}4 & 4 & 70\end{array}$

$\begin{array}{lll}1 & 1 & 40\end{array}$

$5 \quad 5 \quad 26$

$41 \quad 16 \quad 24$

$\begin{array}{lll}52 & 12 & 14\end{array}$

$26 \quad 10 \quad 6$

$\begin{array}{lll}25 & 8 & 3\end{array}$ 
Table 11. Number of responses to each symbol as a function of age and gender (continued).

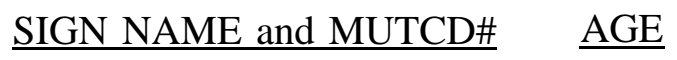
Response

44. Low

Vertical

Clearance

45. Playground

46. Advance flagger *

47. Worker (construction)

48. Rest Area

49. Phone

D9-1

D5-5a

50. Hospital *

D9-2

51. Camping *

$$
\text { D9-3 }
$$

52. Recreational Vehicle

53. Handicapped *

\section{MALES}

$0 \longdiv { 1 } 2$

$\mathrm{Y}$
$\mathrm{M}$
0
$\mathrm{E}$

E

M

0

E

Y

M

0

E

Y

$\mathrm{M}$

0

E

Y

$$
\text { Y }
$$

0

E

$\mathrm{Y}$

$\mathrm{M}$

0

$\mathrm{E}$

$\begin{array}{llll} & 0 & 0 & 43\end{array}$

$\begin{array}{llll}\mathrm{Y} & 4 & 0 & 76\end{array}$

$\begin{array}{llll}\text { M } & 4 & 0 & 77\end{array}$

$\begin{array}{llll}0 & 4 & 0 & 34\end{array}$

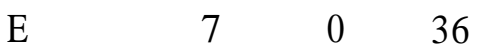

$\begin{array}{llll}\mathrm{Y} & 23 & 13 & 44\end{array}$

M 20

$0 \quad 1$

E

E 23

$\mathrm{M}$

0

E

$\mathrm{Y}$

$\mathrm{M}$

0

E
FEMALES

$\begin{array}{rrr}0 & 1 & 2 \\ 3 & 2 & 76 \\ 3 & 3 & 72 \\ 3 & 1 & 38 \\ 3 & 0 & 33 \\ 3 & 10 & 68 \\ 2 & 3 & 73 \\ 2 & 2 & 38 \\ 2 & 6 & 28 \\ 22 & 3 & 56 \\ 23 & 2 & 53 \\ 20 & 3 & 19 \\ 21 & 1 & 14 \\ 9 & 4 & 68 \\ 12 & 5 & 61 \\ 10 & 1 & 31 \\ 8 & 3 & 25 \\ 3 & 0 & 78 \\ 5 & 1 & 72 \\ 4 & 1 & 37 \\ 5 & 1 & 30 \\ 0 & 1 & 80 \\ 0 & 0 & 78 \\ 0 & 0 & 42 \\ 0 & 0 & 36\end{array}$

$3 \quad 0 \quad 78$

$\begin{array}{lll}9 & 2 & 68\end{array}$

$\begin{array}{lll}9 & 0 & 33\end{array}$

$\begin{array}{lll}5 & 0 & 31\end{array}$

$\begin{array}{lll}18 & 18 & 45\end{array}$

$26 \quad 22 \quad 30$

$12 \quad 9 \quad 21$

$\begin{array}{lll}17 & 4 & 15\end{array}$

$\begin{array}{rrr}7 & 29 & 45 \\ 11 & 33 & 34 \\ 6 & 24 & 12 \\ 9 & 10 & 17 \\ 1 & 35 & 45 \\ 8 & 28 & 42 \\ 3 & 16 & 23 \\ 5 & 22 & 9\end{array}$


Table 11. Number of responses to each symbol as a function of age and gender (continued).

SIGN NAME and MUTCD\# AGE Response

54. Gas

55. Food

56. Lodging

57. Tourist Information *

58. Diesel

59. RV

Sanitary

Station

60. Emergency

Medical

Services *

61. Propane

62. Hiking

Trail *

63. Airport*
D9-7

D9-8

D9-9

D9-10

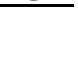

$\mathrm{M}$

0

E

$\mathrm{Y}$

M

0

E

$Y$

$$
\mathrm{M}
$$

0

E

Y

$\mathrm{Y}$

$\mathrm{M}$

0

E

D9-11

D9-12

$\mathrm{Y}$
$\mathrm{M}$

0

E

$\mathrm{Y}$

0

E

D9-13

D)-13

Y

$\mathrm{M}$

0

E

D9-15

Y

(

0

E

I-4

$\mathrm{Y}$
$\mathrm{M}$

0

E

I-5
MALES

$\begin{array}{rrr}0 & 1 & 2 \\ 0 & 3 & 77 \\ 1 & 0 & 80 \\ 0 & 2 & 36 \\ 0 & 0 & 43\end{array}$

$\begin{array}{lll}4 & 0 & 76\end{array}$

$\begin{array}{lll}2 & 0 & 79\end{array}$

$1 \quad 1 \quad 36$

$3 \quad 0 \quad 40$

$13 \quad 2 \quad 65$

$\begin{array}{lll}16 & 0 & 65\end{array}$

$8 \quad 0 \quad 30$

$\begin{array}{lll}12 & 2 & 29\end{array}$

$\begin{array}{lll}35 & 4 & 41\end{array}$

$40 \quad 0 \quad 41$

$\begin{array}{lll}18 & 2 & 18\end{array}$

31

31

$1 \quad 10 \quad 69$

$\begin{array}{lll}3 & 8 & 70\end{array}$

$\begin{array}{lll}0 & 8 & 30\end{array}$

$\begin{array}{lll}1 & 10 & 32\end{array}$

$\begin{array}{lll}28 & 3 & 49\end{array}$

$19 \quad 0 \quad 62$

$\begin{array}{lll}11 & 1 & 26\end{array}$

$\begin{array}{lll}13 & 3 & 27\end{array}$

$14 \quad 15 \quad 51$

$\begin{array}{lll}14 & 12 & 55\end{array}$

$\begin{array}{lll}11 & 5 & 22\end{array}$

$\begin{array}{lll}16 & 7 & 20\end{array}$

$\begin{array}{lll}22 & 1 & 57\end{array}$

$\begin{array}{lll}21 & 0 & 60\end{array}$

$\begin{array}{lll}10 & 1 & 27\end{array}$

$\begin{array}{lll}7 & 0 & 36\end{array}$

$\begin{array}{lll}11 & 9 & 60\end{array}$

$\begin{array}{lll}8 & 8 & 65\end{array}$

$\begin{array}{lll}9 & 8 & 21\end{array}$

$\begin{array}{lll}12 & 5 & 26\end{array}$

Y

E

0

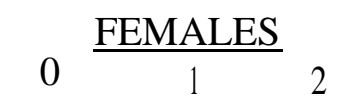

12

$\begin{array}{lll}0 & 1 & 80\end{array}$

$\begin{array}{lll}0 & 5 & 73\end{array}$

$\begin{array}{lll}0 & 2 & 40\end{array}$

$\begin{array}{lll}1 & 1\end{array}$

$\begin{array}{lll}2 & 3 & 76\end{array}$

$\begin{array}{lll}3 & 2 & 73\end{array}$

$1 \quad 0 \quad 41$

$2 \quad 1 \quad 33$

$\begin{array}{lll}9 & 3 & 69\end{array}$

$\begin{array}{lll}14 & 3 & 61\end{array}$

$\begin{array}{lll}9 & 1 & 32\end{array}$

$\begin{array}{lll}12 & 3 & 20\end{array}$

$\begin{array}{lll}26 & 2 & 53\end{array}$

$35 \quad 4 \quad 39$

$24 \quad 0 \quad 18$

$\begin{array}{lll}24 & 1\end{array}$

$\begin{array}{lll}0 & 18 & 63\end{array}$

$\begin{array}{lll}0 & 19 & 59\end{array}$

$\begin{array}{lll}4 & 13 & 25\end{array}$

$\begin{array}{lll}2 & 17 & 17\end{array}$

$\begin{array}{lll}28 & 5 & 48\end{array}$

$\begin{array}{lll}24 & 4 & 50\end{array}$

$14 \quad 0 \quad 28$

$\begin{array}{lll}17 & 0 & 19\end{array}$

$\begin{array}{lll}18 & 14 & 49\end{array}$

$18 \quad 16 \quad 44$

$\begin{array}{lll}11 & 9 & 22\end{array}$

$\begin{array}{lll}19 & 3 & 14\end{array}$

$\begin{array}{lll}32 & 1 & 48\end{array}$

$\begin{array}{lll}28 & 2 & 48\end{array}$

$\begin{array}{lll}13 & 0 & 29\end{array}$

$\begin{array}{lll}20 & 1\end{array}$

$\begin{array}{lll}11 & 11 & 59\end{array}$

$\begin{array}{lll}12 & 7 & 59\end{array}$

$\begin{array}{lll}6 & 2 & 34\end{array}$

$\begin{array}{lll}11 & 8 & 17\end{array}$

$\begin{array}{lll}4 & 3 & 74\end{array}$

$3 \quad 6 \quad 69$

$4 \quad 2 \quad 36$

$\begin{array}{lll}15 & 2 & 19\end{array}$ 
Table 11. Number of responses to each symbol as a function of age and gender (continued).

SIGN NAME and MUTCD\# $\underline{\text { AGE }}$ Response

64. Bus

Station

I-6

65. Train

Station *

I-7

66. Library *

67. Dock

I-9

68. Winter

Recreation

I-100

I-8

69. Lighthouse

RG-120

70. Ranger

Station *

RG-170

71. Rest Rooms * RM-140

72. Campfire *

RA-030

73. Shelter

(Sleeping)

M

0

E
MALES

$0 \quad \frac{M}{1} 2$

FEMALES

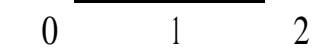

$\begin{array}{lll}69 & 5 & 7\end{array}$

$\begin{array}{lll}71 & 0 & 7\end{array}$

$\begin{array}{lll}41 & 1 & 0\end{array}$

$34 \quad 0 \quad 2$

E $\quad 40 \quad 2$

$\begin{array}{llll}\mathrm{Y} & 4 & 4 & 72\end{array}$

$\begin{array}{lll}1 & 6 & 74\end{array}$

$1 \quad 7 \quad 73$

$\begin{array}{lll}4 & 2 & 72\end{array}$

$\begin{array}{lll}7 & 0 & 35\end{array}$

$\begin{array}{lll}6 & 6 & 24\end{array}$

$\begin{array}{lll}28 & 5 & 48\end{array}$

$30 \quad 6 \quad 42$

$\begin{array}{lll}20 & 1 & 21\end{array}$

$\begin{array}{lll}24 & 2 & 10\end{array}$

$\begin{array}{llll}\text { E } & 25 & 0 & 18\end{array}$

$\begin{array}{lll}33 & 21 & 27\end{array}$

$\begin{array}{lll}45 & 8 & 25\end{array}$

$\begin{array}{lll}17 & 7 & 18\end{array}$

$\begin{array}{lll}26 & 3 & 7\end{array}$

$\begin{array}{llll}\mathrm{E} & 19 & 6 & 18\end{array}$

$\begin{array}{lll}65 & 4 & 12\end{array}$

$\begin{array}{lll}71 & 3 & 4\end{array}$

$\begin{array}{lll}38 & 1 & 3\end{array}$

$36 \quad 0 \quad 0$

$\begin{array}{lll}8 & 4 & 69\end{array}$

$\begin{array}{lll}9 & 3 & 66\end{array}$

$\begin{array}{lll}5 & 1 & 36\end{array}$

$\begin{array}{lll}10 & 3 & 23\end{array}$

$\begin{array}{lll}26 & 5 & 50\end{array}$

$31 \quad 1 \quad 46$

$\begin{array}{lll}16 & 4 & 22\end{array}$

$\begin{array}{lll}22 & 2 & 12\end{array}$

$\begin{array}{lll}3 & 0 & 78\end{array}$

$\begin{array}{lll}4 & 1 & 73\end{array}$

$\begin{array}{lll}6 & 1 & 35\end{array}$

$\begin{array}{lll}6 & 1 & 28\end{array}$

$\begin{array}{lll}11 & 6 & 64\end{array}$

$\begin{array}{lll}18 & 3 & 57\end{array}$

$\begin{array}{lll}13 & 4 & 25\end{array}$

$\begin{array}{lll}15 & 3 & 18\end{array}$

$\begin{array}{lll}58 & 17 & 6\end{array}$

$\begin{array}{lll}57 & 17 & 4\end{array}$

$\begin{array}{lll}34 & 5 & 3 \\ 25 & 9 & 2\end{array}$


Table 11. Number of responses to each symbol as a function of age and gender (continued).

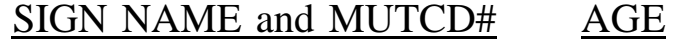
Response

74. Showers *

75. Amphitheater

76. Canoeing

RW-020

77. Launch Ramp

RW-080

78. Swimming * RW-130

79. Ice Skating *

RS-010

80. Sledding * RS-060

81. Snowmobiling *

82. School Advance

83. School Crossing
MALES

$\begin{array}{lll}0 & 1 & 2\end{array}$

FEMALES

$\begin{array}{rrr}0 & 1 & 2 \\ 2 & 1 & 78 \\ 16 & 1 & 61 \\ 10 & 1 & 31 \\ 22 & 1 & 13\end{array}$

$54 \quad 11 \quad 16$

$\begin{array}{lll}60 & 3 & 15\end{array}$

$30 \quad 4 \quad 8$

$32 \quad 2 \quad 2$

$12 \quad 16 \quad 55$

$\begin{array}{lll}8 & 21 & 49\end{array}$

$\begin{array}{lll}1 & 16 & 25\end{array}$

$\begin{array}{lll}7 & 16 & 13\end{array}$

$\begin{array}{lll}9 & 4 & 68\end{array}$

$15 \quad 3 \quad 60$

$\begin{array}{lll}7 & 0 & 35\end{array}$

$\begin{array}{lll}5 & 4 & 27\end{array}$

$\begin{array}{lll}4 & 2 & 75\end{array}$

$\begin{array}{lll}7 & 5 & 66\end{array}$

$\begin{array}{lll}17 & 1 & 24\end{array}$

$21 \quad 2 \quad 13$

$34 \quad 11 \quad 36$

$\begin{array}{lll}41 & 5 & 32\end{array}$

$\begin{array}{lll}22 & 6 & 14\end{array}$

$\begin{array}{lll}31 & 1 & 4\end{array}$

$\begin{array}{lll}16 & 8 & 57\end{array}$

$16 \quad 4 \quad 58$

$13 \quad 3 \quad 26$

$18 \quad 2 \quad 16$

$16 \quad 5 \quad 60$

$23 \quad 3 \quad 52$

$12 \quad 4 \quad 26$

$\begin{array}{lll}12 & 3 & 21\end{array}$

$\begin{array}{lll}47 & 29 & 5\end{array}$

$\begin{array}{lll}49 & 24 & 5\end{array}$

$26 \quad 15 \quad 1$

$26 \quad 10 \quad 0$

$\begin{array}{lll}22 & 13 & 46\end{array}$

$\begin{array}{lll}18 & 18 & 42\end{array}$

$\begin{array}{lll}15 & 7 & 22\end{array}$

$\begin{array}{lll}8 & 4 & 24\end{array}$ 
Table 11. Number of responses to each symbol as a function of age and gender (continued).

\begin{tabular}{|c|c|c|c|c|c|c|c|c|}
\hline \multicolumn{2}{|c|}{ SIGN NAME and MUTCD\# } & \multirow[t]{2}{*}{$\underline{\mathrm{AGE}}$} & \multicolumn{3}{|c|}{$\underline{\text { MALES }}$} & \multicolumn{3}{|c|}{$\underline{\text { FEMALES }}$} \\
\hline \multicolumn{2}{|c|}{ Response } & & 0 & 1 & 2 & 0 & 1 & 2 \\
\hline \multirow{4}{*}{$\begin{array}{l}\text { 84. R.R. } \\
\text { Advance } \\
\text { Warning }\end{array}$} & W10-1 & Y & 0 & 61 & 19 & 1 & 66 & 14 \\
\hline & & M & 2 & 70 & 9 & 3 & 65 & 10 \\
\hline & & 0 & 1 & 30 & 7 & 3 & 34 & 5 \\
\hline & & $\mathrm{E}$ & 3 & 33 & 7 & 2 & 31 & 3 \\
\hline \multirow{4}{*}{$\begin{array}{l}\text { 85. R.R. * } \\
\text { Advance } \\
\text { Warning } \\
\text { (Parallel) }\end{array}$} & W10-3 & $\mathrm{Y}$ & 8 & 23 & 49 & 8 & 28 & 45 \\
\hline & & M & 6 & 22 & 53 & 10 & 35 & 33 \\
\hline & & 0 & 9 & 14 & 15 & 6 & 20 & 16 \\
\hline & & $\mathrm{E}$ & 7 & 14 & 22 & 13 & 16 & 7 \\
\hline \multirow[t]{4}{*}{ 86. Rest Area } & & Y & 2 & 1 & 17 & 1 & 3 & 18 \\
\hline & & $\mathrm{M}$ & 4 & 3 & 24 & 1 & 1 & 25 \\
\hline & & 0 & 2 & 0 & 8 & 2 & 0 & 8 \\
\hline & & $\mathrm{E}$ & 2 & 3 & 8 & 0 & 0 & 5 \\
\hline
\end{tabular}

Table 12. Percentage comprehension and mean familiarity ratings for each symbol.

\section{$\underline{\text { Sign }} \quad \underline{\text { Percent } \text { correct* }^{*} \quad \underline{\text { Mean Familiarity }}}$}

$\begin{array}{llrl}\text { 1. } & \text { No Right Turn } & 96.9 & 1.44 \\ 2 . & \text { NoUTurn } & 97.9 & 1.23 \\ \text { 3. } & \text { Straight Or Left } & 16.0 & 2.18 \\ \text { 4. } & \text { Keep Right } & 85.8 & 2.10 \\ \text { 5. } & \text { No Trucks } & 97.1 & 1.91 \\ \text { 6. } & \text { No Bicycles } & 97.8 & 1.86 \\ \text { 7. } & \text { No Parking } & 95.4 & 1.38 \\ \text { 8. } & \text { No Hitchhiking } & 95.8 & 3.32 \\ \text { 9. } & \text { National Network } & 61.0 & 4.45 \\ & \text { Route } & & \\ \text { 10. } & \text { National Network } & 77.7 & 3.80 \\ & \text { Prohibited } & & \\ \text { 11. } & \text { Mandatory Seat Belt } & 74.4 & 3.45 \\ \text { 12. } & \text { Right Turn } & 91.7 & 1.76 \\ \text { 13. } & \text { Right Curve } & 94.9 & 1.62 \\ \text { 14. } & \text { Right Reverse Turn } & 70.4 & 2.24 \\ \text { 15. } & \text { Right Reverse Curve } & 69.2 & 1.88 \\ \text { 16. } & \text { Winding Road (Right) } & 94.0 & 1.22 \\ \text { 17. } & \text { Large Arrow } & 38.9 & 2.19\end{array}$

* Percentage of responses scored correct and partially correct.

** Sign 48 was the symbol with large picnic table only; sign 86 was the sign with small picnic table and tree (D5-5a). 
Table 12. Percentage comprehension and mean familiarity ratings for each symbol (continued).

$\underline{\text { Sign }} \quad \underline{\text { Percent Correct* }} \quad \underline{\text { Mean Familiarity }}$

\begin{tabular}{|c|c|c|}
\hline Double Head Arrow & 68.1 & 2.74 \\
\hline Chevron Alignment & 36.8 & 3.56 \\
\hline Cross Road & 92.9 & 1.98 \\
\hline $\begin{array}{l}\text { Side Road } \\
\text { (Right } 90 \text { Deg) }\end{array}$ & 87.4 & 2.16 \\
\hline $\begin{array}{l}\text { Side Road } \\
\text { (Right } 45 \text { Deg) }\end{array}$ & 72.5 & 2.64 \\
\hline T Symbol & 78.1 & 2.21 \\
\hline Y Symbol & 86.6 & 3.07 \\
\hline Stop Ahead & 90.2 & 1.93 \\
\hline Yield Ahead & 75.8 & 3.00 \\
\hline Signal Ahead & 87.3 & 1.41 \\
\hline Merge & 90.0 & 1.97 \\
\hline Added Lane & 25.5 & 2.82 \\
\hline Lane Reduction * & 38.1 & 2.14 \\
\hline Narrow Bridge & 77.3 & 3.68 \\
\hline Divided Highway & 78.3 & 2.01 \\
\hline Divided Highway Ends & 71.7 & 2.08 \\
\hline Two-Way Traffic & 89.2 & 1.49 \\
\hline Hill & 90.4 & 2.04 \\
\hline Pavement Ends & 64.4 & 4.15 \\
\hline Slippery When Wet & 44.6 & 1.60 \\
\hline Bicycle Crossing & 44.8 & 2.24 \\
\hline Pedestrian Crossing & 91.9 & 1.69 \\
\hline Deer Crossing & 98.7 & 1.38 \\
\hline Cattle Crossing & 99.8 & 2.32 \\
\hline Tractor Crossing & 95.2 & 3.28 \\
\hline Double Arrow & 34.8 & 4.33 \\
\hline Low Vehicle Clearance & 96.6 & 1.70 \\
\hline Playground & 96.6 & 3.07 \\
\hline Advance Flagger & 69.5 & 1.71 \\
\hline Worker (Construction) & 83.1 & 2.75 \\
\hline Rest Area** & 93.1 & 2.10 \\
\hline Phone & 99.8 & 1.35 \\
\hline Hospital & 90.6 & 1.65 \\
\hline Camping & 68.7 & 3.47 \\
\hline Recreation Vehicle & 89.2 & 2.96 \\
\hline Handicapped & 91.2 & 1.44 \\
\hline Gas & 99.6 & 1.68 \\
\hline Food & 96.4 & 1.96 \\
\hline
\end{tabular}


Table 12. Percentage comprehension and mean familiarity ratings for each symbol (continued).

$\underline{\text { Sign }}$

56. Lodging

57. Tourist Information

58. Diesel Fuel

59. RV Sanitary Station

60. Emergency Medical Services

61. Propane

62. Hiking Trail

63. Airport

64. Bus Station

65. Train Station

66. Library

67. Dock

68. Winter Recreation

69. Lighthouse

70. Ranger Station

71. Rest Rooms

72. Campfire

73. Shelter (Sleeping)

74. Showers

75. Amphitheater

76. Canoeing

77. Launch Ramp

78. Swimming

79. Ice Skating

80. Sledding

81. Snowmobiling

82. School Advance

83. School Crossing

84. RR Advance Warning

85. RR Advance Warning (Parallel)

86. Rest Area**
Percent Correct *

80.6

52.6

97.7

68.7

74.8

68.1

83.5

89.4

10.4

94.2

55.0

53.5

11.0

89.6

62.1

91.2

73.3

26.8

80.8

28.3

87.5

87.3

79.0

52.9

75.2

71.5

41.3

77.1

97.0

86.0

89.9 $\underline{\text { Mean Familiarity }}$

2.82

3.50

2.95

2.93

3.72

3.98

3.08

2.01

3.51

3.78

4.49

4.31

4.47

4.27

3.09

2.24

2.98

4.23

3.97

4.60

3.44

2.27

2.80

3.91

4.04

3.27

1.59

1.80

1.56

3.69

4.14 
Table 13. Percentage of correct responses

as a function of sign category.*

\begin{tabular}{|c|c|c|}
\hline Sign category & $\underline{\text { Sign numbers }}$ & $\underline{\text { Percent correct }}$ \\
\hline Regulatory & $1-11$ & 81.4 \\
\hline Warning & $12-45$ & 75.4 \\
\hline Construction & $46-47$ & 76.3 \\
\hline Information $* *$ & $48-68$ & 74.7 \\
\hline Recreation and Cultural & $69-81$ & 69.7 \\
\hline School & $82-83$ & 59.2 \\
\hline Railroad Crossing & $84-85$ & 91.5 \\
\hline
\end{tabular}

\section{COMBINING DATA FROM COMPREHENSION AND LEGIBILITY DISTANCE}

The subsequent parts of phase 1 require further testing of a set of 18 symbols, including good, intermediate, and poor ones. It was decided in advance to include in this test set two examples from each of the three main types of message: regulatory, warning (including work zone, railroad, and school signs), and information (guide, motorist services, and recreation). In order to determine an overall index of the effectiveness of each symbol based on the first two studies in phase 1 , the data from each measure (comprehension and legibility distance) were rank ordered from best to worst performance. As it was unlikely that the ranks on the two measures would correspond closely, the decision was made to rank order the signs first on the basis of legibility distance. The signs selected as the "best" in that category were the two with the greatest legibility distance, which were also very high in the comprehension measure.

Based first on their ranking on mean daytime legibility, and secondly, on their mean comprehension score, six Best, six Intermediate, and six Worst signs were selected for further evaluation. A sign was not selected for a category if its overall comprehension score was also not in the appropriate quartile for the category. Thus, signs in the Best category were those with the greatest legibility, whose comprehension rank was as high as possible, but not below the first quartile; signs in the Intermediate category were those closest to the middle ranks on legibility and whose comprehension was as close to the middle of the distribution as possible and not outside the middle 25 percent; and signs in the Worst category were those with both the lowest possible legibility rank and a comprehension rank as close as possible to the bottom of the fourth quartile. This selection process was carried out separately for Regulatory, Warning, and Recreational and Guide signs, so that two Regulatory, two Warning, one Recreational, and one Guide sign could each could be included in the Best, Intermediate, and Worst categories (see 'appendix F).

The same procedure was used to select the "poorest" signs in that category, that is, the two signs with the shortest legibility distance, which were also very poorly understood. For the 
signs at the intermediate level, those two closest to the median rank on the visibility measure, which were also close to the median on the comprehension measure, were selected. Some judgment was exercised in this process to avoid selecting two signs that were similar in design (e.g., NO RIGHT TURN and NO U TURN). The six signs for the remaining categories were selected in the same manner. This approach led to selection of the signs shown in table 14.

Table 14. Test set of 18 signs ( 6 best, 6 intermediate, 6 worst) based on legibility distance and comprehension rankings.

\begin{tabular}{lllllll} 
Sign & \multicolumn{2}{c}{ 6 Best } & \multicolumn{2}{c}{ 6 Intermediate } & \multicolumn{2}{c}{ 6 Worst } \\
Ty p e & Name & $\underline{\text { MUTCD\# }}$ & Name & $\underline{\text { MUTCD\# }}$ & Name & $\underline{\text { MUTCD\# }}$ \\
Warn & Cross Road & W2-1 & Div Hwy Ends W6-2 & Bicycle Crossing & W11-1 \\
Warn & Right Curve & W1-2R & Adv Flagger & W20-7a & Pavement Ends & W8-3a \\
Reg & No U-Turn & R3-4 & No Parking & R8-3a & Nat Net Prohib & R14-5 \\
Reg & Keep Right & R4-7 & No Trucks & R5-2 & Mand Seatbelt & R16-1 \\
G/R & Hospital. & D9-2 & RV Sanitary & D9-12 & Skating & RS-010 \\
G/R & Phone & D9-1 & Campfire & RA-030 & Ranger Station & RG-170
\end{tabular}

Notes:

Warn = Warning Signs

Reg = Regulatory Signs

G/R = Guide and Recreational/Cultural Signs 


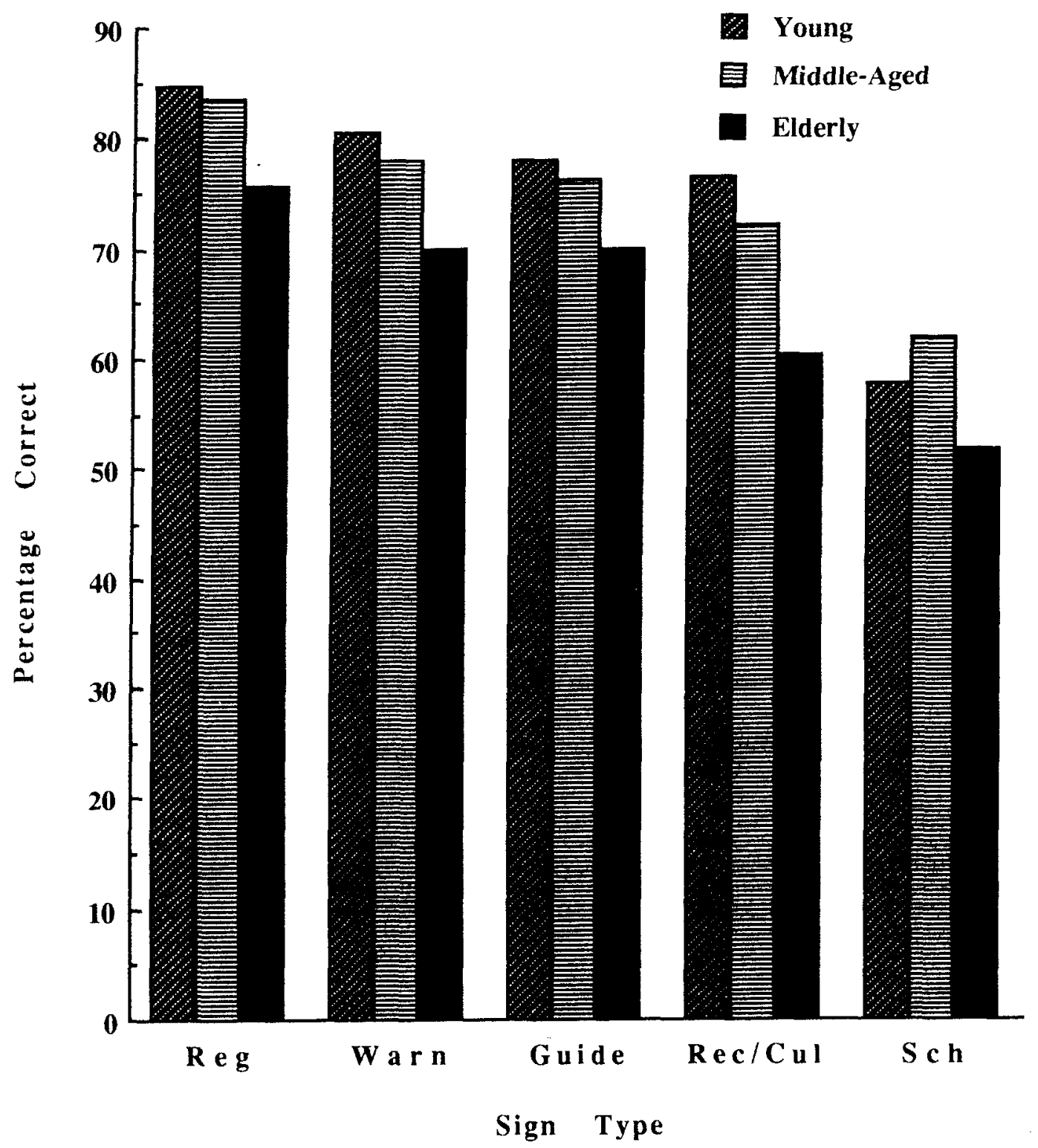

Figure 3. Mean percent comprehension of different sign types as a function of driver age (phase 1). 


\section{CHAPTER 5: NIGHTTIME LEGIBILITY OF STANDARD SYMBOL HIGHWAY SIGNS}

Psychophysical and physiological evidence suggests that age-related deficits in the recognition of highway signs may be exacerbated under low illumination conditions. Hence, the development and evaluation of design guidelines for the optimization of symbol highway signs must consider the potential age differences in performance under nighttime as well as daytime conditions.

\section{METHOD}

\section{Subjects}

Three age groups of active, community-resident drivers were sampled: a young group ( $\mathrm{N}$ $=12, \underline{\mathrm{M}}$ age $=18.8$, range 18 to 20$)$, a middle-aged group $(\mathrm{N}=12, \underline{\mathrm{M}}$ age $=50.8$, range 40 to 59$)$, and an old group $(\mathrm{N}=20, \underline{\mathrm{M}}$ age $=72.0$, range 65 to 87$)$. The young subjects were recruited from the student population of Oakland University (Rochester, Michigan), and the middle-aged and older subjects were recruited from community service organizations in Auburn Hills and Pontiac, Michigan, respectively. Demographic characteristics of the three age groups are presented in detail in table 15 . Visual health of the three age groups, as indexed by photopic acuity, was representative of known population values (see table 16). However, one middle-aged subject and three older subjects were excluded from the data analysis because of excessive visual pathology (viz., retinal degeneration and/or profound contrast sensitivity loss due to glaucoma).

\section{Stimuli And Apparatus}

Binocular photopic $\left(85 \mathrm{~cd} / \mathrm{m}^{2}\right)$ acuity was measured using a customized Landolt $\mathrm{C}$ chart. This chart divided the acuity range between 6/12 and 6/3 (20/40 and 20/10) (Snellen) into 15 equivalently spaced optotype intervals, yielding a highly precise estimate of spatial resolving power. Contrast sensitivity was assessed using a CRT-based analog of the Vistech VCTS 6500 far chart (used at the University of Calgary laboratory). The advantage of the CRT-based technique was that more precise estimates of contrast sensitivity could be obtained. Contrast sensitivities were measured at each of 5 spatial frequencies: 1, 2, 4, 8, and 18 cycles per degree of visual angle (c/deg), and at each of two luminance levels: low photopic $\left(22 \mathrm{~cd} / \mathrm{m}^{2}\right)$ and mesopic $\left(6 \mathrm{~cd} . / \mathrm{m}^{2}\right)$.

The test stimuli consisted of 18 symbol signs selected on the basis of their legibility and comprehension levels in the preceding studies (see table 18). They were presented in their regulation colors using an Apple IIsi computer equipped with a 33-cm (13-in) RGB monitor. White areas of the screen were maintained at $8.5 \mathrm{~cd} / \mathrm{m}^{2}$. A viewing distance of $5 \mathrm{~m}(16.4 \mathrm{ft})$ was maintained by the use of a head/chin rest. 
Table 15. Participant demographics: nighttime legibility (phase 1) (Percentage of Drivers).

\begin{tabular}{|c|c|c|c|}
\hline & & Age Group & \\
\hline$\frac{\text { Distance driven }}{\underline{\text { miles }]}}$ & Young & $\underline{\text { Middle-aged }}$ & $\underline{\text { Older }}$ \\
\hline under 2,000 & 17 & & 35 \\
\hline $2,000-4,999$ & 33 & & 53 \\
\hline $5,000-9,999$ & 25 & 18 & 6 \\
\hline $10,000-14,999$ & 8 & 27 & 5 \\
\hline $15,000-19,999$ & 17 & 18 & - \\
\hline 20,000 or more & - & 37 & - \\
\hline Driving Environment & & & \\
\hline rural/sparsely populated & 8 & & \\
\hline small town & 25 & 9 & 6 \\
\hline suburbs of city & 67 & 82 & 59 \\
\hline inner city & - & 9 & 35 \\
\hline Type of Vehicle & & & \\
\hline $\begin{array}{l}\text { passenger car } \\
\text { motor home/RV } \\
\text { motorcycle }\end{array}$ & 100 & 91 & 88 \\
\hline light truck/van & - & 9 & 12 \\
\hline heavy truck & & - & \\
\hline bus & - & - & - \\
\hline $\mathrm{cab}$ & - & - & \\
\hline$\frac{\text { Percentage Driven }}{\text { at Night }}$ & & & \\
\hline $0-5$ & & 9 & 76 \\
\hline $6-10$ & 8 & 9 & 6 \\
\hline $11-15$ & - & 18 & \\
\hline $16-20$ & 16 & 37 & 12 \\
\hline $21-30$ & 33 & & 6 \\
\hline $31-40$ & 16 & 9 & - \\
\hline $41-50$ & 27 & 18 & - \\
\hline 51-or more & - & - & - \\
\hline$\frac{\text { Highest Education }}{\underline{\text { Level }}}$ & & & \\
\hline $1-8$ years & - & - & 16 \\
\hline some high school & & & 36 \\
\hline high school graduate & 16 & & 30 \\
\hline $\begin{array}{l}\text { trade/business } \\
\text { college }\end{array}$ & & & 12 \\
\hline
\end{tabular}


Table 15. Participant demographics: nighttime legibility (phase 1) (Percentage of Drivers) (Continued).

\begin{tabular}{|c|c|c|c|}
\hline \multirow[b]{2}{*}{ Highest Education } & \multicolumn{3}{|c|}{$\underline{\text { Age Group }}$} \\
\hline & Young & $\underline{\text { Middle-aged }}$ & $\underline{\text { Older }}$ \\
\hline $\begin{array}{l}\text { Level } \\
\text { some college or } \\
\text { university }\end{array}$ & 84 & 55 & 6 \\
\hline university degree & & 18 & \\
\hline $\begin{array}{l}\text { post graduate } \\
\text { none }\end{array}$ & & 27 & \\
\hline
\end{tabular}

Table 16. Vision assessment parameters as a function of age: nighttime legibility (phase 1).

$\begin{array}{lccc}\text { Mesopic Luminance } & \text { Young } & \underline{\underline{\text { Middle-aged }}} & \text { Older } \\ \text { Low Frequency Cutoff (c/deg) } & 2.25 & 1.54 & 1.39 \\ \text { Log Peak CS } & 2.30 & 2.15 & 1.88 \\ \text { High Frequency Cutoff (c/deg) } & 8.71 & 7.61 & 6.54\end{array}$

$\underline{\text { Photopic Luminance }}$

Low Frequency Cutoff (c/deg)

1.29

1.37

$\log$ Peak CS

2.13

2.19

High Frequency Cutoff (c/deg)

8.23

8.44

Acuity (minarc)

$\begin{array}{ccc}0.62 & 0.81 & 1.50 \\ (\mathrm{SD}=0.07) & (\mathrm{SD}=0.36) & (\mathrm{SD}=0.58)\end{array}$

Procedure

After completion of the informed consent procedure, subjects filled out the standard driving history/status questionnaire (see chapter 3). At this point, their photopic acuity and contrast sensitivity functions were measured. The laboratory was then darkened and the recognition size/distance thresholds for the practice and test symbol signs were measured using the same procedure detailed in the Method section of the Daytime Legibility study. At the end of the session, a second contrast sensitivity function was collected at the mesopic luminance levela condition more closely matching the visual adaptation state of the observers during the performance of the symbol sign legibility assessment procedure. Subjects were then paid $\$ 10.00 / \mathrm{h}$ for their participation. 


\section{RESULTS}

\section{Contrast Sensitivity}

The contrast sensitivity functions collected from each of the age groups under photopic and mesopic luminance conditions are plotted in figures 4 and 5, respectively. An Age (3) by Luminance (2) by Spatial Frequency (5) ANOVA was performed upon the log-transformed contrast sensitivity data. Log transformation was employed to: minimize differences in data variance across spatial frequencies, and reduce nonlinearities introduced by the reciprocal contrast threshold-to-sensitivity operation. The results of the ANOVA revealed a significant effect of Age $[F(2,34)=13.2, p<0.0001]$, as well as a significant Age by Spatial Frequency interaction $[\mathrm{F}(6.12,103.9)=4.45, \mathrm{p}<0.00043$. That is, the general age-related reduction in contrast sensitivity became greater in magnitude as the spatial frequency of the sine-wave target was increased. Simple-effects analyses (summarized in table 17) revealed that significant age-related reductions in contrast sensitivity occurred at all spatial frequencies greater than 1 c/deg. Age group contrasts indicated that the contrast sensitivity of the oldest group was significantly different from both the young and middle-aged groups across this same range of spatial frequencies. Significant differences between the young and middle-aged groups appeared only at $18 \mathrm{c} / \mathrm{deg}$--the highest spatial frequency examined. The main effect of spatial frequency, as is always the case, was highly significant $[\mathrm{F}(3.06,103.9)=153.2, \mathrm{p}<0.0001]$. Contrast sensitivity peaked between 2 to $4 \mathrm{c} / \mathrm{deg}$ and rapidly declined at both higher and lower spatial frequencies. The nature of this spatial frequency trend, as well as the age by spatial frequency interaction effects, are apparent in figures 4 and 5.

The main effect of luminance was also significant $[\mathrm{F}(1,34)=9.53, \mathrm{p}<0.0041$. Reducing the luminance of the target (thus, modifying the adaptation state of the observer) tended to result in decreased contrast sensitivity,--especially for the high spatial frequency targets. However, the statistical evidence for the high spatial frequency locus of the luminance effect was only marginal $[\mathrm{F}(3.73,126.9)=1.93, \mathrm{p}<0.111$. Neither the age by luminance $[\mathrm{F}(2,34)=2.47, \mathrm{p}<0.10]$ interaction nor the age by luminance by spatial frequency $[\mathrm{F}(7.47,126.9)=1.00$, $\mathrm{p}<0.431$ effect was statistically significant. All of the statistical effects involving "repeated measures" factors reported above (and in subsequent sections of this report) have been subjected to the Greenhouse-Geisser correction; hence, the fractional degrees of freedom values reported for the F-tests of such effects. 
Table 17. Age group differences in contrast sensitivity: nighttime legibility (phase 1).

\begin{tabular}{|c|c|c|c|c|}
\hline \multirow[b]{2}{*}{$\begin{array}{l}\text { Spatial } \\
\text { Frequency }\end{array}$} & \multicolumn{4}{|c|}{ Between age group contrasts } \\
\hline & $\begin{array}{l}\frac{\text { Simple age }}{\text { effect }} \\
\underline{F(2,34)}\end{array}$ & $\begin{array}{r}\frac{\text { Young }}{\text { vs. }} \\
\text { Middle-Aged } \\
\end{array}$ & $\begin{array}{l}\frac{\text { Young; }}{\text { vs. }} \\
\text { Older }\end{array}$ & $\begin{array}{c}\frac{\text { Middle-Aged }}{\text { vs. }} \\
\text { Older }\end{array}$ \\
\hline 1 & 2.95 & -- & - & -- \\
\hline 2 & $\begin{array}{r}(\mathrm{NS}) \\
4.27 \\
(\mathrm{P}<0.02)\end{array}$ & $\mathrm{N} \mathrm{S}$ & 0.02 & 0.02 \\
\hline 4 & $\begin{array}{c}5.87 \\
(\mathrm{P}<0.007)\end{array}$ & NS & 0.005 & 0.01 \\
\hline 8 & $\begin{array}{c}10.39 \\
(p<0.001)\end{array}$ & NS & 0.001 & 0.002 \\
\hline 18 & $\begin{array}{c}11.84 \\
(p<0.0001)\end{array}$ & 0.05 & 0.0001 & 0.02 \\
\hline
\end{tabular}

Sign Legibility Distance

The minimum size needed by the observers to meet the legibility criterion for each sign was converted to a legibility distance index using the formula: Legibility distance (in $\mathrm{m}$ ) = (Regulation Sign Size / Threshold Size) x Test Viewing Distance. Average legibility distances for each sign as a function of age are shown in table 18. When the rank-ordered legibility distances of the signs in the current study were compared to those obtained for the same 18 signs in the Daytime Legibility Study, a near-perfect Spearman rank-order correlation was observed $($ Spearman rho $=0.965, \mathrm{p}<0.001)$.

The legibility distance data were analyzed using an Age (3) by Sign (18) ANOVA. Both the main effects of Age $[F(2,36)=34.35, p<0.0001]$ and Sign $[F(5.52,198.9)=206.4, p<$ 0.0001], as well as the Age by Sign interaction $[F(11.1,198.9)=10.2, p<0.0001]$, were highly significant. Simple-effects analyses revealed that the Signs factor was highly significant for all three age groups: Young $[\mathrm{F}(5.22,198.9)=114.2, \mathrm{p}<0.0001]$, Middle-aged $[\mathrm{F}(5.22,198.9)=$ $66.4, \mathrm{p}<0.0001]$, and Older $[\mathrm{F}(5.52,198.9)=35.9, \mathrm{p}<0.0001]$. Similar results were obtained for the simple-effects analyses of the age factor. Highly significant age effects $(p<0.0001)$ were obtained for every sign except RV SANITARY STATION, which was not significant $[\mathrm{F}(2,36)=$ $1.76, \mathrm{p}>0.181$. Simple-simple age effects analyses were performed in order to isolate the specific nature of the age effect (i.e., young vs. middle-aged vs. older) for each of the 18 test signs. The results of these statistical comparisons are summarized in the column labeled "Age Test" on table 18. In general, better legibility distances in the young group (i.e., the "best" signs) were associated with the largest absolute age-related performance decrements. As can be seen in 


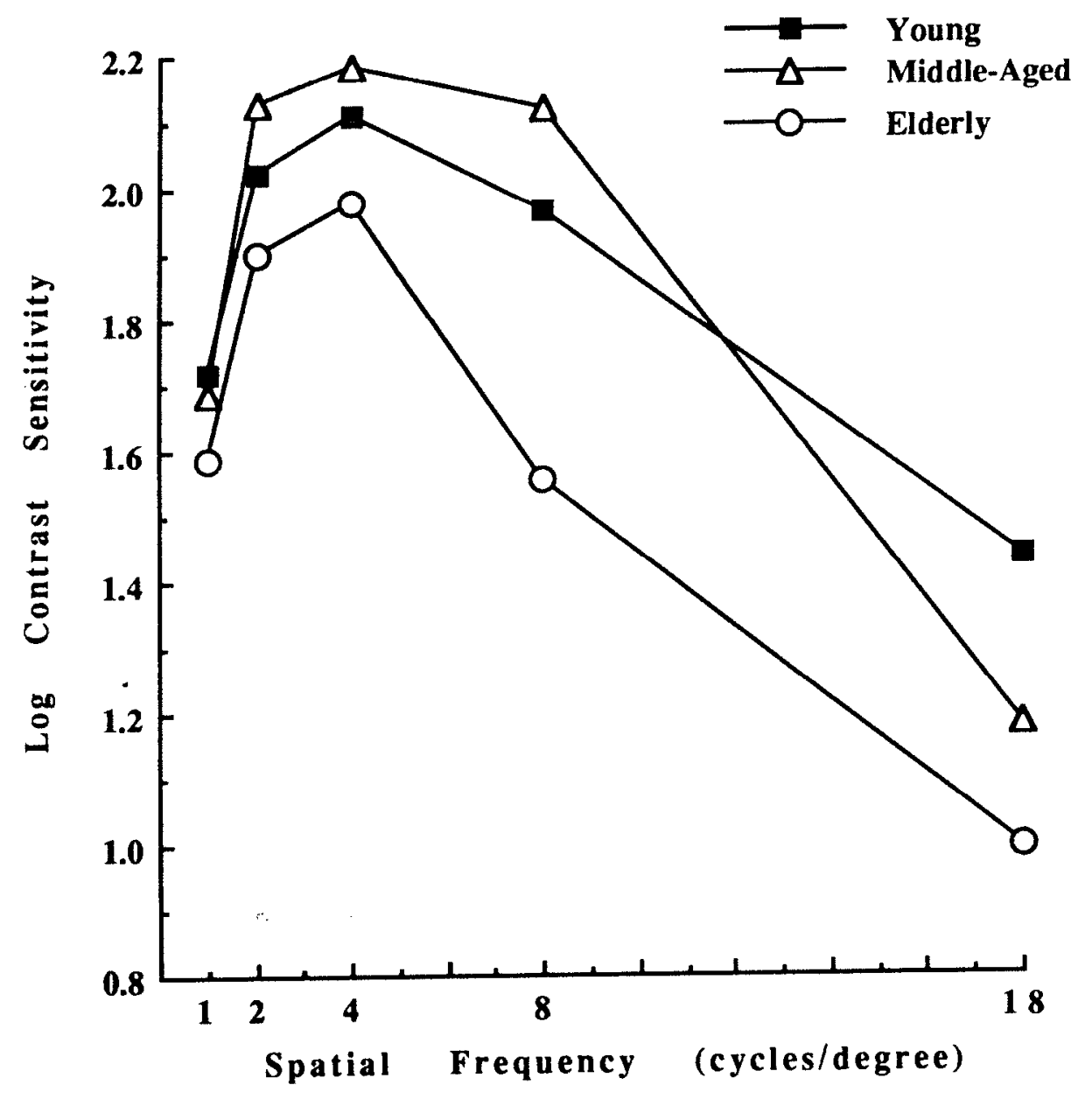

Figure 4. Photopic contrast sensitivity for young, middle-aged, and elderly observers: nighttime legibility (phase 1). 


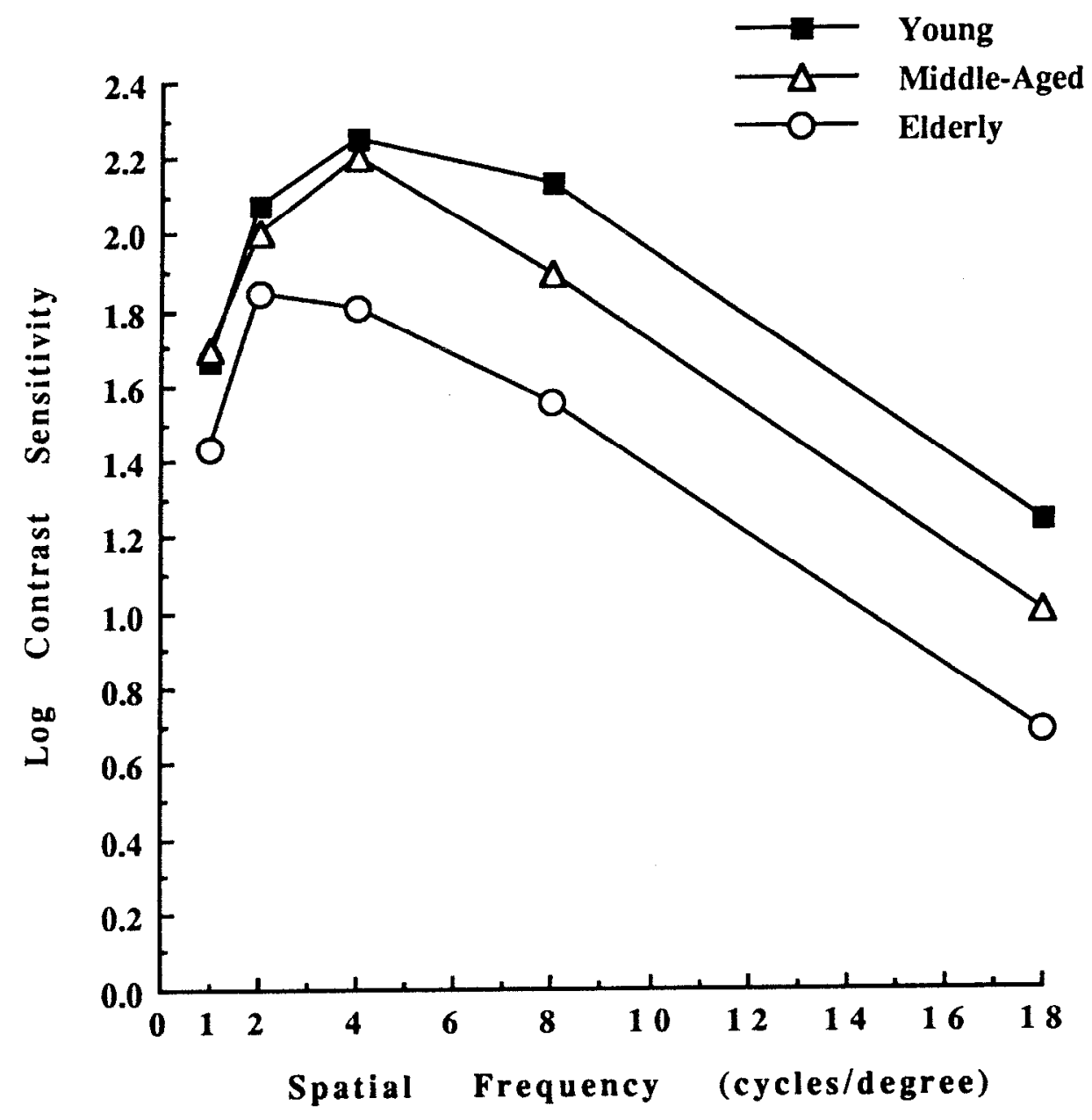

Figure 5. Mesopic contrast sensitivity for young, middle-aged, and elderly observers: nighttime legibility (phase 1). 


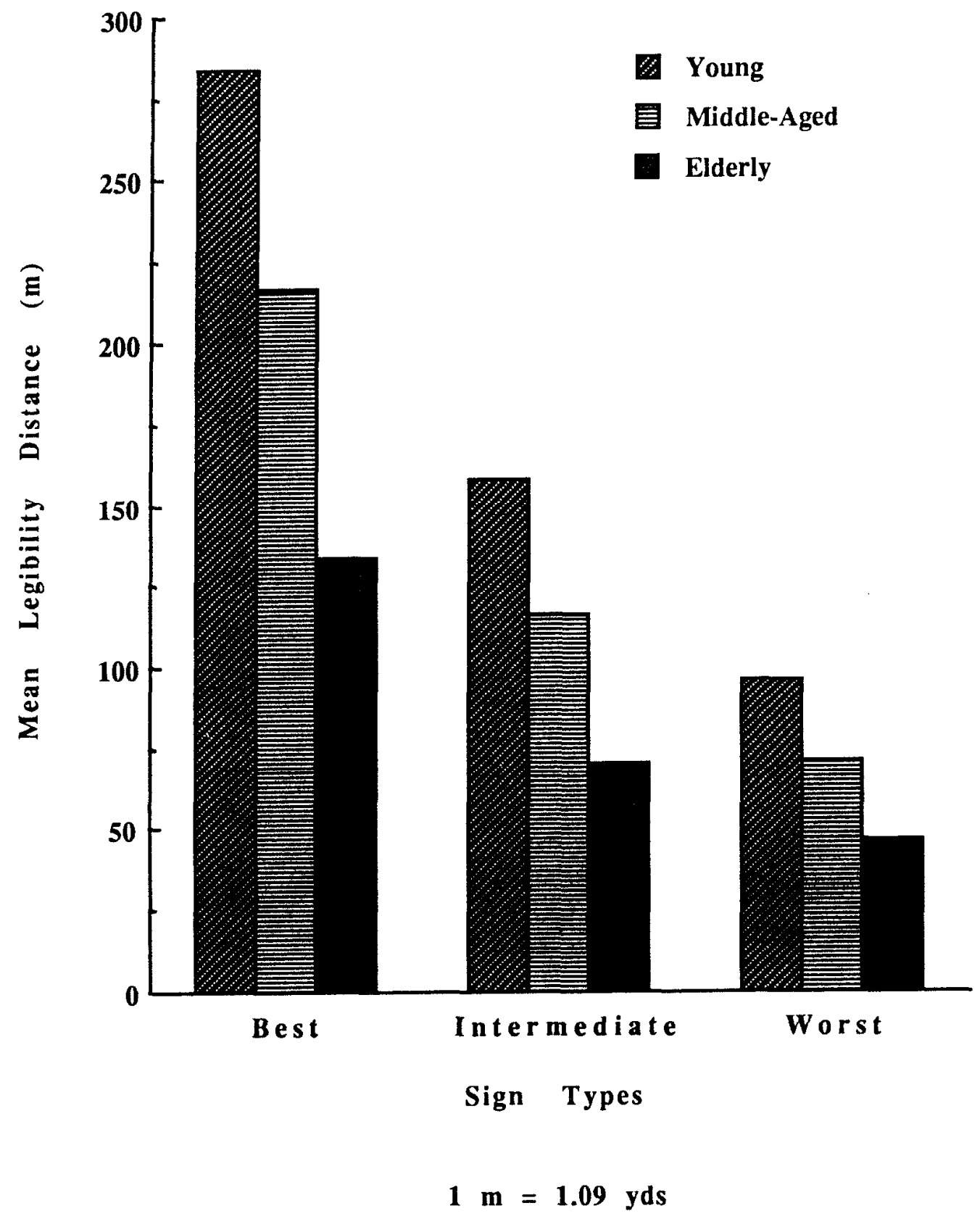

Figure 6. Mean nighttime legibility distances of different sign types as a function of driver age:

nighttime legibility (phase 1). 
figure 6, driver age was closely related to legibility distance for all three types of signs (Best, Intermediate, and Worst), with the distances for the older drivers being about half those required by the young drivers.

\section{Visual Predictors Of Sign Legibility}

Photopic acuity measures and complete contrast sensitivity functions (CSF's) collected under both low photopic and mesopic luminance conditions were available for most of the subjects participating in the current study. Correlational analyses were performed in order to assess the degree to which variations in visual ability, as indexed by acuity and the CSF, might be associated with concomitant variations in overall highway sign legibility performance. In order to simplify the analyses based upon contrast sensitivity, the CSF functions obtained from each subject were fitted with a 3rd order polynomial which was used to parameterize the CSF into three descriptive indices: the half-amplitude low spatial frequency cutoff ("low cutoff"), the highest contrast sensitivity value observed ("peak CS"), and the half-amplitude high spatial frequency cutoff ("high cutoff"). The mean values observed in each age group for each of these CSF parameters, as well as photopic visual acuity, are presented in table 16. Pearson product-moment correlations were calculated between average legibility distance (collapsed across all signs) and each of the following measures of basic visual function: photopic acuity, photopic and mesopic low cutoff frequency, photopic and mesopic log peak CS, and photopic and mesopic high cutoff frequency.

For the overall population of observers (i.e., collapsed across age groups), photopic acuity had the highest correlation with legibility distance $(r=-0.746, p<0.001)$. Photopic low cutoff frequency was not significantly related to legibility distance $(r=0.171)$. However, both photopic $\log$ peak CS $(r=0.472, p<0.01)$ and photopic high cutoff frequency $(r=0.38, p<0.05)$ were significantly related to average legibility distance. Modified Hoteling t-tests revealed that the association between photopic acuity and legibility distance was stronger than the similar relationships observed for photopic log peak CS and high cutoff frequency $(p<0.001)$. Correlational analyses of the CSF parameters derived from the mesopic assessment condition revealed a markedly different pattern of results. All three of the mesopic CSF parameters were significantly related to average legibility distance: low cutoff frequency $(r=0.329, p<0.05), \log$ peak CS $(r=0.71, p<0.001)$, and high cutoff frequency $(r=0.389, p<0.05)$. Modified Hoteling t-tests revealed that mesopic log peak CS had a stronger relationship with legibility distance than either of the CSF cutoff frequencies. Unlike the photopic condition, acuity was not found to be a significantly better predictor of legibility distance than mesopic log peak CS $(\mathrm{t}=$ $0.717, \mathrm{p}>0.5)$."

\section{DISCUSSION}

Strong and systematic differences in legibility distance were found across the 18 symbol signs examined in this study (see table 18). Strong and consistent adult age differences were also superimposed upon this sign effect. Older adults demonstrated significant reductions in legibility distance for every sign except the RV SANITARY STATION sign, which was so difficult to resolve that it represented a "floor effect" in bringing the age group performances together. In addition to these young vs. older performance decrements, marked middle-aged vs. older differences were observed on many of the signs as well (i.e., all except the 
Table 18. Mean legibility distance (m) and standard deviations (SD) for low luminance condition as a function of age: nighttime legibility (phase 1).

\begin{tabular}{|c|c|c|c|c|c|c|c|}
\hline $\begin{array}{c}\mathrm{OU} \\
\mathrm{Rank} \S\end{array}$ & $\begin{array}{c}\mathrm{UC} \\
\mathrm{Rank} \\
\end{array}$ & $\begin{array}{c}\text { Sign } \\
\text { (MUTCD\#) }\end{array}$ & $\begin{array}{l}\text { Age } \\
\underline{\text { Diff }}\end{array}$ & Young & $\begin{array}{l}\text { Middle- } \\
\text { Aged }\end{array}$ & $\underline{\text { Older }}$ & $\begin{array}{c}\text { All } \\
\text { Groups } \\
\end{array}$ \\
\hline 1 & 1 & $\begin{array}{l}\text { Cross Road } \\
\text { (W2-1) }\end{array}$ & $\mathrm{ac}$ & $\begin{array}{l}428.1 \\
(87.9)\end{array}$ & $\begin{array}{c}370.6 \\
(104.4)\end{array}$ & $\begin{array}{l}206.9 \\
(61.1)\end{array}$ & $\begin{array}{l}322.2 \\
(87.7)\end{array}$ \\
\hline 2 & 2 & $\begin{array}{l}\text { Right Curve } \\
\text { (W1-2R) }\end{array}$ & $a b c$ & $\begin{array}{l}355.4 \\
(78.5)\end{array}$ & $\begin{array}{l}264.8 \\
(80.7)\end{array}$ & $\begin{array}{l}181.1 \\
(56.7)\end{array}$ & $\begin{array}{l}258.1 \\
(85.3)\end{array}$ \\
\hline 3 & 5 & $\begin{array}{l}\text { Divided Hwy Ends } \\
\text { (W6-2) }\end{array}$ & $a b c$ & $\begin{array}{l}310.1 \\
(66.5)\end{array}$ & $\begin{array}{l}197.8 \\
(76.9)\end{array}$ & $\begin{array}{l}116.5 \\
(44.9)\end{array}$ & $\begin{array}{l}199.0 \\
(77.5)\end{array}$ \\
\hline 4 & 4 & $\begin{array}{l}\text { Keep Right } \\
\text { (R4-7) }\end{array}$ & $a b$ & $\begin{array}{l}267.8 \\
(93.7)\end{array}$ & $\begin{array}{l}182.6 \\
(67.1)\end{array}$ & $\begin{array}{l}116.4 \\
(57.6)\end{array}$ & $\begin{array}{l}183.0 \\
(65.2)\end{array}$ \\
\hline 5 & 3 & $\begin{array}{l}\text { Hospital } \\
\text { (D9-2) }\end{array}$ & $\mathrm{ac}$ & $\begin{array}{l}260.9 \\
(63.3)\end{array}$ & $\begin{array}{l}206.7 \\
(68.1)\end{array}$ & $\begin{array}{l}139.6 \\
(46.8)\end{array}$ & $\begin{array}{l}197.5 \\
(70.2)\end{array}$ \\
\hline 6 & 7 & $\begin{array}{l}\text { Phone } \\
\text { (D9-1) }\end{array}$ & $a b c$ & $\begin{array}{l}215.3 \\
(47.0)\end{array}$ & $\begin{array}{l}164.6 \\
(55.8)\end{array}$ & $\begin{array}{c}91.3 \\
(41.1)\end{array}$ & $\begin{array}{l}151.1 \\
(51.1)\end{array}$ \\
\hline 7 & 6 & $\begin{array}{l}\text { Advance Flagger } \\
\text { (W20-7a) }\end{array}$ & $\mathrm{ac}$ & $\begin{array}{l}212.8 \\
(71.3)\end{array}$ & $\begin{array}{l}165.5 \\
(64.6)\end{array}$ & $\begin{array}{l}108.2 \\
(37.1)\end{array}$ & $\begin{array}{l}156.6 \\
(67.8)\end{array}$ \\
\hline 8 & 9 & $\begin{array}{l}\text { No U-Turn } \\
\text { (R3-4) }\end{array}$ & $a b c$ & $\begin{array}{l}178.1 \\
(55.4)\end{array}$ & $\begin{array}{l}110.4 \\
(41.8)\end{array}$ & $\begin{array}{c}64.9 \\
(22.4)\end{array}$ & $\begin{array}{l}113.4 \\
(31.7)\end{array}$ \\
\hline 9 & 10 & $\begin{array}{l}\text { No Parking } \\
\text { (R8-3a) }\end{array}$ & $a b c$ & $\begin{array}{l}172.6 \\
(45.1)\end{array}$ & $\begin{array}{l}129.4 \\
(44.5)\end{array}$ & $\begin{array}{c}77.7 \\
(32.9)\end{array}$ & $\begin{array}{l}121.5 \\
(42.1)\end{array}$ \\
\hline 10 & 8 & $\begin{array}{l}\text { Bicycle Crossing } \\
\text { (W11-1) }\end{array}$ & $a b c$ & $\begin{array}{l}157.6 \\
(32.7)\end{array}$ & $\begin{array}{l}119.8 \\
(28.3)\end{array}$ & $\begin{array}{c}81.0 \\
(16.9)\end{array}$ & $\begin{array}{l}116.2 \\
(55.1)\end{array}$ \\
\hline
\end{tabular}

Notes:

OU Rank - Ordinal ranking of signs based upon the performance of the young group

UC Rank - Ordinal ranking of the same signs using Daytime Visibility data collected at the University of Calgary

Age Diff (i.e. post hoc comparison) Legend:
a Young > Older $(p<0.01)$
b Young $>$ Middle-aged $(p<0.01)$
c Middle-aged $>$ Older $(p<0.01)$

$\S$ Spearman Rank-Order Correlation (Rho) analysis of the University of Calgary

daytime the versus the Oakland University nighttime legibility data across signs yielded a value of $0.965(p<0.0001)$.

( ) Standard deviations appear in parentheses 
Table 18. Mean legibility distance (m) and standard deviations (SD) for low luminance condition as a function of age: nighttime legibility (phase 1) (continued).

\begin{tabular}{|c|c|c|c|c|c|c|c|}
\hline $\mathrm{OU}$ & UC & Sign & Age & & Middle & & All \\
\hline Rank $\S$ & $\underline{\mathrm{Rank}}$ & (MUTCD\#) & $\underline{\text { Diff }}$ & Young & Aged & $\underline{\text { Older }}$ & Groups \\
\hline 11 & 11 & $\begin{array}{l}\text { No Trucks } \\
\text { (R5-2) }\end{array}$ & $\mathrm{ac}$ & $\begin{array}{l}111.4 \\
(26.7)\end{array}$ & $\begin{array}{c}93.5 \\
(35.6)\end{array}$ & $\begin{array}{c}51.8 \\
(26.3)\end{array}$ & $\begin{array}{c}82.1 \\
(27.4)\end{array}$ \\
\hline 12 & 14 & $\begin{array}{l}\text { Campfire } \\
\text { (RA-030) }\end{array}$ & $a b c$ & $\begin{array}{l}107.9 \\
(23.7)\end{array}$ & $\begin{array}{l}75.5 \\
(26.5)\end{array}$ & $\begin{array}{c}38.9 \\
(11.9)\end{array}$ & $\begin{array}{c}71.6 \\
(28.9)\end{array}$ \\
\hline 13 & 12 & $\begin{array}{l}\text { Nat Network Route } \\
\text { Prohibited (R14-5) }\end{array}$ & $a b$ & $\begin{array}{c}98.7 \\
(31.2)\end{array}$ & $\begin{array}{l}64.8 \\
(21.0)\end{array}$ & $\begin{array}{c}43.4 \\
(22.5)\end{array}$ & $\begin{array}{c}66.5 \\
(20.7)\end{array}$ \\
\hline 14 & 13 & $\begin{array}{l}\text { Pavement Ends } \\
\text { (W8-3a) }\end{array}$ & $\mathrm{ac}$ & $\begin{array}{l}93.9 \\
(40.4)\end{array}$ & $\begin{array}{c}74.7 \\
(23.2)\end{array}$ & $\begin{array}{c}47.2 \\
(10.9)\end{array}$ & $\begin{array}{c}69.3 \\
(41.1)\end{array}$ \\
\hline 15 & 16 & $\begin{array}{l}\text { Mandatory Seatbelt } \\
\text { (R16-1) }\end{array}$ & $\mathrm{ac}$ & $\begin{array}{c}81.3 \\
(27.1)\end{array}$ & $\begin{array}{c}61.7 \\
(27.6)\end{array}$ & $\begin{array}{l}34.6 \\
(7.28)\end{array}$ & $\begin{array}{c}56.6 \\
(29.4)\end{array}$ \\
\hline 16 & 17 & $\begin{array}{l}\text { Skating (Ice) } \\
\text { (RS-010) }\end{array}$ & $a b$ & $\begin{array}{c}76.9 \\
(26.3)\end{array}$ & $\begin{array}{c}49.4 \\
(14.2)\end{array}$ & $\begin{array}{c}37.5 \\
(11.7)\end{array}$ & $\begin{array}{c}53.0 \\
(29.4)\end{array}$ \\
\hline 17 & 18 & $\begin{array}{l}\text { Ranger Station } \\
\text { (RG-170) }\end{array}$ & $\mathrm{ac}$ & $\begin{array}{c}67.4 \\
(20.5)\end{array}$ & $\begin{array}{c}54.3 \\
(21.7)\end{array}$ & $\begin{array}{l}35.8 \\
(8.85)\end{array}$ & $\begin{array}{c}51.0 \\
(25.4)\end{array}$ \\
\hline 18 & 15 & $\begin{array}{l}\text { RV Sanitary Station } \\
\text { (D9-12) }\end{array}$ & -- & $\begin{array}{l}32.1 \\
(6.49)\end{array}$ & $\begin{array}{c}32.4 \\
(17.1)\end{array}$ & $\begin{array}{l}26.2 \\
(3.45)\end{array}$ & $\begin{array}{l}29.8 \\
(25.1)\end{array}$ \\
\hline
\end{tabular}

KEEP RIGHT, NATIONAL NETWORK ROUTE PROHIBITED, SKATING, and RV SANITARY STATION signs). This is consistent with the pattern of age differences observed in the Daytime Legibility Study. However, unlike the Daytime study, the current examination of legibility under simulated nighttime conditions revealed a large number of signs for which significant young vs. middle-aged differences were observed (viz., RIGHT CURVE, DIVIDED HIGHWAY ENDS, KEEP RIGHT, PHONE, NO U-TURN, NO PARKING, BICYCLE CROSSING, CAMPFIRE, NATIONAL NETWORK ROUTE PROHIBITED, and SKATING). The emergence of such middle-aged legibility decrements across the daytime to nighttime manipulation appears to be the result of at least two concomitant factors: the emergence of ocular opacification in middle age, and the use of a slightly older middle-aged sample in the nighttime legibility study (49.3 vs. 50.8). The significant reduction in contrast sensitivity at the highest spatial frequency observed in the middle-aged group relative to their younger counterparts suggests that optical degradation of the lens was responsible for the emergence of middle-aged reductions in sign legibility at reduced luminance levels.

The rank-ordering of the signs according to legibility distance remained essentially the same across all three age groups. This uniformity of trend demonstrates the reliability of the threshold estimation procedures employed in this and related studies. The near-perfect rank-order correlation observed between the legibility distance of the 18 test signs under nighttime vs. daytime conditions (Spearman's rho $=0.965$ ) suggests that differences in nighttime legibility will yield to straightforward modeling in subsequent research. For example, nighttime legibility of the middle-aged or older, for a given sign, would appear to be predictable on the basis of knowing 
the daytime legibility of the young using a simple multiplicative formula such as:

$$
\text { nighttime legibility older }=\mathrm{k} \times \text { daytime }^{\text {legibility }} \text { young }
$$

Analysis of the contrast sensitivity data revealed a pattern of age differences consistent with the findings of previous research. CSF in the older was significantly depressed at all spatial frequencies above $1 \mathrm{c} / \mathrm{deg}$ (see figures 4 and 5). Middle-aged subjects showed CSF deficits only at $18 \mathrm{c} / \mathrm{deg}$. Luminance reduction from low photopic to mid-mesopic levels resulted in a general decline in contrast sensitivity. This luminance effect tended to be greater at higher spatial frequencies and for older participants--both trends approached statistical significance $(p<0.1)$ but neither exceeded it. Failure to reach significance probably resulted from a combination of "noise" inherent in the rapid CSF data collection procedure (where precision was traded off against time) and the small size of the luminance difference across conditions (viz., 6 vs. 22 $\mathrm{cd} / \mathrm{m}^{2}$ ). However, it should be noted that the CRT-based version of the CSF assessment protocol appeared to yield more discriminative power between subjects than did the VCTS procedure employed in previous studies.

As was the case for the daytime conditions, photopic acuity was found to be a good predictor of overall sign legibility $(\mathrm{r}=-0.746)$. Unlike the previous study, however, "peak CS" was also found to be a powerful predictor of sign legibility--especially when collected under mesopic luminance conditions $(\mathrm{r}=0.71)$. The emergence of a predictive relationship between peak CS and performance probably resulted from the use of the smaller contrast increments in the CRT-based CSF assessment procedure relative to the much more crude "steps" available on the VCTS far chart. The significant improvement in the predictive power of the peak CS measure over the "high cutoff' frequency suggests that it is the degree to which the Fourier spectrum of a sign stimulus "loads" on the peak of the CSF that accounts for its legibility, rather than whether the Fourier power simply falls within vs. outside of the envelope specified by the high spatial frequency rolloff. 


\section{CHAPTER 6: NIGHTTIME LEGIBILITY OF STANDARD SYMBOL HIGHWAY SIGNS IN THE PRESENCE OF PERIPHERAL GLARE}

Visual research with older people indicates that they have increased susceptibility to glare under nighttime conditions, and are slower than younger people to recover from glare. This study examined the effects of peripheral glare on the legibility distances of symbol highway signs as a function of age.

\section{METHOD}

\section{Subjects}

Three age groups of active, community-resident drivers were sampled: a young group (N $=12, \underline{\mathrm{M}}$ age $=19.9$, range 18 to 23$)$, a middle-aged group $(\mathrm{N}=12, \underline{\mathrm{M}}$ age $=47.5$, range 38 to 59$)$, and an older group $(\mathrm{N}=20, \underline{\mathrm{M}}$ age $=70.5$, range 65 to 82 ). The young subjects were recruited from the student population of Oakland University (Rochester, Michigan), and the middle--aged and older subjects were recruited from community service organizations in Auburn Hills and Pontiac, Michigan, respectively. Demographic characteristics of the three age groups are presented in detail in table 19. Visual health of the three age groups, as indexed by photopic acuity, was representative of known population values (see table 20). However, five of the 20 older subjects were excluded from the data analysis because they could not see the highway sign targets in the presence of the glare source.

\section{Stimuli And Apparatus}

Binocular photopic $\left(85 \mathrm{~cd} / \mathrm{m}^{2}\right)$ acuity was measured using a customized Landolt $\mathrm{C}$ chart. This chart divided the acuity range between 6/12 and 6/3 (20/40 and 20/10) into 15 equivalently spaced optotype intervals-yielding a highly precise estimate of spatial resolving power. Contrast sensitivity was assessed using a CRT-based analog of the Vistech VCTS 6500 far chart (used at the University of Calgary laboratory). Contrast sensitivities were measured using a stimulus space-averaged luminance of $22 \mathrm{~cd} / \mathrm{m}^{2}$ at each of 5 spatial frequencies: 1, 2, 4, 8 and 18 cycles per degree of visual angle (c/deg).

The test stimuli consisted of 16 symbol signs selected on the basis of their legibility and comprehension levels in the previous studies. Initial plans called for the use of 18 stimulus signs. However, the PAVEMENT ENDS and RV SANITARY STATION signs were excluded because of "floor effects" obtained with the older participants of the Nighttime Legibility study. That is, these signs could not be seen properly under nighttime conditions. Sign stimuli were presented in their regulation colors using an Apple IIsi computer equipped with a $33 \mathrm{~cm}$ (13-in) RGB monitor. White areas of the screen were maintained at $8.5 \mathrm{~cd} / \mathrm{m}^{2}$. A viewing distance of $5 \mathrm{~m}$ $(16.4 \mathrm{ft})$ was maintained by the use of a head/chin rest.

All legibility measures, except acuity, were collected in the presence of two very bright glare sources located to the left and right of the stimulus display monitors (see figure 7). Each of 
(All units in degrees-of-visual-angle)

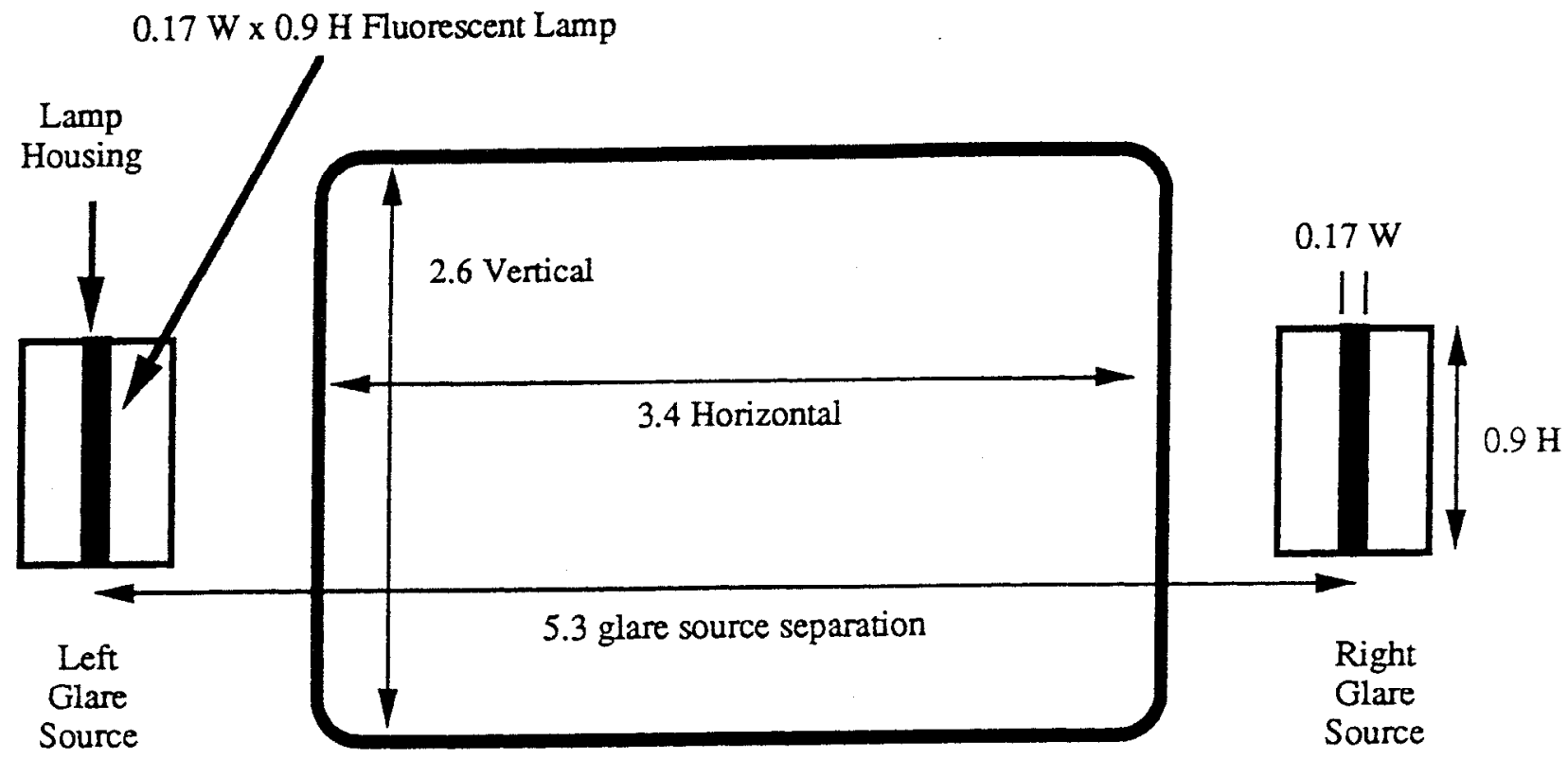
A. Color computer display monitor with peripheral glare sources (used for sign stimuli)

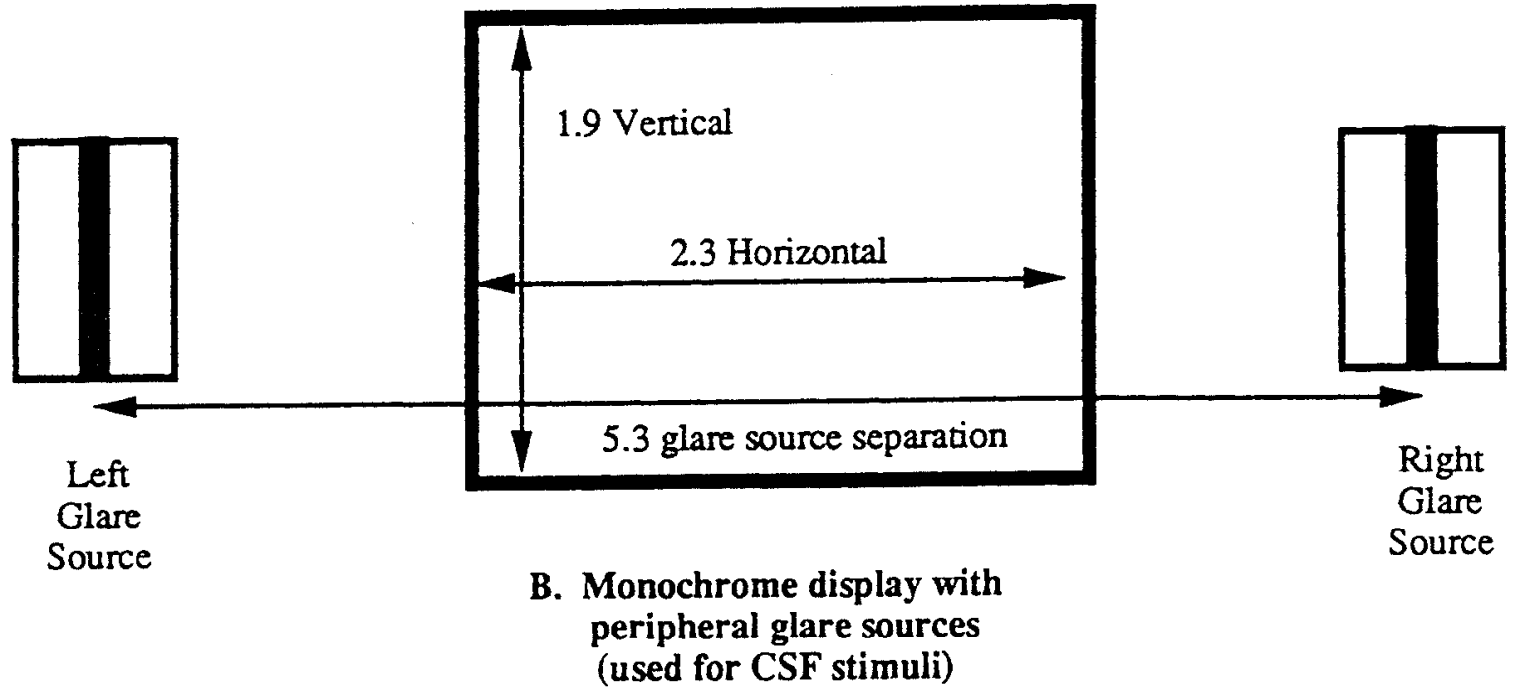

Figure 7. Display apparatus: nighttime-with-glare (phase 1). 
these peripheral glare sources consisted of a fluorescent lamp which subtended a visual angle of 0.17 degrees wide by 0.9 degrees high. Both lamps were mounted in housings which prevented the extra ocular mixing of the glare light with that from the stimulus displays. CRT display equivalent luminance of each glare source was $15000 \mathrm{~cd} / \mathrm{m}^{2}$ (nominal). At the entrance pupil to the eye, the glare sources approximated the intensity of an automobile head lamp viewed at a distance of $30.5 \mathrm{~m}(100 \mathrm{ft})$.

\section{Procedure}

After completion of the informed consent procedure, subjects filled out the standard driving history/status questionnaire used in the previous studies. At this point, their photopic acuity was measured. The laboratory was then darkened, the peripheral glare sources were turned on, and the recognition size/distance thresholds for the practice and test symbol signs were collected using the same procedure detailed in the Method section of the Daytime Legibility study. At the end of this session, the contrast sensitivity function was measured in the presence of the peripheral glare sources, and subjects received an honorarium of $\$ 10 / \mathrm{h}$ for their participation.

Table 19. Participant demographics: night-with-glare legibility (phase 1) (Percentage of Drivers).

\section{Distance driven (miles)}

under 2,000

2,000-4,999

5,000-9,999

10,000-14,999

$15,000-19,999$

20,000 or more
Age groups

\section{$\underline{\text { Middle-aged }}$}

\section{Older}

Young

8

\section{Driving Environment}

rural/sparsely populated

small town

16

suburbs of city

inner city

\section{)}

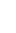


these peripheral glare sources consisted of a fluorescent lamp which subtended a visual angle of 0.17 degrees wide by 0.9 degrees high. Both lamps were mounted in housings which prevented the extra ocular mixing of the glare light with that from the stimulus displays. CRT display equivalent luminance of each glare source was $15000 \mathrm{~cd} / \mathrm{m}^{2}$ (nominal). At the entrance pupil to the eye, the glare sources approximated the intensity of an automobile head lamp viewed at a distance of $30.5 \mathrm{~m}(100 \mathrm{ft})$.

\section{Procedure}

After completion of the informed consent procedure, subjects filled out the standard driving history/status questionnaire used in the previous studies. At this point, their photopic acuity was measured. The laboratory was then darkened, the peripheral glare sources were turned on, and the recognition size/distance thresholds for the practice and test symbol signs were collected using the same procedure detailed in the Method section of the Daytime Legibility study. At the end of this session, the contrast sensitivity function was measured in the presence of the peripheral glare sources, and subjects received an honorarium of $\$ 10 / \mathrm{h}$ for their participation.

Table 19. Participant demographics: night-with-glare legibility (phase 1) (Percentage of Drivers).

$\underline{\text { Age groups }}$

\section{Distance driven}

(miles)

under 2,000

2,000-4,999

5,000-9,999

10,000-14,999

15,000-19,999

20,000 or more

\section{Middle-aged}

Young:

8

\section{Driving Environment}

rural/sparsely populated

small town

suburbs of city

inner city

\section{Type of Vehicle}

passenger car

motor home/RV

motorcycle

light truck/van

heavy truck

bus

$\mathrm{cab}$
16

67

8

100

\section{$\underline{\text { Older }}$}


Table 19. Participant demographics: night-with-glare (phase 1) (Percentage of Drivers) (continued).

\begin{tabular}{|c|c|c|c|}
\hline$\frac{\text { Percentage Driven }}{\underline{\text { at Night }}}$ & $\underline{\text { Young }}$ & $\underline{\text { Middle-aged }}$ & $\underline{\mathrm{OlC}}$ \\
\hline $0-5$ & & & \\
\hline $6-10$ & 8 & 16 & 20 \\
\hline $11-15$ & 25 & 8 & 53 \\
\hline $16-20$ & 35 & 34 & 27 \\
\hline $\begin{array}{l}21-30 \\
31-40\end{array}$ & 8 & 16 & \\
\hline $41-50$ & 16 & 26 & - \\
\hline 51 or more & 8 & & \\
\hline$\frac{\text { Highest Education }}{\text { Level }}$ & & & \\
\hline $1-8$ years & & & 13 \\
\hline some high school & & & 7 \\
\hline high school graduate & 33 & & 42 \\
\hline trade/business college & & 8 & 13 \\
\hline some college or university & 67 & 33 & 25 \\
\hline university degree & & 26 & \\
\hline post graduate education & & 33 & \\
\hline
\end{tabular}

Table 20. Vision assessment parameter as a function of age: nighttime-with-glare legibility (phase 1).

\begin{tabular}{|c|c|c|c|}
\hline \multirow[b]{2}{*}{$\underline{\text { Parameter }}$} & \multicolumn{3}{|c|}{ Age group } \\
\hline & Young & Middle-aged & $\underline{\text { Older }}$ \\
\hline Low Frequency Cutoff (c/deg) & 1.90 & 1.81 & 1.44 \\
\hline Log Peak CS & 2.31 & 2.30 & 1.89 \\
\hline High Frequency Cutoff (c/deg) & 9.55 & 8.80 & 8.02 \\
\hline Acuity (minarc) & $\begin{array}{c}0.635 \\
(\mathrm{SD}=0.12)\end{array}$ & $\begin{array}{c}0.712 \\
(\mathrm{SD}=0.15)\end{array}$ & $\begin{array}{c}1.48 \\
(\mathrm{SD}=0.46)\end{array}$ \\
\hline
\end{tabular}

\section{RESULTS}

\section{Contrast Sensitivity}

The mean contrast sensitivity functions collected from each of the age groups under conditions of glare are plotted in figure 8. An Age (3) by Spatial Frequency (5) ANOVA was performed upon the log-transformed contrast sensitivity data. The results of the ANOVA revealed a significant effect of Age $[\mathrm{F}(2,36)=29.2, \mathrm{p}<0.0001]$, as well as a significant Age by Spatial Frequency interaction $[\mathrm{F}(6.3,113.7)=3.62, \mathrm{p}<0.002]$. That is, the general age-related reduction in contrast sensitivity became greater in magnitude as the spatial frequency of the sine-wave target was increased. Simple-effects analyses (summarized in table 21) revealed that highly significant age-related reductions in contrast sensitivity occurred at all spatial frequencies 


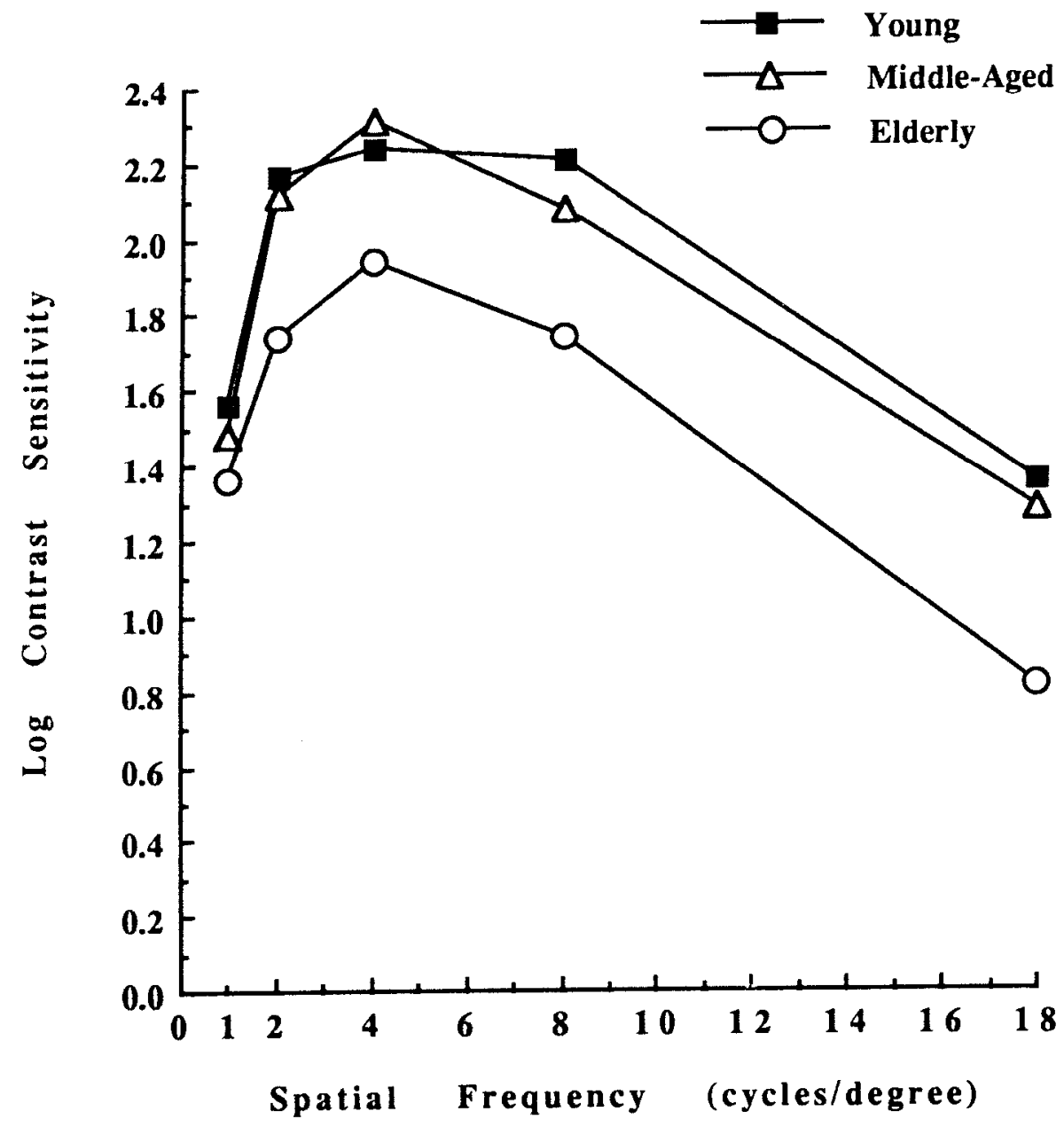

Figure 8. Photopic contrast sensitivity for young, middle-aged, and elderly observers: glare present condition nighttime-with-glare (phase 1). 
greater than $1 \mathrm{c} / \mathrm{deg}$. Between age-group comparisons indicated that the contrast sensitivity of the oldest group was significantly different from both the young and middle-aged groups across this same range of spatial frequencies. No differences in contrast sensitivity were observed between the young and middle-aged groups at any spatial frequency (see table 21). The main effect of Spatial Frequency, as is always the case, was highly significant $[\mathrm{F}(3.2,113.7)=293.1$, $\mathrm{p}<0.0001]$. Contrast sensitivity peaked around $4 \mathrm{c} / \mathrm{deg}$ and rapidly declined at both higher and lower spatial frequencies. The nature of this Spatial Frequency trend, as well as the Age by Spatial Frequency interaction effect, are apparent in figure 8. All of the statistical effects involving repeated measures factors reported above (and in subsequent sections of this report) have been subjected to the Greenhouse-Geisser correction; hence, the fractional degrees of freedom values reported for the F-tests of such effects.

Table 21. Age group differences in contrast sensitivity: nighttime-with-glare legibility (phase 1).

\begin{tabular}{|c|c|c|c|c|}
\hline \multirow[b]{2}{*}{$\begin{array}{l}\text { Spatial } \\
\text { frequency }\end{array}$} & \multirow[b]{2}{*}{$\begin{array}{l}\text { Simple age } \\
\text { effect } \\
F(2,36)\end{array}$} & \multicolumn{3}{|c|}{$\frac{\text { Between age group contrasts }}{(\mathrm{p} \text { levels })}$} \\
\hline & & $\begin{array}{c}\text { Young } \\
\text { vs. } \\
\text { Middle-aged }\end{array}$ & $\begin{array}{c}\text { Young } \\
\text { vs. } \\
\text { Older }\end{array}$ & $\begin{array}{c}\text { Middle-aged } \\
\text { vs. } \\
\text { Older }\end{array}$ \\
\hline 1 & $\begin{array}{l}1.12 \\
(\mathrm{NS})\end{array}$ & --- & --- & --- \\
\hline 2 & $\begin{array}{c}16.79 \\
(\mathrm{P}<0.02)\end{array}$ & NS & 0.0001 & 0.0001 \\
\hline 4 & $\begin{array}{c}15.60 \\
(\mathrm{P}<0.0001)\end{array}$ & $\mathrm{N} \mathrm{S}$ & 0.0002 & 0.0001 \\
\hline 8 & $\begin{array}{c}15.20 \\
(\mathrm{p}<0.0001)\end{array}$ & $\mathrm{N} \quad \mathrm{S}$ & 0.0001 & 0.0001 \\
\hline 18 & $\begin{array}{c}19.01 \\
(\mathrm{p}<0.0001)\end{array}$ & NS & 0.0001 & 0.0001 \\
\hline
\end{tabular}

\section{Sign Legibility Distance}

The minimum size needed by the observers to meet the legibility criterion for each sign was converted to a legibility distance index using the formula: Legibility distance (in $\mathrm{m}$ ) = (Regulation Sign Size/Thresholder Size) x Test Viewing Distance. Average legibility distances for each sign as a function of age enumerated in table 22. When the rank ordered legibility distances of the signs in the current study were compared to those obtained for the same 16 signs in the Daytime Legibility Study, a near-perfect Spearman rank-order correlation was observed $($ Spear-man rho $=0.971, \mathrm{p}<0.001)$.

The legibility distance data were analyzed using an Age (3) by Sign (16) ANOVA. Both the main effects of Age $[F(2,35)=28.4, p<0.0001]$ and $\operatorname{Sign}[F(4.3,150.6)=180.1$, $\mathrm{p}<0.0001]$, as well as the Age by Sign interaction $[\mathrm{F}(8.6,150.6)=8.22, \mathrm{p}<0.0001]$ were highly 
significant. Simple-effects analyses revealed that the Signs factor was highly significant for all three age groups: Young $[\mathrm{F}(4.3,150.6)=83.5, \mathrm{p}<0.0001]$, Middle-aged $[\mathrm{F}(4.3,150.6)=78.6, \mathrm{p}$ $<0.0001]$, and Older $[\mathrm{F}(4.3,150.6)=28.8, \mathrm{p}<0.0001]$. Similar results were obtained for the simple-effects analyses of the Age factor. Highly significant age effects $(\mathrm{p}<0.01)$ were obtained for every sign except SKATING which was not significant $[\mathrm{F}(2,35)=1.12, \mathrm{p}>0.31$.

Simple-simple age effects analyses were performed in order to isolate the specific nature of the age-group effect (i.e., young vs. middle-aged vs. older) for each of the 16 test signs. The results of these statistical comparisons are summarized in the column labeled "Age Diff" on table 22. In general, better legibility distances in the young group (i.e., the "best" signs) were associated with the largest absolute age-related performance decrements.

The legibility distance data collected under conditions of glare in the present study were compared to similar measures observed in the previous study (Nighttime Legibility Without Glare). This comparison clearly indicates that the criterion legibility distance of symbol highway signs was not influenced by the introduction of the bright, peripheral glare sources employed in the current study. Because the introduction of a glare source would be expected to have its greatest deleterious impact upon older drivers, a similar cross-study comparison (i.e., glare vs. no-glare) was made using only the legibility data collected from the respective older age groups. Again, no consistent deleterious effects of glare can be observed. Statistical analysis of the data confirm this conclusion (viz., the test of the glare vs. no-glare condition yielded an $\mathrm{F}(1,18)=$ $2.91, \mathrm{p}<0.10$; while the test of the glare vs. no glare by 16-sign interaction yielded an $\mathrm{F}(5.3$, $95.5)=1.91, \mathrm{p}<0.10$. As with the preceding study, older drivers had shorter legibility distances than did the other two age groups for all three types of signs (see figure 9), however, the differences between older and young drivers were not as marked as in the no-glare condition.

\section{Visual Predictors Of Sign Legibility}

Correlational analyses were performed in order to assess the degree to which variations in visual ability, as indexed by acuity and the contrast sensitivity function (CSF), might be associated with concomitant variations in overall highway sign legibility. The mean values observed in each age group for each of these CSF parameters, as well as photopic visual acuity are presented in table 20. Pearson product-moment correlations were calculated between average legibility distance (collapsed across all signs) and each of the following measures of assessed visual function: acuity, low cutoff frequency, log peak CS, and high cutoff frequency. A summary of these correlational analyses is presented in table 23 .

For the overall population of observers (i.e., collapsed across age groups), acuity had the highest correlation with legibility distance $(r=-0.822, \mathrm{p}<0.001)$. Statistically significant, yet weaker, correlations were observed between overall sign legibility distance and each of the parameterized CSF measures: low cutoff frequency $(\mathrm{r}=0.391, \mathrm{p}<0.01), \log$ peak contrast sensitivity $(r=0.695, p<0.001)$, and high cutoff frequency $(r=0.429, p<0.006)$. Modified Hoteling t-tests revealed that the association between acuity and legibility distance was significantly stronger than the similar relationships observed for the low cutoff frequency $(\mathrm{t}=$ $4.15, \mathrm{p}<0.001), \log$ peak contrast sensitivity $(\mathrm{t}=2.97, \mathrm{p}<0.01)$, and the high cutoff frequency $(\mathrm{t}$ $=4.21, \mathrm{p}<0.001)$. Among the CSF parameters, log peak contrast sensitivity was more strongly related to overall legibility distance than was the low cutoff frequency $(\mathrm{t}=3.26, \mathrm{p}<0.01)$ but not the high cutoff frequency $(\mathrm{t}=1.79, \mathrm{p}<0.10)$. 
Table 22. Highway signs ranked on legibility distance (m) for low luminance condition: nighttime-with-glare legibility (phase 1).

\begin{tabular}{|c|c|c|c|c|c|c|c|}
\hline $\mathrm{OU}$ & UC & Sign & Age & \multicolumn{3}{|c|}{ Middle- } & All \\
\hline$\underline{\mathrm{Rank}} \S$ & $\underline{\mathrm{Rank}}$ & (MUTCD\#) & $\underline{\text { Diff }}$ & Young & $\underline{\text { Aged }}$ & Older & Groups \\
\hline 1 & 1 & $\begin{array}{l}\text { Cross Road } \\
(\mathrm{W} 2-1)\end{array}$ & $a b c$ & $\begin{array}{l}364.0 \\
(86.1)\end{array}$ & $\begin{array}{c}412.4 \\
(118.3)\end{array}$ & $\begin{array}{l}216.8 \\
(92.9)\end{array}$ & $\begin{array}{c}325.0 \\
(129.6)\end{array}$ \\
\hline 2 & 2 & $\begin{array}{l}\text { Right Curve } \\
\text { (W1-2R) }\end{array}$ & $\mathrm{a}$ & $\begin{array}{l}362.4 \\
(62.9)\end{array}$ & $\begin{array}{l}274.9 \\
(41.9)\end{array}$ & $\begin{array}{l}172.3 \\
(39.6)\end{array}$ & $\begin{array}{l}264.7 \\
(92.8)\end{array}$ \\
\hline 3 & 5 & $\begin{array}{l}\text { Divided Hwy Ends } \\
\text { (W6-2) }\end{array}$ & ac & $\begin{array}{l}236.6 \\
(60.4)\end{array}$ & $\begin{array}{l}207 . \mathrm{I} \\
(40.8)\end{array}$ & $\begin{array}{l}126.2 \\
(41.3)\end{array}$ & $\begin{array}{l}186.6 \\
(67.1)\end{array}$ \\
\hline 4 & 4 & $\begin{array}{l}\text { Keep Right } \\
\text { (R4-7) }\end{array}$ & $\mathrm{ac}$ & $\begin{array}{l}234.5 \\
(83.1)\end{array}$ & $\begin{array}{l}203.2 \\
(48.9)\end{array}$ & $\begin{array}{l}137.8 \\
(63.2)\end{array}$ & $\begin{array}{l}189.0 \\
(76.8)\end{array}$ \\
\hline 5 & 3 & $\begin{array}{l}\text { Hospital } \\
\text { (D9-2) }\end{array}$ & $\mathrm{a}$ & $\begin{array}{l}226.9 \\
(50.6)\end{array}$ & $\begin{array}{l}208.3 \\
(26.3)\end{array}$ & $\begin{array}{l}156.3 \\
(56.6)\end{array}$ & $\begin{array}{l}195.0 \\
(55.2)\end{array}$ \\
\hline 6 & 7 & $\begin{array}{l}\text { Phone } \\
\text { (D9-1) }\end{array}$ & $a b c$ & $\begin{array}{l}199.7 \\
(47.6)\end{array}$ & $\begin{array}{l}176.0 \\
(63.0)\end{array}$ & $\begin{array}{l}101.4 \\
(40.3)\end{array}$ & $\begin{array}{l}156.0 \\
(65.6)\end{array}$ \\
\hline 7 & 6 & $\begin{array}{l}\text { Advance Flagger } \\
(\mathrm{W} 20-7 \mathrm{a})\end{array}$ & $\mathrm{ac}$ & $\begin{array}{l}168.2 \\
(61.9)\end{array}$ & $\begin{array}{l}161.1 \\
(40.1)\end{array}$ & $\begin{array}{l}104.9 \\
(44.1)\end{array}$ & $\begin{array}{l}142.6 \\
(56.2)\end{array}$ \\
\hline 8 & 9 & $\begin{array}{l}\text { No U-Turn } \\
\text { (R3-4) }\end{array}$ & $\mathrm{ac}$ & $\begin{array}{l}167.7 \\
(40.3)\end{array}$ & $\begin{array}{l}121.7 \\
(38.9)\end{array}$ & $\begin{array}{c}70.3 \\
(20.2)\end{array}$ & $\begin{array}{l}117.3 \\
(52.4)\end{array}$ \\
\hline 9 & 10 & $\begin{array}{l}\text { No Parking } \\
\text { (R8-3a) }\end{array}$ & $a b c$ & $\begin{array}{l}154.1 \\
(33.8)\end{array}$ & $\begin{array}{l}125.6 \\
(34.9)\end{array}$ & $\begin{array}{c}84.1 \\
(30.5)\end{array}$ & $\begin{array}{l}119.3 \\
(43.5)\end{array}$ \\
\hline 10 & 8 & $\begin{array}{l}\text { Bicycle Crossing } \\
\text { (W11-1) }\end{array}$ & $a b c$ & $\begin{array}{l}133.9 \\
(22.2)\end{array}$ & $\begin{array}{l}110.9 \\
(15.2)\end{array}$ & $\begin{array}{c}79.5 \\
(20.3)\end{array}$ & $\begin{array}{l}106.6 \\
(29.8)\end{array}$ \\
\hline 11 & 11 & $\begin{array}{l}\text { No Trucks } \\
\text { (R5-2) }\end{array}$ & $\mathrm{ac}$ & $\begin{array}{c}95.1 \\
(29.8)\end{array}$ & $\begin{array}{l}106.7 \\
(22.7)\end{array}$ & $\begin{array}{c}73.5 \\
(28.2)\end{array}$ & $\begin{array}{c}90.8 \\
(30.0)\end{array}$ \\
\hline
\end{tabular}

Notes:

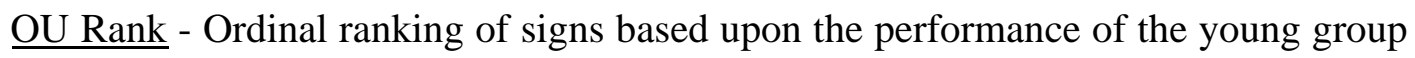

UC Rank - Ordinal ranking of the same signs using Daytime Legibility data collected at the University of Calgary.

Age Diff (i.e. post hoc comparison) Legend:

a Young > Older $(\mathrm{p}<0.01)$

b Young $>$ Middle-aged $(\mathrm{p}<0.01)$

c Middle-aged $>$ Older $(\mathrm{p}<0.01)$

$\S$ Spearman Rank-Order Correlation (Rho) analysis of the University of Calgary daytime versus the Oakland University glare legibility data across signs yielded a value of 0.971 ( $\mathrm{p}<$ $0.0001)$. 
Table 22. Highway signs ranked on legibility distance $(m)$ for low luminance condition: nighttime-with-glare legibility (phase 1) (continued).

\begin{tabular}{|c|c|c|c|c|c|c|c|}
\hline $\mathrm{OU}$ & $\mathrm{UC}$ & Sign & Age & \multicolumn{3}{|c|}{ Middle- } & All \\
\hline$\underline{\mathrm{Rank}} \S$ & $\underline{\mathrm{Rank}}$ & (MUTCD\#) & $\underline{\text { Diff }}$ & Young & Aged & Older & Groups \\
\hline 12 & 13 & $\begin{array}{c}\text { Campfire } \\
(\mathrm{RA}-030)\end{array}$ & $\mathrm{ac}$ & $\begin{array}{c}82.7 \\
(24.6)\end{array}$ & $\begin{array}{c}72.8 \\
(15.4)\end{array}$ & $\begin{array}{c}44.3 \\
(14.7)\end{array}$ & $\begin{array}{c}65.4 \\
(24.7)\end{array}$ \\
\hline 13 & 12 & $\begin{array}{l}\text { Nat Network Route } \\
\text { Prohibited (R14-5) }\end{array}$ & & $\begin{array}{c}80.1 \\
(26.8)\end{array}$ & $\begin{array}{c}87.4 \\
(23.5)\end{array}$ & $\begin{array}{c}43.8 \\
(15.4)\end{array}$ & $\begin{array}{c}69.0 \\
(29.2)\end{array}$ \\
\hline 14 & 14 & $\begin{array}{l}\text { Mandatory Seatbelt } \\
\text { (R16-1) }\end{array}$ & $\mathrm{a}$ & $\begin{array}{l}73.4 \\
(21.1)\end{array}$ & $\begin{array}{c}58.2 \\
(16.7)\end{array}$ & $\begin{array}{c}40.7 \\
(15.2)\end{array}$ & $\begin{array}{l}56.5 \\
(22.0)\end{array}$ \\
\hline 15 & 16 & $\begin{array}{l}\text { Ranger Station } \\
(\mathrm{RG}-170)\end{array}$ & $\mathrm{ac}$ & $\begin{array}{c}59.1 \\
(15.6)\end{array}$ & $\begin{array}{l}52.1 \\
(9.2)\end{array}$ & $\begin{array}{c}39.6 \\
(12.8)\end{array}$ & $\begin{array}{c}49.7 \\
(15.0)\end{array}$ \\
\hline 16 & 15 & $\begin{array}{l}\text { Skating (Ice) } \\
(\mathrm{RS}-010)\end{array}$ & & $\begin{array}{l}57.4 \\
(29.7)\end{array}$ & $\begin{array}{c}57.3 \\
(20.7)\end{array}$ & $\begin{array}{c}44.5 \\
(25.1)\end{array}$ & $\begin{array}{c}52.6 \\
(25.5)\end{array}$ \\
\hline
\end{tabular}

These correlational analyses were also performed separately for each of the respective age groups. Reference to table 23 reveals that only acuity was significantly related to sign legibility distance in the young group, while none of the visual assessment parameters yielded significant correlations for the middle-aged group. Examination of the data suggests that a lack of variation in the visual assessment measures collected within these groups (i.e., the "restricted range problem") may have been responsible for the small correlations observed. The increased within group variation of the visual assessment measures obtained for the older group was accompanied by significant correlations for acuity, as well as log peak contrast sensitivity and the high cutoff frequency.

Table 23. Correlational relationships (r) between visual assessment parameters and legibility distance: nighttime-with-glare legibility (phase 1).

Age group

$\underline{\text { Parameter }}$

Low Frequency

Cutoff (c/deg)

Log Peak CS

High Frequency

Cutoff (c/deg)

Acuity

$\mathrm{NS}=$ not significant
Total population

0.391

$(\mathrm{p}<.01)$

0.695

$(\mathrm{p}<.001)$

0.429

$(\mathrm{p}<.006)$

$-0.822$

$(\mathrm{p}<.001)$
Young

0.225

NS

0.038

NS

0.219

NS

$-0.723$

$(\mathrm{p}<.008)$ $\underline{\text { Middle-aged }}$

$\underline{\text { Older }}$

-0.056
NS

0.412

NS

0.424

NS

0.503

$(\mathrm{p}<.05)$

0.641

NS

$(\mathrm{p}<.01)$

$-0.348$

NS
$-0.643$

$(\mathrm{p}<.01)$ 


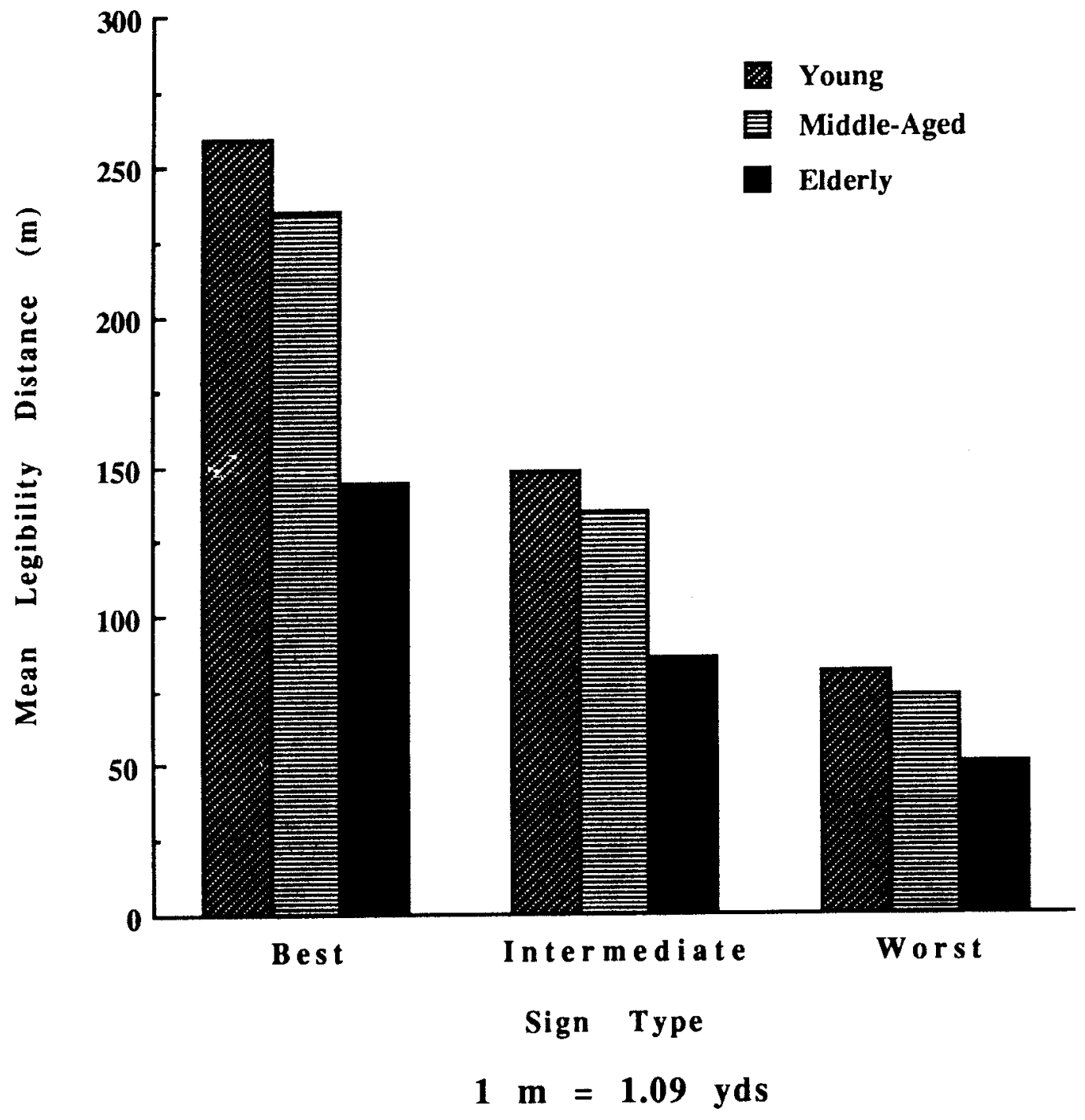

Figure 9. Mean nighttime legibility distances of different sign types as a function of driver age nighttime-with-glare (phase 1). 


\section{DISCUSSION}

Strong and systematic differences in legibility distance were found across the 16 symbol signs examined in this study. Strong and consistent adult age differences were also superimposed upon this sign effect. The nature and magnitude of the loss in legibility distance observed for the oldest participants in the presence of glare mirrored that observed without glare. In fact, the major conclusion to be reached by the current study is that a general glare factor does not need to be considered in the optimization of symbol highway sign legibility. The same rank-ordering of legibility across signs has been shown to holder under widely varying viewing conditions: daytime, nighttime, and nighttime with intense static glare. There are some caveats, however, regarding the unconditional acceptance of the findings of this experiment.

The first concern involves the intensity and the positional placement of the glare sources used in the current investigation. The selection of the glare source intensity involved a tradeoff between brightness levels which would ensure the detection of an age difference (an extremely intense source) versus a glare level which was more representative of the conditions likely to be encountered on the highway at night. In pilot studies not reported here, the Oakland Vision Laboratory evaluated several levels of glare - ranging from 0.5 to 4 times the intensity which was finally selected. At the most intense levels, none of the older observers could detect the presence of the dim highway sign stimuli. At these same levels, however, the youngest observers demonstrated little or no impairment in performance. Subjects of all ages complained about excessive fatigue and discomfort at these higher glare levels. On the basis of this pilot work, a glare source was selected which was representative of glare intensities encountered from oncoming traffic while driving at night, and not so intense as to limit the duration of the experimental session. Dual peripheral sources were chosen because they enabled the delivery of more luminous flux with less observer discomfort, while their 2.6 degree separation from the center of the target stimulus approximated the position of the most likely sources of deleterious glare to be experienced during nighttime reading of highway signs.

Despite the care taken in selecting the intensity of the experimental glare source, an important problem emerged during the progress of the study. That is, 5 of the 44 participants were unable to identify at least half of the symbol signs when presented at their largest possible size on the CRT display. All of these "failing" participants were aged 65 or older. It is likely that these 5 older observers suffered from advanced cataracts or related visual pathology; however, any such supposition must remain speculative, as none of the participants received ophthalmic examinations. Since 5 of the 20 older participants were excluded from the data analyses, interpretation of the results in terms of its "representativeness" becomes a potential problem. However, a less pessimistic interpretation is that when pathological glare problems do exist (e.g., advanced cataract) the effects upon nighttime legibility are so profound as to obviate the role of design optimization. Future research efforts will need to employ more rigorous ophthalmic screening procedures (such as the collection of lens opacification and/or scatter indices) and provide for alternative glare sources to evaluate those older participants with pathological glare problems.

One final warning about the interpretation of these results is in order. A post hoc examination of the data collection sheets revealed that the participants, especially the older ones, 
tended to demonstrate especially poor legibility distances for the first 2 to 4 signs presented during the experimental protocol. However, since the order of the sign stimuli was completely randomized across subjects such an early "adaptation effect" was unrecoverable from the data. However, if early adaptation to the deleterious effects of glare was occurring, the problems of highway related glare may have been underestimated in the current investigation. That is, the type of glare encountered upon the highway tends to be momentary or "transient," allowing little opportunity for adaptation effects to accrue. However, the present investigation used a constant or "static" exposure to a glare source whose deleterious effects may have been at least partially mitigated by the process(es) of adaptation. Although the likelihood that such adaptation processes significantly altered the effects observed remains remote, previous research indicating that recovery to transient glare slows with advanced age suggests that future efforts aimed at evaluating age differences in highway legibility at night evaluate the effects of glare presented under transient as well as static conditions. 


\section{CHAPTER 7: DAYTIME GLANCE LEGIBILITY AND REACTION TIME FOR SYMBOL HIGHWAY SIGNS AS A FUNCTION OF DRIVER AGE}

At times it may be necessary for a driver to respond quickly and effectively to a sign that can be seen only briefly (e.g., at high speeds or when obscured by another vehicle or vegetation). With advancing age, however, there are well-documented declines in the speed of most information processing and psychomotor functions. Thus older drivers could be more adversely affected by briefly presented signs, especially if their legibility with a single glance is low. The major goals of this study were to: assess age differences in glance legibility of and reaction time to a test set of standard U. S. symbol highway signs, and determine to what degree differences in either measure are associated with age declines in spatial vision.

Although the underlying mechanisms are not fully understood, there are significant losses with aging in most visual functions, including visual acuity, contrast sensitivity, and information processing speed, as well as in psychomotor functioning. Little is known however, about the degree to which such changes affect the ability of older drivers to identify the message of brieflyseen highway signs, or to respond quickly and appropriately to them.

Previous research has indicated that the glance legibility of symbol signs is generally superior to that of word-message signs. When Dewar and Swanson, asked drivers to identify briefly-presented symbol, word, and symbol/word signs, symbol signs were the most easily identified. ${ }^{(21)}$ Similarly, Avant, Brewer, and Thieman reported that drivers made fewer errors in identifying symbol signs than word signs when they were presented briefly in a tachistoscope. ${ }^{(44)}$ Although recent research has shown that symbol signs provide middle-aged and older, as well as young drivers, with the same legibility distance advantages over word signs, the effects of advanced age on glance legibility have yet to be investigated. ${ }^{(19,57)}$ It is also unclear to what degree age differences in sign effectiveness might vary as a function of the characteristics of the symbol sign itself.

In the present study, 18 standard symbol signs that varied greatly in their tested legibility distance and ease of comprehension were compared in regard to the ability of young, middleaged, and older drivers to identify them when they were briefly presented (Glance Legibility). In addition, the latency with which these drivers could indicate nonverbally whether or not a symbol sign corresponded to the name of the sign that preceded it (Reaction Time) was measured.

\section{METHOD}

\section{Subjects}

Three volunteer age groups of active, licensed, community-resident drivers participated in the study: a young group (6 men and 6 women), $\underline{\mathrm{M}}$ age $=24.8$ years (range 19 to 33), a middleaged group (6 men and 6 women), $\underline{\mathrm{M}}$ age $=48.3$ years (range 39 to 60), and an older group ( 9 men and 9 women), $\underline{\mathrm{M}}$ age $=66.9$ years (range 61 to 75). The mean number of years of formal education was 14.5 (range 12 to 18 ) for the young, 15.3 (range 13 to 22) for the middle-aged, and 14.8 (range 9 to 20 ) for the older. None of the age differences in education were significant ( $\mathrm{p}=$

.79). Overall, the participants were in good general health although older drivers were somewhat more likely than younger ones to report some chronic illness or complaint: young, 1 of 12; middle-aged, 2 of 12; and older, 4 of 18. Middle-aged (4 of 12) and older (7 of 18) participants 
were also more likely than the young ( 0 of 12$)$ to take one or more medications on a regular basis. Self-reported visual health of the participants appeared to decline somewhat with age. Of the 12 young drivers, 11 reported no visual problems that were not correctable by refraction and one reported having amblyopia (lazy eye); among the 12 middle-aged participants, 11 had no visual problems and 1 report of amblyopia; and among the 18 older drivers, there were 11 reports of no visual problems, 3 reports of cataracts, 1 report of color blindness, 1 report of night blindness, 1 report of an overly large pupil, and 1 report of both color blindness and amblyopia (see table 24). Because the age-related loss in accommodation reserve (i.e., presbyopia) could disadvantage the older participants in the near $83 \mathrm{~cm}$ (32.7 in) test distance, all participants were refracted to their best acuity. The resulting $\mathrm{M}$ binocular test-distance photopic Landolt- $\mathrm{C}$ acuity levels were: 0.75 minarc (range 0.70 to 1.03 ) for the young, 0.75 minarc (range .70 to .90) for the middle-aged, and 0.97 minarc (range 0.70 to 0.97 ) for the older participants [ 1.0 minarc $=6 / 6$ (20/20)]. Although they were not significantly different from each other, the best-corrected acuity levels of both the young $(\mathrm{p}<.01)$ and the middle-aged drivers $(\mathrm{p}<.01)$ exceeded those of the older.

Table 24. Participant demographics: glance legibility and reaction time.

Average number of years driving experience as a function of age and gender

Age Group

\begin{tabular}{lccc} 
Gender & Young & Middle-Aged & Older \\
\cline { 2 - 2 } & 11.7 & 29.5 & 43.8 \\
Females & 5.2 & 26.2 & 37.0 \\
Total & 8.4 & 27.8 & 41.7
\end{tabular}

Average number of years held a driver's license

Age Group

Years $\frac{\text { Young }}{8.2} \quad \frac{\text { Middle-Aged }}{27.3} \quad \frac{\text { Older }}{41.9}$

Percentage of drivers by distance driven last 12 months as a function of age

Distance Driven

$\underline{\text { (miles) }}$

under 2,000

$2,000-4,999$

$5,000-9,999$

$10,000-14,999$

$15,000-19,999$

20,000 or more
Age Group
Young

41.6

25.0

16.7
Middle-Aged

25.0

33.3

8.3

25.0

8.3 $\underline{\text { Older }}$

11.1

55.6

16.7

11.1 
Table 24. Participant demographics: glance legibility and reaction time (continued).

Percentage of drivers by driving environment as a function of age

Driving Environment

Rural/sparsely populated Age Group

Small town

Middle-Age

\begin{tabular}{ccc} 
Young & Middle-Age & Older \\
\hline 8.3 & & 5.5 \\
25.0 & 58.3 & 27.8 \\
50.0 & 41.7 & 61.1 \\
16.7 & - & 5.6
\end{tabular}

High-density urban

Highway

16.7

5.6

Percentage of drivers by night driving as a function of age

Percentage

Night Driving

$0-5$

6- 10

$11-15$

16-20

21-30

31-40

41-50

51 or more

Percentage

$\underline{\text { Rush Hour }}$

0 - 5

6- 10

11- 15

16-20

21-30

$31-40$

41-50

51 or more
Age Group

Young

8.3

16.7

41.7

8.3

16.7

8.3

$-$
Middle-Aged

8.3

50.0

16.7

16.7

8.3

8.3

8.3 $\underline{\text { Older }}$

66.7

5.6

22.2

11.1

11.1

5.6

Percentage done on the highway as a function of age

Percentage

Highway

0 - 5

6- 10

$11-15$

16-20

21-30

31-40

$41-50$

51 or more $\frac{\text { Young }}{25.0}$

25.0

8.3

25.0

Young

16.7

25.0

8.3

8.3

16.7

8.3

16.7

\section{Age Group}

$\underline{\text { Middle-Aged }}$

16.7

16.7

8.3

25.0

$\underline{\text { Older }}$

50.0

5.6

22.2

11.1

$8.3 \quad 5.6$

8.3

16.7

5.6 Age Group Middle-Aged

Older

8.3

16.6

16.7

22.2

16.6

11.1

11.1

16.7

11.1

8.3

5.6

16.7

5.6 
Table 24. Participant demographics: glance legibility and reaction time (continued).

\begin{tabular}{|c|c|c|c|}
\hline \multirow[b]{2}{*}{ Type of Vehicle } & \multicolumn{3}{|c|}{ Age Group } \\
\hline & Young & Middle-Aged & $\underline{\text { Older }}$ \\
\hline Passenger Car & 83.3 & 66.7 & 83.3 \\
\hline Motor home/RV & & & 5.6 \\
\hline Motorcycle & & & \\
\hline Light Truck/Van & 16.7 & 33.3 & 11.1 \\
\hline $\begin{array}{l}\text { Heavy Truck } \\
\text { Bus }\end{array}$ & & & \\
\hline
\end{tabular}

Stimuli And Apparatus

Participants' best Landolt $\mathrm{C}$ acuity was assessed at a luminance of $100 \mathrm{~cd} / \mathrm{m}^{2}$. Contrast sensitivity was measured at spatial frequencies of 1.5, 3.0, 6.0, 12.0, and $18.0 \mathrm{c} / \mathrm{deg}$ using the Vistech VCTS 6000 Near chart (Form A) at its recommended viewing distance of $46 \mathrm{~cm}$ (18 in) and a luminance of $135 \mathrm{~cd} / \mathrm{m}^{*}$. To allow a comparison between acuity and contrast sensitivity in their relationships with glance legibility and reaction time under comparable conditions, acuity and contrast sensitivity were both assessed using the participant's best optical corrections at each of the two respective test distances. Refractions were carried out using an American Optical Master Phoropter, and optical corrections were implemented with an R.H. Burton trial lens set with a trial frame or lens clip; luminance levels were measured using a Minolta LS-110 spot photometer.

The test stimuli were 18 regulation symbol signs from the MUTCD selected from among the 85 symbol signs whose daytime legibility and comprehensibility for drivers of different age had already been evaluated in the previous studies. ${ }^{(3)}$ To assure that signs were comfortably above the minimum legibility size for all observers, they were sized to scale on the computer to simulate a "real-world" viewing distance of $26.6 \mathrm{~m}(87.3 \mathrm{ft})$ at a viewing distance of $83 \mathrm{~cm}(32.7$ in) from the monitor in both the legibility and reaction time tasks. The screen height of each sign is included in appendix F.

\section{Glance Legibility}

The symbol signs were printed in their standard colors and presented on white background with $1 \mathrm{~ms}$ precision using a Gerbrands Model T-3B-1 three-field tachistoscope under the control of a G1159 Logic Interface. The fixation stimulus consisted of four short right angle segments that defined each comer of a square fixation field, $5 \mathrm{~cm}(1.97 \mathrm{in})(3.45$ ") on a side. After $2 \mathrm{~s}$, a sign appeared in the center of the area that the fixation square defined. White-area luminance for the fixation and sign stimuli was maintained at $77 \mathrm{~cd} / \mathrm{m}^{2}$ using a photocell in each channel connected to a digital multimeter.

\section{$\underline{\text { Reaction Time }}$}

The reaction time task was carried out using an Apple $330 \mathrm{~mm}$ (1.3 in) High-Resolution RGB monitor driven by a Raster Ops 364 24-bit video board under the control of a Macintosh 
IIci computer. Sign names were presented across the center of the monitor's screen in black upper case $\left[8 \mathrm{~mm}(0.32 \mathrm{in})=0.55^{\circ} \mathrm{high}\right]$ and lower case letters $\left(7 \mathrm{~mm}(0.28 \mathrm{in})\right.$ or $0.48^{\circ}$ high with descenders, $5.5 \mathrm{~mm}(0.22 \mathrm{in})$ or $0.38^{\circ}$ high without descenders $)$ on a white background. For each of the 18 signs in the test set, as well as the practice sign used to introduce participants to the task (Y INTERSECTION SYMBOL--MUTCD\#W2-5), a "foil" symbol sign alternative was selected from the MUTCD. ${ }^{(3)}$ Criteria used in selecting these foils were judged similarity of background color, symbol shape, and symbol size. Because the foils selected were standard signs, the degree of actual similarity between a test sign and its foil necessarily varied considerably. Test or foil signs were presented to scale and in their regulation color on a white background $\left(77 \mathrm{~cd} / \mathrm{m}^{2}\right)$ in the center area of the screen (see appendix $\left.\mathrm{G}\right)$. To maintain a constant viewing distance, participants viewed the screen from a chin rest. Participants carried out the reaction time task using three keys situated on a control console. If the sign that appeared on the trial matched the sign name that preceded it, the subject pressed the "SAME" key at the left-center position of the console; if the sign and sign name did not match (i.e., the foil sign had appeared), the subject pressed the "DIFFERENT" key at the right-centre position. To initiate the next trial, the observer pressed a "NEXT" key situated in the lower center of the console. Response accuracy and latency both were recorded by the computer.

\section{Procedure}

After completion of the refraction, acuity, and contrast sensitivity testing procedures, the glance legibility task was carried out, followed by reaction time. This order was utilized for two reasons: to avoid the effects of cueing on legibility thresholds that could occur from repeatedly seeing the signs during the reaction time task, and to enhance participants' familiarity with the signs prior to the reaction time task.

\section{$\underline{\text { Glance Legibility }}$}

After the task was explained to the participants, it was demonstrated using the practice sign. On the first trial, it was presented for $4 \mathrm{~ms}$ and then increased in $1 \mathrm{~ms}$ steps on each subsequent trial, until its exposure duration was sufficient to allow the participant to describe the sign to the same pre-determined feature scoring criterion that was used for each sign in the prior studies of day and night legibility (appendix H). The minimum exposure duration for correct identification was recorded as the threshold for each of the 18 signs. Participants were requested not to blink during a sign's presentation. Participants viewed the signs in one of six different random orders. In the young and middle-aged groups, one male and one female participant each received one of the orders. Among the older, where there were six additional participants, three of the orders were repeated among the men and the other three were repeated among the women. The scoring criteria for each sign was based on an identification of the critical details that distinguished it from other signs, and which were necessary to convey its message completely. Testing occurred in a single session in which participants proceeded at their own pace, taking rests as needed. They received an honorarium of $\$ 10.00 / \mathrm{h}$ for their time in the study.

\section{$\underline{\text { Reaction Time }}$}


IIci computer. Sign names were presented across the center of the monitor's screen in black upper case $\left[8 \mathrm{~mm}(0.32 \mathrm{in})=0.55^{\circ} \mathrm{high}\right]$ and lower case letters $\left(7 \mathrm{~mm}(0.28 \mathrm{in})\right.$ or $0.48^{\circ} \mathrm{high}$ with descenders, $5.5 \mathrm{~mm}$ ( $0.22 \mathrm{in})$ or $0.38^{\circ}$ high without descenders) on a white background. For each of the 18 signs in the test set, as well as the practice sign used to introduce participants to the task (Y INTERSECTION SYMBOL--MUTCD\#W2-5), a "foil" symbol sign alternative was selected from the MUTCD. ${ }^{(3)}$ Criteria used in selecting these foils were judged similarity of background color, symbol shape, and symbol size. Because the foils selected were standard signs, the degree of actual similarity between a test sign and its foil necessarily varied considerably. Test or foil signs were presented to scale and in their regulation color on a white background

$\left(77 \mathrm{~cd} / \mathrm{m}^{*}\right)$ in the center area of the screen (see appendix G). To maintain a constant viewing distance, participants viewed the screen from a chin rest. Participants carried out the reaction time task using three keys situated on a control console. If the sign that appeared on the trial matched the sign name that preceded it, the subject pressed the "SAME" key at the left-center position of the console; if the sign and sign name did not match (i.e., the foil sign had appeared), the subject pressed the "DIFFERENT" key at the right-centre position. To initiate the next trial, the observer pressed a "NEXT" key situated in the lower center of the console. Response accuracy and latency both were recorded by the computer.

\section{Procedure}

After completion of the refraction, acuity, and contrast sensitivity testing procedures, the glance legibility task was carried out, followed by reaction time. This order was utilized for two reasons: to avoid the effects of cueing on legibility thresholds that could occur from repeatedly seeing the signs during the reaction time task, and to enhance participants' familiarity with the signs prior to the reaction time task.

\section{$\underline{\text { Glance Legibility }}$}

After the task was explained to the participants, it was demonstrated using the practice sign. On the first trial, it was presented for $4 \mathrm{~ms}$ and then increased in $1 \mathrm{~ms}$ steps on each subsequent trial, until its exposure duration was sufficient to allow the participant to describe the sign to the same pre-determined feature scoring criterion that was used for each sign in the prior studies of day and night legibility (appendix H). The minimum exposure duration for correct identification was recorded as the threshold for each of the 18 signs. Participants were requested not to blink during a sign's presentation. Participants viewed the signs in one of six different random orders. In the young and middle-aged groups, one male and one female participant each received one of the orders. Among the older, where there were six additional participants, three of the orders were repeated among the men and the other three were repeated among the women. The scoring criteria for each sign was based on an identification of the critical details that distinguished it from other signs, and which were necessary to convey its message completely. Testing occurred in a single session in which participants proceeded at their own pace, taking rests as needed. They received an honorarium of $\$ 10.00 / \mathrm{h}$ for their time in the study.

\section{$\underline{\text { Reaction Time }}$}


After completion of the glance legibility task and a rest break, subjects were familiarized systematically with the practice sign and 18 test signs. To make the task easier for them, participants learned the names of signs in groups of four or five, following a two-stage process. In the first stage, by pressing the "NEXT" button for each step, the observer was shown the printed name of a sign on the monitor, then the sign itself, and finally, the sign's name and the sign together. The second stage was the same as the first, except that when the sign name appeared, the participant was asked to read it aloud and to visualize the image of the sign that had been named. As before, the next two button presses showed participants the sign and then the sign name and sign together, providing them with feedback as to the correctness of their visualized image. In this way, the participants proceeded through all 19 signs four or five at a time.

Upon completion of the initial familiarization procedure, participants received a practice trial using the "Y SYMBOL" practice sign. The words "Y SYMBOL" appeared on the screen and the participant was requested to visualize the appearance of the corresponding symbol sign. After $3 \mathrm{~s}$, a sign appeared which was either the Y SYMBOL sign, or a foil (SIDE ROAD 45 DEGREES). If the Y SYMBOL sign appeared, the participant was requested to press the SAME button as quickly as possible; if the SIDE ROAD sign appeared, the subject was to press the DIFFERENT button instead. When the response was incorrect, the sign did not disappear from the screen, and participants were instructed to hit the alternate key as quickly as possible. After 12 such practice trials (six SAME and six DIFFERENT), the same basic procedure was repeated for each sign in the test set, following the same random order received by that participant on the glance legibility task.

\section{RESULTS}

\section{Contrast Sensitivity}

Since no effects of gender were observed in an Age (3) x Gender (2) x Spatial Frequency (5) mixed design ANOVA on the log-transformed contrast sensitivity data, subsequent analyses were carried out collapsing across this variable. An Age x Spatial Frequency ANOVA indicated significant effects for age, $[\mathrm{F}(2,39)=9.86, \mathrm{p}<0.001]$, spatial frequency $[\mathrm{F}(4,156)=84.19$, $\mathrm{g}<0.001]$, and their interaction $[\mathrm{F}(8,156)=4.17, \mathrm{p}<0.001]$. The magnitude of the age difference increased at higher spatial frequencies. As can be seen in figure 10, the mean log contrast sensitivity functions (CSF's) of the age groups were either close to or within the population norms for the VCTS 6000 for observers aged 10 to 70 years. The young subjects' sensitivity exceeded the norms slightly at 3.0 and $6.0 \mathrm{c} / \mathrm{deg}$. Tukey HSD pair-wise comparisons (with Tukey-Kramer adjustment for unequal n's) confirmed a pattern of increasing age differences in contrast sensitivity as spatial frequency increased. There were no age differences at $1.5 \mathrm{c} / \mathrm{deg}$, but at 3.0 and $18.0 \mathrm{c} / \mathrm{deg}$ the sensitivity of the young exceeded that of the older group, and at 6.0 and $12.0 \mathrm{c} / \mathrm{deg}$ the sensitivity of the both the young and middle-aged exceeded that of the older. None of the sensitivity differences between the young and middle-aged drivers were significant.

To determine the utility of a single CSF-derived score for predicting glance legibility and reaction time, several composite measures were derived from individual log CSF's: peak sensitivity (the highest log sensitivity of each observer), peak spatial frequency (the spatial 
frequency at which the peak of the log contrast sensitivity function occurs), and high cutoff (the spatial frequency at which the log CSF is half of its peak amplitude). Because the CSF assessed by the VCTS did not fall below half of its peak value at low-spatial frequencies for several observers, it was not possible to derive low cutoff or bandwidth measures for them. As can be seen in table 25, Tukey pairwise comparisons revealed a significant decline with age in the peak sensitivity and high-cutoff measures, but not for peak spatial frequency.

Table 25. Age differences in derived contrast sensitivity measures.

\begin{tabular}{|c|c|c|c|c|}
\hline & Young & $\begin{array}{l}\text { Middle- } \\
\text { Aged }\end{array}$ & $\underline{\text { Older }}$ & $\underline{\text { Age Diff }}$ \\
\hline Log Peak Sensitivity & 2.36 & 2.29 & 2.18 & $\mathrm{ac}$ \\
\hline Peak Spatial Frequency (C/deg) 5. & 5.0 & 3.4 & - & \\
\hline High Cutoff $(\mathrm{C} / \mathrm{deg})$ & 10.9 & 10.5 & 7.5 & $\mathrm{ac}$ \\
\hline
\end{tabular}

Notes:

$\mathrm{a}=$ Significant difference $(\mathrm{p}<.02$ or better) between Young and Older Drivers

$\mathrm{c}=$ Significant difference $(\mathrm{P}<.02$ or better $)$ between Middle-Aged and Older Drivers

\section{Glance Legibility}

Glance legibility thresholds for each of the 18 signs in the test set are presented in table 26, including any age differences revealed by a series of one-way ANOVA's with followup TukeyKramer HSD pair-wise comparisons. Glance legibility thresholds for the three age groups sign categories are presented in table 27. Possible age and sex differences in the glance legibility of the three sign categories (Best, Intermediate and Worst) were analyzed in an Age (3) by Gender (2) by Sign Category (3) mixed-design ANOVA. Since no significant effects were revealed for gender, subsequent analyses were collapsed across that variable. An Age Group (3) by Sign Category (3) ANOVA found significant effects for both age $[\mathrm{F}(2,39)=9.37$, $\mathrm{p}<0.001]$, sign category $[\mathrm{F}(2,78)=72.40, \mathrm{p}<0.001]$, and the age by sign category interaction $[\mathrm{F}(4,78)=4.81$, $\mathrm{p}<0.011$. As can be observed in figure 11, differences between the age groups were least for signs in the Best category and greatest for those in the Worst one. Table 27 shows the pattern of age differences across the three sign categories.

\section{Reaction Time}

Mean reaction times to each of the 18 signs, including age differences, are presented in table 28. As for glance legibility, no gender differences in reaction time were revealed by an omnibus mixed design ANOVA, so gender was excluded from further analyses. An Age (3) by Sign Category (3) mixed design ANOVA showed significant effects for age $[\mathrm{F}(2,39)=11.71$, $\mathrm{p}<0.001]$ and sign category $[\mathrm{F}(2,78)=5.19, \mathrm{p}<0.011$, but no interaction between them $(\mathrm{p}=0.67)$. Increased driver age was associated with longer reaction times, and to a lesser extent, so too were changes in initial sign quality from Best to Worse (see figure 12 and table 29). 
Table 26. Mean glance legibility threshold (ms) and standard deviations (SD) of 18 symbol signs for young, middle-aged, and older drivers, ranked from overall lowest to highest.

\begin{tabular}{|c|c|c|c|c|c|c|}
\hline $\begin{array}{l}\text { Overall } \\
\text { Rank }\end{array}$ & $\begin{array}{l}\text { Sign Name } \\
\text { (MUTCD\#) }\end{array}$ & $\begin{array}{l}\text { Age } \\
\text { Diff }\end{array}$ & $\begin{array}{l}\text { Young } \\
(\mathrm{SD})\end{array}$ & $\begin{array}{c}\text { Middle- } \\
\text { Aged } \\
(\mathrm{SD})\end{array}$ & $\begin{array}{l}\text { Older } \\
(\mathrm{SD})\end{array}$ & $\begin{array}{c}\text { Overall } \\
\text { Means } \\
(\mathrm{SD})\end{array}$ \\
\hline 1 & $\begin{array}{l}\text { Hospital } \\
\text { (D9-2) }\end{array}$ & $\mathrm{a}$ & $\begin{array}{c}4.8 \\
(0.9)\end{array}$ & $\begin{array}{c}5.4 \\
(1.6)\end{array}$ & $\begin{array}{c}8.2 \\
(4.7)\end{array}$ & $\begin{array}{c}6.1 \\
(3.5)\end{array}$ \\
\hline 2. & $\begin{array}{l}\text { Phone } \\
\text { (D9-1) }\end{array}$ & $\mathrm{ac}$ & $\begin{array}{c}5.2 \\
(0.8)\end{array}$ & $\begin{array}{c}6.1 \\
(2.0)\end{array}$ & $\begin{array}{c}9.7 \\
(5.3)\end{array}$ & $\begin{array}{c}7.0 \\
(4.1)\end{array}$ \\
\hline 3. & $\begin{array}{l}\text { Cross Road } \\
\text { (W2-1) }\end{array}$ & $\mathrm{a}$ & $\begin{array}{c}6.8 \\
(1.9)\end{array}$ & $\begin{array}{c}8.2 \\
(2.1)\end{array}$ & $\begin{array}{l}10.4 \\
(3.5)\end{array}$ & $\begin{array}{c}8.4 \\
(3.1)\end{array}$ \\
\hline 4. & $\begin{array}{l}\text { Keep Right } \\
(\mathrm{R} 4-7)\end{array}$ & $\mathrm{a}$ & $\begin{array}{c}5.5 \\
(1.5)\end{array}$ & $\begin{array}{c}7.4 \\
(2.5)\end{array}$ & $\begin{array}{c}13.9 \\
(12.6)\end{array}$ & $\begin{array}{r}9.0 \\
(9.1)\end{array}$ \\
\hline 5. & $\begin{array}{l}\text { Right Curve } \\
\text { (W1-2R) }\end{array}$ & $\mathrm{ac}$ & $\begin{array}{c}7.2 \\
(1.2)\end{array}$ & $\begin{array}{c}8.8 \\
(2.80)\end{array}$ & $\begin{array}{l}12.9 \\
(5.7)\end{array}$ & $\begin{array}{c}9.6 \\
(4.7)\end{array}$ \\
\hline 6. & $\begin{array}{l}\text { No Trucks } \\
\text { (R5-2) }\end{array}$ & $\mathrm{a}$ & $\begin{array}{c}5.7 \\
(1.2)\end{array}$ & $\begin{array}{c}8.2 \\
(4.0)\end{array}$ & $\begin{array}{c}16.3 \\
(13.2)\end{array}$ & $\begin{array}{c}10.0 \\
(10.0)\end{array}$ \\
\hline 7. & $\begin{array}{l}\text { Bicycle Crossing } \\
\text { (W11-1) }\end{array}$ & $\mathrm{a}$ & $\begin{array}{c}6.7 \\
(2.5)\end{array}$ & $\begin{array}{c}9.7 \\
(5.6)\end{array}$ & $\begin{array}{l}14.9 \\
(9.3)\end{array}$ & $\begin{array}{l}10.4 \\
(7.7)\end{array}$ \\
\hline 8. & $\begin{array}{l}\text { No Parking } \\
\text { (RS-3a) }\end{array}$ & $\mathrm{a}$ & $\begin{array}{c}6.7 \\
(3.3)\end{array}$ & $\begin{array}{l}10.0 \\
(5.8)\end{array}$ & $\begin{array}{l}15.7 \\
(8.2)\end{array}$ & $\begin{array}{l}10.8 \\
(7.4)\end{array}$ \\
\hline 9. & $\begin{array}{l}\text { Divided Highway Ends } \\
\text { (W6-2) }\end{array}$ & $\mathrm{a}$ & $\begin{array}{c}8.3 \\
(2.5)\end{array}$ & $\begin{array}{l}12.8 \\
(7.5)\end{array}$ & $\begin{array}{c}18.1 \\
(10.3)\end{array}$ & $\begin{array}{l}13.1 \\
(8.8)\end{array}$ \\
\hline 10. & $\begin{array}{l}\text { Nat Net Route Prohibited } \\
\text { (R14-5) }\end{array}$ & $\mathrm{a}$ & $\begin{array}{c}7.6 \\
(2.8)\end{array}$ & $\begin{array}{c}14.3 \\
(11.3)\end{array}$ & $\begin{array}{c}23.1 \\
(17.7)\end{array}$ & $\begin{array}{c}15.0 \\
(14.5)\end{array}$ \\
\hline 11. & $\begin{array}{l}\text { No U-Turn } \\
\text { (R3-4) }\end{array}$ & $\mathrm{a}$ & $\begin{array}{l}9.17 \\
(4.6)\end{array}$ & $\begin{array}{l}13.7 \\
(9.2)\end{array}$ & $\begin{array}{c}25.2 \\
(19.3)\end{array}$ & $\begin{array}{c}16.0 \\
(15.3)\end{array}$ \\
\hline 12. & $\begin{array}{l}\text { Advance Flagger } \\
\text { (W20-7a) }\end{array}$ & $\mathrm{a}$ & $\begin{array}{l}11.8 \\
(2.5)\end{array}$ & $\begin{array}{r}15.4 \\
(6.5)\end{array}$ & $\begin{array}{l}21.4 \\
(9.0)\end{array}$ & $\begin{array}{l}16.2 \\
(8.0)\end{array}$ \\
\hline
\end{tabular}

Notes:

$\mathrm{a}=$ Significant difference $(\mathrm{p}<.05$ or better $)$ between Young and Older Drivers

$\mathrm{c}=$ Significant difference $(\mathrm{p}<.05$ or better) between Middle-Aged and Older Drivers 
Table 26. Mean glance legibility threshold (ms) and standard deviations (SD) of 18 symbol signs for young, middle-aged, and older drivers, ranked from overall lowest to highest (continued).

\begin{tabular}{|c|c|c|c|c|c|c|}
\hline $\begin{array}{l}\text { Overall } \\
\text { Rank }\end{array}$ & $\begin{array}{l}\text { Sign Name } \\
\text { (MUTCD\#) }\end{array}$ & $\begin{array}{l}\text { Age } \\
\text { Diff }\end{array}$ & $\begin{array}{l}\text { Young } \\
\text { (SD) }\end{array}$ & $\begin{array}{l}\text { Middle } \\
\text { Aged } \\
\text { (SD) }\end{array}$ & $\begin{array}{l}\text { Older } \\
\text { (SD) }\end{array}$ & $\begin{array}{c}\text { Overall } \\
\text { Means } \\
(\text { SD) }\end{array}$ \\
\hline$\overline{13}$ & $\begin{array}{l}\text { Campfire } \\
\text { (RA-030) }\end{array}$ & $\bar{a}$ & $\begin{array}{l}\frac{(N D)}{9.8} \\
(6.7)\end{array}$ & $\begin{array}{l}\frac{(N D)}{13.1} \\
(11.5)\end{array}$ & $\begin{array}{l}\frac{(\mathrm{DN})}{27.0} \\
(22.4)\end{array}$ & $\begin{array}{l}\frac{(N D)}{16.6} \\
(17.8)\end{array}$ \\
\hline 14. & $\begin{array}{l}\text { RV Sanitary Station } \\
\text { (D9-12) }\end{array}$ & $\mathrm{a}$ & $\begin{array}{c}14.2 \\
(18.6)\end{array}$ & $\begin{array}{c}17.3 \\
(11.5)\end{array}$ & $\begin{array}{c}30.4 \\
(19.6)\end{array}$ & $\begin{array}{c}20.6 \\
(18.6)\end{array}$ \\
\hline 15 . & $\begin{array}{l}\text { Mandatory Seatbelt } \\
\text { (R16-1) }\end{array}$ & $\mathrm{a}$ & $\begin{array}{l}12.9 \\
(7.9)\end{array}$ & $\begin{array}{c}22.4 \\
(18.2)\end{array}$ & $\begin{array}{c}28.3 \\
(16.9)\end{array}$ & $\begin{array}{c}21.2 \\
(16.3)\end{array}$ \\
\hline 16. & $\begin{array}{l}\text { Skating } \\
(\mathrm{RS}-010)\end{array}$ & $\mathrm{a}$ & $\begin{array}{c}15.8 \\
(15.4)\end{array}$ & $\begin{array}{c}20.7 \\
(14.6)\end{array}$ & $\begin{array}{l}32.9 \\
(18.5)\end{array}$ & $\begin{array}{c}23.2 \\
(17.9)\end{array}$ \\
\hline 17. & $\begin{array}{l}\text { Ranger Station } \\
(\mathrm{RG}-170)\end{array}$ & $\mathrm{a}$ & $\begin{array}{l}17.6 \\
(14.7)\end{array}$ & $\begin{array}{c}26.3 \\
(17.8)\end{array}$ & $\begin{array}{c}35.4 \\
(14.4)\end{array}$ & $\begin{array}{l}26.4 \\
(16.9)\end{array}$ \\
\hline 18. & $\begin{array}{l}\text { Pavement Ends } \\
\text { (W8-3a) }\end{array}$ & ac & $\begin{array}{c}15.7 \\
(10.3)\end{array}$ & $\begin{array}{c}26.3 \\
(12.5)\end{array}$ & $\begin{array}{c}40.7 \\
(17.0)\end{array}$ & $\begin{array}{c}27.6 \\
(17.9)\end{array}$ \\
\hline
\end{tabular}

Table 27. Mean glance legibility threshold (ms) for best, intermediate, and worst signs among young, middle-aged, and older drivers.

\begin{tabular}{|c|c|c|c|c|c|}
\hline & & Middle- & & $\underline{\text { Overall }}$ & \\
\hline$\underline{\operatorname{Sign}}$ & Young & Aged & Older & $\overline{\text { Means }}$ & Age Diff \\
\hline Best & 6.4 & 8.3 & 13.4 & 9.4 & $\mathrm{ac}$ \\
\hline Intermediate & 9.4 & 12.8 & 21.5 & 14.6 & $\mathrm{ac}$ \\
\hline Worst & 12.7 & 19.9 & 29.2 & 20.6 & $\mathrm{a}$ \\
\hline
\end{tabular}

Notes:

$\mathrm{a}=$ Significant difference $(\mathrm{p}<.05$ or better $)$ between Young and Older Drivers.

$\mathrm{c}=$ Significant difference $(\mathrm{P}<.05$ or better $)$ between Middle-Aged and Older Drivers. 


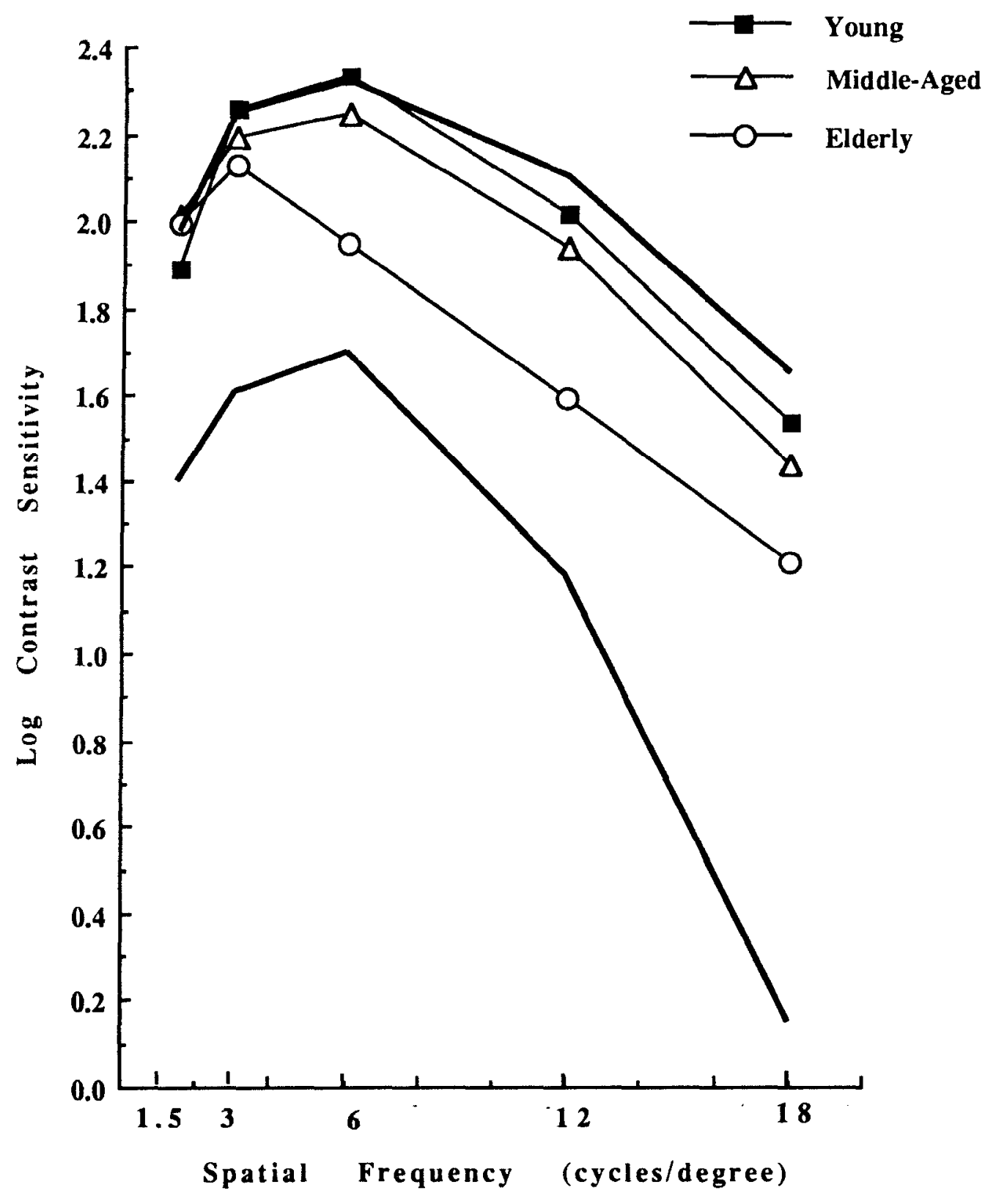

Figure 10. Log contrast sensitivity functions for young, middle-aged, and elderly drivers with VCTS norms (bold lines). 
Table 28. Mean reaction time and standard deviations (ms) for 18 symbol signs for young, middle-aged, and older drivers, ranked from fastest to slowest.

\begin{tabular}{|c|c|c|c|c|c|c|}
\hline $\begin{array}{l}\text { Overall } \\
\underline{\text { Rank }}\end{array}$ & $\begin{array}{l}\text { Sign Name } \\
\text { (MUTCD\#) }\end{array}$ & $\begin{array}{l}\text { Age } \\
\text { Diff }\end{array}$ & $\begin{array}{l}\text { Young } \\
\underline{(\mathrm{SD})}\end{array}$ & $\begin{array}{l}\text { Middle- } \\
\text { Aged } \\
\underline{(\mathrm{SD})}\end{array}$ & $\begin{array}{l}\text { Older } \\
\underline{(\mathrm{SD})}\end{array}$ & $\begin{array}{l}\text { Overall } \\
\text { Means } \\
(\mathrm{SD})\end{array}$ \\
\hline 1 & $\begin{array}{l}\text { Phone } \\
\text { (D9-1) }\end{array}$ & $\mathrm{a}$ & $\begin{array}{c}516.1 \\
(94.0)\end{array}$ & $\begin{array}{r}642.4 \\
(167.2)\end{array}$ & $\begin{array}{r}683.3 \\
(158.5)\end{array}$ & $\begin{array}{r}633.9 \\
(159.2)\end{array}$ \\
\hline 2. & $\begin{array}{l}\text { Hospital } \\
\text { (D9-2) }\end{array}$ & $\mathrm{ac}$ & $\begin{array}{r}471.6 \\
(114.5)\end{array}$ & $\begin{array}{r}606.9 \\
(169.0)\end{array}$ & $\begin{array}{r}781.6 \\
(229.4)\end{array}$ & $\begin{array}{r}640.0 \\
(224.5)\end{array}$ \\
\hline 3. & $\begin{array}{l}\text { Bicycle Crossing } \\
\text { (W11-1) }\end{array}$ & $\mathrm{ac}$ & $\begin{array}{l}466.2 \\
(73.9)\end{array}$ & $\begin{array}{r}589.7 \\
(136.6)\end{array}$ & $\begin{array}{r}842.1 \\
(274.0)\end{array}$ & $\begin{array}{r}652.6 \\
(254.2)\end{array}$ \\
\hline 4. & $\begin{array}{l}\text { Campfire } \\
\text { (RA-030) }\end{array}$ & $\mathrm{a}$ & $\begin{array}{r}580.8 \\
(205.0)\end{array}$ & $\begin{array}{r}596.5 \\
(135.1)\end{array}$ & $\begin{array}{r}776.3 \\
(255.8)\end{array}$ & $\begin{array}{r}671.2 \\
(228.4)\end{array}$ \\
\hline 5. & $\begin{array}{l}\text { Advance Flagger } \\
(\mathrm{W} 20-7 \mathrm{a})\end{array}$ & $\mathrm{a}$ & $\begin{array}{l}522.2 \\
(79.5)\end{array}$ & $\begin{array}{r}661.1 \\
(126.8)\end{array}$ & $\begin{array}{r}786.7 \\
(233.3)\end{array}$ & $\begin{array}{r}676.6 \\
(202.4)\end{array}$ \\
\hline 6. & $\begin{array}{l}\text { Mandatory Seatbelt } \\
\text { (R16-1) }\end{array}$ & $\mathrm{a}$ & $\begin{array}{r}490.6 \\
(109.4)\end{array}$ & $\begin{array}{r}710.8 \\
(302.6)\end{array}$ & $\begin{array}{r}803.1 \\
(336.5)\end{array}$ & $\begin{array}{r}688.1 \\
(303.5)\end{array}$ \\
\hline 7. & $\begin{array}{l}\text { No Parking } \\
\text { (R8-3a) }\end{array}$ & $\mathrm{ac}$ & $\begin{array}{r}505.8 \\
(116.6)\end{array}$ & $\begin{array}{r}672.4 \\
(151.4)\end{array}$ & $\begin{array}{r}883.3 \\
(310.2)\end{array}$ & $\begin{array}{r}707.2 \\
(274.7)\end{array}$ \\
\hline 8. & $\begin{array}{l}\text { Pavement Ends } \\
\text { (W8-3a) }\end{array}$ & $\mathrm{ac}$ & $\begin{array}{r}515.9 \\
(142.0)\end{array}$ & $\begin{array}{r}638.0 \\
(126.2)\end{array}$ & $\begin{array}{r}916.7 \\
(366.8)\end{array}$ & $\begin{array}{r}710.2 \\
(310.8)\end{array}$ \\
\hline 9. & $\begin{array}{l}\text { RV Sanitary Station } \\
\text { (D9-12) }\end{array}$ & $\mathrm{a}$ & $\begin{array}{r}584.7 \\
(152.3)\end{array}$ & $\begin{array}{r}675.1 \\
(103.1)\end{array}$ & $\begin{array}{r}836.0 \\
(293.2)\end{array}$ & $\begin{array}{r}718.6 \\
(237.9)\end{array}$ \\
\hline 10. & $\begin{array}{l}\text { No U-Turn } \\
\text { (R3-4) }\end{array}$ & $\mathrm{a}$ & $\begin{array}{r}558.6 \\
(166.1)\end{array}$ & $\begin{array}{r}728.4 \\
(237.1)\end{array}$ & $\begin{array}{r}883.5 \\
(278.6)\end{array}$ & $\begin{array}{r}743.5 \\
(270.8)\end{array}$ \\
\hline 11. & $\begin{array}{l}\text { Ranger Station } \\
(\mathrm{RG}-170)\end{array}$ & $\mathrm{a}$ & $\begin{array}{r}571.4 \\
(174.2)\end{array}$ & $\begin{array}{r}711.6 \\
(150.9)\end{array}$ & $\begin{array}{r}892.8 \\
(412.7)\end{array}$ & $\begin{array}{r}745.3 \\
(321.9)\end{array}$ \\
\hline 12. & $\begin{array}{l}\text { Cross Road } \\
\text { (W2-1) }\end{array}$ & $\mathrm{ac}$ & $\begin{array}{l}498.7 \\
(99.0)\end{array}$ & $\begin{array}{r}689.1 \\
(160.5)\end{array}$ & $\begin{array}{r}989.4 \\
(365.7)\end{array}$ & $\begin{array}{r}745.7 \\
(330.9)\end{array}$ \\
\hline
\end{tabular}

Notes:

$\mathrm{a}=$ Significant difference $(\mathrm{p}<.05$ or better) between Young and Older Drivers.

$\mathrm{c}=$ Significant difference $(\mathrm{p}<.05$ or better) between Middle-Aged and Older Drivers. 
Table 28. Mean reaction time and standard deviations (ms) for 18 symbol signs for young, middle-aged, and older drivers, ranked from fastest to slowest (continued).

\begin{tabular}{|c|c|c|c|c|c|c|}
\hline Overall & Sign Name & Age & Young & $\begin{array}{l}\text { Middle } \\
\text { Aged }\end{array}$ & Older & $\begin{array}{l}\text { Overall } \\
\text { Means }\end{array}$ \\
\hline$\underline{\text { Rank }}$ & $\underline{\text { (MUTCD\#) }}$ & $\underline{\text { Diff }}$ & $(\mathrm{SD})$ & $(\mathrm{SD})$ & (SD) & $(\mathrm{SD})$ \\
\hline 13. & $\begin{array}{l}\text { Skating } \\
(\mathrm{RS}-010)\end{array}$ & $\mathrm{a}$ & $\begin{array}{r}584.9 \\
(188.0)\end{array}$ & $\begin{array}{r}705.2 \\
(176.4)\end{array}$ & $\begin{array}{r}909.4 \\
(340.7)\end{array}$ & $\begin{array}{r}753.2 \\
(292.6)\end{array}$ \\
\hline 14. & $\begin{array}{l}\text { Nat Net Route Prohibited } \\
(\mathrm{R} 14-5)\end{array}$ & ac & $\begin{array}{l}526.7 \\
(95.4)\end{array}$ & $\begin{array}{r}684.3 \\
(106.3)\end{array}$ & $\begin{array}{r}1084.7 \\
(491.1)\end{array}$ & $\begin{array}{r}785.2 \\
(408.3)\end{array}$ \\
\hline 15. & $\begin{array}{l}\text { No Trucks } \\
\text { (R5-2) }\end{array}$ & $\mathrm{a}$ & $\begin{array}{r}561.2 \\
(151.5)\end{array}$ & $\begin{array}{r}796.1 \\
(257.3)\end{array}$ & $\begin{array}{r}1045.4 \\
(357.9)\end{array}$ & $\begin{array}{r}820.9 \\
(344.8)\end{array}$ \\
\hline 16. & $\begin{array}{l}\text { Divided Highway Ends } \\
\text { (W6-2) }\end{array}$ & $\mathrm{a}$ & $\begin{array}{r}635.4 \\
(197.1)\end{array}$ & $\begin{array}{r}896.3 \\
(358.0)\end{array}$ & $\begin{array}{r}1139.2 \\
(482.4)\end{array}$ & 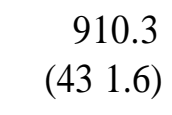 \\
\hline 17. & $\begin{array}{l}\text { Right Curve } \\
\text { (W1-2R) }\end{array}$ & $\mathrm{a}$ & $\begin{array}{r}684.0 \\
(281.6)\end{array}$ & $\begin{array}{r}836.4 \\
(335.5)\end{array}$ & $\begin{array}{r}1191.7 \\
(595.0)\end{array}$ & $\begin{array}{r}924.1 \\
(498.4)\end{array}$ \\
\hline 18. & $\begin{array}{l}\text { Keep Right } \\
(\mathrm{R} 4-7)\end{array}$ & $\mathrm{a}$ & $\begin{array}{r}645.5 \\
(231.3)\end{array}$ & $\begin{array}{r}982.3 \\
(437.0)\end{array}$ & $\begin{array}{r}1298.8 \\
(871.8)\end{array}$ & $\begin{array}{r}995.5 \\
(675.5)\end{array}$ \\
\hline
\end{tabular}

Table 29. Mean reaction time threshold (ms) for best, intermediate, and worst signs among young, middle-aged, and older drivers.

\begin{tabular}{|c|c|c|c|c|c|}
\hline & & Middle- & & Overall & \\
\hline Sign & Young & Aged & $\underline{\text { Older }}$ & $\overline{\text { Means }}$ & Age Diff \\
\hline Best & 542.4 & 727.6 & 951.4 & 740.5 & $\mathrm{a}$ \\
\hline Intermediate & 545.0 & 696.2 & 891.2 & 710.8 & $\mathrm{ac}$ \\
\hline Worst & 505.9 & 653.3 & 888.1 & 761.7 & $\mathrm{ac}$ \\
\hline
\end{tabular}

Notes:

$\mathrm{a}=$ Significant difference ( $\mathrm{p}<.05$ or better) between Young and Older Drivers

$\mathrm{c}=$ Significant difference $(\mathrm{p}<.05$ or better) between Middle-Aged and Older Drivers 


\section{Relationships Between Visual Measures And Glance Legibility Or Reaction Time}

The relationships between the visual measures of best acuity, peak sensitivity, high cutoff, and peak spatial frequency and glance legibility, or reaction time were determined with Pearson product-moment correlations across the age groups, and also separately within each age group. Acuity and glance legibility threshold were strongly related over the three age groups $(r=0.74$, $\mathrm{p}<0.001)$, as well as within each age group: young $(\underline{r}=0.81, p<0.001)$, middle-aged $(\underline{r}=0.64$, $\mathrm{p}<0.05)$, and older $(\mathrm{r}=0.61, \mathrm{p}<0.01)$. Transformation of these correlations to Fisher's $\mathrm{Z}$ scores, however, revealed no significant differences between the age groups in the strength of the acuity/legibility relationship. The three contrast sensitivity measures also correlated with legibility scores when assessed across age. For high cutoff spatial frequency, $\underline{r}=0.58, p<01$, for peak sensitivity, $\underline{r}=0.41, \mathrm{p}<0.01$, and peak spatial frequency, $\underline{r}=0.45, \mathrm{p}<0.01$. Within each age group, however, the high-cutoff spatial frequency was related to glance legibility only among middle-aged drivers $(r=0.64, \mathrm{p}<0.05)$.

Although acuity correlated quite highly with reaction time overall $(r=0.66, p<0.001)$, the relationship was not significant within any age group. A similar pattern of results was seen for the contrast sensitivity measures. High cutoff $(\underline{r}=0.34, p<0.05)$, peak sensitivity $(\underline{r}=0.37, p<$ $0.05)$, and peak spatial frequency $(\underline{r}=0.39, p<0.01)$ were all correlated with overall reaction time, but none of the corresponding correlations were significant within individual age groups.

Relationships Between Glance Legibility, Reaction Time, And Other Measures Of Sign Effectiveness

To determine the degree of dependence between the two measures, Pearson correlations were determined between glance legibility and reaction time, both overall and separately for each age group. When assessed across the three age groups, the two measures showed a significant degree of association $(\underline{r}=0.66, p<0.01)$. Within age groups, reaction time and legibility were significantly related only among older drivers $(\underline{r}=0.51, p<0.05)$.

To evaluate the relationship between the various measures that have been used in evaluating the 18 signs in the test set, Spearman rank correlations were calculated between the findings of the present study and those from earlier studies in this series: on Day Legibility, Comprehension, and Night Legibility. Glance legibility was very strongly related to both day $\left(r_{s}=0.83, p<0.01\right)$ and night legibility $\left(r_{s}=0.79, p<0.01\right)$, but less robustly to comprehension $\left(r_{s}=0.65, p<0.01\right)$. Day and night legibility were almost perfectly related $(r=0.97, p<0.001)$. Reaction time, however, was not significantly correlated with any of the other measures of sign effectiveness.

\section{DISCUSSION}

\section{Glance Legibility}

Very marked differences in glance legibility exist between signs that were evaluated. As can be seen in table 26, the mean minimum exposure duration required for legibility of the best sign (HOSPITAL) was less than a quarter that of the worst (PAVEMENT ENDS). Generally, glance legibility appeared to be poorest for figurally complex or detailed signs. Although no differences were seen between men and women drivers, age differences were seen on every sign tested (primarily between young and older drivers). Although the thresholds of middle-aged drivers were generally a little higher from those of the young, they were more similar to them 
than the older drivers (e.g., see figure 11). The glance legibility thresholds of older drivers were more affected by the sign category (i.e., Best, Intermediate, Worst) than were younger drivers. This pattern of increasing age differences in going from "Best" to "Worst" signs can be seen quite clearly in figure 12 . One of the implications of this finding is that the glance legibility of signs can be enhanced for drivers of all ages, especially older ones, by optimizing their legibility distance and ease of comprehension.

\section{Reaction Time}

These marked differences between signs in the latency with which drivers were able to determine the correspondence between a sign and a sign name that had preceded it. Unlike for glance legibility, however, there did not appear to be a consistent relationship between the figural complexity of signs and the latency with which drivers responded to them. Some of the signs that were responded to most quickly were figurally simple ones (e.g., PHONE, HOSPITAL), but so too were some of the signs that elicited the longest reaction times (e.g., RIGHT CURVE). Conversely, some of the signs that generated the shortest reaction times were figurally complex (e.g., BICYCLE CROSSING, CAMPFIRE). This finding suggests that contextual variables may be more important determinants of reaction time of the type measured here than are the characteristics of individual signs. Such contextual variables include familiarity of the sign, similarity between the test sign and the foil from which it had to be distinguished, and the degree of similarity between a given sign and others in the test set. For example, the two best tested signs, PHONE and HOSPITAL, are very common symbols, quite different from their foils, and unlike any of the other signs in the test set. In contrast, the two signs producing the longest mean reaction time (RIGHT CURVE and KEEP RIGHT) are less familiar, similar to one another figurally and semantically, and relatively difficult to distinguish from the foil signs with which they were paired (RIGHT REVERSE CURVE, STRAIGHT or LEFT) respectively.

Older drivers were significantly slower than young drivers on every sign tested, and significantly slower than middle-aged drivers on a third of the signs (see table 29). Although middle-aged drivers were also slower than young ones on all signs, the difference was not significant for any sign. Also, unlike glance legibility, the magnitude of the age differences showed little variation from the "Best" to the "Worst" sign category. Since participants had been familiarized with all the signs before the reaction time task began, and the signs were well above legibility threshold in size, the small but significant decrease in reaction time from the Best to Worst categories was nonetheless surprising and without obvious explanation. Four of the six Best signs were, however, ranked in the bottom half of the test set on reaction time, including the KEEP RIGHT and RIGHT CURVE signs that as already noted, produced the worst and secondworst mean reaction times. Thus, the same kind of factors cited above to explain the lack of relationship between figural complexity and reaction time: familiarity, test-set inter-sign similarity, test sign-foil similarity, in combination with a large sample size for evaluation of the sign category effect $(\mathrm{N}=42)$, may have contributed to the sign category effect that was observed. 


\section{Visual Functioning, Glance Legibility, And Reaction Time}

Consistent with prior studies, the acuity of older participants was significantly worse than that of their younger counterparts. ${ }^{(171)}$ The pattern of increasing age differences in contrast sensitivity at higher spatial frequencies was also in keeping with previous studies. ${ }^{(114,125)}$

When acuity and composite measures of contrast sensitivity were compared as predictors of glance legibility, acuity proved to be the more useful of the two measures for all three age groups. Of the three derived contrast sensitivity measures, only high cutoff was associated with glance legibility, and only among middle-aged drivers. Peak sensitivity and peak spatial frequency were not significantly related to glance legibility for any age group. The predictive utility of acuity may reflect the fine-detailed criterion used in determining legibility thresholds. Certainly, the requirement that observers report all of a sign's critical features no matter how small increased the acuity demands of the task.

The relationships between reaction time and all visual measures were weak, likely reflecting the fact that the reaction time task did not make strong demands on spatial vision. The correlation between acuity and mean reaction time across age groups probably reflected only their common age decline since the relationship was not seen within any single age group. The contrast sensitivity measures were not significantly related to reaction time performance within or across age groups.

\section{Relationships Among Measures Of Sign Effectiveness}

Although signs can be assessed on a variety of different measures, little research has examined the extent to which each measure provides unique formation about their effectiveness. Roberts, Lareau, and Welch compared signs on five different measures (time to indicate a sign's meaning, comprehension, response certainty, preference, and minimum exposure time). ${ }^{(147)}$ They found a significant correlation only between time taken to indicate a sign's meaning and certainty of response, suggesting that most of their five measures assessed different aspects of symbol sign effectiveness.

In the present study, glance legibility and reaction time appeared to provide overlapping information about symbol signs only when they were assessed across all three age groups. However, when the same relationship was assessed within age groups to avoid the age decline common to both measures, a significant relationship was seen only for older drivers. Presumably, this is attributable to the greater variability that is characteristic of older persons (e.g., see tables 25 and 27), and to the greater number of older drivers tested. The problem of spurious correlations between the measures due to an age decline common to them all was avoided by determining the rank-order correlations between glance legibility, reaction time, comprehension, day legibility, and night legibility for the same M-sign test set. By this measure, the glance legibility of symbol signs was highly related to their legibility in both day and night conditions ( $\mathrm{r}$ about .80), and to a lesser but important degree, to the level at which they were comprehended $(r=.65)$. This suggests that if a sign is designed to be effective on one of these three dimensions, it is likely to be effective on the other two as well. This is particularly true of the relationship between day and night legibility, which was so strong that it suggests that legibility distance is a singular property of signs. The speed with which drivers can determine the presence or absence of a sign versus a similar alternative, however, was not related to any of 
the other criterion measures. To the extent that response latency. for discriminating similar signs is critical to their operational effectiveness, it may be necessary to design and evaluate it separately from most other measures. 
121 


\section{CHAPTER 8: CONSPICUITY OF SYMBOL SIGNS}

Highway signs can be effective only to the degree that they are noticed in time for its message to guide driver behavior appropriately. If a sign captures the driver's attention in the absence of a deliberate search, the property by which it does so is referred to as "attention conspicuity"; if a sign is detected as a result of a deliberate search by the driver, the task involves "search conspicuity."(28) Conspicuity of both types depends on a complex interplay of a wide variety of factors that include both characteristics of the sign, such as its size, color, brightness, contrast, familiarity, and location in relation to the drivers line of sight, and characteristics of the context in which it is located, such as its color(s), texture, illumination, and the presence of competing stimuli in the vicinity. The goals of this study were to compare a test set of symbol highway signs on search conspicuity among drivers of different age, and to evaluate the relationship between search conspicuity, other measures of sign effectiveness, and spatial vision.

Search conspicuity for a test set of 18 signs was compared among young, middle-aged, and older drivers. Given the wide range of differences in the signs in the test set in regard to color, size, and similarity to other signs, marked differences between them were anticipated. Also, given the age-related decline that is typically observed on visual search tasks. ${ }^{(147,149,174)}$ It was expected that older drivers would take longer than middle-aged or younger ones to carry out the conspicuity search task.

\section{METHOD}

\section{Subjects}

Three age groups of licensed, community-resident drivers participated in the study: a young group ( 6 men and 6 women), $\underline{\mathrm{M}}$ age $=26.7$ years (range 21 to 34), a middle-aged group ( 6 men and 6 women), $\underline{\mathrm{M}}$ age $=49.83$ (range 42 to 59), and an older group ( 9 men and 9 women), $\underline{\mathrm{M}}$ age $=67.9$ (range 60 to 81 ). A larger sample of older drivers was tested due to the age-related increase in inter- and intra-individual variability. The mean number of years of education was 15.9 years (range 12 to 20) for the young, 14.1 years (range 12 to 17) for the middle-aged, and 14.3 years (range 8 to 22) for the older. None of the possible age differences in education were significant ( $\mathrm{p}=0.18$ ), (see table 30 .) Overall, the participants were in good general health, although older drivers ( 5 of 18 ) were more likely than either young ( 0 of 12$)$ or middle-aged ( 1 of 12) ones to report a chronic illness or complaint. Older drivers (6 of 18) were also more likely than the young ( 0 of 12 ) or middle-aged ( 1 of 12) ones to take one or more medications regularly. The visual health of the younger participants was generally good; no young, and only one middle-aged participant reported having chronic visual problems. Eight of the older participants, however, reported one or more chronic visual problems, most of them age-related (3 cataracts, 1 night blindness, 1 macular degeneration, 1 color blindness, 1 ulcerated eye, and 1 burst blood vessels). Mean binocular photopic Landolt C acuity levels, assessed at the test distance of $83 \mathrm{~cm}$ (32.7 in), were: 0.71 minarc (range .70 to .75 ) for the young, 0.74 minarc (range .70 to .93 ) for the middle-aged and 1.07 minarc (range .70 to 1.63$)$ for the older participants, respectively [( 1.0 minarc $=6 / 6(20 / 20)]$. The acuity levels of the older were lower than those of both the young $(p$ $<.01)$ and the middle-aged drivers ( $\mathrm{p}<.01$ ), which were not significantly different from one another $(\mathrm{p}=.92)$. Information regarding the driving experience of the participants is provided in table 30 . 
Table 30. Participant demographics: conspicuity. Average number of years driving experience as a function of age and sex

$\begin{array}{lccc}\text { Sex } & \text { Young } & \frac{\text { Age Group }}{\text { Middle-Aged }} & \text { Older } \\ \text { Males } & 8.2 & 29.8 & 51.6 \\ \text { Females } & 12.2 & 30.7 & 40.7 \\ \text { Total } & 10.2 & 30.3 & 46.1\end{array}$

Average number of years held a driver's license

$\begin{array}{lll}\text { Young } & \frac{\text { Age Group }}{\text { Middle-Aged }} & \frac{\text { Older }}{44.7}\end{array}$

Percentage of drivers by distance driven last 12 months as a function $\underline{\mathrm{o}} \mathrm{f}$

$\begin{array}{lccc}\frac{\text { Distance Driven }}{\text { (miles) }} & \text { Young } & \frac{\text { Age Group }}{\text { Middle-Aged }} & \text { Older } \\ \text { under } 2,000 & 33.3 & 25.0 & 16.7 \\ 2,000-4,999 & 33.3 & 25.0 & 44.4 \\ 5,000-9,999 & 16.7 & 33.3 & 16.7 \\ 10,000-14,999 & 8.3 & & 22.2 \\ 15,000-19,999 & 8.3 & 16.7 & \\ 20,000 \text { or more } & & & \end{array}$

Percentage of drivers by driving environment as a function of age

Driving Environment

Rural/sparsely populated

Small town

Suburban

High-density urban

Highway
Age Group

Young $\quad \underline{\text { Middle-Aged }}$

8.3

41.7

50.0 $\underline{\text { Older }}$

11.1

38.9

38.9

11.1

Percentape of drivers by night driving as a function of age

Percentage

Night Driving

$0-5$

$6-10$

11- 15

16-20

21-30

$31-40$

41-50

51 or more
Age Group

Young

8.3

16.7

8.3

16.7

16.7

33.3
Middle-Aged

16.7

16.7

8.3

25.0

16.7

8.3

8.3 $\underline{\text { Older }}$

22.2

16.7

22.2

22.2

5.6

5.6

5.6 
Table 30. Participant demographics: conspicuity (continued).

Percentage of drivers by driving done during rush hour as a function of age

\section{$\underline{\text { Percentage }}$}

$\underline{\text { Rush Hour }}$

$0-5$

$6-10$

$11-15$

16-20

21-30

$31-40$

$41-50$

51 or more
Age Group

$\underline{\text { Middle-Aged }}$

25.0

8.3

22.2

33.3

11.1

$16.7 \quad 27.8$

$25.0 \quad 5.6$

16.7

8.3

Percentage of drivers by driving done on the highway as a function of age

\section{Percentage}

Highway

$0-5$

$6-10$

$11-15$

16-20

$21-30$

$31-40$

$41-50$

51 or more
Age Group

Middle-Aged

16.7

8.3

16.7

16.7

8.3

8.3

25.0 $\underline{\text { Older }}$

22.2

22.2

16.7

16.7

5.6

16.7

Percentage of drivers by type of vehicle driven as a function of age

$\begin{array}{lccc}\text { Type of Vehicle } & \text { Young } & \frac{\text { Age Group }}{\text { Middle-Aged }} & \\ \begin{array}{l}\text { Passenger Car } \\ \text { Motor home/RV }\end{array} & 91.7 & 75.0 & \text { Older } \\ \begin{array}{l}\text { Motorcycle } \\ \text { Light Truck / Van }\end{array} & - & & - \\ \begin{array}{l}\text { Heavy Truck } \\ \text { Bus }\end{array} & -8.3 & 25.0 & 11.1\end{array}$

\section{Stimuli And Apparatus}

Acuity was assessed using a custom Landolt $C$ acuity chart, at a luminance of $110 \mathrm{~cd} / \mathrm{m}^{2}$. As in the previous study, contrast sensitivity was measured at spatial frequencies of 1.5, 3.0, 6.0, 12.0 , and $18.0 \mathrm{c} / \mathrm{deg}$ and a luminance of $135 \mathrm{~cd} / \mathrm{m}^{2}$. Participants were refracted to best acuity at test distance on both the acuity and contrast sensitivity measures. The average correction for best acuity at $83 \mathrm{~cm}$ (32.6 in), in absolute values, was $0.10 \mathrm{D}$ for the young, $0.27 \mathrm{D}$ for the middle aged, and $0.65 \mathrm{D}$ for the older. Refractions were carried out with an American Optical Master Phoropter; optical corrections were implemented using a R.H. Burton trial lens set with lens clip or trial frame. 
The Y SYMBOL sign (MUTCD No. W2-1) was used to demonstrate the task. The test stimuli consisted of the same 18 symbol signs tested in the Glance Legibility/Reaction Time studies. As described in the glance legibility and reaction time study, rank, or daytime legibility, and comprehension were used jointly to select six Best, six Intermediate, and six Worst signs. To assure that signs were comfortably above their minimum legibility size for all participants, their screen sizes were scaled to simulate a "real-world" viewing distance of $26.6 \mathrm{~m}(87.3 \mathrm{ft})$ at the test distance. The resulting screen height of each sign is presented in appendix F.

All signs were presented in their regulation colors on an Apple High-Resolution $330 \mathrm{~mm}$ (13.in) color monitor, driven by a Raster Ops 364 24-bit video board under control of a Macintosh IIci computer. White-area screen luminance was maintained at $77 \mathrm{~cd} / \mathrm{m}^{2}$ using a Minolta LS-110 spot photometer. To maintain a constant viewing distance, participants viewed the signs from a chin rest. Each trial began with a 2 -s presentation of a centrally located fixation cross $1.8 \mathrm{~cm}(0.7 \mathrm{in})$ or $1.23^{\circ}$ wide by $1.8 \mathrm{~cm}(0.7 \mathrm{in})$ or $1.23^{\circ}$. A 'target' sign was then presented for $2 \mathrm{~s}$, followed by a distracter array of 17 signs made up from the balance of the test set (16 distracters and the target sign on target-present trials, 17 distracters on target-absent trials). A distracter array consisting of the signs themselves was utilized so that the signs could be compared directly to one another under similar conditions and because any other single background would favor the conspicuity of some signs over others. Observers indicated whether a target sign was present in the array or not by pressing the "Present" or "Absent" keys respectively, located on a control console built over the computer's keyboard; pressing the correct key initiated the next trial.

The distracter array consisted of a 4 vertical by 5 horizontal position matrix yielding 20 possible distracter sign positions, and occupied a window $12.2^{\circ}$ vertically by $16.2^{\circ}$ horizontally. To allow for target-absent trials, only 17 of the 20 positions could be used on any single trial. The location of the filled and empty positions, as well as the specific signs within the distracter matrix, varied randomly from trial to trial. The location of the target sign within the matrix on target-present trials changed randomly from trial to trial with the condition that any given sign message never appeared twice in the same row or column. The specific sign that was omitted from the distracter array on target-present trials was counterbalanced across the 18 signs. As a result, no two arrays were identical. Each sign was presented as a target six times; on three trials, it was present in the array and on three it was not.

\section{Procedure}

Following the administration of the driving questionnaire, the assessment of acuity and contrast sensitivity, and refraction to the test distance, the conspicuity search task was explained to the participants. They were then introduced to the task using the Y SYMBOL practice sign. Next, they were familiarized with the 18 signs in the test set by viewing them one at a time, in random order with the sign names above them. At the beginning, subjects were instructed to emphasize accuracy (i.e., to strike the correct key) while responding as quickly as possible. The search time taken to indicate correctly whether or not the target sign was present, as well as the accuracy of response, were both recorded by the computer. Search times in excess of $10 \mathrm{~s}$ were deemed to be lapses of attention and were recorded by the computer as $10 \mathrm{~s}$. The test trials were divided into six blocks, each of 18 randomly ordered trials (1 per sign), for a total of 108 trials. The same 6 random orders were used for all participants. A brief rest period of a minute or two 
(as determined by the participant), was provided between each block. The presentation rate of the signs was the same for each subject. All testing occurred in a single session. Session lengths for the young, middle-aged, and older participants, respectively, were $54 \mathrm{~min}, 59 \mathrm{~min}$, and 68 $\mathrm{mm}$.

\section{RESULTS}

\section{Contrast Sensitivity}

Since a mixed design Age (3) x Gender (2) x Spatial Frequency (5) ANOVA on the logtransformed contrast sensitivity data showed no significant effect for gender, subsequent analyses were carried out collapsing across this variable. An Age x Spatial Frequency ANOVA indicated significant effects for age, $[\mathrm{F}(2,39)=12.78, \underline{p}<0.001]$, spatial frequency $[\underline{F}(4,156)=70.77$, $\underline{\mathrm{p}}<0.001]$, and their interaction $[\underline{\mathrm{F}}(8,156)=7.77, \mathrm{p}<0.001]$. As can be seen in figure 13 , as spatial frequency increased, the contrast sensitivity deficit was greater for older drivers. The mean log contrast sensitivity functions (CSF's) of the age groups were either close to or within the VCTS 6000 population norms for observers aged 10 to 70 years; sensitivity of the young observers exceeded the norms slightly at $6.0 \mathrm{c} / \mathrm{deg}$. Tukey HSD pair-wise comparisons (with Tukey-Kramer adjustment for unequal n's) showed significantly greater age differences at higher spatial frequencies. No age differences occurred at 1.5 or $3.0 \mathrm{c} / \mathrm{deg}$, but at $6.0,12.0$ and 18.0 $\mathrm{c} / \mathrm{deg}$ the sensitivity of the young exceeded that of both the middle-aged and older groups. There were no significant sensitivity differences between the middle-aged and older drivers.

As in the previous studies, the utility of a single CSF-derived score for predicting conspicuity thresholds was determined using several composite measures derived from individual log CSF's: peak sensitivity, peak spatial frequency, and high cutoff. Because the CSF assessed by the VCTS did not fall below half of its peak value on the low-spatial frequency end for several observers, it was not possible to derive low cutoff or bandwidth (i.e., high cutoff-low cutoff) measures from them. As can be seen in table 31, Tukey pairwise comparisons revealed a significant age deficit on the peak sensitivity and peak spatial frequency measures, but not the high-cutoff measure.

Table 31. Age differences in derived contrast sensitivity measures.

\begin{tabular}{|c|c|c|c|c|}
\hline & Young & $\begin{array}{c}\text { Middle- } \\
\text { Aged }\end{array}$ & $\underline{\text { Older }}$ & Age Diff \\
\hline Peak Sensitivity (Log CS) & 2.36 & 2.13 & 2.08 & $a b$ \\
\hline Peak Spatial Frequency (c/deg) & 6.50 & 5.00 & 5.58 & $a b$ \\
\hline High Cutoff (c/deg) & 11.71 & 11.22 & 9.95 & - \\
\hline
\end{tabular}

Notes:

$\mathrm{a}=$ Significant difference $(\mathrm{p}<.05$ or better) between Young vs. Older Drivers.

$\mathrm{b}=$ Significant difference $(\mathrm{p}<.05$ or better) between Young vs. Middle-Aged Drivers. 


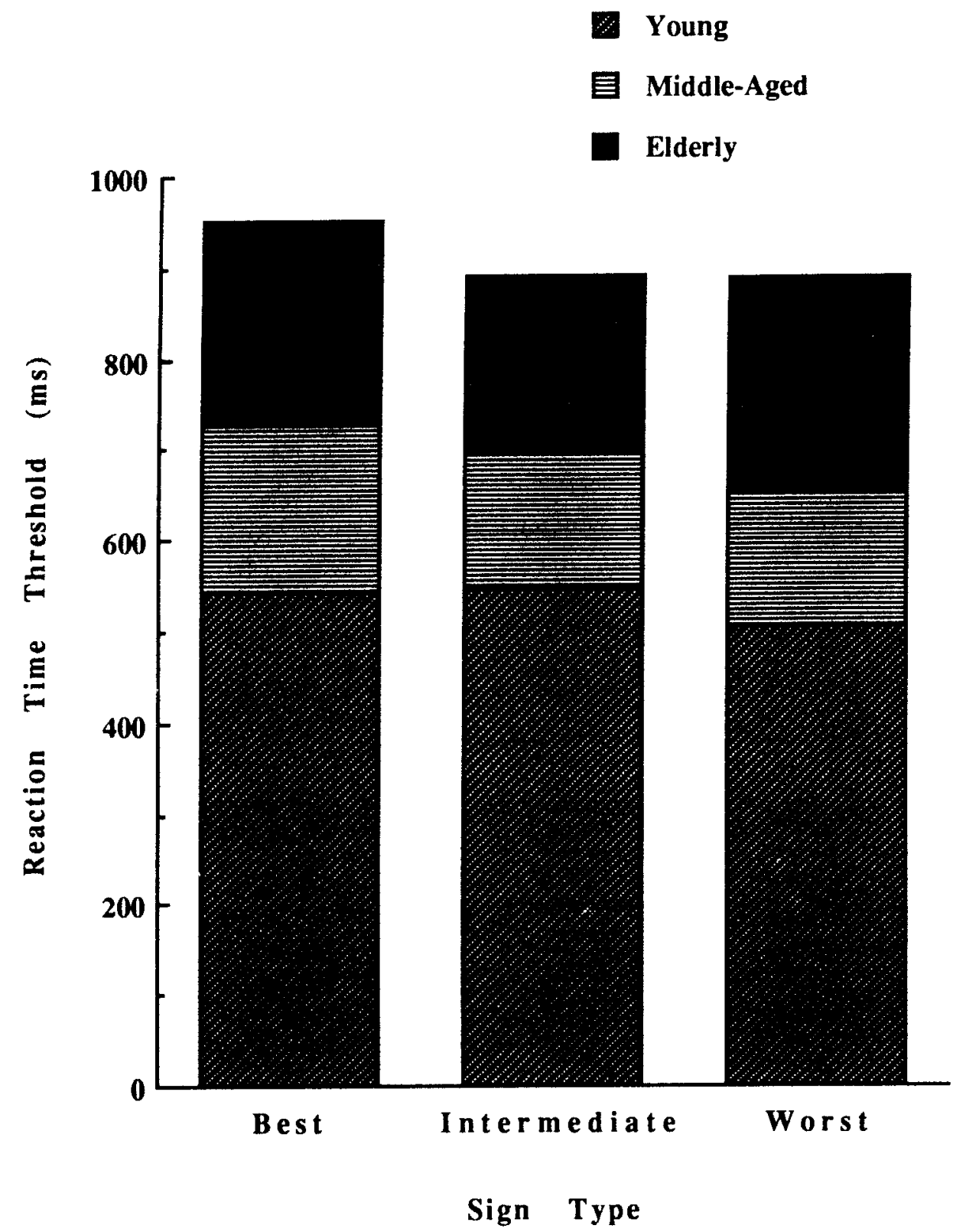

Figure 12. Reaction time of best, intermediate, and worst symbol signs among young, middle-aged, and elderly drivers. 


\section{Search Time}

An Age Group (3) by Gender (2) by Sign (18) mixed design ANOVA, on conspicuity search time thresholds, revealed no gender effects, so it was excluded from further analyses.

Differences due to age and signs were then assessed in an Age (3) by Sign (18) mixed design ANOVA. It showed significant effects for age $[\underline{F}(2,39)=10.53, \underline{p}<0.001]$, sign $[\underline{F}(17,663)=$ 17.8 ,

$\mathrm{p}<0.001]$, and their interaction $[\mathrm{F}(34,663)=1.98, \mathrm{p}<0.01]$. Mean conspicuity thresholds for each sign and age group, are presented in table 32, and also graphically in figure 14. As can be seen there, there were marked differences in the search times for different signs and increased driver age was associated with longer search times.

A Sign Category (3) by Age (3) by Target Present/Absent (2) mixed design ANOVA on the search time data revealed significant effects for age $[\underline{F}(2,198)=118.4, p<.001]$, for target present/absent $[\underline{F}(1,198)=34.54, \underline{p}<.001]$, and a significant interaction between them $[\underline{F}(2,198)=7.38, \underline{p}<.001]$. Tukey pairwise comparisons revealed that the presence or absence of the target did not affect the young or middle aged subjects, but among the older, search times were significantly longer on target-absent trials $(\mathrm{p}<.001)$ (See figure 15). None of the possible effects for sign category (Best, Intermediate and Worst) were significant.

\section{Response Accuracy}

Differences in accuracy between signs for each age group were evaluated in a Sign (18) by Age (3) mixed design ANOVA. A significant effect was observed for signs $[\underline{F}(17,663)=$ 4.94, $\mathrm{p}<.001]$, as well as a significant interaction between age and $\operatorname{sign}[\underline{\mathrm{F}}(34,663)=1.54, \mathrm{p}<.05]$. Mean percent correct first responses for each sign and age group are presented in table 33. Age differences were found on only two signs.

Age differences in the effects on accuracy of the target sign being present or absent, as a function of sign category were analyzed in an Age (3) by Sign Category (3) by Target Present/Absent (2) mixed-design ANOVA. It revealed that response accuracy was greater on trials when the target was present than when it was absent $[\underline{F}(1,198)=65.57, p<.001]$, but no significant effects for age or sign type or their possible interactions. Pearson correlation between overall percent correct and mean search time per sign revealed, as expected, a significant relationship $(\underline{r}=-.52, \underline{p}<.001)$. This relationship between response speed and response accuracy was also significant within each age group: for the young, $\underline{r}=-.54, \underline{p}<.05$, for the middle-aged, $\underline{r}=-.60, p<.01$, and for the older, $\underline{r}=-.87, \underline{p}<.001$. R to Fisher 2 transformations were carried out but showed no significant differences between the age groups in the strength of the accuracy/search time relationship, although the difference between the older and the young drivers approached significance $(\mathrm{p}=.052)$. 
Table 32. Mean conspicuity and standard deviations (SD) in ms of 18 symbol signs among young, middle-aged, and older drivers, ranked from lowest to highest on overall mean.

\begin{tabular}{|c|c|c|c|c|c|c|}
\hline$\underline{\text { Rank }}$ & $\begin{array}{l}\text { Sign Name } \\
\text { (MUTCD No.) }\end{array}$ & $\begin{array}{l}\text { Age } \\
\text { Diff }\end{array}$ & $\frac{\text { Young }}{(\mathrm{SD})}$ & $\begin{array}{l}\text { Middle- } \\
\frac{\text { Aged }}{\underline{(\mathrm{SD})}}\end{array}$ & $\frac{\text { Older }}{\underline{(\mathrm{SD})}}$ & $\begin{array}{c}\begin{array}{c}\text { All } \\
\text { Groups }\end{array} \\
\frac{(\mathrm{SD})}{}\end{array}$ \\
\hline 1. & $\begin{array}{l}\text { Advance Flagger } \\
\text { (W20-7a) }\end{array}$ & $\mathrm{ac}$ & $\begin{array}{c}948.9 \\
(198.0)\end{array}$ & $\begin{array}{l}1187.1 \\
(307.9)\end{array}$ & $\begin{array}{l}1630.2 \\
(747.5)\end{array}$ & $\begin{array}{l}1255.4 \\
(599.4)\end{array}$ \\
\hline 2. & $\begin{array}{l}\text { Div Hwy Ends } \\
\text { (W6-2) }\end{array}$ & $\mathrm{a}$ & $\begin{array}{l}1259.2 \\
(253.7)\end{array}$ & $\begin{array}{l}1349.2 \\
(228.1)\end{array}$ & $\begin{array}{l}1597.2 \\
(586.8)\end{array}$ & $\begin{array}{l}1401.9 \\
(448.9)\end{array}$ \\
\hline 3. & $\begin{array}{l}\text { Phone } \\
\text { (D9-1) }\end{array}$ & $\mathrm{a}$ & $\begin{array}{l}1157.9 \\
(338.9)\end{array}$ & $\begin{array}{l}1416.8 \\
(541.5)\end{array}$ & $\begin{array}{l}1844.7 \\
(965.8)\end{array}$ & $\begin{array}{l}1473.1 \\
(772.7)\end{array}$ \\
\hline 4. & $\begin{array}{l}\text { Cross Road } \\
\text { (W2-1) }\end{array}$ & $\mathrm{a}$ & $\begin{array}{l}1188.4 \\
(247.1)\end{array}$ & $\begin{array}{l}1470.1 \\
(386.4)\end{array}$ & $\begin{array}{l}1827.9 \\
(838.3)\end{array}$ & $\begin{array}{l}1495.5 \\
(656.6)\end{array}$ \\
\hline 5. & $\begin{array}{l}\text { Hospital } \\
\text { (D9-2) }\end{array}$ & $\mathrm{a}$ & $\begin{array}{l}1069.2 \\
(187.8)\end{array}$ & $\begin{array}{l}1477.7 \\
(653.9)\end{array}$ & $\begin{array}{c}2079.7 \\
(1442.2)\end{array}$ & $\begin{array}{c}1542.2 \\
(1089.3)\end{array}$ \\
\hline 6. & $\begin{array}{l}\text { Bicycle Crossing } \\
\text { (W11-1) }\end{array}$ & $\mathrm{a}$ & $\begin{array}{l}1230.2 \\
(251.1)\end{array}$ & $\begin{array}{l}1648.4 \\
(766.0)\end{array}$ & $\begin{array}{l}1876.7 \\
(938.8)\end{array}$ & $\begin{array}{l}1585.1 \\
(792.2)\end{array}$ \\
\hline 7. & $\begin{array}{l}\text { Pavement Ends } \\
\text { (W8-3a) }\end{array}$ & $\mathrm{a}$ & $\begin{array}{l}1120.4 \\
(294.8)\end{array}$ & $\begin{array}{l}1770.2 \\
(76 \quad 1.2)\end{array}$ & $\begin{array}{c}1877.8 \\
(1049.4)\end{array}$ & $\begin{array}{l}1589.5 \\
(925.1)\end{array}$ \\
\hline 8. & $\begin{array}{l}\text { Campfire } \\
(\mathrm{Ra}-030)\end{array}$ & $\mathrm{a}$ & $\begin{array}{l}1227.5 \\
(244.0)\end{array}$ & $\begin{array}{l}1498.1 \\
(396.3)\end{array}$ & $\begin{array}{c}2232.4 \\
(1201.5)\end{array}$ & $\begin{array}{l}1652.7 \\
(925.1)\end{array}$ \\
\hline 9. & $\begin{array}{l}\text { Right Curve } \\
\text { (W1-2R) }\end{array}$ & $\mathrm{ac}$ & $\begin{array}{l}1316.6 \\
(335.1)\end{array}$ & $\begin{array}{l}1641.5 \\
(521.7)\end{array}$ & $\begin{array}{c}2036.3 \\
(1222.6)\end{array}$ & $\begin{array}{l}1664.8 \\
(916.4)\end{array}$ \\
\hline 10. & $\begin{array}{l}\text { RV Sanitary Stn } \\
\text { (D9-12) }\end{array}$ & $\mathrm{ac}$ & $\begin{array}{l}1299.5 \\
(176.3)\end{array}$ & $\begin{array}{l}1569.1 \\
(372.5)\end{array}$ & $\begin{array}{c}2272.3 \\
(1250.4)\end{array}$ & $\begin{array}{l}1713.7 \\
(982.7)\end{array}$ \\
\hline 11. & $\begin{array}{l}\text { Ranger Station } \\
(\mathrm{RG}-170)\end{array}$ & $\mathrm{ac}$ & $\begin{array}{l}1409.7 \\
(287.6)\end{array}$ & $\begin{array}{l}1567.5 \\
(372.5)\end{array}$ & $\begin{array}{c}2534.0 \\
(1250.4)\end{array}$ & $\begin{array}{l}1837.1 \\
(982.7)\end{array}$ \\
\hline
\end{tabular}

Notes:

$\mathrm{a}=$ Significant difference $(\mathrm{p}<.05$ or better) between Young and Older Drivers

$\mathrm{c}=$ Significant difference $(\mathrm{p}<.05$ or better $)$ between Middle-Aged and Older Drivers 
Table 32. Mean conspicuity and standard deviations (SD) in ms of 18 symbol signs among young, middle-aged, and older drivers, ranked from lowest to highest on overall mean (continued).

\begin{tabular}{|c|c|c|c|c|c|c|}
\hline$\underline{\operatorname{Rank}}$ & $\begin{array}{l}\text { Sign Name } \\
\text { (MUTCD No.) }\end{array}$ & $\begin{array}{l}\text { Age } \\
\text { Diff }\end{array}$ & $\frac{\text { Young }}{(\mathrm{SD})}$ & $\begin{array}{l}\text { Middle } \\
\frac{\text { Aged }}{(\mathrm{SD})}\end{array}$ & $\frac{\text { Older }}{(\mathrm{SD})}$ & $\begin{array}{c}\text { All } \\
\text { Groups } \\
\underline{(\mathrm{SD})}\end{array}$ \\
\hline 12. & $\begin{array}{l}\text { Skating } \\
(\mathrm{RS}-010)\end{array}$ & $\mathrm{ac}$ & $\begin{array}{l}1344.9 \\
(240.6)\end{array}$ & $\begin{array}{l}1866.6 \\
(445.4)\end{array}$ & $\begin{array}{c}2347.5 \\
(1267.8)\end{array}$ & $\begin{array}{l}1853.0 \\
(976.4)\end{array}$ \\
\hline 13. & $\begin{array}{l}\text { Mandatory Seat Belt } \\
\text { (R16-1) }\end{array}$ & $\mathrm{a}$ & $\begin{array}{l}1302.6 \\
(292.4)\end{array}$ & $\begin{array}{l}1747.6 \\
(995.7)\end{array}$ & $\begin{array}{c}2528.0 \\
(1090.0)\end{array}$ & $\begin{array}{c}1859.4 \\
(1029.9)\end{array}$ \\
\hline 14. & $\begin{array}{l}\text { Keep Right } \\
\text { (R4-7) }\end{array}$ & $\mathrm{ac}$ & $\begin{array}{r}1331.0 \\
(370.0)\end{array}$ & $\begin{array}{r}1759.0 \\
(840.2)\end{array}$ & $\begin{array}{c}2759.1 \\
(1563.1)\end{array}$ & $\begin{array}{c}1949.7 \\
(1283.1)\end{array}$ \\
\hline 15 . & $\begin{array}{l}\text { No Parking } \\
\text { (R8-3a) }\end{array}$ & $\mathrm{a}$ & $\begin{array}{l}1479.6 \\
(454.8)\end{array}$ & $\begin{array}{l}2188.9 \\
(513.1)\end{array}$ & $\begin{array}{c}2482.0 \\
(1123.2)\end{array}$ & $\begin{array}{l}2050.2 \\
(915.9)\end{array}$ \\
\hline 16. & $\begin{array}{l}\text { No Trucks } \\
\text { (R5-2) }\end{array}$ & $\mathrm{ac}$ & $\begin{array}{l}1649.5 \\
(411.4)\end{array}$ & $\begin{array}{l}1765.6 \\
(383.3)\end{array}$ & $\begin{array}{c}2782.4 \\
(1344.5)\end{array}$ & $\begin{array}{c}2065.8 \\
(1060.0)\end{array}$ \\
\hline 17. & $\begin{array}{l}\text { No U-Turn } \\
\text { (R3-4) }\end{array}$ & $\mathrm{ac}$ & $\begin{array}{l}1594.6 \\
(430.3)\end{array}$ & $\begin{array}{l}1955.9 \\
(541.4)\end{array}$ & $\begin{array}{c}2759.6 \\
(1228.5)\end{array}$ & $\begin{array}{c}2103.3 \\
(1011.5)\end{array}$ \\
\hline 18. & $\begin{array}{l}\text { National Network } \\
\text { Route Prohibited } \\
\text { (R14-5) }\end{array}$ & $\mathrm{a}$ & $\begin{array}{l}1613.1 \\
(318.5)\end{array}$ & $\begin{array}{l}2083.8 \\
(838.0)\end{array}$ & $\begin{array}{c}2694.4 \\
(1341.3)\end{array}$ & $\begin{array}{c}2130.4 \\
(1088.5)\end{array}$ \\
\hline & Overall Mean & & 1307.9 & 1664.6 & 2231.2 & 1734.6 \\
\hline & Overall SD & & 188.9 & 256.9 & 400.1 & 259.7 \\
\hline
\end{tabular}


Table 33. Mean percent first response correct and standard deviations (SD) of 18 symbol signs among young, middle-aged, and older drivers, ranked from highest to lowest on overall mean.

\begin{tabular}{|c|c|c|c|c|c|c|}
\hline$\underline{\text { Rank }}$ & $\begin{array}{c}\begin{array}{c}\text { Sign Name } \\
\text { (MUTCD No.) }\end{array} \\
\end{array}$ & $\begin{array}{l}\text { Age } \\
\text { Diff }\end{array}$ & $\frac{\text { Young }}{\underline{(\mathrm{SD})}}$ & $\frac{\text { iddle A }}{\underline{(\mathrm{SD})}}$ & $\frac{\text { Older }}{\underline{(\mathrm{SD})}}$ & $\frac{\frac{\text { All }}{\text { Groups }}}{\text { (SD) }}$ \\
\hline 1. & Div Hwy Ends & $\mathrm{ab}$ & 90.3 & 100.0 & $\overline{100.0}$ & 97.2 \\
\hline & (W6-2) & & (13.2) & $(0.00)$ & $(0.00)$ & $(8.2)$ \\
\hline 2. & $\begin{array}{l}\text { Cross Road } \\
\text { (W2-1) }\end{array}$ & & $\begin{array}{l}97.2 \\
(6.5)\end{array}$ & $\begin{array}{c}91.7 \\
(11.2)\end{array}$ & $\begin{array}{l}98.2 \\
(5.4)\end{array}$ & $\begin{array}{l}96.0 \\
(8.1)\end{array}$ \\
\hline 3. & $\begin{array}{l}\text { Hospital } \\
\text { (D9-2) }\end{array}$ & & $\begin{array}{l}97.2 \\
(9.6)\end{array}$ & $\begin{array}{l}97.2 \\
(6.5)\end{array}$ & $\begin{array}{c}94.4 \\
(11.4)\end{array}$ & $\begin{array}{l}96.0 \\
(9.6)\end{array}$ \\
\hline 4. & $\begin{array}{c}\text { Campfire } \\
(\mathrm{Ra}-030)\end{array}$ & - & $\begin{array}{l}98.6 \\
(4.8)\end{array}$ & $\begin{array}{l}95.8 \\
(7.5)\end{array}$ & $\begin{array}{c}92.6 \\
(10.3)\end{array}$ & $\begin{array}{l}95.2 \\
(8.5)\end{array}$ \\
\hline 5. & $\begin{array}{l}\text { Mandatory Seat Belt } \\
\text { (R16-1) }\end{array}$ & & $\begin{array}{l}98.6 \\
(4.8)\end{array}$ & $\begin{array}{c}94.4 \\
(10.9)\end{array}$ & $\begin{array}{c}92.6 \\
(10.3)\end{array}$ & $\begin{array}{l}94.8 \\
(9.4)\end{array}$ \\
\hline 6. & $\begin{array}{l}\text { P h o n e } \\
\text { (D9-1) }\end{array}$ & & $\begin{array}{c}94.4 \\
(10.9)\end{array}$ & $\begin{array}{l}97.2 \\
(9.6)\end{array}$ & $\begin{array}{l}93.5 \\
(10.1)\end{array}$ & $\begin{array}{c}94.8 \\
(10.1)\end{array}$ \\
\hline 7. & $\begin{array}{l}\text { Right Curve } \\
\text { (W1-2R) }\end{array}$ & & $\begin{array}{c}95.8 \\
(10.4)\end{array}$ & $\begin{array}{l}98.6 \\
(4.9)\end{array}$ & $\begin{array}{c}91.7 \\
(13.1)\end{array}$ & $\begin{array}{c}94.8 \\
(10.7)\end{array}$ \\
\hline 8. & $\begin{array}{l}\text { Advance Flagger } \\
\text { (W20-7a) }\end{array}$ & & $\begin{array}{l}93.1 \\
(8.6)\end{array}$ & $\begin{array}{l}95.8 \\
(7.5)\end{array}$ & $\begin{array}{l}94.9 \\
(7.7)\end{array}$ & $\begin{array}{l}94.6 \\
(7.8)\end{array}$ \\
\hline 9. & $\begin{array}{l}\text { Bicycle Crossing } \\
\text { (W11-1) }\end{array}$ & & $\begin{array}{l}93.1 \\
(8.6)\end{array}$ & $\begin{array}{l}94.4 \\
(8.2)\end{array}$ & $\begin{array}{l}95.4 \\
(9.6)\end{array}$ & $\begin{array}{l}94.4 \\
(8.8)\end{array}$ \\
\hline
\end{tabular}

Note:

$\mathrm{a}=$ Significant difference $(\mathrm{p}<.05$ or better) between Young and Older Drivers.

$\mathrm{b}=$ Significant difference $(\mathrm{p}<.05$ or better) between Young and Middle-Aged.

$\mathrm{c}=$ Significant difference $(\mathrm{p}<.05$ or better) between Middle-Aged and Older Drivers. 
Table 33. Mean percent first response correct and standard deviations (SD) of 18 symbol signs among young, middle-aged, and older drivers, ranked from highest to lowest on overall mean (continued).

\begin{tabular}{|c|c|c|c|c|c|c|}
\hline \multirow{3}{*}{$\underline{\text { Rank }}$} & $\underline{\text { Sign Name }}$ & Age & \multicolumn{3}{|c|}{$\underline{\text { Middle }}$} & \multirow{2}{*}{$\begin{array}{c}\text { All } \\
\text { Group } \\
\end{array}$} \\
\hline & (MUTCD No.) & Diff & $\underline{\text { Young }}$ & $\underline{\text { Aged }}$ & $\underline{\text { Older }}$ & \\
\hline & & & $(\mathrm{SD})$ & $(\mathrm{SD})$ & $\underline{(\mathrm{SD})}$ & $\underline{(\mathrm{SD})}$ \\
\hline \multirow[t]{2}{*}{10.} & Pavement Ends & & 94.4 & 94.4 & 92.6 & 93.7 \\
\hline & (W8-3a) & & $(8.2)$ & $(8.2)$ & $(10.3)$ & $(9.0)$ \\
\hline \multirow[t]{2}{*}{11.} & Skating & & 90.3 & 97.2 & 93.5 & 93.7 \\
\hline & (RS-010) & & $(13.2)$ & $(9.6)$ & $(10.1)$ & $(11.0)$ \\
\hline \multirow[t]{2}{*}{12 . } & No Parking & & 95.8 & 91.7 & 92.6 & 93.3 \\
\hline & (RS-3a) & & $(7.5)$ & (8.7) & $(13.1$ & $(10.5)$ \\
\hline \multirow[t]{2}{*}{13.} & Ranger Station & & 93.1 & 95.8 & 90.7 & 92.9 \\
\hline & (RG-170) & & $(13.2)$ & $(10.4)$ & (8.5) & $(10.5)$ \\
\hline \multirow[t]{2}{*}{14} & Keep Right & & 94.4 & 95.8 & 89.8 & 92.9 \\
\hline & (R4-7) & & $(10.9)$ & (7.5) & $(13.0)$ & $(11.1)$ \\
\hline \multirow[t]{2}{*}{15} & RV Sanitary Stn & & 90.3 & 95.8 & 91.7 & 92.5 \\
\hline & (D9-12) & & $(13.2)$ & $(7.5)$ & $(11.8)$ & $(11.2)$ \\
\hline \multirow[t]{2}{*}{16.} & No Trucks & $\mathrm{c}$ & 87.5 & 97.2 & 84.3 & 88.9 \\
\hline & (R5-2) & & $(14.4)$ & $(6.5)$ & $(14.5$ & $(13.6)$ \\
\hline \multirow[t]{2}{*}{17.} & No U-Turn & & 88.9 & 86.1 & 83.3 & 85.7 \\
\hline & (R3-4) & & $(10.9)$ & $(12.0)$ & $(17.2)$ & $(14.1)$ \\
\hline \multirow[t]{4}{*}{18} & National Network & & & & & \\
\hline & $\begin{array}{l}\text { Route Prohibited } \\
\text { (R14-5) }\end{array}$ & & $\begin{array}{c}88.9 \\
(17.9)\end{array}$ & $\begin{array}{c}76.4 \\
(20.7)\end{array}$ & $\begin{array}{c}85.2 \\
(16.1)\end{array}$ & $\begin{array}{c}83.7 \\
(18.2)\end{array}$ \\
\hline & Overall Mean & & 93.4 & 94.2 & 92.1 & 93.2 \\
\hline & Overall SD & & 3.5 & 5.4 & 4.3 & 3.5 \\
\hline
\end{tabular}




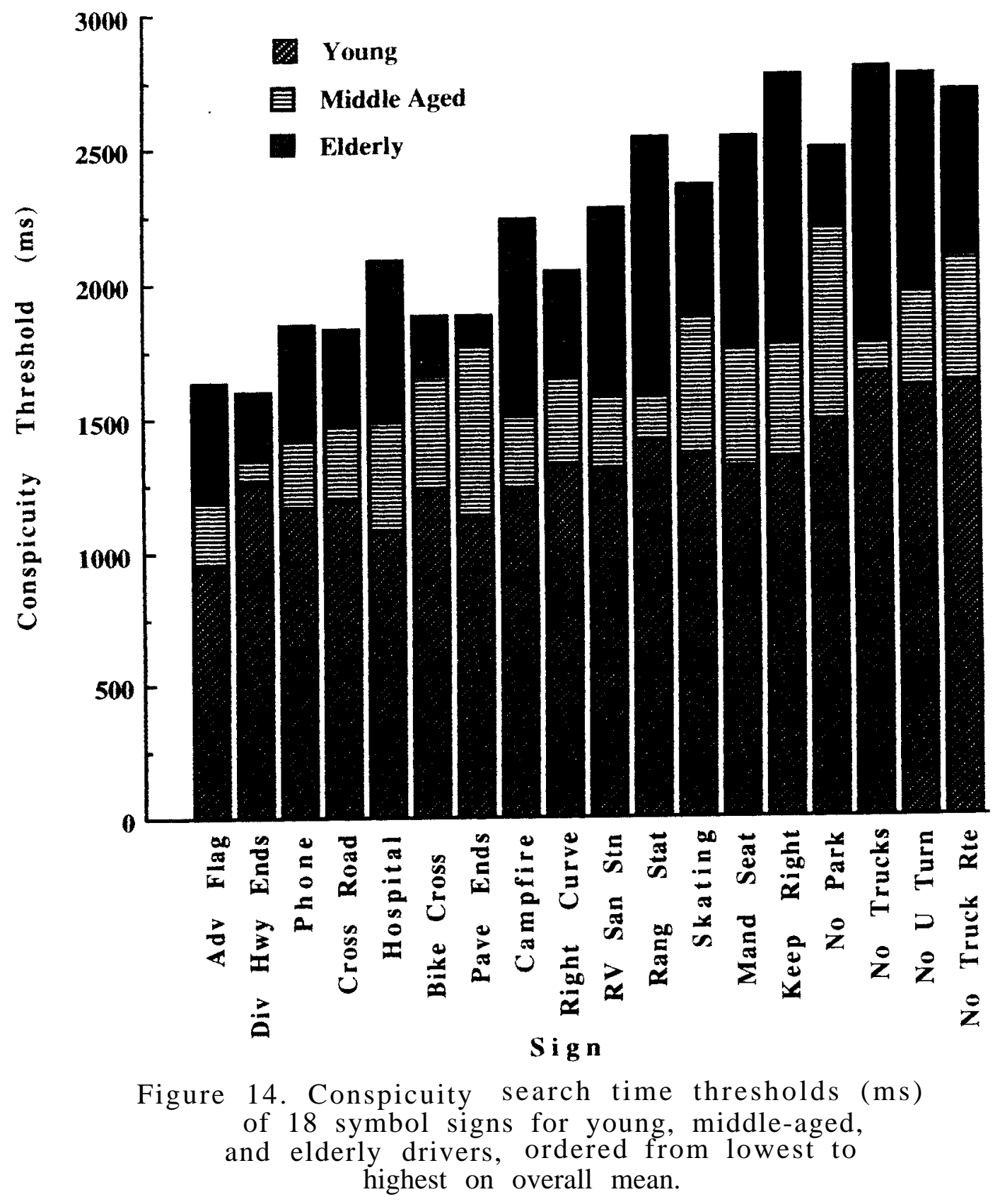




\section{Relationships Between Visual Measures And Conspicuity}

Pearson product-moment correlations were calculated to determine the relationships between conspicuity search time threshold on one hand, and the visual measures of best acuity, peak contrast sensitivity, high cutoff, and peak spatial frequency on the other, both across the age groups, and separately within each age group. Acuity and conspicuity threshold were moderately related across the three age groups $(\underline{r}=0.62, p<0.001)$, as well as within the young $(\underline{r}=0.59$, $\mathrm{p}<0.05)$ group. There was no relationship between acuity and conspicuity among middle-aged ( $\underline{\mathrm{r}}=-0.12, \underline{\mathrm{p}}=0.72)$, or older $(\underline{\mathrm{r}}=0.43, \underline{\mathrm{p}}=0.07)$ drivers. Transformation of these correlations to Fisher's Z scores, revealed no significant differences between the age groups in the strength of the acuity/conspicuity search time relationship. Of the three contrast sensitivity measures, only peak sensitivity correlated significantly with conspicuity thresholds when assessed across age ( $\underline{r}=-0.46, p<.05)$. Within each age group, however, none of the measures showed a meaningful relationship with conspicuity.

\section{Relationships Between Conspicuity And Other Measures Of Sign Effectiveness}

To evaluate possible relationships between the various measures used to evaluate the effectiveness of the 18 signs in the test set, Spearman correlations were calculated between their rankings on search time in the present study, and those in earlier studies in this series: day legibility, comprehension, night legibility, night legibility with glare, and glance legibility/reaction time. Conspicuity was significantly related only to night legibility $(\underline{r}=.48, \underline{p}$ $<.05)$. The relationship between day legibility and conspicuity approached significance $(\underline{r}=.44$, $\mathrm{p}<.10)$.

\section{DISCUSSION}

\section{Conspicuity}

As expected, conspicuity search times for different symbol signs varied widely. The search time for the worst sign in the test set (NATIONAL NETWORK ROUTE PROHIBITED), for example, was about 70 percent greater than that of the best sign (ADVANCE FLAGGER). Much of this difference appeared to be attributable to similarities between the signs in the test set rather than to particular properties of signs themselves (see table 32). For example, the three of the five top ranked signs (DIVIDED HIGHWAY ENDS, CROSS ROAD and HOSPITAL were very different from one another in size, appearance and color. The three signs at the bottom of the list (NO TRUCKS, NO U TURN, NATIONAL NETWORK PROHIBITED), however, were all black-on-white regulatory signs with the prohibitory red circle and diagonal slash; two of them also shared different silhouette views of a truck. Presumably, conspicuity search times of any one of these three signs would have been lower if the other two were not in the test set. This presumption raises the issue of the validity of these data, since in the "real world" drivers are not normally compelled to search through 17 signs to find the one they need. This problem, however, would have been increased rather than reduced by the use of a more naturalistic background distracter array, since it would inevitably favor the conspicuity of some signs over others

(e.g., white signs are more conspicuous against a green background than against snow). That in turn, would make a comparison of different signs meaningless, except on a context-specific 
basis, a problem reduced in the present study by using signs as their own controls in the distracter array. Thus, the present findings are in keeping with those from earlier studies showing that a sign's conspicuity is in large degree a function of its contrast with its background. Although, it would be possible to maximize the "pop out" value of signs for the context in which they are located, that would be completely impractical since that would mean changing the color of signs for different locations, or even seasons.

Older drivers were slower than their young and middle-aged counterparts in detecting signs. In fact, they exhibited greater search times than young drivers for every one of the 18 signs tested. Not surprisingly, the age difference was greater on target-sign-absent trials where the search process is more extensive, since it cannot be terminated until it has covered the entire distracter array at least once (see figure 15). Response accuracy and search time were related for all three age groups. This is not surprising in that a response error necessarily extends search time. Only modest age differences involving two signs, however, were seen on accuracy of response. On one sign, the difference favored older and middle-aged drivers (DIVIDED HIGHWAY ENDS), and on the other, middle-aged ones over the older (NO TRUCKS). These findings suggest that older drivers maximize their accuracy by extending their visual search time.

\section{Conspicuity And Visual Functioning}

Although the acuity level of the older participants was better than average for their age, consistent with prior research, their acuity was below to that of younger observers. ${ }^{(170)}$ The increasing age deficit in contrast sensitivity at higher spatial frequencies was also consistent with the pattern that has been observed in previous studies. ${ }^{(14,125)}$

Search conspicuity performance was clearly not a function of observers' spatial visual limitations, nor was it expected to be. The search conspicuity task involved detecting signs that were high in contrast and many times larger than their legibility threshold. Although poor acuity was associated with longer search times when calculated across all age groups, it simply reflected that both measures declined with age. When calculated within age groups to determine the utility of acuity as a predictor of search time conspicuity regardless of driver age, it was significant only among young drivers. The measures of contrast sensitivity were even more weakly related to search time than was acuity-none of the contrast measures were useful predictors for any age group. Given that they appear to provide a better estimate of the difficulties observers have in identifying and localizing peripherally presented complex stimuli, a useful field of view measure would probably be suitable for predicting search conspicuity among drivers of different age. ${ }^{(174)}$

\section{Relationships Between Conspicuity And Other Measures}

The available evidence suggested that search conspicuity is a relatively unique measure, weakly related to legibility, and not at all related to measures such as comprehension, glance legibility, or reaction time. One implication of this finding is that, in the design process, conspicuity must be treated as a unique issue related to a sign's color and features in combination with its placement and the characteristics of the environment in which it is located. The dependence of conspicuity on such contextual variables means that it is not a very useful measure for guiding or evaluating symbol sign design. 


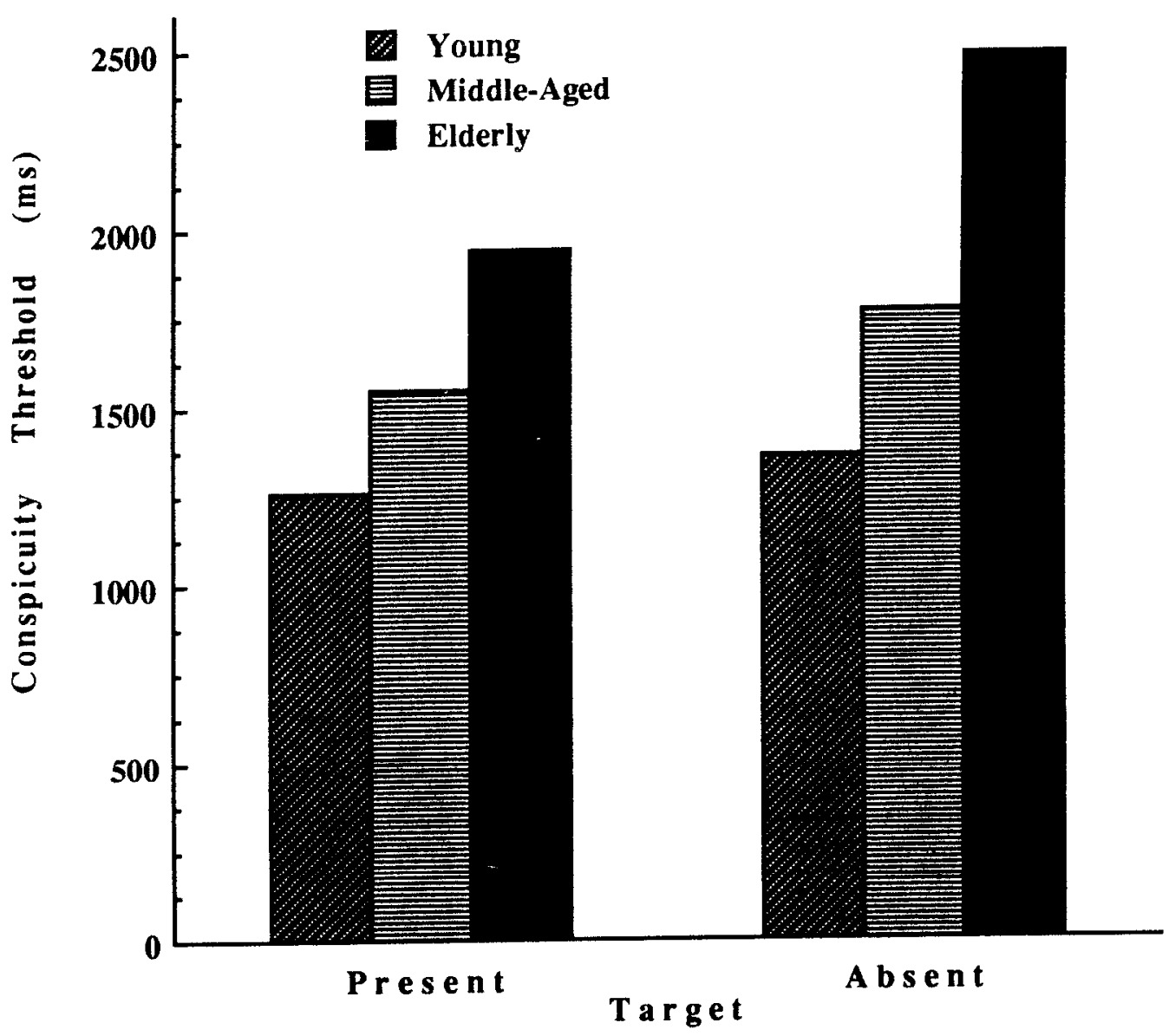

Figure 15. Conspicuity search time thresholds (ms) for young, middle-aged, and elderly drivers

as a function of target-sign-present and target-sign-absent trials. 



\section{CHAPTER 9: DEVELOPMENT OF ALTERNATIVE AND NOVEL SYMBOL SIGNS}

A major objective of this project was to develop a procedure for improving existing symbols and designing new ones for future use. Depending on their initial comprehension levels and legibility, improvements to existing symbols were accomplished through one of two basic processes. Modifications, intended primarily to improve legibility distances, were carried out on signs with good levels of comprehension. For signs with poor comprehension and legibility, a two-stage redesign process, intended to enhance signs on both dimensions, was carried out. In addition, five completely novel symbol messages were designed, using the redesign approach.

The specific procedures for the modification or redesign of current signs, as well as for the creation of novel symbols, are described below. Following the development of these revised and novel symbols, they were evaluated for comprehension and legibility distance under day, night, and night-with-glare conditions.

The legibility distance of all three sign types was optimized by using an image-processing reiterative filtering technique to guide feature design (see Design Approach section). To estimate the gradient for improving signs standard signs that differed in their initial legibility and comprehensibility, six of the seven Modified signs, as well as six of the seven Redesigned signs, were selected from among the six Best, six Intermediate, and six Worst signs in terms of legibility and comprehensibility as determined in the previous studies of comprehension and legibility. This was the same set of 18 signs that was evaluated with regard to night with glare, glance legibility and reaction time, and conspicuity. This set included two Warning, Regulatory, and Guide/Information signs in each quality category. Based on their importance to driver safety, all 6 of the Warning signs from the original set of 18 (i.e., the two Best, two Intermediate, and two Worst) were either Modified $(\mathrm{N}=3)$ or Redesigned $(\mathrm{N}=3)$; among Regulatory and Guide/Information signs, one each of the Best, Intermediate, and Worst signs was Modified or Redesigned (see appendix H). Signs in the Best category were Modified given that they were already highly effective (CROSS ROAD, RIGHT CURVE, KEEP RIGHT, HOSPITAL), signs from the Worst category were Redesigned (PAVEMENT ENDS, BICYCLE CROSSING, MANDATORY SEATBELT, RANGER STATION), and signs from the Intermediate category were either Modified (i.e., NO PARKING-letter P, DIVIDED HIGHWAY ENDS) or Redesigned (i.e., ADVANCE FLAGGER, CAMPFIRE). A seventh, not from the test set of 18 (LANE REDUCTION TRANSITION), was included as a Modified sign at the request of the FHWA. The seventh Redesigned sign, also not from the 18 sign test set, was a new pictographic version of the No Parking sign designed by the investigators, which used a rear view silhouette of a car and curb rather than the letter "P" to convey its message. The five Novel signs: RESTRICTED VISIBILITY, CROSS WINDS, SCHOOL BUS STOP AHEAD, HORSE DRAWN VEHICLES, and TRUCK ENTRANCE, were selected by the FHWA and conveyed messages not currently in the Manual. 


\section{MODIFICATION OF SIGNS}

To maintain their high legibility and comprehension levels, the spatial format of the Best and Intermediate signs from the 18 signs were modified rather than redesigned. In the modification process, the legibility of signs was enhanced using an image-processing reiterative filter/redesign approach (see figure 16). Gray-scale versions of each sign were digitized and stored in an IBM AT computer using a Matrox PIP-1024 image processing board and a Panasonic CCTV model WV- 1410 video camera. The resulting digitized images were filtered using a sequence of low-pass order 2 (i.e., 12dB/octave) Butterworth filters of progressively lower cut-offs (i.e., every even-numbered harmonic). The resulting filtered images were examined to determine the lowest filter cut-off level at which all of its details as defined by the comprehension scoring key (see appendix E) were preserved. Features which became indistinct or which interacted negatively with adjacent features at any given cut-off level were modified, and the digitizing/filtering process repeated. Typically, this involved increasing the size of details to make them more legible, or changing their shape and/or location to diminish contour interactions with adjacent details. This process was carried out reiteratively until no further improvements in a sign could be discerned. For example, legibility of the DIVIDED HIGHWAY ENDS sign (see figure 17), was increased by making the directional arrows longer, and by reducing the width and altering the shape of the barrier around which the arrows flow.

\section{REDESIGN OF SIGNS}

Signs with low initial legibility and comprehension levels were subjected to a two-stage redesign process. In the first stage, a professional graphics design team was briefed by lab personnel, both on the specific limitations of signs tested in prior studies, as well as on the spatial characteristics of signs that enhance their legibility (i.e., large simple contours, wide contour separations). The importance of creating signs that could withstand low-pass filtration by maximizing their contour size, simplicity and separation, and by avoiding the interaction of adjacent contours, was stressed using examples of "good" and "bad" signs. The effectiveness of this approach was enhanced by having the designer optimize the legibility of each feature when the sign was blurred by a strong positive sphere $(5.5 \mathrm{D})$ lens. The resulting designs were viewed by the lab staff and suggestions for further improvement were offered through two successive iterations. In the second and final design stage, black and white versions of the first-stage designs were digitized into the computer and reiteratively filtered to increase their legibility using the same procedure described in the modification process above. The changes that resulted from this process are exemplified by the redesigned PAVEMENT ENDS sign. The current manual version of the sign presents an aerial view of a striped and homogeneously black (paved) road surface juxtaposed with one composed of asymmetrical spots to indicate a "rough road" ahead. These spots, however, are so small that they severely limit the legibility of the sign. To avoid this limitation, the redesigned version (see figure 18) shows a side view of a car, the front end of which is over a chunky and undulating road surface. Even when the relatively large chunks that compose the rough part of the road are at a distance too great to be discriminated individually, or are blurred due to poor acuity, the undulations created by their vertically offset locations signal that the surface ahead is a rough one. 


\section{DESIGN OF NOVEL SIGNS}

To maximize the comprehension level of each of the Novel signs, the design team selected the most promising of the various concepts provided by the graphics designer. This initial concept was then refined by the designer over two successive design stages based on input provided by the design team. The legibility of the resulting display was then optimized using the image-processing approach described in the preceding Redesign section. For example, on the CROSS WINDS sign, to indicate that the car was being tilted by the force of air and not some other medium (e.g., such as water from a hydrant) the "wind lines" were revised so that they both rose over the car. The legibility of the sign (figure 19) was then maximized by simplifying the car design, by increasing the thickness of the contours defining the car, wind lines and road surface, and finally, by increasing the separation of the wind lines. 


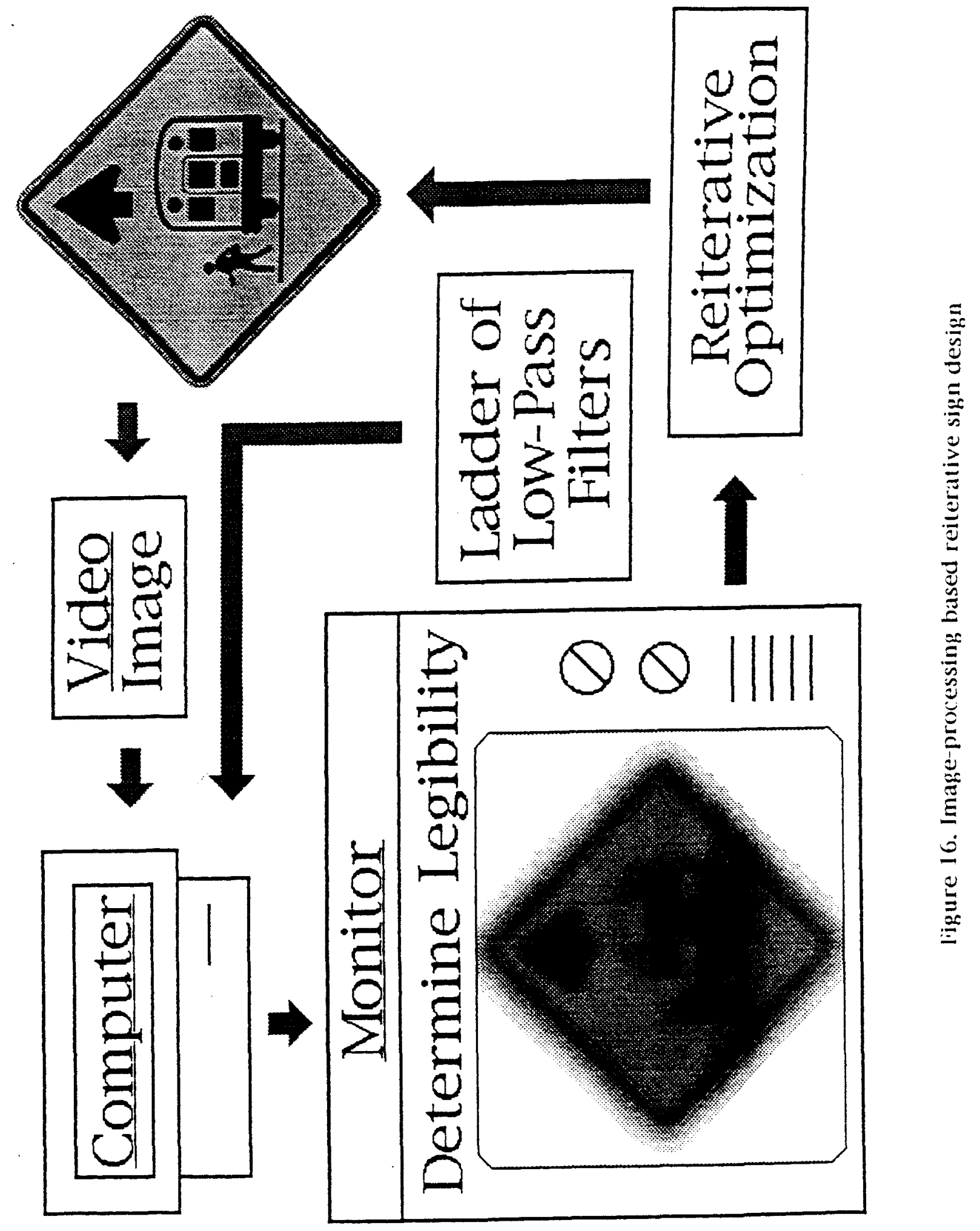




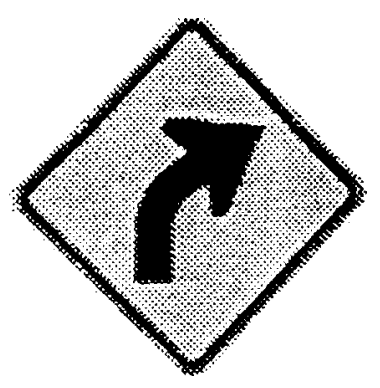

Right Curve

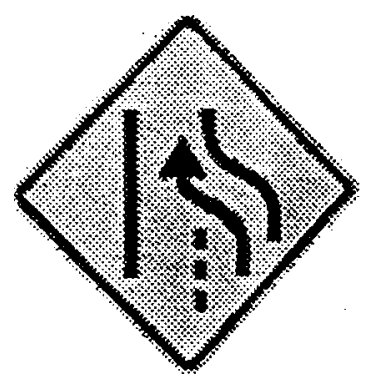

Lane Reduction

Transition

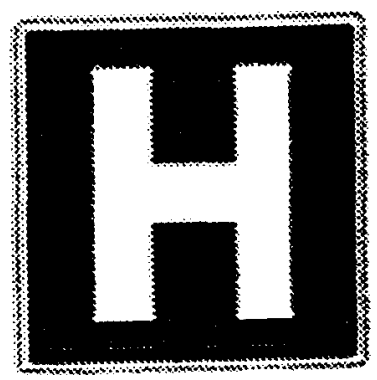

Hospital

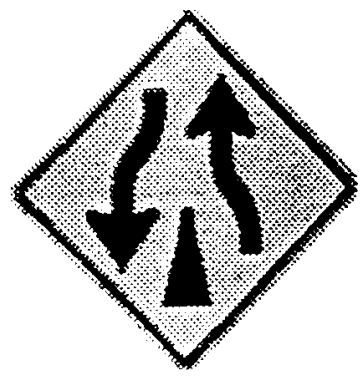

Divided Highway

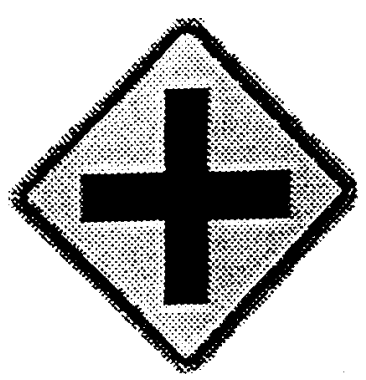

Cross Road

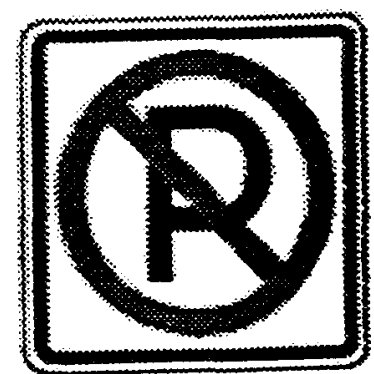

No Parking

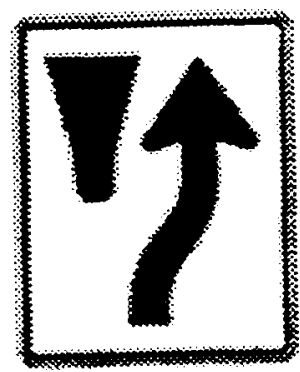

Keep Right

Figure 17. Modified Signs. 


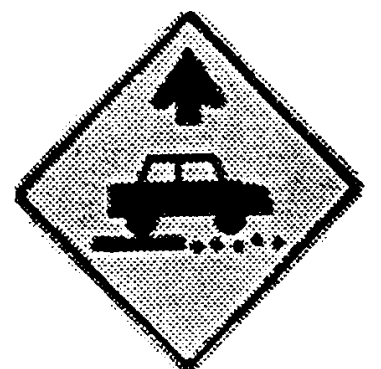

Pavement Ends

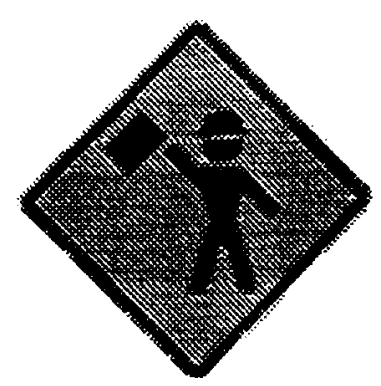

Advance Flagger

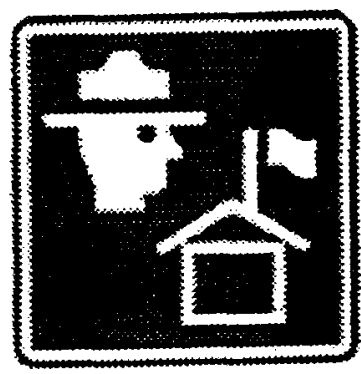

Ranger Station

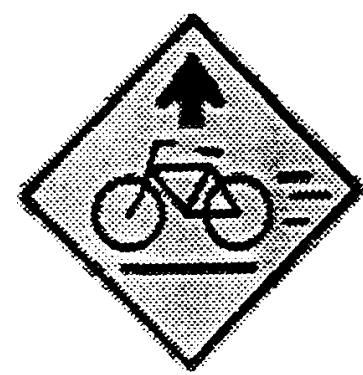

Bicycle Crossing

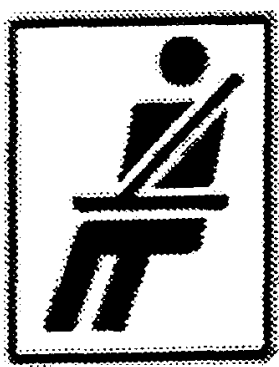

-Mandatory Seat Belts

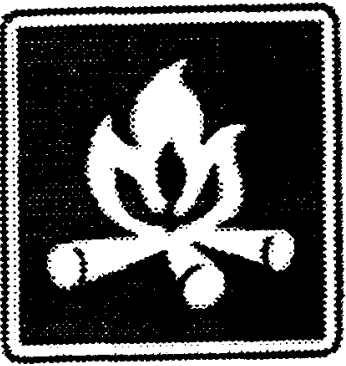

Campfire

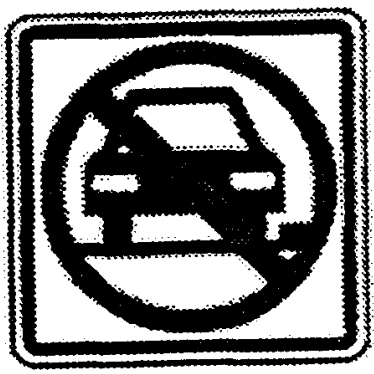

No Parking

Figure 18. Redesigned Signs. 


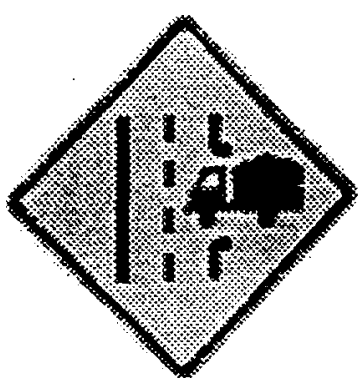

Truck Entrance

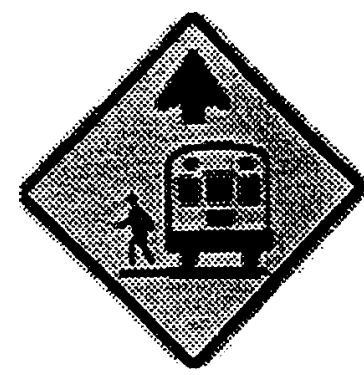

School Bus

Stop Ahead

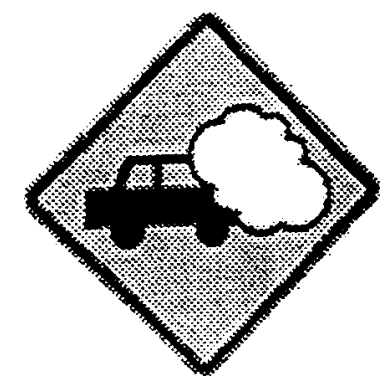

Reduced Legibility

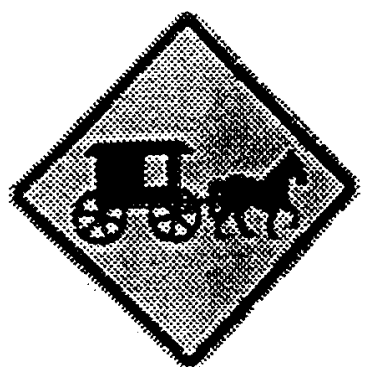

Horse Drawn

Vehicles

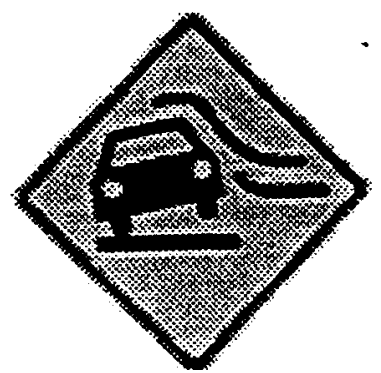

Cross Winds

Figure 19. Novel Signs. 


\section{CHAPTER 10: COMPREHENSION OF MODIFIED, REDESIGNED, AND NOVEL SYMBOLS}

\section{FOCUS GROUP STUDY}

The objective of phase 2 of the project was to examine differences in the level of understanding, and the day, night, and night-with-glare legibility of revised versions of 13 of the symbols tested in the preceding studies, as well as the understanding and legibility of symbol versions of five novel symbol messages.

A small sample of older drivers were interviewed to determine their views on the modified and novel symbols. In order to obtain these general impressions drivers over the age of 60 were interviewed using a focus group procedure, modified slightly from that used in phase 1.

\section{METHOD}

\section{Subjects}

Seventeen volunteer drivers, ( 9 females and 8 males) over the age of 60 , one group of six in Boise, ID, and two groups ( $\mathrm{N}=5$ and 6$)$ in Calgary, $\mathrm{AB}$, participated.

\section{Procedure}

The procedure was essentially the same as that used in the previous focus group discussions, where small groups of older drivers were asked to discuss a series of issues related to traffic signs, with the emphasis being on the effectiveness of symbol messages. In addition to the discussion of questions posed in the initial focus group sessions, the revised versions of 13 messages, as well as five novel symbols (black and white photocopies on letter-size paper), were shown to the subjects and they were asked to write out their meanings on an answer sheet. Following this, the original versions of these messages were displayed simultaneously with the new versions and subjects were asked for their views on their relative effectiveness. Discussion was directed toward the relative understandability and legibility of the symbols. The procedure required about less than $2 \mathrm{~h}$ and subjects were given an honorarium of $\$ 20$ for their participation.

\section{RESULTS}

The primary objective of these focus group discussions was to determine older drivers' impressions of the new designs. Therefore, the general discussion about traffic signs will only be described briefly. Specific difficulties with traffic signs included their poor conspicuity at locations where there are several other signs (visual overload or clutter) and difficulty in seeing them at night. However, the Idaho group felt that symbol signs were more easily detected in visual clutter than were word signs.

The general views about traffic sign symbols were that they are easier to notice, can be understood at a greater distance, and more quickly read than word signs. The subjects felt that the design of symbols should be simple, with few details, and with bolder, solid (not outline) figures. It was mentioned that not all messages can be depicted with symbols. 
The following observations were made about the differences between the original and the revised symbols:

KEEP RIGHT-This symbol was well understood; some thought the new version of the divider was a bit better, but in general there was seen to be very little difference between the two.

NO PARKING-The new version was thought by several to look more like a P, but subjects were split in terms of whether it should be changed.

MANDATORY SEAT BELT-The original torso was thought by some to look too fat, but that the original symbol had a more realistic looking Seat belt. Both versions were thought to look like a wheelchair, and views were divided as to whether the new one was visually simpler.

RIGHT CURVE-No differences were noticed between the two versions of this message.

CROSS ROAD-No differences were noticed between the two versions, and two people commented that the original was fine.

DIVIDED HIGHWAY ENDS-No differences were noticed, but some thought the new version might be better for drivers with poor vision and better at night.

PAVEMENT ENDS-Understanding was reasonably good, and it was considered a good representation of the ending of a paved roadway surface. Rough or gravel road ahead was considered by some to be an equivalent message.

BICYCLE CROSSING-The new version was generally understood, but some still thought it meant bicycle path. The addition of a line under the bicycle and speed lines behind the bicycle got mixed reviews. Some thought these helped convey the idea of a crossing, while others said it made no difference.

ADVANCE FLAGGER-Impressions of the new version were quite positive. Modifications to the hat were considered to help distinguish the figure from a pedestrian and a school crossing guard and to convey the idea of construction zone better. One subject suggested the hand with the flag would be better if straight out, rather than upward, to depict more effectively the idea of stop (as used in hand signaling on the railways).

HOSPITAL-No differences were seen by any subjects, and it was felt there was no need to change the symbol.

RANGER STATION-The new version was generally liked, but many still thought it could mean police station. Modifications to the hat and head were seen as good, as were changes to the building.

CAMPFIRE-Impressions of the new version were generally negative and understanding was poor. The main observation was that the original design of the logs was better. The older version was thought to be less complex and to look more like a fire. 
LANE REDUCTION TRANSITION-The symbol was liked by several subjects and reasonably well understood, but the new version was considered to be visually complex, and did not give a clear indication that the lane ends. One group was unanimous in saying that the older version was better.

NO PARKING (pictograph)--Understanding was poor, and it was considered to be a poor sign, with many subjects thinking it meant no stopping, no entry, or no cars allowed. The presence of vehicle lights made it look like the front, not the rear, of a car, and made it seem that the car was in operation, which would fail to depict "no parking." The small size of the curb made it difficult to see.

Comments on the novel symbols were as follows:

REDUCED VISIBILITY-Understanding was good, and it was felt that the cloud depicted fog well. Suggestions for changes included the word FOG in the cloud and increasing the size of the cloud. Concern was expressed about the use of the sign and the possibility for false alarms (e.g., would it be used when no fog was present?).

CROSS WINDS-It was felt that this was a good symbol for cross winds and that the lines did suggest wind. However, -it was pointed out that wind does not overturn cars, and that a truck, RV or van should be the vehicle used.

SCHOOL BUS STOP AHEAD-The bus symbol was considered by the two Alberta groups to be a clear indication of a school bus-the details conveyed this idea, but were not too complex to recognize. This positive response may have been due to the fact that a similar symbol is used on Alberta roads. Some of the Idaho group did not understand it to be a school bus.

HORSE DRAWN VEHICLES-This sign was well understood and liked. There was a strong association with groups, such as Amish and Hutterites, who use these vehicles. The horse was felt to be a good representation of this animal.

TRUCK ENTRANCE-The message was well understood by the Alberta sample, again, possibly because a variation of this symbol is used on Canadian roads. It was felt that the solid line on the left indicated that the trucks would be turning, not going straight across the road. Orientation of the sign was deemed by some to be important in order to indicate the direction from which the trucks would be entering the road.

General-Acceptance of the new and the revised symbols was generally good, and they were fairly well understood, with a couple of exceptions. In addition, the arrow at the top of some signs (PAVEMENT ENDS, BICYCLE CROSSING, SCHOOL BUS STOP AHEAD) was well understood as indicating that the hazard was ahead up the road.

\section{COMPREHENSION}

This component of phase 2 evaluated the relationship between driver age and level of understanding of the Modified, Redesigned, and Novel symbols and compared understanding on the revised versions with that on the original MUTCD versions tested in phase 1. 


\section{METHOD}

\section{Subjects}

The subjects were volunteer licensed drivers, aged 18 years and older in Texas, Idaho, and Alberta, Canada. These subjects were solicited in the same manner as for described in chapter 4--from existing subject pools (in Texas and Alberta), local service clubs, newspaper ads, recreational/social organizations for senior citizens, and through personal contact by the experimenters. The breakdown of subjects by age, gender, and location is shown in table 34 .

The demographic data for annual distance driven, percentage of night driving, driving environment, and vehicle type are shown in table 35 . It can be seen that these are very similar to the corresponding data from phase 1 comprehension study sample.

\section{Stimuli And Apparatus}

The stimuli were 19 color slides of traffic sign symbols. Thirteen of these were revised versions of symbols in the 1988 edition of the MUTCD, four were symbol versions of novel messages (not in the Manual as either word or symbol signs) and one, SCHOOL BUS STOP AHEAD, was a symbol version of an existing word message. In addition, a novel version of the NO PARKING message was evaluated to determine the adequacy of a pictograph in place of the upper case P. The LANE REDUCTION TRANSITION" sign was not one of the 12 selected on the basis of data from phase 1, but was redesigned and evaluated at the request of FHWA. The specific messages evaluated can be found in table 36, and are illustrated in figures 17, 18, and 19.

A Kodak Carousal slide projector (Model 4000) was used to project the slides. The screens on which the images were projected varied in size, depending on the dimensions of the testing room. Test booklets consisting of eight pages were used for subjects to provide the background information requested and to write their responses. These test booklets were similar to those used in the initial comprehension study, with the exception of the number of signs (19 instead of 86).

Approximately equal numbers of drivers were tested in each age by gender by location combination at each location. The age categories were 18 to 39, 40 to 59, 60 to 69, and 70 and above. The major variable of interest was age.

\section{Procedure}

The procedure was essentially identical to that used in the previous comprehension study, with the exception that there was no break half way through the testing (this was deemed not to be necessary, as the entire procedure took less than an hour). Subjects viewed each slide for 30 to $40 \mathrm{~s}$ and were required to write the meaning of each in the appropriate place in the answer booklet, and to indicate on a five-point scale how familiar they were with each symbol. Participants were given an honorarium of $\$ 10$. 
Table 34. Number of participants as a function of location and age: comprehension (phase 2).

\begin{tabular}{|c|c|c|c|c|c|c|c|}
\hline \multirow[t]{2}{*}{ Age } & \multicolumn{2}{|c|}{$\underline{\text { Texas }}$} & \multicolumn{2}{|c|}{$\underline{\text { Idaho }}$} & \multicolumn{2}{|c|}{ Alberta } & Totals \\
\hline & Male & Female & Male & Female & Male & Female & \\
\hline $18-39$ & 11 & 12 & 12 & 12 & 12 & 13 & 72 \\
\hline $40-59$ & 13 & 12 & 12 & 12 & 11 & 12 & 72 \\
\hline $60-69$ & 6 & 6 & 5 & 6 & 7 & 7 & 37 \\
\hline $70+$ & 7 & 5 & 7 & 6 & 7 & 6 & 38 \\
\hline Totals & 37 & 35 & 36 & 36 & 37 & 38 & 219 \\
\hline Site Totals & 7 & 2 & 72 & & & 5 & \\
\hline
\end{tabular}

Table 35. Participant demographics: comprehension (phase 2).

Percentage of drivers by distance driven past 12 months as a function of age Age Group

Distance driven

\begin{tabular}{|c|c|c|c|c|}
\hline (miles) & 18 to 39 & 40 to 59 & $\underline{60 \text { to } 69}$ & $\underline{70+}$ \\
\hline Under 5,000 & 25 & 22 & 27 & 37 \\
\hline $5,000-9,999$ & 23 & 19 & 42 & 34 \\
\hline $10,000-14,999$ & 22 & 26 & 18 & 21 \\
\hline $15,000-19,999$ & 15 & 16 & 10 & 7 \\
\hline 20,000 or more & 15 & 17 & 3 & 1 \\
\hline
\end{tabular}

Percentage of drivers by night driving as a function of age

Percentage miles

\begin{tabular}{|c|c|c|c|c|}
\hline driven at night & 18 to 39 & 40 to 59 & 60 to 69 & $\underline{70+}$ \\
\hline $0-5$ & 3 & 17 & 27 & 36 \\
\hline $6-10$ & 7 & 15 & 31 & 23 \\
\hline $11-15$ & 12 & 15 & 15 & 11 \\
\hline $16-20$ & 19 & 20 & 13 & 15 \\
\hline $21-30$ & 21 & 14 & 7 & 7 \\
\hline $31-40$ & 18 & 9 & 5 & 5 \\
\hline $41-50$ & 16 & 7 & 2 & 3 \\
\hline 51 or more & 4 & 3 & 0 & 0 \\
\hline
\end{tabular}


Table 35. Participant demographics: comprehension (phase 2) (continued).

Percentage of drivers by driving environment as a function of age

Driving

Age Group

\begin{tabular}{|c|c|c|c|c|}
\hline Environment & 18 to 39 & 40 to 59 & 60 to 69 & $\underline{70+}$ \\
\hline Rural/sparsely pop. & 9 & 7 & 6 & 9 \\
\hline Small town & 2 & 3 & 2 & 5 \\
\hline Suburbs of city & 52 & 47 & 45 & 48 \\
\hline Inner city & 37 & 43 & 47 & 38 \\
\hline
\end{tabular}

$\underline{\text { Percentage of drivers by type of vehicle driven as a function of age }}$

Age Group

\begin{tabular}{|c|c|c|c|}
\hline Type of Vehicle & 18 to 39 & 40 to 59 & $\underline{60 \text { to } 69}$ \\
\hline Passenger Car & 80 & 74 & 77 \\
\hline Motor home/RV & 1 & 0 & 0 \\
\hline Motorcycle & 19 & 24 & 23 \\
\hline Light Truck/Van & 0 & 0 & 0 \\
\hline Heavy Truck & 0 & 0 & 0 \\
\hline Bus & 0 & 2 & 0 \\
\hline $\mathrm{Cab}$ & 0 & 0 & 0 \\
\hline
\end{tabular}

\section{RESULTS AND DISCUSSION}

The scoring procedure was identical to that used in the phase 1 comprehension study, and all data were scored by the same research assistant who did it for that study, ensuring a high level of consistency in the data reduction. To establish reliability both the research assistant and a Principal Investigator scored the novel symbol data of several subjects from each location and age group. Agreement among the judges was very high. The scoring criteria for those messages previously tested in the first comprehension study were as used in that study. Criteria for the novel signs are shown in appendix I.

The frequency of each response made to each sign, as a function of age and gender, is shown in table 36. For the purpose of data analysis, responses scored as correct and partially correct (2 and 1) were combined. The mean percent correct, as a function of age, for the new symbols are given in table 37. Although those symbols most poorly understood in the earlier study were also poorly understood in this study, improvements were achieved for three of the messages. On the other hand, some symbols were more poorly understood as can be seen in table 38, which shows the mean percent correct for standard and new signs. The former are included for ease of comparison with the understandability levels for the original designs. It can be seen that comprehension of the new designs is high for those messages that were well understood in the first comprehension study (NO PARKING, RIGHT CURVE, CROSS ROAD, HOSPITAL), while it was still generally poor for those not previously well understood. The percentage correct comprehension of each sign category as a function of age is shown in table 39 and figure 20. 
Table 36. Number of responses to each symbol as a function of age and gender.

\begin{tabular}{|c|c|c|c|c|c|c|c|c|}
\hline \multirow{3}{*}{$\frac{\text { Sign Name }}{\text { Response** }^{* *}}$} & \multicolumn{8}{|c|}{$\underline{\text { Modified Signs }}$} \\
\hline & \multirow[t]{2}{*}{$\underline{\text { MUTCD\# }}$} & \multirow{2}{*}{$\underline{A g e}^{*}$} & \multicolumn{3}{|c|}{$\underline{\text { Males }}$} & \multicolumn{3}{|c|}{$\underline{\text { Females }}$} \\
\hline & & & 0 & 1 & 2 & 0 & 1 & 2 \\
\hline \multirow[t]{4}{*}{ Right Curve } & \multirow[t]{4}{*}{ W 1 - 2 R } & $\mathrm{Y}$ & 2 & 14 & 19 & 3 & 16 & 18 \\
\hline & & M & 0 & 10 & 26 & 2 & 10 & 24 \\
\hline & & 0 & 0 & 7 & 11 & 3 & 10 & 6 \\
\hline & & $\mathrm{E}$ & 3 & 13 & 5 & 0 & 11 & 6 \\
\hline \multirow[t]{4}{*}{ No Parking } & \multirow[t]{4}{*}{ R $8-3 a$} & $\mathrm{Y}$ & 0 & 0 & 35 & 0 & 0 & 37 \\
\hline & & M & 2 & 1 & 33 & 1 & 1 & 34 \\
\hline & & 0 & 1 & 0 & 17 & 3 & 0 & 16 \\
\hline & & $\mathrm{E}$ & 5 & 0 & 16 & 2 & 0 & 15 \\
\hline \multirow[t]{4}{*}{ Hospital } & \multirow[t]{4}{*}{ D $9-2$} & $\mathrm{Y}$ & 1 & 0 & 34 & 2 & 1 & 34 \\
\hline & & M & 3 & 0 & 33 & 3 & 0 & 33 \\
\hline & & 0 & 2 & 0 & 16 & 2 & 0 & 17 \\
\hline & & $\mathrm{E}$ & 4 & 1 & 16 & 2 & 0 & 15 \\
\hline \multirow[t]{4}{*}{ Cross Road } & \multirow[t]{4}{*}{ W 2 - 1} & $\mathrm{Y}$ & 1 & 0 & 34 & 6 & 1 & 30 \\
\hline & & M & 1 & 0 & 35 & 3 & 1 & 32 \\
\hline & & 0 & 0 & 2 & 16 & 7 & 1 & 11 \\
\hline & & $\mathrm{E}$ & 8 & 0 & 13 & 7 & 2 & 8 \\
\hline \multirow[t]{4}{*}{ Keep Right } & \multirow[t]{4}{*}{ R 4 - 7} & Y & 1 & 11 & 23 & 7 & 16 & 14 \\
\hline & & M & 2 & 14 & 20 & 5 & 14 & 17 \\
\hline & & 0 & 1 & 7 & 10 & 3 & 5 & 11 \\
\hline & & $\mathrm{E}$ & 7 & 7 & 7 & 8 & 2 & 7 \\
\hline \multirow{4}{*}{$\begin{array}{l}\text { Divided } \\
\text { Highway } \\
\text { Ends }\end{array}$} & \multirow[t]{4}{*}{ W 6 - 2} & Y & 11 & 4 & 20 & 11 & 8 & 18 \\
\hline & & M & 14 & 4 & 18 & 18 & 8 & 10 \\
\hline & & 0 & 4 & 3 & 11 & 10 & 4 & 5 \\
\hline & & E & 10 & 3 & 8 & 10 & 2 & 5 \\
\hline Lane & \multirow[t]{4}{*}{ W 4 - 2} & $\mathrm{Y}$ & 7 & 12 & 16 & 8 & 10 & 19 \\
\hline Reduction & & M & 16 & 9 & 11 & 16 & 5 & 15 \\
\hline \multirow[t]{2}{*}{ Transition } & & 0 & 4 & 7 & 7 & 14 & 2 & 3 \\
\hline & & E & 13 & 2 & 6 & 14 & 2 & 1 \\
\hline Mandatory & R 16-1 & $\mathrm{Y}$ & 0 & 0 & 35 & 1 & 1 & 35 \\
\hline \multirow[t]{3}{*}{ Seat Belts } & & M & 1 & 0 & 35 & 5 & 0 & 31 \\
\hline & & 0 & 2 & 0 & 16 & 5 & 0 & 14 \\
\hline & & $\mathrm{E}$ & 9 & 0 & 12 & 8 & 0 & 9 \\
\hline Advance & $\mathrm{W} 20-7 \mathrm{a}$ & $\mathrm{Y}$ & 2 & 4 & 29 & 6 & 12 & 19 \\
\hline \multirow[t]{3}{*}{ Flagger } & & M & 5 & 3 & 28 & 6 & 7 & 23 \\
\hline & & 0 & 1 & 3 & 14 & 5 & 0 & 14 \\
\hline & & $\mathrm{E}$ & 7 & 1 & 13 & 8 & 1 & 8 \\
\hline
\end{tabular}

*Age: $\mathrm{Y}=18$ to $39 ; \mathrm{M}=40$ to $59 ; \mathrm{O}=60$ to $69 ; \mathrm{E}=70+$

**Response: $0=$ incorrect; $1=$ partially correct; $2=$ correct 
Table 36. Number of responses to each symbol as a function of age and gender (continued).

$\underline{\text { Redesigned Signs }}$

\begin{tabular}{|c|c|c|c|c|c|c|c|c|}
\hline Sign Name & MUTCD\# & $\mathrm{Age}^{*}$ & & Iale & & & ma & \\
\hline$\widetilde{\text { Response }^{* *}}$ & & & 0 & 1 & 2 & 0 & 1 & 2 \\
\hline Ranger & $\mathrm{RG}-170$ & Y & 3 & 4 & 28 & 4 & 6 & 27 \\
\hline Station & & M & 10 & 4 & 22 & 15 & 5 & 16 \\
\hline & & 0 & 10 & 0 & 8 & 8 & 3 & 8 \\
\hline & & $\mathrm{E}$ & 12 & 1 & 8 & 10 & 2 & 5 \\
\hline Campfire & RA- 030 & $\mathrm{Y}$ & 13 & 4 & 18 & 6 & 1 & 30 \\
\hline & & M & 8 & 2 & 26 & 7 & 3 & 26 \\
\hline & & 0 & 12 & 0 & 6 & 13 & 0 & 6 \\
\hline & & $\mathrm{E}$ & 15 & 0 & 6 & 11 & 0 & 6 \\
\hline Pavement & W $8-3 a$ & $\mathrm{Y}$ & 12 & 3 & 20 & 14 & 2 & 21 \\
\hline Ends & & M & 11 & 6 & 19 & 13 & 7 & 16 \\
\hline & & 0 & 7 & 3 & 8 & 14 & 0 & 5 \\
\hline & & $\mathrm{E}$ & 13 & 5 & 3 & 11 & 0 & 6 \\
\hline Bicycle & W 11 - 1 & $\mathrm{Y}$ & 15 & 2 & 17 & 15 & 1 & 21 \\
\hline Crossing & & M & 15 & 2 & 19 & 14 & 5 & 17 \\
\hline & & 0 & 8 & 0 & 10 & 9 & 1 & 9 \\
\hline & & $\mathrm{E}$ & 12 & 1 & 8 & 11 & 1 & 5 \\
\hline No Parking & R 8-3a & $Y$ & 12 & 2 & 21 & 14 & 3 & 20 \\
\hline & & M & 23 & 3 & 10 & 23 & 3 & 10 \\
\hline & & 0 & 13 & 1 & 4 & 13 & 0 & 6 \\
\hline & & $\mathrm{E}$ & 17 & 2 & 2 & 14 & 0 & 3 \\
\hline
\end{tabular}

$\underline{\text { Novel Signs }}$

\begin{tabular}{|c|c|c|c|c|c|c|c|c|}
\hline Sign Name & $\underline{\text { MUTCD\# }}$ & Age & & Male & & & ma & \\
\hline Response** & & & 0 & 1 & 2 & 0 & 1 & 2 \\
\hline Truck & - & $\mathrm{Y}$ & 4 & 1 & 30 & 5 & 3 & 29 \\
\hline Entrance & & M & 1 & 4 & 31 & 4 & 9 & 23 \\
\hline & & 0 & 1 & 0 & 17 & 7 & 1 & 11 \\
\hline & & E & 10 & 1 & 10 & 7 & 0 & 10 \\
\hline School & - & $\mathrm{Y}$ & 13 & 3 & 19 & 6 & 8 & 23 \\
\hline Bus Stop & & M & 3 & 10 & 23 & 5 & 8 & 23 \\
\hline & & 0 & 4 & 5 & 9 & 4 & 10 & 5 \\
\hline & & $\mathrm{E}$ & 6 & 9 & 6 & 6 & 9 & 2 \\
\hline Horse & - & Y & 5 & 12 & 18 & 4 & 14 & 19 \\
\hline Drawn & & M & 5 & 11 & 20 & 5 & 10 & 21 \\
\hline Vehicles & & 0 & 4 & 4 & 10 & 9 & 3 & 7 \\
\hline & & E & 8 & 6 & 7 & 10 & 4 & 3 \\
\hline
\end{tabular}


Table 36. Number of responses to each symbol as a function of age and gender (continued). Novel Signs

\begin{tabular}{lcccccccr} 
Sign Name & MUTCD\# & $\underline{\text { Age }}^{*}$ & \multicolumn{3}{c}{ Males } & \multicolumn{3}{c}{ Females } \\
Reduced & - & Y & 4 & 2 & 29 & 11 & 3 & 23 \\
Legibility & & M & 4 & 3 & 29 & 9 & 7 & 20 \\
& & 0 & 4 & 1 & 13 & 6 & 3 & 10 \\
Crosswinds & & E & 7 & 1 & 13 & 7 & 5 & 5 \\
& & Y & 5 & 1 & 29 & 5 & 2 & 30 \\
& & M & 6 & 0 & 30 & 17 & 3 & 16 \\
& & & 2 & 0 & 16 & 11 & 1 & 7 \\
& & E & 15 & 0 & 6 & 14 & 0 & 3
\end{tabular}

Table 37. Mean percentage correct comprehension of each symbol for new and revised

$\underline{\text { Sign }}$

Modified Signs

Right Curve

No Parking *

Hospital

Cross Road *

Keep Right

Divided

Highway Ends

Lane Reduction *

Transition

Mean

$\underline{\text { Redesigned Signs }}$

Mandatory
Seat Belts
Advance Flagger *
Ranger Station *
Campfire
Pavement Ends *
Bicycle Crossing
No Parking
(Pictograph)

$\underline{\text { Mean }}$ signs as a function of age.

\begin{tabular}{|c|c|c|c|}
\hline \multicolumn{4}{|c|}{ Age Group } \\
\hline$\leq 40$ & 40 to 59 & 60 to 69 & $\underline{70+}$ \\
\hline 93.1 & 97.2 & 91.9 & 92.1 \\
\hline 100.0 & 95.8 & 89.2 & 81.6 \\
\hline 95.8 & 91.7 & 89.2 & 84.2 \\
\hline 90.3 & 94.4 & 11 & 60.5 \\
\hline 88.9 & 90.3 & 89.2 & 60.5 \\
\hline 69.4 & 55.6 & 62.2 & 47.4 \\
\hline 79.2 & 55.6 & 51.4 & 28.9 \\
\hline$\underline{88.1}$ & $\underline{82.9}$ & $\underline{79.2}$ & $\underline{65.1}$ \\
\hline
\end{tabular}

$\underline{40 \text { to } 59} \quad \underline{60 \text { to } 69} \quad \underline{70+}$

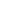


Table 37. Mean percentage correct comprehension of each symbol for new and revised signs as a function of age (continued).

$\underline{\text { Sign }}$

\begin{tabular}{|c|c|c|c|c|}
\hline & $\leq 40$ & 40 to 59 & 60 to 69 & $\underline{70+}$ \\
\hline \multicolumn{5}{|l|}{ Novel Signs } \\
\hline Truck Entrance $*$ & 87.5 & 93.1 & 78.4 & 55.3 \\
\hline School Bus Stop Ahead & 73.6 & 88.9 & 78.4 & 68.5 \\
\hline Horse Drawn Vehicles * & 87.5 & 86.1 & 64.9 & 52.8 \\
\hline Reduced Legibility & 79.2 & 82.0 & 77.0 & 63.2 \\
\hline Cross Winds & 86.1 & 68.1 & 64.9 & 23.7 \\
\hline Mean & $\underline{82.8}$ & $\underline{83.6}$ & $\underline{72.7}$ & $\underline{52.7}$ \\
\hline
\end{tabular}

It is worth briefly examining the more commonly occurring errors. A high proportion of subjects (16.9 percent) had the same wrong response-- "rough road" to the PAVEMENT ENDS symbol. This error was more common among the two older groups, each of which gave this response approximately 26 percent of the time. There was no relationship between this reply and gender or location. The response "rough road" was scored wrong, rather than partly correct, as the road following the end of pavement would not necessarily be rough. There is a separate sign-ROUGH ROAD (W8--8) for this message. The response "slippery road" was given by 9.6 percent of the subjects for the novel CROSS WIND symbol, while 4.6 percent thought the ADVANCE FLAGGER was a school crossing guard.

A series of Chi square tests were used to make comparisons (separately for the four age groups as well as for the two older groups combined, and for all subjects combined) between comprehension of the messages in the two comprehension studies. The findings are shown in table 40. It can be seen that overall improvement resulted for three messages, while comprehension was worse for four. There appears to be no systematic relationship between age and changes in symbol comprehension. If the pictographic version of the NO PARKING message is not included, there is an even split between those symbols which showed an improvement and those which showed a decrement in comprehension.

These comparisons suggest that improvements in understanding can be accomplished through redesign, especially for those symbol designs that are poorly understood. Of those showing significant improvement, two were categorized as poor, and one as intermediate, on the basis of phase 1 results. Those on which performance was worse on the revised symbols were good or intermediate signs in the initial study. This suggests that it may be easier to improve on the understandability of those symbols that are poorly understood by most drivers. 
Table 38. Percentage correct comprehension and mean familiarity ratings for standard and new symbols.

\begin{tabular}{|c|c|c|c|c|c|c|}
\hline & & & & Signs & & \\
\hline$\underline{\text { Rank* }^{*}}$ & Sign Names & & $\underline{\text { Standard }}$ & $\underline{\text { New }}$ & $\begin{array}{r}\text { Mean } \\
\text { Standard }\end{array}$ & arity \\
\hline 1 & Right Curve & & 94.9 & 94.1 & 1.62 & 1.35 \\
\hline 2 & № Parking & c & 95.4 & 93.6 & 1.38 & 1.37 \\
\hline 3 & Hospital & $\mathrm{c}$ & 90.6 & 91.3 & 1.65 & 1.70 \\
\hline 4 & Cross Road & $\mathrm{a}$ & 92.9 & 84.9 & 2.98 & 2.41 \\
\hline 5 & Keep Right & $\mathrm{c}$ & 85.8 & 84.5 & 2.10 & 2.29 \\
\hline 6 & Divided Hwy Ends & $\mathrm{a}$ & 71.7 & 59.8 & 2.08 & 2.19 \\
\hline 7 & Lane Red Trans & $a b$ & 38.1 & 58.0 & 2.14 & 2.97 \\
\hline & $\underline{\text { Mean }}$ & & $\underline{81.4}$ & $\underline{80.9}$ & $\underline{1.99}$ & $\underline{2.03}$ \\
\hline & & & & Signs & & \\
\hline$\underline{\text { Rank* }}$ & $\underline{\text { Sign Names }}$ & & 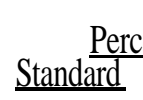 & ${ }^{\mathrm{ct}}$ & $\underline{\text { Standard }}$ & rity \\
\hline 1 & $\begin{array}{l}\text { Mandatory } \\
\text { Seat Belt }\end{array}$ & $a c$ & 74.4 & 85.8 & 3.45 & 3.39 \\
\hline 2 & $\begin{array}{l}\text { Advance } \\
\text { Flagger }\end{array}$ & a & 69.5 & 81.7 & 1.71 & 1.98 \\
\hline 3 & Ranger Station & $b$ & 62.1 & 67.1 & 3.09 & 4.09 \\
\hline 4 & Campfire & $a b c$ & 73.3 & 54.8 & 2.98 & 3.53 \\
\hline 5 & Pavement Ends & & 64.4 & 63.8 & 4.15 & 4.12 \\
\hline 6 & $\begin{array}{l}\text { Bicycle } \\
\text { Crossing }\end{array}$ & $b$ & 44.8 & 68.8 & 2.24 & 3.55 \\
\hline 7 & $\begin{array}{l}\text { No Parking } \\
\text { (Pictograph) }\end{array}$ & a & 95.4 & 31.9 & 1.38 & 3.30 \\
\hline Meal & & & $\underline{69.1}$ & $\underline{64.8}$ & $\underline{2.71}$ & $\underline{3.39}$ \\
\hline & & & & & & \\
\hline Rank* & $\underline{\text { Sign Names }}$ & & & rect & Mean Fam & \\
\hline & Truck Entrance & & & & 3.62 & \\
\hline & $\begin{array}{l}\text { School Bus } \\
\text { Stop Ahead }\end{array}$ & & & & 2.70 & \\
\hline & $\begin{array}{l}\text { Horse Drawn } \\
\text { Vehicles }\end{array}$ & c & & & 4.08 & \\
\hline & Reduced Legibility & & & & 4.59 & \\
\hline & Cross Winds & & & & 4.07 & \\
\hline & Mean & & & & 3.85 & \\
\hline
\end{tabular}


Table 39. Percentage correct comprehension of each sign type as a function of age: comprehension study (phase 2).

$\begin{array}{lccc}\text { Sign Category } & \leq 40 & \frac{\text { Driver Age }}{40 \text { to } 59} & \underline{60+} \\ \text { Modified } & 88.1 & \frac{82.9}{72.1} & 47.9 \\ \text { Redesigned } & 76.9 & 68.9 & 62.7 \\ \text { Novel } & 82.8 & 83.6 & \end{array}$

Table 40. Percentage difference in mean comprehension between standard and new signs.

\section{Percentage Difference by Age Group}

\begin{tabular}{lcccccc} 
Modified Signs & 18 to 39 & $\underline{40 \text { to } 59}$ & $\underline{60 \text { to } 69}$ & $\underline{70+}$ & $\underline{60+}$ & $\underline{\text { All }}$ \\
\cline { 2 - 5 } & -3.9 & -0.3 & +0.7 & +5.0 & +2.3 & -0.7 \\
No Parking & +2.6 & -1.7 & -4.4 & -5.6 & -6.1 & -1.9 \\
Hospital & +0.1 & -0.2 & +6.4 & -0.7 & +2.8 & +0.8 \\
Cross Road & -6.8 & -0.6 & -4.6 & -25.3 & -14.7 & $-7.8^{*}$ \\
Keep Right & +0.1 & +1.7 & +9.7 & -19.0 & -4.0 & -0.8 \\
Lane Reduction & & & & & & \\
Transition & $+65.7^{*}$ & +50.7 & +52.1 & +14.2 & +35.9 & $+52.2^{*}$ \\
Divided Highway Ends & -6.1 & -25.9 & +3.7 & -26.6 & -12.0 & -15.1
\end{tabular}

Percentage Difference by Age Group

\begin{tabular}{|c|c|c|c|c|c|c|}
\hline Redesigned Signs & 18 to 39 & $\underline{40 \text { to } 59}$ & $\underline{60 \text { to } 69}$ & $\underline{70+}$ & $\underline{60+}$ & $\underline{\text { All }}$ \\
\hline Mandatory Seatbelts & +2.5 & +13.6 & +50.7 & +41.1 & +46.7 & +15.3 \\
\hline Advance Flagger & +21.3 & +12.0 & +42.5 & +4.0 & +23.3 & +18.1 \\
\hline Ranger Station & +29.7 & +0.4 & -12.6 & -5.0 & -9.3 & +9.6 \\
\hline Campfire & -8.1 & +7.3 & -53.7 & -49.0 & -51.5 & -16.5 \\
\hline Bicycle Crossing & +22.1 & +27.3 & +23.5 & +3.9 & +14.4 & +21.2 \\
\hline $\begin{array}{l}\text { No Parking } \\
\text { (pictogram) }\end{array}$ & -34.4 & -63.0 & -57.4 & -79.2 & -68.0 & -56.9 \\
\hline Pavement Ends & -24.9 & +2.6 & +13.6 & +3.9 & -6.3 & -12.1 \\
\hline
\end{tabular}

Note:

Positive difference indicates greater comprehension of new sign.

* $\mathrm{p}<.01$ 


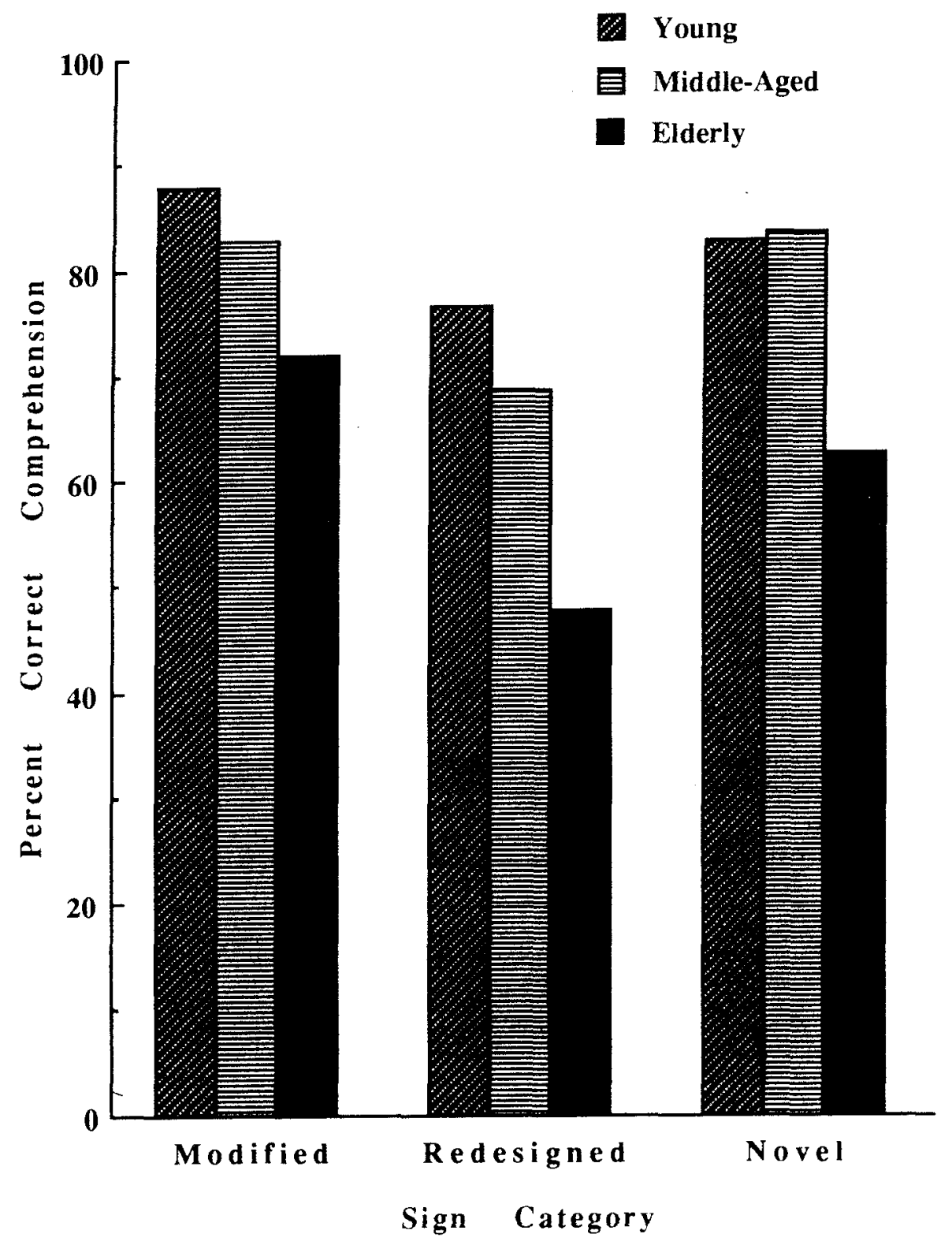

Figure 20. Mean percent correct comprehension of Modified, Redesigned, and Novel signs as a function of driver age. 


\section{Familiarity Of Novel Symbols}

The five completely new symbols were rated as quite unfamiliar $(1=$ very familiar; $5=$ very unfamiliar), with the exception of SCHOOL BUS STOP AHEAD (mean = 2.70), and TRUCK ENTRANCE (mean = 3.62). The relatively high familiarity of these two may be due to location differences. In Alberta, the SCHOOL BUS STOP AHEAD symbol in use is similar to that in this study, and a Canadian TRUCK ENTRANCE symbol in widespread use has some similarities to the one tested here. Comparison of the mean familiarity ratings of these signs across the three locations indicated that the Alberta sample did rate greater familiarity for both the school bus (Alberta $=2.17$; Idaho $=3.19$; Texas $=2.74)$ and the truck (Alberta $=3.30$; Idaho $=3.88$; Texas $=3.69$ ) signs. The only other regional difference was that the Texas sample indicated slightly greater familiarity with the HORSE DRAWN VEHICLE symbol. Both older groups rated the school bus symbol as more familiar, while the 60 to 69 age group rated the horse drawn vehicle and the truck entrance symbols as more familiar than did the two younger groups.

Statistically significant correlations $(\mathrm{p}<.01)$ between comprehension and ratings of familiarity were found for 32 percent of the symbols (see table 39). This is less than half the 67 percent found in the initial comprehension study, due in part possibly to smaller numbers of subjects tested in the present study. As before, more familiar symbols were better understood.

\section{CONCLUSIONS}

Redesign of existing symbols resulted in increased comprehension for three messages, while modification did little to improve symbol comprehension. The greater effect of redesign (which involved either a complete or a major change in sign content) was expected, since the intent of redesign was to improve comprehension and the intent of modification was to enhance legibility. The relatively good understanding of the novel symbols (equal to that of the modified ones) suggested that the technique employed here to create easily understood new symbols, was an effective one. 



\section{CHAPTER 11: DAYTIME LEGIBILITY OF MODIFIED, REDESIGNED, AND NOVEL HIGHWAY SIGNS}

Failure to heed signs is one of the leading causes of automobile accidents among older drivers and not surprisingly, they are more likely than young drivers to report difficulty in reading signs in time to respond to them. ${ }^{(5,10)}$ Research has also shown that, compared to their younger counterparts, the legibility distance of highway signs is reduced among older drivers. ${ }^{(30)}$ The purpose of this study was to evaluate the degree to which the legibility distance of symbol signs could be enhanced among drivers of different age. Specifically, this investigation sought to evaluate the daytime legibility and comprehension of a series of modified, redesigned and novel symbol highway signs among young, middle-aged, and older drivers, and determine the extent to which differences in either measure are related to static visual acuity and/or contrast sensitivity.

Kline and Fuchs evaluated the degree to which the legibility distance advantages of symbol signs could be further increased by using a low-pass optical filtering technique to optimize sign contour size and separation. ${ }^{(57)}$ To identify the details of each sign that were most difficult to resolve, or distinguish from their adjacent features, they were reiteratively viewed and redesigned through blur induced by strong positive (5 to $7 \mathrm{D})$ spherical lenses. The mean legibility distance of the symbol signs that resulted from this process was about three times that of the standard word versions and about 50 percent greater than that of standard symbol signs for young, middle-aged, and older drivers. The investigators attributed this improvement to reductions in the degree to which the signs depended on high spatial frequency information to convey their message. Consistent with this explanation, acuity, a strong predictor of the legibility distance of standard word and symbol signs, was only weakly related to the legibility of the redesigned signs.

Although the low-pass optical filtering technique appears to be an effective tool for enhancing the legibility of symbol signs, it does not provide precise control over spatial frequency characteristics of the display. By allowing the researcher to determine the form and range of spatial filters, contemporary image-processing techniques, however, can be utilized to overcome this limitation. Image-processing techniques can also be used to relate the visual abilities of individual observers to the spatial characteristics of specific displays, and thus provide a direct quantitative index of display legibility without the need for driver testing. For these reasons, the present study used to a computer-based image-processing approach to modify and redesign standard symbol signs, as well as to design new signs.

\section{METHOD}

\section{Subjects}

Three age groups of active, licensed, community-resident drivers participated in the study: a young group (6 men and 6 women), $\underline{M}$ age $=25.4$ years (range 20 to 31 ), a middle-aged group ( 6 men and 6 women), $\underline{\mathrm{M}}$ age $=52.1$ (range 38 to 59), and an older group (9 men and 9 women), $\underline{M}$ age $=69.0$ (range 60 to 84 ). More older drivers were included due to the greater variability performance typically observed both within and between older individuals. The mean number of years of formal education was 14.3 years (range 12 to 17) for the young, 15.7 years (range 12 to 22) for the middle-aged, and 13.9 years (range 9 to 20) for the older. None of the age differences in education were significant $(\mathrm{p}=0.32$ ). Information regarding the 
participants' driving experience is presented in table 41. As can be seen there, older persons reported driving less at night or on highways than young and middle-aged drivers. Consistent with their report that their driving was done principally in a suburban setting, older drivers were less likely to drive a very high or very low number of kilometers a year. Like young drivers, the older drivers were less likely than the middle-aged to drive during rush hours. Compared to their male cohorts, women, especially older ones, report having fewer years of driving experience. Other than a higher level of motorcycle driving among young drivers, there were few age differences in type of vehicle driven.

Overall, the participants were in good general health for their age group. Older participants ( 6 of 18) were more likely than young ( 1 of 12), or middle-aged (I of 12) drivers to have a chronic illness or complaint. The older participants ( 8 of 18) were also more likely than the young ( 2 of 12$)$ or the middle-aged ( 2 of 12$)$ to take one or more medications on a chronic basis.

The visual health of the participants was generally good, although as might be expected, reports of visual problems increased with age. One young, one middle-aged, and two older drivers reported being color blind, and one middle-aged and one older driver were blind in one eye. Other visual problems reported by older drivers were: "lazy eye" (1), cataracts (3), glaucoma (1) and inter-ocular implant (1). Mean presenting binocular photopic Landolt $\mathrm{C}$ acuity levels were 0.63 minarc (range .50 to 1.12 ) for the young, 0.68 minarc (range .50 to 1.32) for the middle-aged, and 0.81 minarc (range .50 to 1.46 ) for the older participants. Spherical optimization of presenting acuity resulted in excellent best acuity levels for all three age groups: 0.58 minarc (range .50 to 0.88 ) for the young, 0.62 minarc (range .50 to 1.12 ) for the middleaged, and 0.74 minarc (range .50 to 1.46$)$ for the older participants $(1.0$ minarc $=20 / 20)$. The acuity levels of the older drivers was better than the average for their age group, a point confirmed by a one-way ANOVA showed that acuity levels of the young, middle-aged, and older drivers were not significantly different from each other. For most participants: young (8 of 12), middle-aged, (10 of 12), and older (14 of 18), no additional correction was needed to optimize acuity. Of those who required the additional refraction, only 3 (1 young, 2 older) needed additional correction for myopia (range -0.25 to $-0.50 \mathrm{D}$ ), while 7 (3 young, 2 middle-aged, and 2 older) needed additional correction for hyperopia (range +0.25 to $+0.50 \mathrm{D}$ ).

\section{Stimuli And Apparatus}

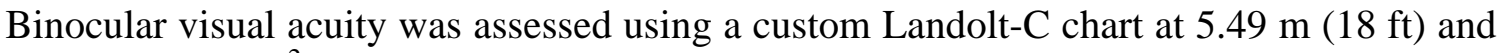
a luminance of $77 \mathrm{~cd} / \mathrm{m}^{2}$. Following the establishment of participants' presenting level, acuity was optimized refractively using a Bausch and Lomb Master Phoropter. Contrast sensitivity was then measured at 5 spatial frequencies: 1.25, 2.5, 5.0, 10.0, and 22.5 cycles per degree, using a CRT-based analog of the Vistech VCTS 6500 far chart at a viewing distance of $6.1 \mathrm{~m}(20 \mathrm{ft})$ at a space-averaged luminance of $22 \mathrm{~cd} / \mathrm{m}^{2}$. The luminance of surfaces in the vicinity of the monitor was-also maintained at approximately $22 \mathrm{~cd} / \mathrm{m}^{2}$. 
Table 41. Participant demographics: daytime legibility of Modified, Redesigned, and Novel symbol signs

Average number of years driving experience as a function of age and sex

\begin{tabular}{lccc}
\hline Sex & Young & $\frac{\text { Age Group }}{\text { Middle-Aged }}$ & Older \\
Males & 9.7 & $\frac{36.3}{52.8}$ & 39.0 \\
Females & 6.0 & 28.7 & 45.9 \\
Means & 78 & 32.5 &
\end{tabular}

Average number of years held a driver's license

Age Group

$\frac{\text { Sex }}{\text { Males }} \quad \frac{\text { Young }}{8.7} \quad \frac{\text { Middle-Aged }}{35.8} \quad \frac{\text { Older }}{52.0}$

$\begin{array}{llll}\text { Females } & 5.5 & 28.8 & 38.7\end{array}$

$\begin{array}{llll}\text { Means } & 7.1 & 32.3 & 45.3\end{array}$

Percentage of drivers by distance driven last 12 months as a functionof age

\begin{tabular}{|c|c|c|c|}
\hline Distance Driven & & Age Group & \\
\hline (miles) & $\underline{\text { Young }}$ & Middle-Aged & $\underline{\text { Older }}$ \\
\hline under 5,000 & 33.3 & 33.3 & 11.1 \\
\hline $5,000-9,999$ & 33.3 & 8.3 & 55.6 \\
\hline $10,000-14,999$ & 16.8 & 25.0 & 16.7 \\
\hline $15,000-19,999$ & 8.3 & 16.7 & 11.1 \\
\hline 20,000 or more & 8.3 & 16.7 & 5.5 \\
\hline
\end{tabular}

Percentage of drivers by driving environment as a function of age

Age Group

$\begin{array}{lccc}\text { Driving Environment } & \text { Young } & \text { Middle-Aged } & \text { Older } \\ \text { Rural/sparsely populated } & - & - & - \\ \text { Small town } & 8.3 & - & - \\ \text { Suburban } & 25.0 & 50.0 & 66.7 \\ \text { High-density urban } & 41.6 & 41.7 & 22.2 \\ \text { Highway } & 25.0 & 8.3 & 11.1\end{array}$

Percentage of drivers by night driving as a function of age

\begin{tabular}{lccc} 
Percentage Night & \multicolumn{3}{c}{ Age Group } \\
Driving & Young & $\frac{1}{\text { Middle-Aged }}$ & Older \\
\hline $0-5$ & 16.7 & 8.3 & 16.7 \\
$6-10$ & - & 41.7 & 33.3 \\
$11-15$ & 16.7 & 8.3 & 11.1 \\
$16-20$ & 16.7 & 25.0 & 27.7 \\
$21-30$ & 33.3 & 16.7 & 56 \\
$31-40$ & 8.3 & - & 5.6 \\
$41-50$ & 8.3 & - & - \\
51 or more & - & - & -
\end{tabular}


Table 41. Participant demographics: daytime legibility of Modified, Redesigned, and Novel symbol signs (continued).

Percentage of drivers by driving done during rush hour as a function of age

\section{Percentage}

$\underline{\text { Rush Hour }}$

$0-5$

$6-10$

$11-15$

$16-20$

$21-30$

$31-40$

$41-50$

51 or more
Age Group

$\underline{\text { Middle-Aged }}$

$\underline{\text { Older }}$

$\frac{\text { Young }}{25.0}$

8.3

16.7

16.7$$
167
$$

16.7

16.7

$\begin{array}{cc}- & 33.3 \\ 50.1 & 44.4 \\ 8.3 & 5.6 \\ - & - \\ 8.3 & 11.1 \\ - & 5.6 \\ - & - \\ 33.3 & -\end{array}$

Percentage of Drivers by driving done on the highway as a function of age

\section{Percentage}

Highway

$0-5$

$6-10$

$11-15$

$16-20$

$21-30$

$31-40$

$41-50$

51 or more

\section{Age Group}

\section{Middle-Aged}

16.7

8.3

8.3

25.0

16.7

8.3

8.3

$-$

8.3
$-$

8.3

16.7
16.7

$\underline{\text { Older }}$

16.6

44.4

11.1

5.6

5.6

Percentage of drivers by type of vehicle driven as a function of age

\section{Age Group}

Type of Vehicle

Passenger Car

Motor home/W

Motorcycle

Light Truck/Van

Heavy Truck

Bus
Middle-Aged

91.7

91.7

8.3

$-$ $\underline{\text { Older }}$

88.9

8.3

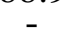

11.1

Legibility distance and comprehension levels were determined for 19 symbol highway signs (appendix H). Of these, seven were "Modified" and seven were "Redesigned" versions of signs from the MUTCD; another five were "Novel" signs. ${ }^{(3)}$ Modified signs (figure 16) employed the basic spatial layout of the Manual version to maintain their comprehensibility. Redesigned signs (figure 18) employed a wholly new spatial layout to convey the same message as a corresponding version in the Manual, and Novel signs (figure 19) were newly designed signs that presented a message not in the Manual. The design approach used to develop these three sign types is described in chapter 9 (see figure 16). Signs were presented in their regulation colors on a white background using an Apple High-Resolution 330-mm (13-in) color monitor, 
driven by a Raster Ops 364 24-bit video board and controlled by a Macintosh IIci computer. The luminance of the white background was maintained at $80 \mathrm{~cd} / \mathrm{m}^{2}$. Participants viewed the signs through the Phoropter from $5.5 \mathrm{~m}(18 \mathrm{ft})$. Luminance levels of the acuity and contrast sensitivity tests were maintained using a Minolta LS- 110 spot photometer; screen luminance of the monitor was calibrated with a UDT model 61 -CRT photometer.

\section{Procedure}

\section{$\underline{\text { Sign Legibility Threshold }}$}

Following acuity and contrast sensitivity testing, participants were adapted to the light level in the test setting for approximately $5 \mathrm{~min}$ as the sign legibility and comprehension task was explained to them. To simulate increases in a sign's visual angular size as it is approached by a driver, it was initially presented on the monitor screen at a size too small to be legible. From there, its size was increased in seven percent steps. At each step, observers were requested to describe all of the sign's features that they could. The smallest size at which all of a sign's critical features could be described, according to a pre-determined scoring key (see appendix J: Day Legibility Scoring Key), was recorded as its threshold. The scoring key was based on an a priori identification by the experimenters of the basic details in each sign that distinguished it from other signs, and which were deemed necessary to convey its message completely. For example, the PAVEMENT ENDS sign was increased in size until observers were able to identify the upward arrowhead, the car heading right, the straight pavement line below the car on the left, and the broken undulating line below the car on the right. To assume that a subject's response was as complete as possible, their identification was cued, first on a location basis and then, if needed, by relational prompts. Location cues consisted of asking the observer if they could identify any features in a particular quadrant (e.g., "lower left") of the sign. If needed, followup relational prompts asked observers to identify a feature or features by referring to its position in relation to a feature the observer had already described (e.g., "is there anything to the left of the arrow?").

Once observers had correctly identified all the critical features of a sign, a full-screen version of the sign was displayed, and observers were asked to state its intended message. Comprehension was scored as correct (2), partially correct (1), or incorrect (0) for each sign according to a pre-determined scoring criterion (see appendix I). The comprehension score for each observer was recorded as percentage of the total possible score out of 38 (i.e., 19 signs $\mathrm{x}$ a maximum score of 2).

The 19 signs were presented in one of six different random orders. In the young and middle-aged groups, one male and one female participant each received one of the orders. Among the older, where each age by sex group was composed of three extra participants, three of the six orders were repeated among the men and three orders were repeated among the women. To avoid cueing effects associated with the second presentation of a NO PARKING Sign, the pictographic car version was given last in all presentation orders. The presentation rate of the signs was adjusted to the comfort level of each participant. Testing occurred in a single session lasting approximately one and a half hours. Participants received an honorarium of $\$ 10 / \mathrm{h}$ of time in the study. 


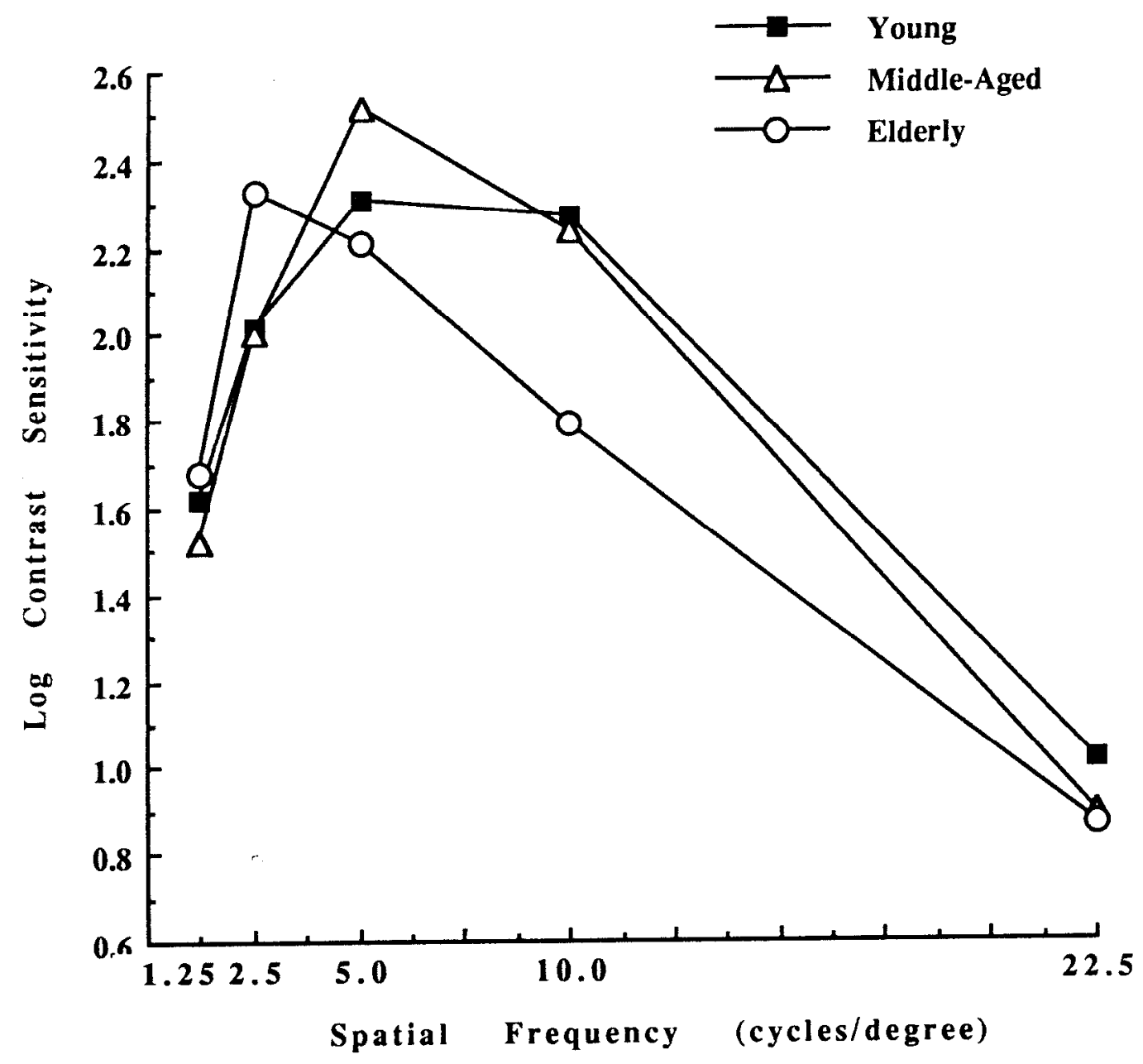

Figure 21. Log contrast sensitivity functions for young, middle-aged, and elderly drivers: daytime legibility (phase 2). 


\section{Low Cut-Off Filter Thresholds}

To determine the general relationship between a sign's legibility threshold and its resistance to low-pass filtration, the lowest filter cut-off level at which each sign's critical details was preserved was determined for two additional young observers. Each sign was filtered at successively lower cut-off levels until the first level at which one or more of its critical details could not be discerned was reached. The lowest level at which the observer could still discern all the details in the legibility scoring key was recorded as the low-pass threshold for that sign.

\section{RESULTS}

\section{Contrast Sensitivity}

An Age (3) x Spatial Frequency (5) mixed design ANOVA carried out on the log contrast sensitivity data revealed significant effects for both spatial frequency $[\mathrm{F}(4,156)=137.41$, $\mathrm{p}<0.001]$, and the age by spatial frequency interaction $[\mathrm{F}(8,156)=2.12, \mathrm{p}<0.051$. The main effect for age was not significant. Dependent T-test comparisons (with the Bonferroni correction) collapsed across age indicated significant differences $(\mathrm{p}<0.05$ or better) on all possible pair-wise comparisons of the five spatial frequencies. Tukey HSD pair-wise comparisons used to examine the age by spatial frequency interaction indicated that the age difference only approached significance $(p=0.068)$ for young and older drivers at $22.5 \mathrm{c} / \mathrm{deg}$ (See figure 21).

To determine the utility of a single CSF measure for predicting the legibility distance of symbol signs, several composite measures were extracted from each individual's CSF: peak sensitivity, peak spatial frequency, low cutoff, high cutoff, and bandwidth (i.e., the difference between the high and low cutoff measures). For four observers (two young, one middle-aged, and one older), the contrast sensitivity function did not fall below half of its peak value at low spatial frequencies, so it was not possible to derive either the low cutoff or bandwidth measure. No significant age differences were seen on the log of the mean peak sensitivity, peak spatial frequency, or the high cutoff measures (see table 42).

Table 42. Age differences in derived contrast sensitivity measures.

\begin{tabular}{|c|c|c|c|c|}
\hline & Young & $\begin{array}{l}\text { Middle- } \\
\underline{\text { Aged }}\end{array}$ & $\underline{\text { Older }}$ & $\underline{\text { Age Diff }}$ \\
\hline Log of Mean Peak Sensitivity & 2.488 & 2.553 & 2.382 & none \\
\hline Peak Spatial Frequency (c/deg) & 5.833 & 6.667 & 4.722 & none \\
\hline High Cutoff (c/deg) & 9.541 & 9.958 & 8.359 & none \\
\hline
\end{tabular}

\section{Sign Legibility Distance}

To estimate the on-the-road legibility of each sign, its size threshold was converted to legibility distance based on their regulation size: Legibility distance $(\mathrm{m})=($ Regulation Sign Size $(\mathrm{m}) /$ Thresholder Size $(\mathrm{m}))$ x Test Distance $(5.5 \mathrm{~m})$. Since the effect of gender was not significant in the initial omnibus Age (3) by Gender (2) by Sign Type (3) ANOVA, an Age (3) by Sign Type (3) ANOVA was carried out. Significant differences were observed for Age [F $(2,39)$ $=7.56, \mathrm{p}<0.01]$, Sign Type $[\mathrm{F}(2,78)=741.3, \mathrm{p}<0.01]$, and the Age by Sign Type interaction 
$[\mathrm{F}(4,78)=4.11, \mathrm{p}<0.01]$. As can be seen in both table 43 and figure 22 , the mean legibility distance of Modified signs greatly exceeded that of Novel $(\mathrm{p}<.01)$ and Redesigned signs, the legibility of Novel signs also exceeded that of Redesigned $(\mathrm{p}<.05)$. Mean legibility distances were greatest among young drivers, lowest among the older driver, and intermediate for the middle-aged, particularly for Modified signs. One-way ANOVA's revealed significant age differences for Modified $[\mathrm{F}(2,39)=6.55, \mathrm{p}<0.01]$, Redesigned $[\mathrm{F}(2,39)=5.34, \mathrm{p}<0.01]$, and Novel signs

$[\mathrm{F}(2,39)=7.65, \mathrm{p}<0.01]$. Although age differences were greatest for Modified signs, Tukey pair-wise comparisons indicated that mean sign legibility distances were greater for younger drivers than older ones for all three sign types $(\mathrm{p}<0.01)$. Legibility distances of middle-aged drivers were not significantly different from their younger or older counterparts for any sign type, although the middle-aged/older difference approached significance for Novel signs $(\mathrm{p}=.079)$.

Tukey HSD comparisons of the age differences on individual signs indicated that the legibility distances of younger drivers. significantly exceeded those of older drivers on 12 of the 19 signs (four signs in each of the three types). The legibility distance of middle-aged drivers, although not significantly different from the young for any sign, exceeded that of the older driver on only one sign (CROSS WIND).

To determine the degree to which their legibility had been changed, the overall legibility distance of Modified and Redesigned signs was compared with that assessed in the previous study of the daytime legibility of standard signs (see table 44). The legibility of Modified signs exceeded that of the Standard version for six of the seven signs, a difference that was significant on four of them. The degree of improvement ranged from $149 \mathrm{~m}$ (43 percent) to $2.1 \mathrm{~m}$ (1.6 percent); the average improvement was $52.1 \mathrm{~m}$ (22 percent). On the seventh sign (LANE REDUCTION TRANSITION) legibility was significantly reduced (81.7 m or 37.3 percent). Among Redesigned signs, legibility was increased for five signs (three significantly) and reduced for two (both significantly). The average improvement was $12.3 \mathrm{~m}$ (11.2 percent), and the average reduction was $47.5 \mathrm{~m}$ (34 percent).

An Age (3) by Study (2) by Sign Type (3) ANOVA yielded significant main effects for Age $[F(2,78)=10.76, p<0.01]$, Sign Type $[F(1,78)=1326.81, p<0.01]$, Sign Type by Age $[\mathrm{F}(2,78)=5.44,<0.01]$, and Sign Type by Study $[\mathrm{F}(1,78)=24.95, \mathrm{p}<0.01]$ interactions. The overall difference between Modified/ Redesigned versus Standard signs approached but did not reach significance $(\mathrm{p}=0.067)$. The mean legibility distance of signs in the Modified category exceeded that of Redesigned signs $(\mathrm{p}<.01)$, and so too did their degree of improvement over the Standard version. Associated with their greater overall legibility distance, the age deficit in legibility was also somewhat more prominent for signs in the Modified category. To determine the degree to which modification or redesign of individual signs affected the legibility of individual signs for young, middle-aged, and older drivers, the differences between the New and Standard versions on legibility distance were compared separately for each age group (tables 45, 46, and 47). The general pattern of differences between the New and Standard individual signs within each age group was similar to the overall pattern. Only among the older for Modified signs (see table 47), however, was the net difference from standard signs significant $(\mathrm{p}<0.05)$. Younger and older drivers appeared to benefit more than the middle-aged from the modification or redesign of individual signs. The legibility distance of 10 signs (six Modified, four 
Redesigned) was increased for young drivers, and of 11 signs (six Modified, five Redesigned) for older ones. 
Table 43. Mean legibility distance (m) and standard deviations (SD) of 19 Modified, Redesigned, and Novel symbol signs for young, middle-aged, and elderly drivers (continued).

\begin{tabular}{|c|c|c|c|c|c|c|}
\hline \multicolumn{7}{|c|}{$\underline{\text { Redesigned Signs }}$} \\
\hline $\begin{array}{l}\text { Rank } \\
\text { (Dist) } \\
\end{array}$ & $\begin{array}{l}\text { Sign Name } \\
\text { (MUTCD No.) }\end{array}$ & $\begin{array}{l}\text { Age } \\
\text { Diff }\end{array}$ & $\frac{\text { Young }}{(\mathrm{SD})}$ & $\begin{array}{l}\text { Middle- } \\
\text { Aged } \\
\underline{(\mathrm{SD})}\end{array}$ & $\frac{\text { Elderly }}{\underline{(\mathrm{SD})}}$ & $\begin{array}{c}\text { Mean } \\
\text { Dist }\end{array}$ \\
\hline 6. & $\begin{array}{l}\text { Advance Flagger } \\
\text { (W20-7a) }\end{array}$ & - & $\begin{array}{l}216.7 \\
(65.0)\end{array}$ & $\begin{array}{l}198.0 \\
(57.5)\end{array}$ & $\begin{array}{l}191.9 \\
(53.5)\end{array}$ & 202.2 \\
\hline 13. & $\begin{array}{l}\text { Pavement Ends } \\
(\mathrm{W} 8-3 \mathrm{a})\end{array}$ & - & $\begin{array}{l}124.6 \\
(24.4)\end{array}$ & $\begin{array}{l}110.2 \\
(26.4)\end{array}$ & $\begin{array}{l}107.0 \\
(25.7)\end{array}$ & 113.9 \\
\hline 14. & $\begin{array}{l}\text { Bicycle Crossing } \\
\text { (W11-1) }\end{array}$ & $\mathrm{a}$ & $\begin{array}{l}120.9 \\
(24.8)\end{array}$ & $\begin{array}{l}113.5 \\
(24.1)\end{array}$ & $\begin{array}{c}97.1 \\
(21.2)\end{array}$ & 110.8 \\
\hline 15 . & $\begin{array}{l}\text { Campfire } \\
(\mathrm{RA}-030)\end{array}$ & $\mathrm{a}$ & $\begin{array}{l}132.1 \\
(40.6)\end{array}$ & $\begin{array}{r}96.9 \\
(15.3)\end{array}$ & $\begin{array}{r}98.6 \\
(18.1)\end{array}$ & 109.2 \\
\hline 17. & $\begin{array}{l}\text { Ranger Station } \\
(\mathrm{RG}-170)\end{array}$ & a & $\begin{array}{l}104.9 \\
(22.6)\end{array}$ & $\begin{array}{r}90.3 \\
(23.2)\end{array}$ & $\begin{array}{l}72.7 \\
(16.1)\end{array}$ & 89.3 \\
\hline 18. & $\begin{array}{l}\text { Mandatory Seatbelt } \\
(\mathrm{R} 16-1)\end{array}$ & $\mathrm{a}$ & $\begin{array}{c}88.1 \\
(16.9)\end{array}$ & $\begin{array}{r}73.4 \\
(22.4)\end{array}$ & $\begin{array}{l}67.3 \\
(21.0)\end{array}$ & 76.3 \\
\hline 19. & No Parking (Car) & - & $\begin{array}{r}79.7 \\
(19.0)\end{array}$ & $\begin{array}{c}76.8 \\
(16.8)\end{array}$ & $\begin{array}{l}67.2 \\
(23.8)\end{array}$ & 74.6 \\
\hline & Mean & a & 124.0 & 108.4 & 100.3 & 110.9 \\
\hline \multicolumn{7}{|c|}{$\underline{\text { Novel Signs }}$} \\
\hline $\begin{array}{l}\text { Rank } \\
\text { (Dist) } \\
\end{array}$ & $\frac{\text { Sign Name }}{(\mathrm{MUTCD} \text { No, })}$ & $\begin{array}{l}\text { Age } \\
\text { Diff }\end{array}$ & $\frac{\text { Young }}{\underline{\text { (SD) }}}$ & $\begin{array}{l}\text { Middle- } \\
\underline{\text { Aged }} \\
\underline{(\mathrm{SD})}\end{array}$ & $\frac{\text { Elderly }}{(\mathrm{SD})}$ & $\begin{array}{l}\text { Mean } \\
\text { Dist }\end{array}$ \\
\hline 9. & Cross Wind & $\mathrm{ac}$ & $\begin{array}{l}133.2 \\
(16.5)\end{array}$ & $\begin{array}{l}133.4 \\
(27.2)\end{array}$ & $\begin{array}{l}113.8 \\
(18.5)\end{array}$ & 126.8 \\
\hline 10. & Horse Drawn Vehicle & a & $\begin{array}{l}137.6 \\
(26.8)\end{array}$ & $\begin{array}{l}127.5 \\
(23.3)\end{array}$ & $\begin{array}{l}108.0 \\
(21.1)\end{array}$ & 124.4 \\
\hline 11. & Reduced Legibility & $\cdot$ & $\begin{array}{l}133.2 \\
(27.1)\end{array}$ & $\begin{array}{l}120.5 \\
(36.7)\end{array}$ & $\begin{array}{l}109.8 \\
(23.8)\end{array}$ & 121.2 \\
\hline 12. & Truck Entrance & $\mathrm{a}$ & $\begin{array}{l}128.9 \\
(29.5)\end{array}$ & $\begin{array}{l}120.4 \\
(45.4)\end{array}$ & $\begin{array}{c}96.1 \\
(26.0)\end{array}$ & 115.1 \\
\hline \multirow[t]{2}{*}{16.} & $\begin{array}{l}\text { School Bus Stop } \\
\text { Ahead }\end{array}$ & a & $\begin{array}{l}107.9 \\
(22.9)\end{array}$ & $\begin{array}{r}84.8 \\
(15.8)\end{array}$ & $\begin{array}{r}83.5 \\
(17.0)\end{array}$ & 92.1 \\
\hline & Mean & $\mathrm{a}$ & 128.2 & 117.3 & 102.3 & 115.9 \\
\hline
\end{tabular}


Among middle-aged drivers, an improvement in legibility was seen for only six signs (four Modified, two Redesigned).

\section{Visual Predictors Of Sign Legibility}

The utility of visual acuity as a predictor of sign legibility distance (both overall and separately for each age group) was evaluated using Pearson product-moment correlations. (Probability levels were adjusted using a Bonferroni correction.) A strong positive correlation was observed between acuity and sign legibility $(\underline{r}=0.65, \underline{p}<0.001)$ (see table 48). The strength of this relationship varied little with sign type (Modified: $\underline{r}=0.65, p<0.01$; Redesigned $\underline{r}=$ $0.57, \mathrm{p}<0.01$; Novel $\underline{\mathrm{r}}=0.67, \mathrm{p}<0.01$ ). Acuity was significantly related to legibility distance for middle-aged drivers $(\underline{r}=0.77, \underline{p}<0.01)$ and older drivers $(\underline{r}=0.52, p<0.05)$, but only approached significance for the young ones $(r=0.51, p<0.10)$. The high cut-off spatial frequency was not significantly related to legibility distance for any of the three age groups, nor were the high cutoff measures significantly correlated with acuity. The overall correlation between peak log contrast sensitivity and legibility distance was significant $(\underline{r}=0.32, \underline{p}<0.05)$, but the corresponding within-group correlations, were significant for older $(\underline{r}=.48, p<0.05)$, but not young $(\underline{r}=0.02, \underline{p}=0.96)$, or middle-aged drivers $(\underline{r}=0.38, \underline{p}=0.22)$. Peak spatial frequency was not significantly correlated with legibility distance for any of the age groups.

Table 43. Mean legibility distance (m) and standard deviations (SD) of 19 Modified, Redesigned, and Novel symbol signs for young, middle-aged, and older drivers.

\section{$\underline{\text { Modified Signs }}$}

\begin{tabular}{|c|c|c|c|c|}
\hline Age & Young & $\begin{array}{l}\text { Middle- } \\
\text { Aged }\end{array}$ & $\underline{\text { Older }}$ & Mean \\
\hline Diff & $(\mathrm{SD})$ & $(\mathrm{SD})$ & $(\mathrm{SD})$ & $\underline{\text { Dist }}$ \\
\hline- & $\begin{array}{c}548.3 \\
(108.8)\end{array}$ & $\begin{array}{r}473.2 \\
(158.3)\end{array}$ & $\begin{array}{r}467.6 \\
(124.2)\end{array}$ & 496.4 \\
\hline $\mathrm{a}$ & $\begin{array}{l}387.9 \\
(91.4)\end{array}$ & $\begin{array}{c}334.1 \\
(120.7)\end{array}$ & $\begin{array}{l}291.4 \\
(56.6)\end{array}$ & 337.8 \\
\hline- & $\begin{array}{l}307.1 \\
(45.0)\end{array}$ & $\begin{array}{l}294.1 \\
(71.9)\end{array}$ & $\begin{array}{l}265.3 \\
(41.8)\end{array}$ & 288.8 \\
\hline $\mathrm{a}$ & $\begin{array}{l}286.0 \\
(61.1)\end{array}$ & $\begin{array}{l}259.3 \\
(81.9)\end{array}$ & $\begin{array}{l}205.9 \\
(45.1)\end{array}$ & 250.4 \\
\hline $\mathrm{a}$ & $\begin{array}{l}269.0 \\
(48.4)\end{array}$ & $\begin{array}{l}209.5 \\
(45.2)\end{array}$ & $\begin{array}{l}202.0 \\
(60.4)\end{array}$ & 226.8 \\
\hline $\mathrm{a}$ & $\begin{array}{l}156.2 \\
(36.0)\end{array}$ & $\begin{array}{l}137.0 \\
(34.0)\end{array}$ & $\begin{array}{l}118.5 \\
(18.8)\end{array}$ & 137.2 \\
\hline- & $\begin{array}{l}155.4 \\
(39.9)\end{array}$ & $\begin{array}{l}132.5 \\
(37.9)\end{array}$ & $\begin{array}{l}120.4 \\
(44.8)\end{array}$ & 136.1 \\
\hline $\mathrm{a}$ & 301.4 & 262.8 & 238.7 & 267.6 \\
\hline
\end{tabular}

Rank Sign Name

$\underline{\text { (Dist) }}$ (MUTCD No.)

1. Cross Road

(W2 - 1)

2. Right Curve

(W 1-2R)

3. Hospital

(D9-2)

4. Divided Highway

Ends

W 6 - 2)

5. Keep Right

( $\mathrm{RJ}$ - 7)

7. Lane Reduction

Transition

8. No Parking

(R8-3a)

Mean
Notes:

Middle-

$\mathrm{a}=$ Significant difference $(\mathrm{p}<.05)$ between Young and Older Drivers

$\mathrm{c}=$ Significant difference $(\mathrm{p}<.05)$ between Middle-Aged and Older Drivers 
Table 43. Mean legibility distance (m) and standard deviations (SD) of 19 Modified, Redesigned, and Novel symbol signs for young, middle-aged, and older drivers (continued).

Rank Sign Name (Dist) (MUTCD No.)

6. Advance Flagger (W20-7a)

13. Pavement Ends (W8-3a)

14. Bicycle Crossing (W11-1)

15 Campfire (RA-030)

17. Ranger Station (RG-170)

18. Mandatory Seatbelt (R16-1)

19. No Parking (Car)

Mean

$\underline{\text { Redesigned Signs }}$

\begin{tabular}{|c|c|c|c|c|}
\hline $\begin{array}{l}\text { Age } \\
\text { Diff } \\
\end{array}$ & $\frac{\text { Young }}{(\mathrm{SD})}$ & $\begin{array}{l}\text { Middle- } \\
\underline{\text { Aged }} \\
\underline{(\mathrm{SD})}\end{array}$ & $\frac{\text { Older }}{(\mathrm{SD})}$ & $\begin{array}{c}\text { Mean } \\
\text { Dist }\end{array}$ \\
\hline- & $\begin{array}{l}216.7 \\
(65.0)\end{array}$ & $\begin{array}{c}198.0 \\
(57.5)\end{array}$ & $\begin{array}{l}191.9 \\
(53.5)\end{array}$ & 202.2 \\
\hline- & $\begin{array}{l}124.6 \\
(24.4)\end{array}$ & $\begin{array}{l}110.2 \\
(26.4)\end{array}$ & $\begin{array}{l}107.0 \\
(25.7)\end{array}$ & 113.9 \\
\hline $\mathrm{a}$ & $\begin{array}{l}120.9 \\
(24.8)\end{array}$ & $\begin{array}{l}113.5 \\
(24.1)\end{array}$ & $\begin{array}{c}97.1 \\
(2 \quad 1.2)\end{array}$ & 110.8 \\
\hline $\mathrm{a}$ & $\begin{array}{l}132.1 \\
(40.6)\end{array}$ & $\begin{array}{r}96.9 \\
(15.3)\end{array}$ & $\begin{array}{r}98.6 \\
(18.1)\end{array}$ & 109.2 \\
\hline $\mathrm{a}$ & $\begin{array}{l}104.9 \\
(22.6)\end{array}$ & $\begin{array}{c}90.3 \\
(23.2)\end{array}$ & $\begin{array}{c}72.7 \\
(16.1)\end{array}$ & 89.3 \\
\hline $\mathrm{a}$ & $\begin{array}{c}88.1 \\
(16.9)\end{array}$ & $\begin{array}{c}73.4 \\
(22.4)\end{array}$ & $\begin{array}{l}67.3 \\
(21.0)\end{array}$ & 76.3 \\
\hline- & $\begin{array}{c}79.7 \\
(19.0)\end{array}$ & $\begin{array}{c}76.8 \\
(16.8)\end{array}$ & $\begin{array}{c}67.2 \\
(23.8)\end{array}$ & 74.6 \\
\hline $\mathrm{a}$ & 124.0 & 108.4 & 100.3 & 110.9 \\
\hline
\end{tabular}

$\underline{\text { Novel Signs }}$

Rank Sign Name

(Dist) (MUTCD No.)

9. Cross Wind

Age Young $\underline{\text { Aged }}$ Older Mean

Middle-

$\underline{\underline{D i f f}} \quad \underline{\underline{(S D)}} \quad \underline{\underline{(S D)}} \quad \underline{\underline{(S D)}} \quad \underline{\text { Dist }}$

ac $\quad 133.2$

133.4

(16.5)

(27.2)

$113.8 \quad 126.8$

10. Horse Drawn Vehicle a

137.6

127.5

(26.8)

(23.3)

(18.5)

133.2

(27.1)

120.5

(36.7)

108.0

(21.1)

11. Reduced Legibility

128.9

120.4

(29.5)

(45.4)

109.8

(23.8)

12. Truck Entrance

107.9

(22.9)

84.8

(15.8)

96.1

(26.0)

83.5

(17.0)

Mean

128.2

117.3

102.3

124.4

121.2

115.1

92.1

115.9 


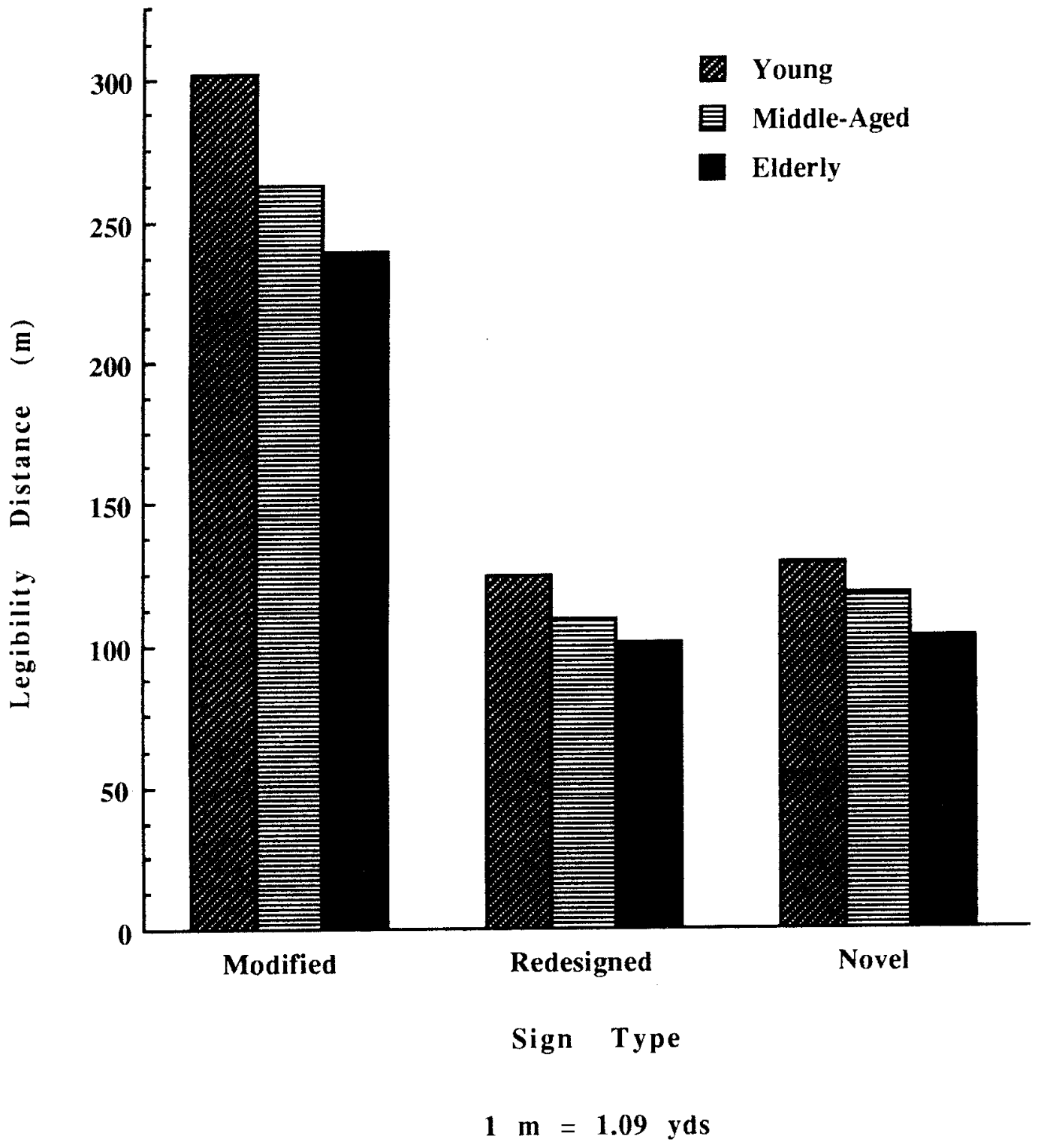

Figure 22. Mean legibility distance $(\mathrm{m})$ of Modified Redesigned, and Novel signs as a function of driver age: daytime legibility (phase 2). 
Table 44. A comparison of overall legibility distance (m) of 14 Modified and Redesigned signs with standard versions across age groups.

$\underline{\text { Modified Signs }}$

\begin{tabular}{|c|c|c|c|c|c|}
\hline & & New & $\underline{\text { Standard }}$ & & $\begin{array}{l}\text { Percent } \\
\text { Increase }\end{array}$ \\
\hline$\underline{\text { Rank }}$ & Sign Names & $\underline{\text { Dist }(\mathrm{m})}$ & $\underline{\text { Dist }(m)}$ & Diff (m) & [Decrease) \\
\hline 1. & Cross Road & 496.4 & 347.2 & $149.2 *$ & $43.0 \%$ \\
\hline 2. & Right Curve & 337.8 & 283.2 & $54.6 *$ & $19.3 \%$ \\
\hline 3. & Hospital & 288.8 & 238.8 & $50.0 *$ & $20.9 \%$ \\
\hline 4. & $\begin{array}{l}\text { Divided Highway } \\
\text { Ends }\end{array}$ & 250.4 & 200.9 & $49.5 *$ & $24.6 \%$ \\
\hline 5 . & Keep Right & 227.6 & 220.0 & 7.6 & $3.5 \%$ \\
\hline 6. & No Parking & 136.1 & 134.0 & 2.1 & $1.6 \%$ \\
\hline 7. & Lane Reduction & 137.2 & 218.9 & $-81.7^{*}$ & $(37.3 \%)$ \\
\hline & Mean & 267.7 & 234.7 & $33.0 *$ & $14.1 \%$ \\
\hline
\end{tabular}

\section{$\underline{\text { Redesigned Signs }}$}

\begin{tabular}{|c|c|c|c|c|c|}
\hline Rank & Sion Names & $\underline{\text { New }}$ & $\frac{\text { Standard }}{\text { Dist }(\mathrm{m})}$ & Diff $(m)$ & $\underline{\text { Percent }}$ \\
\hline$\underline{\text { Rank }}$ & $\underline{\text { Sign Names }}$ & $\underline{\text { Dist (m) }}$ & $\underline{\text { Dist (m) }}$ & $\underline{\text { Dift }(\mathrm{m})}$ & $\underline{\text { (Decrease) }}$ \\
\hline 1. & Advance Flagger & 202.0 & 200.6 & 1.4 & $0.7 \%$ \\
\hline 2. & Pavement Ends & 113.9 & 93.8 & $20.1^{*}$ & $21.4 \%$ \\
\hline 3. & Bicycle Crossing & 110.5 & 146.1 & $-35.6^{*}$ & $(24.4 \%)$ \\
\hline 4. & Campfire & 109.2 & 92.9 & $16.3^{*}$ & $17.6 \%$ \\
\hline 5. & Ranger Station & 89.3 & 66.1 & $23.2^{*}$ & $35.1 \%$ \\
\hline 6. & Seatbelt & 76.3 & 75.7 & 0.6 & $0.8 \%$ \\
\hline 7. & No Parking (Car) & 74.6 & 134.0 & $-59.4^{*}$ & $(44.3 \%)$ \\
\hline & Mean & 110.8 & 115.6 & -4.8 & $(4.2 \%)$ \\
\hline
\end{tabular}

* Mean difference significant at $\mathrm{p}<0.05$ or better. 
Table 45. A comparison of mean legibility distance (m) of 14 Modified and Redesigned signs with standard versions for young drivers.

\begin{tabular}{|c|c|c|c|c|c|}
\hline & \multicolumn{5}{|c|}{$\underline{\text { Modified Signs }}$} \\
\hline & & & & & $\underline{\text { Percent }}$ \\
\hline & & $\underline{\text { New }}$ & $\underline{\text { Standard }}$ & Mean & Increase \\
\hline$\underline{\mathrm{Rank}}$ & $\underline{\text { Sign Name }}$ & Dist (m) & $\underline{\text { Dist }(m)}$ & $\underline{\text { Diff }(m)}$ & (Decrease) \\
\hline 1. & Cross Road & 548.3 & 385.4 & $162.9 *$ & $42.3 \%$ \\
\hline 2. & Right Curve & 387.9 & 270.5 & $117.4 *$ & $43.4 \%$ \\
\hline 3. & Hospital & 307.1 & 257.0 & $50.1 *$ & $19.5 \%$ \\
\hline 4. & Divided Hwy Ends & 286.1 & 228.4 & 57.7 & $25.3 \%$ \\
\hline 5. & Keep Right & 268.9 & 252.3 & 16.6 & $6.6 \%$ \\
\hline 6. & No Parking & 155.4 & 147.6 & 7.8 & $5.3 \%$ \\
\hline 7. & Lane Reduction & 156.2 & 246.4 & $-90.2^{*}$ & $(36.6 \%)$ \\
\hline & Mean & 301.4 & 255.4 & 46.0 & $18.0 \%$ \\
\hline
\end{tabular}

\section{$\underline{\text { Redesigned Signs }}$}

\begin{tabular}{|c|c|c|c|c|c|}
\hline & & $\underline{\text { New }}$ & $\underline{\text { Standard }}$ & Mean & $\begin{array}{l}\text { Percent } \\
\text { Increase }\end{array}$ \\
\hline$\underline{\text { Rank }}$ & $\underline{\text { Sign Names }}$ & Dist (m) & Dist (m) & $\underline{\operatorname{Diff}(\mathrm{m})}$ & (Decrease) \\
\hline 1. & Advance Flagger & 216.1 & 209.2 & 6.9 & $3.3 \%$ \\
\hline 2. & Pavement Ends & 124.6 & 109.9 & 14.7 & $13.4 \%$ \\
\hline 3. & Bicycle Crossing & 121.0 & 159.4 & $-38.4^{*}$ & $(24.1 \%)$ \\
\hline 4. & Campfire & 132.1 & 98.3 & 33.8 * & $34.4 \%$ \\
\hline 5. & Ranger Station & 104.9 & 65.1 & $39.8^{*}$ & $61.1 \%$ \\
\hline 6. & Seatbelt & 88.1 & 92.8 & -4.7 & $(5.1 \%)$ \\
\hline 7. & No Parking (Car) & 79.7 & 147.6 & $-67.9^{*}$ & $(46.0 \%)$ \\
\hline & Mean & 123.8 & 126.0 & -2.2 & $(1.8 \%)$ \\
\hline
\end{tabular}

* Mean difference significant at $\mathrm{p}<0.05$ or better. 
Table 46. A comparison of mean legibility distance (m) of 14 Modified and Redesigned signs with standard versions for middle-aged drivers.

$\underline{\text { Modified Signs }}$

\begin{tabular}{|c|c|c|c|c|c|}
\hline \multirow[b]{2}{*}{$\underline{\text { Rank }}$} & \multirow[b]{2}{*}{ Sign Names } & \multirow{2}{*}{$\begin{array}{l}\text { New } \\
\text { Dist (m) }\end{array}$} & \multirow{2}{*}{$\frac{\text { Standard }}{\underline{\text { Dist }(\mathrm{m})}}$} & Mean & \multirow{2}{*}{$\begin{array}{l}\underline{\text { Percent }} \\
\text { Increase } \\
\underline{\text { (Decrease) }}\end{array}$} \\
\hline & & & & $\underline{\operatorname{Diff}(\mathrm{m})}$ & \\
\hline 1. & Cross Road & 473.2 & 356.8 & $116.4 *$ & $32.6 \%$ \\
\hline 2. & Right Curve & 334.2 & 310.1 & 24.1 & $7.8 \%$ \\
\hline 3. & Hospital & 294.1 & 257.7 & 36.4 & $14.1 \%$ \\
\hline 4. & Divided Highway & 259.3 & 196.5 & $62.8 *$ & $32.0 \%$ \\
\hline & Ends & & & & \\
\hline 5. & Keep Right & 209.5 & 226.4 & -16.9 & $(7.5 \%)$ \\
\hline 6. & No Parking & 132.5 & 138.0 & -5.5 & $(4.0 \%)$ \\
\hline 7. & Lane Reduction & 155.4 & 214.8 & $-59.4^{*}$ & $(27.7 \%)$ \\
\hline & Mean & 265.5 & 242.9 & 22.6 & $9.3 \%$ \\
\hline
\end{tabular}

$\underline{\text { Redeisigiged } \mathrm{n} \quad \mathrm{s}}$

\begin{tabular}{|c|c|c|c|c|c|}
\hline & & $\underline{\text { New }}$ & $\underline{\text { Standard }}$ & Mean & $\begin{array}{l}\text { Percent } \\
\text { Increase }\end{array}$ \\
\hline$\underline{\text { Rank }}$ & $\underline{\text { Sign Names }}$ & Dist (m) & $\underline{\text { Dist }(m)}$ & $\underline{\text { Diff }(m)}$ & /Decrease) \\
\hline 1. & Advance Flagger & 197.9 & 219.7 & -21.8 & $(9.9 \%)$ \\
\hline 2. & Pavement Ends & 110.1 & 96.5 & 13.6 & $14.1 \%$ \\
\hline 3. & Bicycle Crossing & 113.5 & 156.5 & $-43.0 *$ & $(27.5 \%)$ \\
\hline 4. & Campfire & 97.0 & 101.0 & -4.0 & $(4.0 \%)$ \\
\hline 5. & Ranger Station & 90.3 & 75.6 & 14.7 & $19.4 \%$ \\
\hline 6. & Seatbelt & 73.4 & 77.4 & -4.0 & $(5.2 \%)$ \\
\hline 7. & No Parking (Car) & 76.8 & 138.0 & $-61.2^{*}$ & $(44.4 \%)$ \\
\hline & Mean & 108.4 & 123.5 & -15.1 & $(12.2 \%)$ \\
\hline
\end{tabular}

* Mean difference significant at $\mathrm{p}<0.05$ or better. 
Table 47. A comparison of mean legibility distance (m) of 14 Modified and Redesigned signs with standard versions for older drivers.

\section{$\underline{\text { Modified Signs }}$}

\begin{tabular}{|c|c|c|c|c|c|}
\hline \multirow[b]{2}{*}{$\underline{\text { Rank }}$} & \multirow[b]{2}{*}{ Sign Names } & \multirow{2}{*}{$\begin{array}{l}\text { New } \\
\text { Dist (m) }\end{array}$} & \multirow{2}{*}{$\frac{\text { Standard }}{\underline{\text { Dist }(\mathrm{m})}}$} & Mean & $\begin{array}{l}\text { Percent } \\
\text { Increase }\end{array}$ \\
\hline & & & & $\underline{\text { Diff }(m)}$ & Decrease) \\
\hline 1. & Cross Road & 467.6 & 299.4 & $168.2 *$ & $56.2 \%$ \\
\hline 2. & Right Curve & 291.4 & 269.1 & 22.3 & $8.3 \%$ \\
\hline 3. & Hospital & 265.3 & 201.6 & $63.7 *$ & $31.6 \%$ \\
\hline 4. & Divided Highway & 205.9 & 177.8 & 28.1 & $15.8 \%$ \\
\hline & Ends & & & & \\
\hline 5. & Keep Right & 202.0 & 181.1 & 20.9 & $11.5 \%$ \\
\hline 6. & No Parking & 120.4 & 116.3 & 4.1 & $3.5 \%$ \\
\hline 7. & Lane Reduction & 118.5 & 195.4 & $-76.9^{*}$ & $(39.4 \%)$ \\
\hline & Mean & 238.7 & 205.8 & $32.9^{*}$ & $16.0 \%$ \\
\hline
\end{tabular}

$\underline{\text { Redesigned Signs }}$

\begin{tabular}{|c|c|c|c|c|c|}
\hline \multirow[b]{2}{*}{$\underline{\text { Rank }}$} & \multirow[b]{2}{*}{ Sign Names } & New & $\underline{\text { Standard }}$ & \multicolumn{2}{|r|}{$\underline{\text { Percent }}$} \\
\hline & & Dist (m) & $\overline{\text { Dist }(\mathrm{m})}$ & Diff (ml & (Decrease) \\
\hline 1. & Advance Flagger & 191.9 & 173.0 & 18.9 & $10.9 \%$ \\
\hline 2. & Pavement Ends & 107.0 & 75.0 & $32.0 *$ & $42.7 \%$ \\
\hline 3. & Bicycle Crossing & 97.1 & 122.5 & $-25.4 *$ & $(20.7 \%)$ \\
\hline 4. & Campfire & 98.6 & 79.5 & $19.1 *$ & $24.0 \%$ \\
\hline 5. & Ranger Station & 72.7 & 57.5 & $15.2 *$ & $26.4 \%$ \\
\hline 6. & Seatbelt & 67.3 & 56.8 & 10.5 & $18.5 \%$ \\
\hline 7. & No Parking (Car) & 67.2 & 116.3 & $-49.1 *$ & $(42.2 \%)$ \\
\hline & Mean & 100.3 & 97.2 & 3.1 & $3.2 \%$ \\
\hline
\end{tabular}

* Mean difference significant at $\mathrm{p}<0.05$ or better. 
Table 48. Correlations between visual measures and the daytime legibility distance of 19 Modified, Redesigned, and Novel symbol signs among young, middle-aged, and older drivers.

\begin{tabular}{|c|c|c|c|c|}
\hline \multirow[b]{2}{*}{$\underline{\text { Visual Measure }}$} & \multicolumn{3}{|c|}{ Age Group } & \\
\hline & Young & $\begin{array}{l}\text { Middle } \\
\underline{\text { Aged }}\end{array}$ & Older & $\underline{\text { All }}$ \\
\hline Acuity & 0.46 & $0.80 *$ & $0.60 *$ & $0.68 *$ \\
\hline High Cutoff & 0.16 & 0.36 & 0.05 & 0.008 \\
\hline Log of Mean Peak Sensitivity & 0.11 & 0.34 & $0.53 *$ & 0.34 \\
\hline Peak Spatial Frequency & 0.13 & 0.07 & 0.00 & 0.05 \\
\hline
\end{tabular}

*Significant at $\mathrm{p}<.05$ or better

Low-Pass Threshold And Sign Legibility

The means of the two additional young observers who rated each sign in terms of its lowest filter cut-off threshold were averaged to yield an estimation of the low-pass cut-off for each sign. Inter-rater reliability of the two observers, as assessed by Spearman rank correlations, was high

$(\underline{r}=0.94)$. Spearman correlations between the cut-off thresholds for each sign and its legibility distance were also high for each age group (young: $\mathrm{r}=-0.88, \mathrm{p}<0.01$; middle-aged: $\mathrm{r}=-0.87$, $\mathrm{p}<0.01$; older: $\mathrm{r}=-0.86, \mathrm{p}<0.01$, and overall, $\mathrm{r}=-0.85, \mathrm{p}<0.01)$.

\section{Sign Comprehension}

Comprehension of each sign was scored as "correct," "partially correct," or "incorrect," according to the key presented in appendix I. Mean percent correct comprehension scores for each age group and overall are presented in table 49. An age (3) X Gender (2) X Sign Type (3) mixed design ANOVA with Geisser-Greenhouse correction found no significant effects for any of these variables or their interactions. One-way ANOVA's with Tukey HSD comparisons revealed no significant age difference in the comprehension of individual signs.

\section{Predictors Of Sign Comprehension}

Comprehension was not significantly correlated with acuity, peak spatial frequency, educational level, or distance driven per year. There was, however, a significant correlation between total number of years driven and sign comprehension among young observers $(r=0.59$, $\mathrm{p}<0.05)$, a relationship that was not significant among middle-aged drivers, or older participants. 
Table 49. Mean comprehension of 19 Modified, Redesigned, and Novel symbol signs for young, middle-aged, and older drivers.

Sign Name

No. $\quad$ (MUTCD No.)

1. Keep Right

(R4-7)

2. Hospital

(D9-2)

3. No Parking

(R8-3a)

4. Right Curve

(W1-2R)

5. Cross Road

(W2-1)

6. Lane Reduction

Transition

7. Divided Highway

Ends (W6-2)

Mean

Modified Signs

\section{Percentage Total Correct}

Middle-

Young.

Aged

100.00

100.00

Older

Mean

100.00

100.00

100.00

100.00

100.00

100.00

100.00

100.00

100.00

91.66

100.00

94.44

95.37

100.00

100.00

83.33

94.44

95.83

91.66

88.88

92.12

87.50

83.33

83.33

84.72

96.43

96.43

92.85

95.24

$\underline{\text { Redesigned Signs }}$

Percentage Total Correct

Sign Name

$\begin{array}{cl}\text { No. } & \frac{\frac{\text { Sign Name }}{\text { (MUTCD No. })}}{1 .} \quad \begin{array}{l}\text { Pavement Ends } \\ \text { (W8-3a) }\end{array} \\ 2 . & \begin{array}{l}\text { Mandatory Seatbelt } \\ (\text { R16-1) }\end{array}\end{array}$

31

Campfire

(RA-030)

4.

Advance Flagger

(W20-7a)

5. No Parking (Car)

$\frac{\text { Young }}{100.00}$

Middle-

6. Ranger Station

(RG-170)

100.00

$\underline{\text { Aged }}$

100.00

Older

$\underline{\text { Mean }}$

100.00

98.15

100.00

100.00

88.88

96.29

95.83

95.83

86.11

92.59

91.66

91.66

77.77

87.03

87.50

70.83

72.22

76.85

7. Bicycle Crossing

(W11-1)

54.12

75.00

72.22

67.11

89.87

90.47

84.52

88.29 
Table 49. Mean comprehension of 19 Modified, Redesigned, and Novel symbol signs for young, middle-aged, and older drivers (continued).

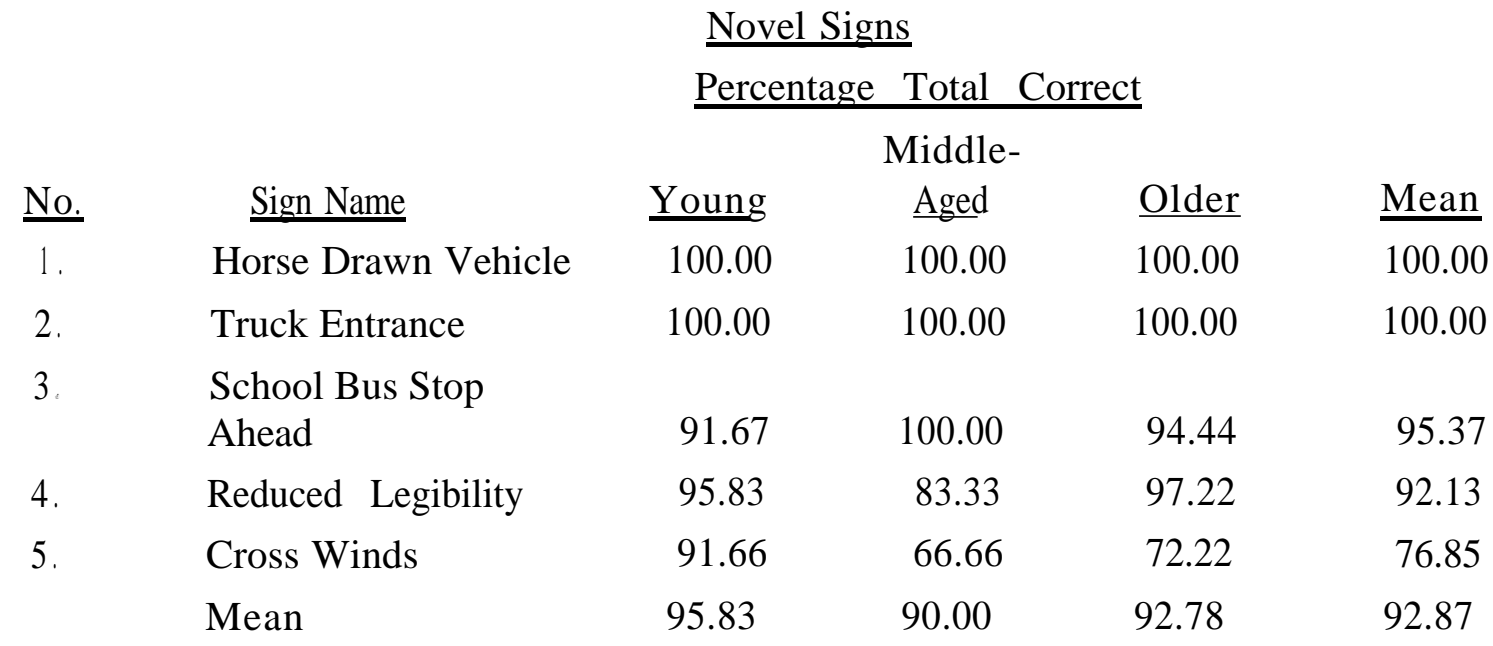

\section{DISCUSSION}

\section{Sign Effectiveness And Driver Age}

Consistent with most previous research, the legibility of symbol signs was inversely related to driver age. ${ }^{(30,57,161)}$ Although the absolute size of the age-related deficit was greater for Modified versions of standard highway signs than Redesigned or Novel signs, that undoubtedly reflected the fact that signs selected for modification were those whose legibility distance was already high. Regardless of sign type, the mean legibility distance of the older group of drivers in this study was about 80 percent that of the young drivers. Although not significantly different from either of the other two age groups overall, the legibility distances of middle-aged drivers averaged about 86 percent those of the young drivers. Based on the 19 signs

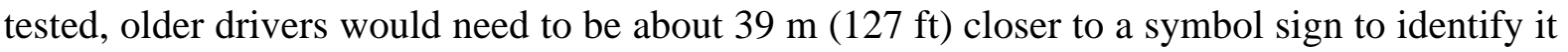
than would a young driver; the corresponding distance for a middle-aged driver would be $23 \mathrm{~m}$ $(75 \mathrm{ft})$. At an assumed travel velocity of $96.6 \mathrm{~km} / \mathrm{h}(60 \mathrm{mi} / \mathrm{h})$, it can be estimated that the average older or middle-aged driver would have $1.4 \mathrm{~s}$ and $0.9 \mathrm{~s}$ less time, respectively, than a young driver to read the average symbol sign. Given the demonstrated decline with age in response speed, an age deficit of this magnitude could have important implications for road safety. ${ }^{(74)}$ It should also be recognized, however, that this problem would be even greater for word-message signs, since their legibility distance is half or less that of symbol signs. ${ }^{(18,19,57)}$

Unlike legibility, comprehension was little affected by driver age. Understanding of the meaning of the symbol signs tested was similarly high for all three age groups for all sign types (between 85 percent and 96 percent). Comprehension of several signs was 100 percent for all three age groups and no significant age differences were observed in the comprehension of any sign. Years of driving experience predicted comprehension only among young drivers, suggesting that drivers increase their knowledge of signs only over the first few years of driving. Overall comprehension levels were high relative to the comprehension studies in phases 1 and 2, 
perhaps reflecting the extended opportunity that drivers had to view and reflect on each sign's meaning during the legibility testing period that preceded the evaluation of comprehension.

\section{Age And Measures Of Spatial Vision}

No age differences on acuity were observed, presumably reflecting the excellent mean presenting acuity of the participants in combination with their refraction to optimal acuity at the test distance. The age difference in contrast sensitivity at the highest spatial frequency tested $(22.5 \mathrm{c} / \mathrm{deg})$ was consistent with the age deficit at higher spatial frequencies that has been observed in several prior studies ${ }^{(123,125)}$.

\section{Visual Predictors Of Legibility}

Despite the excellent acuity of the participants, acuity was a good predictor of the legibility distance of symbol signs regardless of sign type, especially among middle-aged and older drivers. Presumably, the greater utility of acuity as a predictor of legibility distance in these groups reflected the greater inter-individual variability in acuity that remained even after optical correction. The correlation between acuity and legibility distance observed here is somewhat higher than that observed for "improved" symbol signs by Kline and Fuchs, and may reflect the visual demands of the legibility task as it was assessed in the present study. ${ }^{(57)}$ To ensure that observers weren't filling in details from their recollection of familiar signs, thus confounding legibility and familiarity, each sign was increased in size until the observer could actually report all of the basic features in the pre-determined scoring key. This essentially made the task one, which, like acuity, involves the ability to see fine details. Not surprisingly then, composite measures involving contrast sensitivity for lower spatial frequencies were not as robustly related to legibility distance as in studies using different response criteria. ${ }^{(127)}$ In other words, the assessment of legibility was a highly conservative one that assumed that all of the details on a sign were critical to its message.

This issue is exemplified by the SCHOOL BUS STOP AHEAD sign for which two legibility thresholds were established, one in which the small red tail-lights on the back of the bus had to be reported and one in which it did not. In the former case, the mean legibility distance of the sign was $92.1 \mathrm{~m}$ (302.2 A) (see table 43), ranking it sixteenth of the 19 signs tested. Had a report of tail-lights not been required, however, the mean legibility distance of the sign would have been $163.5 \mathrm{~m}(536 \mathrm{ft})$, ranking it seventh overall. Certainly, as drivers become more familiar with a sign and are able to identify it from its lower spatial frequency and/or chromatic characteristics, its effective legibility distance would be increased. It is possible that, despite a systematic effort to avoid such an effect, the prior experience of drivers with of the Modified type may have assisted their identification of such cues.

In consideration of this possibility, in designing signs, it might be useful to distinguish between features of two different types: "critical features" and "educational features." Critical features would be those that would be visible from far away, (e.g., large details, overall shape, color, etc.) and which are sufficient in themselves to convey a sign's message. The least visible critical feature on a sign would determine the distance at which it would be effective.

Educational features, by contrast, would typically be smaller details that inform the approaching driver more fully or specifically about the sign's message, facilitating subsequent identification of 
the sign from its critical features alone. Considering these two types of features somewhat independently would both facilitate the design of symbol signs that are identifiable at a distance sufficient to provide the driver with adequate response time, as well as be informative and aesthetically appealing. For example, the outline of a school bus, adjacent silhouettes of a child, and the "ahead arrow", might be the only critical features of the SCHOOL BUS STOP AHEAD sign that determine its legibility distance. Other added features such as the door, windows, and tail-lights on the bus, however, would verify its identity, so that when seen from far away, it could be identified from its global characteristics. In designing signs using such an approach, considerable emphasis would be placed on optimizing the legibility of critical features, whereas comprehension would be more relevant as its educational features. And certainly, consistent with the findings of Kline and Fuchs, it is possible to significantly increase the legibility distance of symbol signs without making them larger overall. ${ }^{(57)}$

The most direct test of the capacity for improving symbol sign legibility occurred with Modified signs, where changes made were directed primarily at this aspect of their effectiveness. The mean legibility distance was improved using the image-processing approach on six Modified signs for which the basic format of the Standard version was preserved (see table 44). The only reduction occurred on the LANE REDUCTION TRANSITION sign, which by virtue of the addition of a right to left arrow to increase its comprehension, might have been more properly included in the Redesigned category. Overall legibility distance was even increased for most of the Redesigned signs. The two exceptions to this were the BICYCLE CROSSING and NO PARKING (CAR) signs, the legibility distances of which were limited by their relatively small "speed lines" and "curb" details, respectively.

In general, to optimize the legibility of the critical details of a sign's message, contours and contour separations that define these features should be as large and as simple as possible. This can be assured by maximizing their capacity to endure a blur or successive low-pass filtering. In the present study, the low-pass filtration thresholds of signs were excellent predictors (i.e., correlation around 0.9 ) of their legibility distances for young, middle-aged, and older drivers. The benefits of such "low-pass signs" can be seen clearly in a comparison of the CROSS ROAD and SCHOOL BUS STOP AHEAD signs presented in figures 23 and 24, respectively. The message of the CROSS ROAD sign is clear at the lowest filtration level. Many of the critical details in the SCHOOL BUS sign are lost at high levels of low-pass cut-off, and at the lowest level, the sign is virtually indiscriminable. If the relationship between resistance to low-pass filtration and legibility is confirmed in future research, the legibility distance of signs could be optimized in the lab or design studio using image-processing techniques. It also means that a powerful metric is available for predicting a sign's legibility distance at the design stage, obviating the need for extended and expensive testing with human observers of different ages.

It is feasible to design symbol signs whose messages can be immediately comprehended by drivers who are unfamiliar with these signs. Certainly, this was the case for the HORSE DRAWN VEHICLE, TRUCK ENTRANCE, and PAVEMENT ENDS signs, whose meanings were completely understood by all 42 drivers in the study. Not surprisingly, comprehension is not predictable from visual measures, and no metric other than driver testing yet exists for assuring such high levels of comprehension. For example, the BICYCLE CROSSING sign was more poorly comprehended among younger drivers, who frequently reported it to represent a 
"bike path" rather than a "bike crossing." It is important to avoid such unintended ambiguities in designing signs if they are to be comprehended by all of its possible users.

In conclusion, the legibility advantage that symbol signs have over corresponding word message versions can be significantly enhanced and predicted for drivers of all ages by using low-pass filtering techniques. Problems of comprehension, the principal limitation of symbol signs, can be greatly reduced if effective design processes are coupled with a testing program that embraces a broad representative sample of young, middle-aged, and older drivers. 


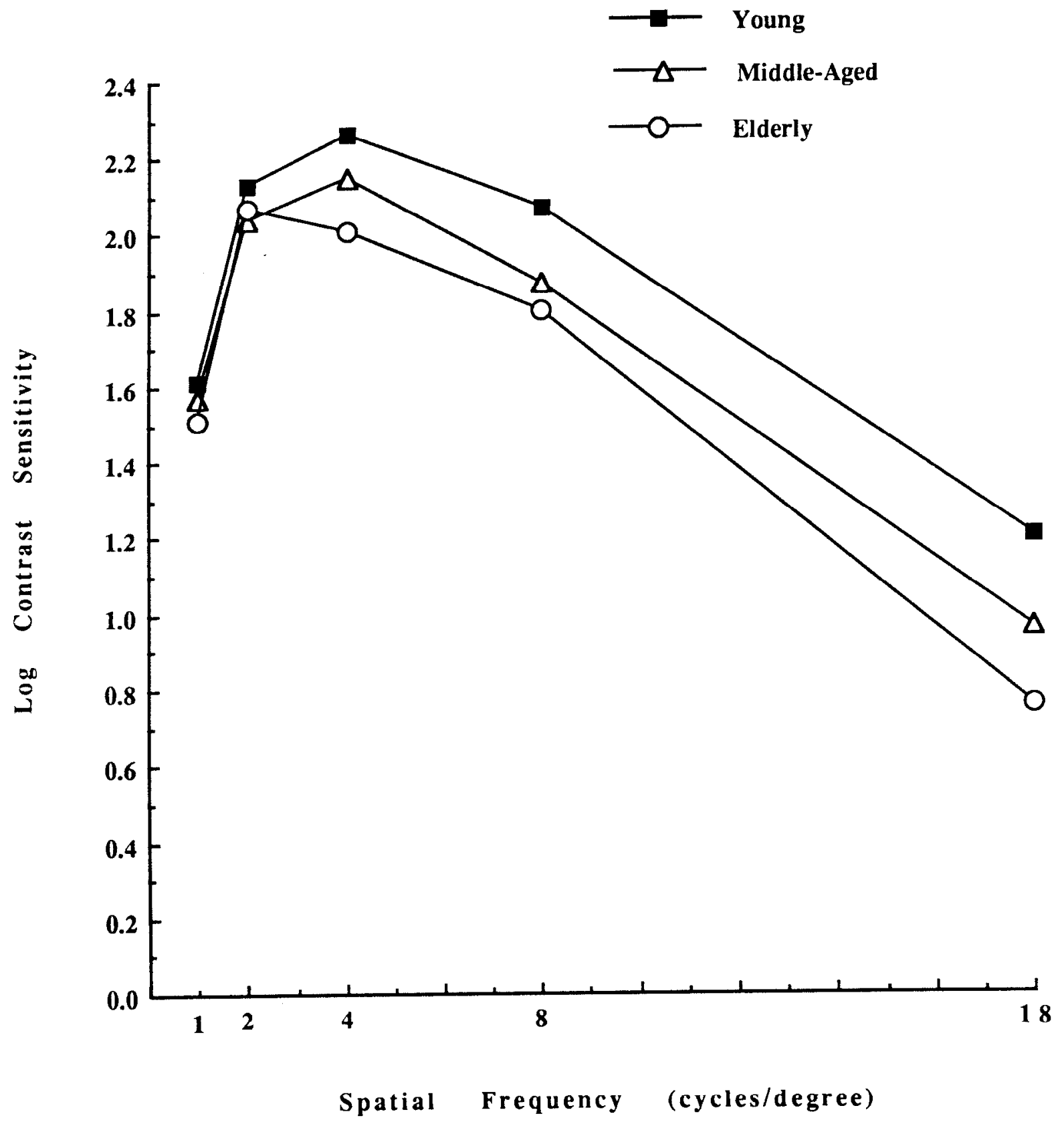

Figure 25. Photopic contrast sensitivity for young, middle-aged, and elderly observers:

nighttime legibility (phase 2). 


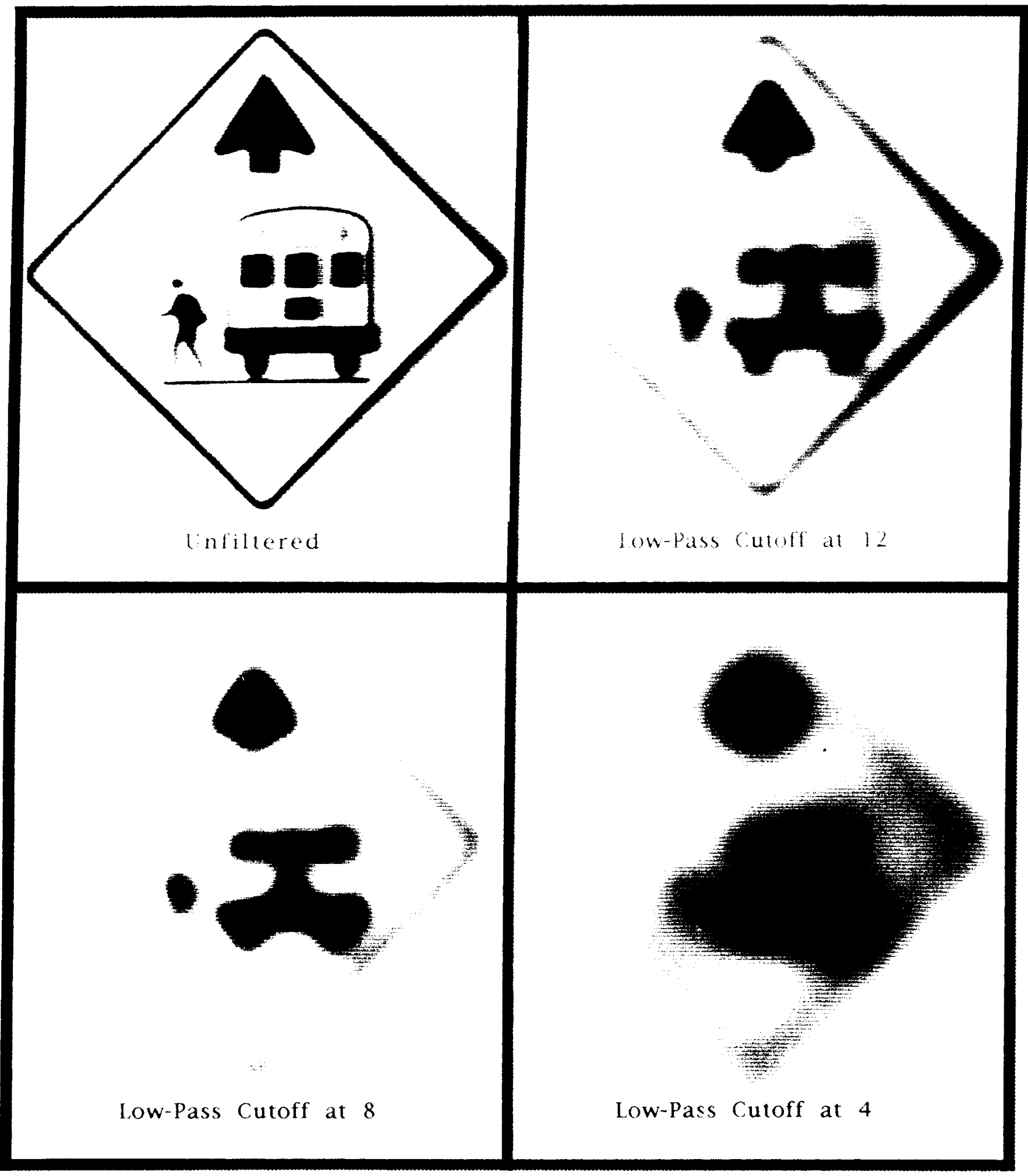

Figure 23. Unfiltered and Butterworth low-pass filtered versions of a low-legibility-distance sign (School Bus Ahead). 


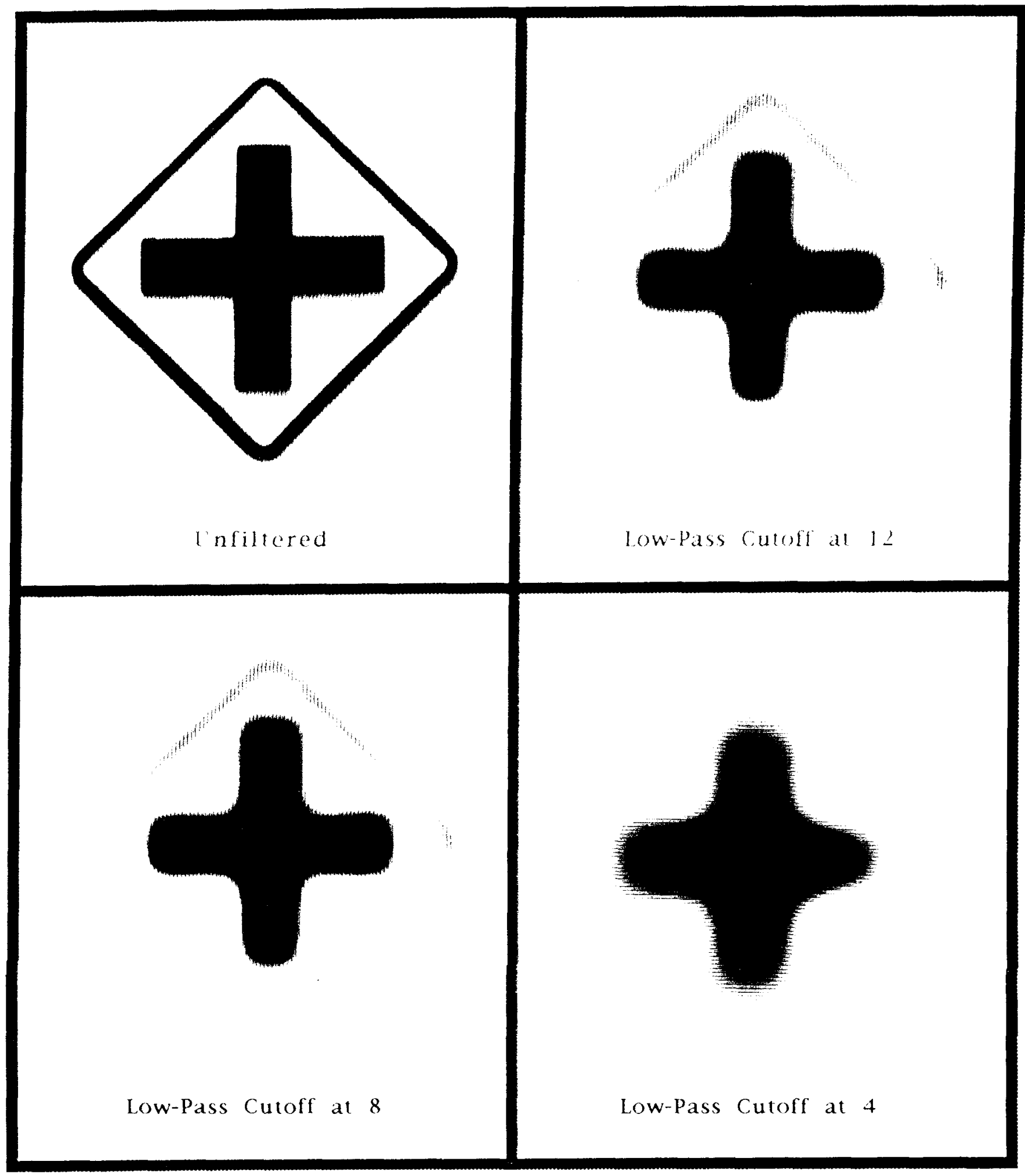

Figure 23. Unfiltered and Butter-worth low-pass filtered versions of a high-legibility-distance sign (Cross Road). 


\section{CHAPTER 12: NIGHTTIME LEGIBILITY OF MODIFIED, REDESIGNED, AND NOVEL SYMBOL HIGHWAY SIGNS}

In phase 1 of the current series of studies, an unexpectedly small decrement in sign visibility was observed when luminance was reduced from 85 to $8.5 \mathrm{~cd} / \mathrm{m}^{2}$. Therefore, in phase 2 , the nighttime luminance level employed was reduced to five $\mathrm{cd} / \mathrm{m}^{2}$. This more challenging viewing condition placed sufficient "stress" upon the photopic visual system to yield significant performance decrements relative to the daytime viewing condition.

\section{METHOD}

\section{Subjects}

Three age groups of active, community-resident drivers were sampled: a young group $(\mathrm{N}=12, \underline{\mathrm{M}}$ age $=19.6$, range 18 to 23$)$, a middle-aged group $(\mathrm{N}=12, \underline{\mathrm{M}}$ age $=47.7$, range 40 to $56)$ and an older group $(\mathrm{N}=18, \mathrm{M}$ age $=74.4$, range 66 to 87$)$. The young subjects were recruited from the student population of the University of South Dakota and the middle-aged and older subjects were recruited from community service organizations in Vermillion, SD. Visual health of the three age groups, as indexed by photopic contrast sensitivity, was representative of known population values (see table 50). All subjects completed the experimental protocols.

\section{Stimuli And Apparatus}

Binocular photopic $\left(85 \mathrm{~cd} / \mathrm{m}^{2}\right)$ acuity was measured using a customized Landolt $\mathrm{C}$ chart. This chart divided the acuity range between 20/40 to 20/10 (Snellen) into 15 equivalently spaced optotype intervals-yielding a highly precise estimate of spatial resolving power. Contrast sensitivity was assesses using a CRT-based analog of the Vistech VCTS 6500 far chart (used at the University of Calgary laboratory). The advantage of the CRT-based technique was that more precise estimates of contrast sensitivity could be obtained. Contrast sensitivities were measured at each of 5 spatial frequencies: 1, 2, 4, 8, and 18 cycles per degree of visual angle (c/deg) at a space-averaged luminance level of $22 \mathrm{~cd} / \mathrm{m}^{2}$.

The test stimuli consisted of 18 symbol signs engineered for optimal visibility at the University of Calgary. They were presented in their regulation colors using an Apple IIci computer equipped with an Apple $330 \mathrm{~mm}$ (13 in) RGB monitor. White areas of the screen were maintained at $5 \mathrm{~cd} / \mathrm{m}^{2}$. A viewing distance of $5.5 \mathrm{~m}(18 \mathrm{ft})$ was maintained by the use of a head/chin rest.

\section{Procedure}

After completion of the informed consent procedure and the driver background questionnaire, photopic acuity and contrast sensitivity functions were collected. The laboratory was then darkened and the recognition size/distance thresholds for the practice and test symbol signs were collected using the same procedure detailed in the Method section of the Daytime Legibility study. 
Table 50. Vision assessment parameters as a function of age: nighttime legibility study (phase 2).

\begin{tabular}{lccc} 
& \multicolumn{1}{c}{$\underline{\text { Age group }}$} & \\
\cline { 2 - 3 } & 2.30 & $\underline{\text { Middle-Aged }}$ & $\underline{\text { Old }}$ \\
Hog Peak CS & 8.45 & 2.18 & 2.11 \\
High Frequency Cutoff (c/deg) & 0.692 & 7.47 & 6.64 \\
Acuity (minarc) & & 0.892 & 1.117
\end{tabular}

\section{RESULTS}

\section{Contrast Sensitivity}

The contrast sensitivity functions collected from each of the age groups are plotted in figure 25. An Age (3) by Spatial Frequency (5) ANOVA was performed upon the log-transformed contrast sensitivity data. The results of the ANOVA revealed a significant main effect of $\operatorname{Age}[F(2,39)=8.26, p<0.001]$, as well as a significant Age by Spatial Frequency interaction $[\mathrm{F}(7.4,144)=2.06, \mathrm{p}<0.051$. In summary, an age-related reduction in contrast sensitivity emerged as the spatial frequency of the sine wave target was increased.

Simple-effects analyses revealed that significant age-related reductions in contrast sensitivity occurred at 4, 8, and $18 \mathrm{c} / \mathrm{deg}$. No significant age differences in contrast sensitivity were observed for the two lowest spatial frequency targets (viz., one and two c/deg). Age group contrasts indicated that the contrast sensitivity of the oldest group was significantly different from both the young and middle-aged groups across this same range of spatial frequencies. No significant differences between the young and middle-aged groups appeared at any of the spatial frequencies examined. The main effect of Spatial Frequency, as is always the case, was highly significant $[\mathrm{F}(3.7,144)=162.5, \mathrm{p}<0.0001]$. Contrast sensitivity peaked between two and four $\mathrm{c} / \mathrm{deg}$ and rapidly declined at both higher and lower spatial frequencies. The nature of this Spatial Frequency trend and the Age by Spatial Frequency interaction effects are apparent in figure 25.

\section{Sign Legibility Distance}

The minimum size needed by the observers to meet the visibility criterion for each sign was converted to a legibility distance index using the formula: Legibility distance $(\mathrm{m})=$ (Regulation Sign Size/ Thresholder Size) x Test Viewing Distance. Average legibility distances for each sign as a function of age are enumerated in table 51. Average legibility distances as a function of age group and sign category (Modified, Redesigned and Novel) can be seen in figure 26. As with the no-glare condition in the earlier study, legibility distances decreased with increasing age.

The legibility distance data were analyzed using an Age (3) by Sign (18) ANOVA. Both the main effects of Age $[F(2,39)=20.47, p<0.0001]$, Sign $[F(4.75,185)=265.9$, p < 0.0001], as well as the Age by Sign interaction $[\mathrm{F}(9.51,185)=5.23, \mathrm{p}<0.0001]$ were highly significant. Simple-effects analyses revealed that the Signs factor was highly significant for all three age 
groups: Young $[\mathrm{F}(4.75,185)=100.9, \mathrm{p}<0.001]$, Middle-aged $[\mathrm{F}(4.75,185)=95.2, \mathrm{p}<$ $0.0001]$, and Older $[\mathrm{F}(4.75,185)=73.3, \mathrm{p}<0.0001]$. 


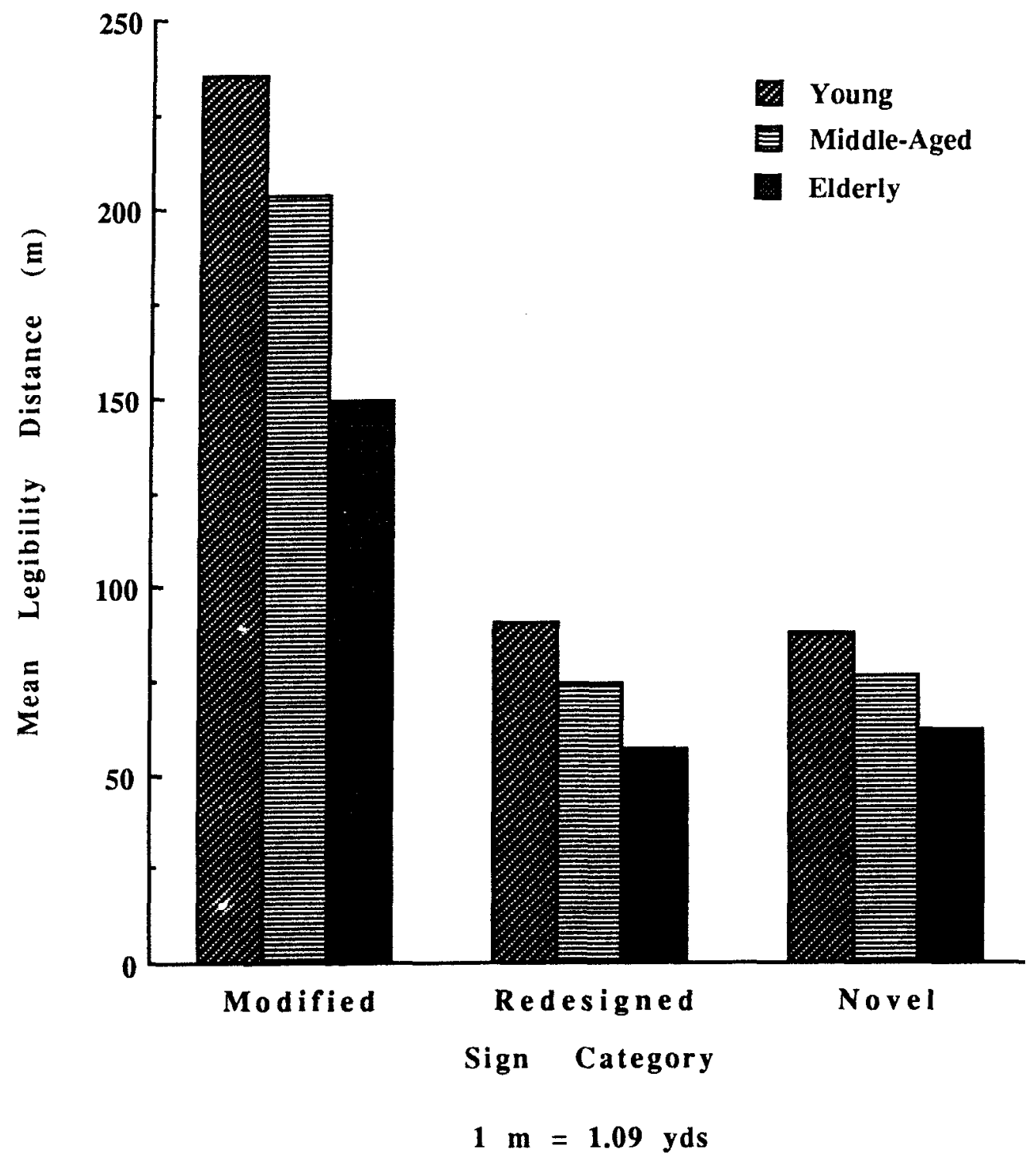

Figure 26. Mean nighttime legibility distances of different sign categories as a function of driver age: nighttime legibility (phase 2). 
Table 51. Highway signs ranked on legibility distance (m) for low luminance condition: nighttime legibility study (phase 2).

\section{$\underline{\text { Modified Signs }}$}

\begin{tabular}{|c|c|c|c|c|c|c|c|}
\hline USD & $\mathrm{UC}$ & Sign & Age & & Middle- & & All \\
\hline Rank $\S$ & $\underline{\text { Rank }}$ & Description & $\underline{\text { Diff }}$ & $\underline{\text { Young }}$ & Aged & $\underline{\text { Older }}$ & Groups \\
\hline 1 & 1 & $\begin{array}{l}\text { Cross Road } \\
\text { (W2-1) }\end{array}$ & $\mathrm{ac}$ & $\begin{array}{l}377.9 \\
(54.8)\end{array}$ & $\begin{array}{l}382.0 \\
(82.0)\end{array}$ & $\begin{array}{l}267.9 \\
(72.3)\end{array}$ & $\begin{array}{l}331.9 \\
(89.0)\end{array}$ \\
\hline 2 & 2 & $\begin{array}{l}\text { Right Curve } \\
\text { (W1-2R) }\end{array}$ & $\mathrm{ac}$ & $\begin{array}{l}314.6 \\
(77.3)\end{array}$ & $\begin{array}{l}271.6 \\
(58.9)\end{array}$ & $\begin{array}{l}192.1 \\
(65.8)\end{array}$ & $\begin{array}{l}249.8 \\
(84.6)\end{array}$ \\
\hline 3 & 4 & $\begin{array}{l}\text { Divided Hwy Ends } \\
\text { (W6-2) }\end{array}$ & $a b$ & $\begin{array}{l}233.8 \\
(66.5)\end{array}$ & $\begin{array}{l}155.8 \\
(76.9)\end{array}$ & $\begin{array}{l}132.8 \\
(44.9)\end{array}$ & $\begin{array}{l}168.2 \\
(77.5)\end{array}$ \\
\hline 4 & 3 & $\begin{array}{l}\text { Hospital } \\
\text { (D9-2) }\end{array}$ & $\mathrm{a}$ & $\begin{array}{l}188.8 \\
(63.3)\end{array}$ & $\begin{array}{l}172.3 \\
(68.1)\end{array}$ & $\begin{array}{l}130.5 \\
(46.8)\end{array}$ & $\begin{array}{l}159.1 \\
(70.2)\end{array}$ \\
\hline 6 & 5 & $\begin{array}{l}\text { Keep Right } \\
\text { (R4-7) }\end{array}$ & $\mathrm{a}$ & $\begin{array}{l}164.5 \\
(78.1)\end{array}$ & $\begin{array}{l}130.1 \\
(48.7)\end{array}$ & $\begin{array}{l}106.5 \\
(52.1)\end{array}$ & $\begin{array}{l}129.8 \\
(75.5)\end{array}$ \\
\hline \multirow[t]{2}{*}{7} & 8 & $\begin{array}{l}\text { No Parking } \\
\text { (R8-3a) }\end{array}$ & $\mathrm{ac}$ & $\begin{array}{l}131.0 \\
(30.4)\end{array}$ & $\begin{array}{l}112.9 \\
(30.1)\end{array}$ & $\begin{array}{c}67.0 \\
(23.8)\end{array}$ & $\begin{array}{c}98.4 \\
(39.1)\end{array}$ \\
\hline & & Mean & & 235.1 & 204.1 & 149.5 & 189.5 \\
\hline
\end{tabular}

Notes:

USD Rank - Ordinal ranking of signs based upon the performance of the young group

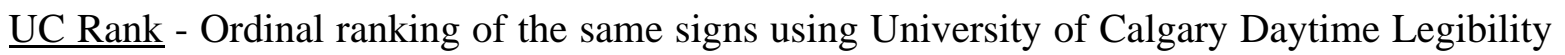
data.

Age Diff (i.e. post hoc comparison) Legend:

a Young $>$ Older $(p<0.001)$

b Young $>$ Middle-aged $(p<0.001)$

c Middle-aged > Older $(p<0.001)$

$\S$ Spearman Rank-Order Correlation (Rho) analysis of the University of Calgary daytime versus USD nighttime legibility data across signs yielded a value of 0.965 ( $p<0.0001)$.

( ) Standard deviations appear in parentheses 
Table 51. Highway signs ranked on legibility distance (m) for low luminance condition: nighttime legibility study (phase 2) (continued).

$\underline{\mathrm{Redesign}}$ e d

\begin{tabular}{|c|c|c|c|c|c|c|c|}
\hline USD & UC & Sign & Age & & Middle- & & All \\
\hline$\underline{\mathrm{Rank}} \S$ & $\underline{\mathrm{Rank}}$ & $\underline{\text { Description }}$ & $\underline{\text { Diff }}$ & Young: & $\underline{\text { Aged }}$ & $\underline{\text { Older }}$ & $\underline{\text { Groups }}$ \\
\hline 5 & 6 & $\begin{array}{l}\text { Advance Flagger } \\
(\mathrm{W} 20-7 \mathrm{a})\end{array}$ & $\mathrm{a}$ & $\begin{array}{l}187.2 \\
(68.8)\end{array}$ & $\begin{array}{l}156.9 \\
(49.4)\end{array}$ & $\begin{array}{l}125.8 \\
(40.4)\end{array}$ & $\begin{array}{l}152.2 \\
(57.2)\end{array}$ \\
\hline 11 & 13 & $\begin{array}{l}\text { Pavement Ends } \\
\text { (W8-3a) }\end{array}$ & $a b$ & $\begin{array}{c}86.1 \\
(24.5)\end{array}$ & $\begin{array}{l}63.9 \\
(9.2)\end{array}$ & $\begin{array}{c}60.8 \\
(19.4)\end{array}$ & $\begin{array}{c}68.9 \\
(21.5)\end{array}$ \\
\hline 13 & 14 & $\begin{array}{l}\text { Bicycle Crossing } \\
\text { (W11-1) }\end{array}$ & $\mathrm{ac}$ & $\begin{array}{c}83.4 \\
(17.2)\end{array}$ & $\begin{array}{c}67.1 \\
(13.5)\end{array}$ & $\begin{array}{c}48.5 \\
(10.9)\end{array}$ & $\begin{array}{c}63.8 \\
(19.1)\end{array}$ \\
\hline 16 & 17 & $\begin{array}{l}\text { Ranger Station } \\
(\mathrm{RG}-170)\end{array}$ & $\mathrm{ac}$ & $\begin{array}{l}65.1 \\
(21.9)\end{array}$ & $\begin{array}{c}59.6 \\
(12.0)\end{array}$ & $\begin{array}{c}42.7 \\
(14.6)\end{array}$ & $\begin{array}{c}53.9 \\
(16.8)\end{array}$ \\
\hline 17 & 15 & $\begin{array}{l}\text { Campfire } \\
\text { (RA-030) }\end{array}$ & $\mathrm{ac}$ & $\begin{array}{c}59.6 \\
(11.4)\end{array}$ & $\begin{array}{c}48.7 \\
(13.8)\end{array}$ & $\begin{array}{l}30.6 \\
(9.8)\end{array}$ & $\begin{array}{c}44.0 \\
(16.8)\end{array}$ \\
\hline \multirow[t]{3}{*}{18} & 18 & $\begin{array}{l}\text { Mandatory Seat } \\
\text { Belt (R16-1) }\end{array}$ & $\mathrm{ac}$ & $\begin{array}{l}58.5 \\
(17.9)\end{array}$ & $\begin{array}{l}48.1 \\
(12.4)\end{array}$ & $\begin{array}{l}31.6 \\
(9.8)\end{array}$ & $\begin{array}{c}44.0 \\
(17.4)\end{array}$ \\
\hline & & Mean & & 90.0 & 74.1 & 56.7 & 71.1 \\
\hline & \multicolumn{7}{|c|}{$\underline{\text { Novel Signs }}$} \\
\hline USD & UC & Sign & Age & & Middle- & & All \\
\hline$\underline{\mathrm{Rank}} \S$ & $\underline{\mathrm{Rank}}$ & $\underline{\text { Description }}$ & $\underline{\text { Diff }}$ & Young & $\underline{\text { Aged }}$ & $\underline{\text { Older }}$ & $\underline{\text { Groups }}$ \\
\hline 8 & 10 & $\begin{array}{l}\text { Horse Drawn } \\
\text { Vehicle }\end{array}$ & $\mathrm{a}$ & $\begin{array}{l}103.6 \\
(29.3)\end{array}$ & $\begin{array}{c}87.9 \\
(17.7)\end{array}$ & $\begin{array}{l}72.9 \\
(21.0)\end{array}$ & $\begin{array}{c}85.9 \\
(25.8)\end{array}$ \\
\hline 9 & 9 & Crosswinds & $\mathrm{a}$ & $\begin{array}{l}97.6 \\
(26.0)\end{array}$ & $\begin{array}{c}80.9 \\
(19.0)\end{array}$ & $\begin{array}{c}69.6 \\
(14.0)\end{array}$ & $\begin{array}{l}80.8 \\
(22.3)\end{array}$ \\
\hline 10 & 7 & Lane Reduction & & $\begin{array}{l}95.1 \\
(30.6)\end{array}$ & $\begin{array}{l}76.6 \\
(11.4)\end{array}$ & $\begin{array}{l}71.3 \\
(30.5)\end{array}$ & $\begin{array}{c}80.4 \\
(28.3)\end{array}$ \\
\hline 12 & 11 & Fog & $\mathrm{a}$ & $\begin{array}{c}85.2 \\
(22.7)\end{array}$ & $\begin{array}{c}81.2 \\
(31.9)\end{array}$ & $\begin{array}{l}58.8 \\
(19.6)\end{array}$ & $\begin{array}{l}72.7 \\
(26.9)\end{array}$ \\
\hline 14 & 12 & Truck Entrance & $\mathrm{ac}$ & $\begin{array}{c}78.7 \\
(29.2)\end{array}$ & $\begin{array}{c}68.0 \\
(27.8)\end{array}$ & $\begin{array}{c}44.7 \\
(10.7)\end{array}$ & $\begin{array}{c}61.1 \\
(26.6)\end{array}$ \\
\hline 15 & 16 & School Bus Stop & $\mathrm{a}$ & $\begin{array}{c}66.7 \\
(15.0)\end{array}$ & $\begin{array}{l}62.2 \\
(7.3)\end{array}$ & $\begin{array}{c}53.6 \\
(10.2)\end{array}$ & $\begin{array}{c}59.8 \\
(12.3)\end{array}$ \\
\hline & & Mean & & 87.8 & 76.1 & 61.8 & 73.5 \\
\hline
\end{tabular}


Similar results were obtained for the simple-effects analyses of the Age factor. Significant age effects $(\mathrm{p}<0.05)$ were obtained for every sign. Simple-simple age effects analyses were performed in order to isolate the specific nature of the age effect (i.e., young vs. middle-aged vs. older) for each of the 18 test signs. The results of these statistical comparisons are summarized in the column labeled "Age Diff" on table 51. In general, better legibility distances in the young group (i.e., the "best" signs) were associated with the largest absolute age-related performance decrements. This pattern of results replicated the general findings observed in phase 1 of the study.

\section{Visual Predictors Of Sign Legibility}

Photopic acuity measures and complete contrast sensitivity functions (CSF's) collected under low photopic luminance conditions were available for all of the subjects participating in the current study. Preliminary correlational analyses were performed in order to assess the degree to which variations in visual ability, as indexed by acuity and the CSF, might be associated with concomitant variations in overall highway sign visibility performance. In order to simplify the analyses based upon contrast sensitivity, the CSF's obtained from each subject were fitted with a 3rd order polynomial which was used to parameterize the CSF into two descriptive indices: the highest contrast sensitivity value observed ("peak CS"), and the half-amplitude high spatial frequency cutoff ("high cutoff'). The mean values observed in each age group for each of these CSF parameters, as well as photopic visual acuity, are presented in table 50. Pearson product-moment correlations were calculated between average legibility distance (collapsed across all signs) and each of the following measures of basic visual function: acuity, log peak CS, and high spatial frequency cutoff. The resulting correlation coefficients are summarized in table 52.

Table 52. Mean overall sign legibility distance correlational analyses.

$$
\text { (all subjects: } \mathrm{N}=40 \text { ) }
$$

$\begin{array}{lll} & \begin{array}{l}\text { Correlation } \\ \text { coefficient }\end{array} & \underline{p \text { value }} \\ \text { Age } & -0.718 & <0.001 \\ \text { Acuity (minarc) } & -0.595 & <0.001 \\ \text { Log Peak Contrast Sensitivity } & 0.318 & <0.05 \\ \text { High Spatial Frequency Cutoff } & 0.578 & <0.001\end{array}$

For the overall sample, adult age had the highest correlation with average sign legibility distance $(r=-0.718)$. Legibility distance decreased as age increased. Although not as strongly correlated as the age factor, both acuity $(\mathrm{r}=-0.595)$ and the high spatial frequency cutoff of the CSF ( $r=0.578)$ were significantly related to overall sign visibility. Increases in the minimum angle of spatial resolution (in minarc) were associated with decreases in sign legibility distance, while increases in the cutoff spatial frequency were associated with improvements in the maximum legibility distance. Log peak contrast sensitivity was only weakly associated with legibility distance $(r=0.318)$. 


\section{Daytime Versus Nighttime Legibility}

Group mean legibility distances obtained under daytime (Calgary) versus nighttime (South Dakota) conditions were compared and depicted a loss of visibility at low luminance. Hence, the reduction in the nighttime test luminance from $8.5 \mathrm{~cd} / \mathrm{m}^{2}$ in phase 1 to $5 \mathrm{~cd} / \mathrm{m}^{2}$ in phase 2 appeared to successfully "challenge" the photopic visual recognition system.

\section{DISCUSSION}

Strong and systematic differences in legibility distance were found across the 18 symbol signs examined in this study. Consistent adult age differences were also superimposed upon this sign effect. Older adults demonstrated significant reductions in legibility distance for every stimulus except the LANE REDUCTION sign. In addition to these young vs. older performance decrements, marked middle-aged vs. older group differences were observed on many of the signs as well (i.e., CROSSROAD, RIGHT CURVE, NO PARKING, BICYCLE CROSSING, RANGER STATION, CAMPFIRE, SEAT BELTS and TRUCK ENTRANCE). Age-related deficits in the middle-aged relative to the young group were observed for only two signs: DIVIDED HIGHWAY ENDS and PAVEMENT ENDS.

The rank-ordering of the signs according to legibility distance remained essentially the same across all three age groups. This uniformity of trend demonstrates the reliability of the threshold estimation procedures employed in this and related studies. The near-perfect rank-order correlation observed between the legibility distance of the 18 test signs under nighttime vs. daytime conditions (Spearman's rho $=0.965)$ suggests that differences in nighttime visibility will yield to straightforward modeling in subsequent research. For example, nighttime visibility of the middle-aged or older, for a given sign, would appear to be predictable on the basis of knowing the daytime visibility of the young using a simple multiplicative formula such as:

$$
\text { nighttime }_{\text {visibility }_{\text {older }}}=\mathrm{k} \times \text { daytime }_{\text {visibility }_{\text {young }}}
$$

Analysis of the contrast sensitivity data revealed a pattern of age differences consistent with the findings of previous research. CSF in the older driver was significantly depressed at all spatial frequencies above $2 \mathrm{c} / \mathrm{deg}$ (see figure 24).

As was the case for the daytime conditions, photopic acuity and its conceptually related CSF parameter, high cutoff spatial frequency, were found to be good predictors of overall sign visibility. However, "peak CS" appeared to contribute little to the prediction of overall sign visibility. This later result was disappointing and not consistent with our original expectations regarding the application of the CSF and Fourier-modeling in the development of symbol highway signs. Part of the reason for these results may be based upon the "criteria" used to define the threshold legibility distance for each sign. Most signs had multiple criteria. Typically, observers could recognize some of the criterion details in a sign at distances far greater than the most challenging detail. In fact, observers could often identify the overall highway sign long before they could describe the most rigorous criterion feature. Thus, the threshold was determined by the most spatially challenging (often highest spatial frequency) component of the 
sign. Such a scheme, it can be argued, biased the prediction of visibility away from measures such as peak CS and toward high spatial frequency cutoff and/or acuity measures. 
195 


\section{CHAPTER 13: NIGHTTIME LEGIBILITY OF MODIFIED, REDESIGNED, AND NOVEL SYMBOL HIGHWAY SIGNS IN THE PRESENCE OF PERIPHERAL GLARE}

In phase 1 of the current series of studies, an unexpectedly small decrement in sign visibility was observed when luminance was reduced from 85 to $8.5 \mathrm{~cd} / \mathrm{m}^{2}$. Therefore, in phase 2 , the nighttime luminance level employed was reduced to $5 \mathrm{~cd} / \mathrm{m}^{2}$. This more challenging viewing condition placed sufficient "stress" upon the photopic visual system to yield significant performance decrements relative to the daytime viewing condition.

Similarly, phase 1 failed to demonstrate consistent age differences in the effects of glare. Performance trends suggested that this failure may have been due to long-term adaptation to a constant glare source. Phase 2 adopted the use of a "transient" glare source aimed at minimizing the potential for such long-term adaptation effects, and at increasing the similarity of the laboratory glare exposure to the types of encounters experienced by nighttime drivers.

\section{METHOD}

\section{Subjects}

Three age groups of active, community-resident drivers were sampled: a young group $(\mathrm{N}=12, \underline{\mathrm{M}}$ age $=19.8$, range 18 to 24$)$, a middle-aged group $(\mathrm{N}=12, \underline{\mathrm{M}}$ age $=47.5$, range 40 to $63)$ and an older group $(\mathrm{N}=18, \underline{\mathrm{M}}$ age $=73: 9$, range 65 to 84$)$. The young subjects were recruited from the student population of the University of South Dakota and the middle-aged and older subjects were recruited from community service organizations in Vermillion, SD.

However, three of the 18 older subjects were excluded from the data analysis because they could not see the highway sign targets in the presence of the glare source. Visual status of the three age groups, as indexed by photopic acuity and contrast sensitivity functions, was representative of known population values (see table 53).

Table 53. Vision assessment parameters as a function of age: nighttime-with-glare legibility study (phase 2).

$\begin{array}{lll}\text { Parameter } & \text { Young } & \underline{\text { Age group }} \\ & \underline{\text { Middle-aged }} \quad \underline{\text { Older }}\end{array}$

Glare Absent

Log Peak CS

$2.21-2.31$

High Frequency Cutoff (c/deg)

7.79

Acuity (minarc)

0.79

0.63

$\underline{\text { Glare Present }}$

Log Peak CS

1.82

1.81

High Frequency Cutoff (c/deg)

9.06

8.49

6.64 


\section{Stimuli And Apparatus}

Binocular photopic $\left(85 \mathrm{~cd} / \mathrm{m}^{2}\right)$ acuity and contrast sensitivities were measured as in the preceding study. Contrast sensitivity was assessed both with and without the presentation of a dual peripheral glare source. These glare sources consisted of high-diffusion 60 watt circular flood lamps mounted $0.3^{\circ}$ (edge-to-edge) to the left and right of the $2.0^{\circ}$ (wide) by $1.6^{\circ}$ (high) CRT display area. Both lamps subtended a visual angle of $0.6^{\circ}$ and were mounted with shielding materials which prevented the extra ocular mixing of light emitted from the stimulus CRT and glare sources, respectively. The CRT display equivalent luminance of each glare source was $100,000 \mathrm{~cd} / \mathrm{m}^{2}$, yielding a combined illuminance of 74 lux measured at the entrance pupil to the eye, approximating the light level generated by a passing oncoming automobile with high-beam head lamps.

The test stimuli consisted of the 18 symbol signs used in the preceding study. Sign stimuli were presented in their regulation colors using an Apple IIci computer equipped with a $330 \mathrm{~mm}$ (13 in) Apple RGB monitor. The white background areas of the stimulus screen were maintained at $5 \mathrm{~cd} / \mathrm{m}^{2}$. A viewing distance of $5.5 \mathrm{~m}(18 \mathrm{ft})$ was maintained by the use of a head/chin rest.

Sign visibility measures were collected in the presence of a bright peripheral glare source located to the left of the stimulus display monitor. This glare source consisted of a single 40 watt high-diffusion incandescent lamp which subtended a visual angle of $0.6^{\circ}$. The lamp was mounted in a housing which prevented the extra ocular mixing of the glare light with that emitted from the stimulus display monitor. CRT display equivalent luminance of the glare source was 12 $000 \mathrm{~cd} / \mathrm{m}^{2}$ (nominal). At the entrance pupil to the eye, the illuminance of the glare source was 8 lux, approximating the intensity of a pair of automobile head lamps viewed at a distance of 30.5 $\mathrm{m}(100 \mathrm{ft})$.

\section{Procedure}

After completion of the informed consent procedure, subjects filled out the standard driving experience questionnaire (see previous studies for details). At this point, their photopic acuity was measured. The laboratory was then darkened for the remainder of the session. Contrast sensitivity functions were collected both with and without the presence of the peripheral glare source described above. Glare-absent/glare-present CSF assessment order was fully counterbalanced across subjects. Next, the recognition size/distance thresholds for the practice and test symbol signs were collected using the same procedure detailed in the Method section of the Daytime Legibility study. However, the temporal duty cycle of the stimulus was changed from that of a static presentation mode to a dynamic one. That is, the stimuli were presented with a 3-s "on"/2-s "off" duty cycle. During the 3-s "on" period, both the stimulus sign and the glare source were presented. Both the stimulus sign and the glare source were absent during the 2-s "off" period. During the "glare-off" periods the blank CRT screen remained on, so the only source of illumination was the screen. The nominal luminance of the surrounding room surfaces during this cycle was $0.05 \mathrm{~cd} / \mathrm{m}^{2}$. The transient nature of this dynamic presentation mode was implemented to counteract long-term adaptation effects to static glare source which appeared to occur in phase 1 of the current research project, and to emulate more closely glare presentations as they would naturally occur on the road while driving at night. 


\section{RESULTS}

\section{Contrast Sensitivity}

The mean contrast sensitivity functions collected from each of the age groups under the glare-absent and glare-present conditions are plotted in figures 27 and 28, respectively. An Age (3) by Glare (2) Condition (i.e., glare absent vs. present) by Spatial Frequency (5) ANOVA was performed upon the log-transformed contrast sensitivity data. The results of the ANOVA revealed significant main effects of $\operatorname{Age}[F(2,35)=17.6, p<0.0001]$, Glare Condition $[F(1,35)$ $=557.1, \mathrm{p}<0.0001]$ and Spatial Frequency $[\mathrm{F}(3.5,122.9)=227.6, \mathrm{p}<0.0001]$. The Age by Glare Condition $[\mathrm{F} 2,35)=25.2, \mathrm{p}<0.0001]$ interaction was also significant. That is, the magnitude of age difference in contrast sensitivity was exacerbated with the introduction of a dual glare source. Finally, the Age by Spatial Frequency interaction $[F(7,122.9)=4.1$, $p<$ 0.004 ] was significant, the age-related decrement in contrast sensitivity tending to be greater at the higher spatial frequencies. Neither the Glare Condition by Spatial Frequency nor the triple interaction approached statistical significance. All of the statistical effects involving "repeated measures" factors reported above (and in subsequent sections) have been subjected to the Greenhouse-Geisser correction; hence, the fractional degrees of freedom values reported for the F-tests of such effects.

\section{Sign Legibility Distance}

The minimum size needed by the observers to meet the visibility criterion for each sign was converted to a legibility distance index using the formula: Legibility distance (in meters) = (Regulation Sign Size/Thresholder Size) x Test Viewing Distance. Average legibility distances for each sign as a function of age are enumerated in table 54.

The legibility distance data were analyzed using an Age (3) by Sign (18) ANOVA. Both the main effects of Age $[\mathrm{F}(2,36)=33.7, \mathrm{p}<0.001]$ and $\operatorname{Sign}[\mathrm{F}(4.5,161.5)=304.9, \mathrm{p}<$ $0.0001]$, as well as the Age by Sign interaction $[\mathrm{F}(8.9,161.5)=14.5, \mathrm{p}<0.0001]$, were highly significant. Simple-effects analyses revealed that the Sign factor was highly significant for all three age groups: Young $[\mathrm{F}(4.5,161.5)=148.4, \mathrm{p}<0.0001]$, Middle-aged $[\mathrm{F}(4.5,161.5)=$ 126.7, $\mathrm{p}<0.0001]$, and Older $[\mathrm{F}(4.5,161.5)=43.8, \mathrm{p}<0.001]$. Similar results were obtained for the simple-effects analyses of the Age factor. Highly significant age effects $(\mathrm{p}<0.01)$ were obtained for all 18 signs examined. Simple-simple age effects analyses were performed in order to isolate the specific nature of the age-group effect (i.e., young vs. middle-aged vs. older) for each of the 18 test signs. The results of these statistical comparisons are summarized in the column labeled "Age Diff' in table 54. Significant ( $p<0.001)$ young vs. older group differences emerged for all signs. Similarly, significant middle-aged vs. older group differences emerged for all signs except the PAVEMENT ENDS and SCHOOL BUS STOP AHEAD signs. However, the young vs. middle-aged group contrasts yielded no significant age differences for any of the 18 signs. For all three types of signs (Modified, Redesigned, and Novel), legibility distance was closely related to driver age, with the mean distance for the older group being about $2 / 3$ that for the young group (see figure 29). 
Table 54. Highway signs ranked on legibility distance (m) for low luminance condition: nighttime-with-glare legibility (phase 2).

$\underline{\text { Modified Signs }}$

\begin{tabular}{|c|c|c|c|c|c|c|c|}
\hline $\begin{array}{c}\text { USD } \\
\text { Rank } \S\end{array}$ & $\begin{array}{c}\mathrm{UC} \\
\text { Rank }\end{array}$ & $\begin{array}{c}\text { Sign } \\
\text { Description } \\
\end{array}$ & $\begin{array}{l}\text { Age } \\
\text { Diff }\end{array}$ & Young & $\begin{array}{l}\text { Middle- } \\
\text { Aged }\end{array}$ & $\underline{\text { Older }}$ & $\begin{array}{l}\text { All } \\
\text { Groups } \\
\end{array}$ \\
\hline 1 & 1 & $\begin{array}{l}\text { Cross Road } \\
\text { (W2-1) }\end{array}$ & $\mathrm{ac}$ & $\begin{array}{l}366.9 \\
(80.5)\end{array}$ & $\begin{array}{l}341.9 \\
(53.7)\end{array}$ & $\begin{array}{l}192.4 \\
(50.1)\end{array}$ & $\begin{array}{c}292.1 \\
(100.5)\end{array}$ \\
\hline 2 & 2 & $\begin{array}{l}\text { Right Curve } \\
\text { (W1-2R) }\end{array}$ & $\mathrm{ac}$ & $\begin{array}{l}305.7 \\
(91.1)\end{array}$ & $\begin{array}{l}284.9 \\
(55.5)\end{array}$ & $\begin{array}{l}148.6 \\
(42.9)\end{array}$ & $\begin{array}{l}238.9 \\
(91.1)\end{array}$ \\
\hline 3 & 4 & $\begin{array}{l}\text { Divided Hwy Ends } \\
\text { (W6-2) }\end{array}$ & $\mathrm{ac}$ & $\begin{array}{l}242.8 \\
(80.3)\end{array}$ & $\begin{array}{l}179.6 \\
(44.9)\end{array}$ & $\begin{array}{c}96.1 \\
(30.8)\end{array}$ & $\begin{array}{l}166.9 \\
(80.3)\end{array}$ \\
\hline 4 & 3 & $\begin{array}{l}\text { Hospital } \\
\text { (D9-2) }\end{array}$ & $\mathrm{ac}$ & $\begin{array}{l}203.7 \\
(67.5)\end{array}$ & $\begin{array}{l}182.6 \\
(43.7)\end{array}$ & $\begin{array}{l}100.7 \\
(39.5)\end{array}$ & $\begin{array}{l}157.6 \\
(67.8)\end{array}$ \\
\hline 6 & 5 & $\begin{array}{l}\text { Keep Right } \\
\text { (R4-7) }\end{array}$ & $\mathrm{ac}$ & $\begin{array}{l}184.1 \\
(49.8)\end{array}$ & $\begin{array}{l}136.8 \\
(37.3)\end{array}$ & $\begin{array}{c}79.6 \\
(31.0)\end{array}$ & $\begin{array}{l}129.4 \\
(58.5)\end{array}$ \\
\hline \multirow[t]{2}{*}{7} & 8 & $\begin{array}{l}\text { No Parking } \\
\text { (R8-3a) }\end{array}$ & $\mathrm{ac}$ & $\begin{array}{l}126.4 \\
(32.4)\end{array}$ & $\begin{array}{l}100.6 \\
(27.2)\end{array}$ & $\begin{array}{c}56.0 \\
(16.2)\end{array}$ & $\begin{array}{c}91.4 \\
(39.1)\end{array}$ \\
\hline & & Mean & & 238.3 & 204.4 & 112.2 & 179.4 \\
\hline
\end{tabular}

Notes:

USD Rank - Ordinal ranking of signs based upon the performance of the young group

UC Rank - Ordinal ranking of the same signs using Daytime Visibility data collected at the University of Calgary

Age Diff (i.e. post hoc comparison) Legend:

a Young $>$ Older $(p<0.001)$

b Young $>$ Middle-aged $(p<0.001)$

c Middle-aged $>$ Older $(p<0.001)$

$\S$ Spearman Rank-Order Correlation (Rho) analysis of the Calgary daytime versus

USD nighttime visibility data across signs yielded a value of $0.965(p<0.0001)$.

( ) Standard deviations appear in parentheses. 
Table 54. Highway signs ranked on legibility distance $(m)$ for low luminance condition: nighttime-with-glare legibility (phase 2) (continued).

\section{$\underline{\text { Redesigned Signs }}$}

\begin{tabular}{|c|c|c|c|c|c|c|c|}
\hline $\begin{array}{c}\text { USD } \\
\text { Rank } \S\end{array}$ & $\begin{array}{c}\mathrm{UC} \\
\mathrm{Rank} \\
\end{array}$ & $\begin{array}{c}\text { Sign } \\
\text { Description }\end{array}$ & $\begin{array}{l}\text { Age } \\
\text { Diff }\end{array}$ & $\underline{\text { Young }}$ & $\begin{array}{l}\text { Middle- } \\
\text { Aged }\end{array}$ & $\underline{\text { Older }}$ & $\begin{array}{l}\text { All } \\
\text { Groups }\end{array}$ \\
\hline 5 & 6 & $\begin{array}{l}\text { Advance Flagger } \\
(W 20-7 a)\end{array}$ & $\mathrm{r}$ ac & $\begin{array}{l}162.1 \\
(79.5)\end{array}$ & $\begin{array}{l}159.5 \\
(46.1)\end{array}$ & $\begin{array}{c}84.8 \\
(22.6)\end{array}$ & $\begin{array}{l}131.6 \\
(63.6)\end{array}$ \\
\hline 11 & 13 & $\begin{array}{l}\text { Pavement Ends } \\
\text { (W8-3a) }\end{array}$ & $\mathrm{a}$ & $\begin{array}{l}77.6 \\
(21.4)\end{array}$ & $\begin{array}{l}69.2 \\
(9.2)\end{array}$ & $\begin{array}{c}52.3 \\
(13.7)\end{array}$ & $\begin{array}{c}65.3 \\
(19.9)\end{array}$ \\
\hline 13 & 14 & $\begin{array}{l}\text { Bicycle Crossing } \\
(\mathrm{W} 11-1)\end{array}$ & $\mathrm{ac}$ & $\begin{array}{c}73.2 \\
(17.6)\end{array}$ & $\begin{array}{c}64.8 \\
(15.0)\end{array}$ & $\begin{array}{l}41.7 \\
(4.6)\end{array}$ & $\begin{array}{c}58.5 \\
(18.9)\end{array}$ \\
\hline 16 & 17 & $\begin{array}{l}\text { Ranger Station } \\
(\mathrm{RG}-170)\end{array}$ & $\mathrm{ac}$ & $\begin{array}{c}57.7 \\
(13.8)\end{array}$ & $\begin{array}{c}56.8 \\
(12.0)\end{array}$ & $\begin{array}{c}31.4 \\
(10.2)\end{array}$ & $\begin{array}{c}47.3 \\
(17.5)\end{array}$ \\
\hline 17 & 15 & $\begin{array}{l}\text { Campfire } \\
(\mathrm{RA}-030)\end{array}$ & $\mathrm{ac}$ & $\begin{array}{c}62.1 \\
(21.5)\end{array}$ & $\begin{array}{c}50.8 \\
(14.5)\end{array}$ & $\begin{array}{l}25.9 \\
(7.3)\end{array}$ & $\begin{array}{c}44.7 \\
(21.4)\end{array}$ \\
\hline 18 & 18 & $\begin{array}{l}\text { Mandatorv Seatbelt } \\
(\mathrm{R} 16-1)\end{array}$ & $\mathrm{ac}$ & $\begin{array}{l}58.6 \\
(26.2)\end{array}$ & $\begin{array}{c}51.0 \\
(14.6)\end{array}$ & $\begin{array}{l}23.9 \\
(9.0)\end{array}$ & $\begin{array}{c}42.9 \\
(23.0)\end{array}$ \\
\hline & & Mean & & 81.9 & 75.4 & 43.3 & 58.4 \\
\hline & & & Nove & & & & \\
\hline $\begin{array}{l}\text { USD } \\
\text { Rank } \S\end{array}$ & $\begin{array}{c}\mathrm{UC} \\
\mathrm{Rank} \\
\end{array}$ & $\begin{array}{c}\text { Sign } \\
\text { Description } \\
\end{array}$ & $\begin{array}{l}\text { Age } \\
\text { Diff }\end{array}$ & $\underline{\text { Young }}$ & $\begin{array}{l}\text { Middle- } \\
\text { Aged }\end{array}$ & $\underline{\text { Older }}$ & $\begin{array}{l}\text { All } \\
\text { Groups }\end{array}$ \\
\hline 8 & 10 & $\begin{array}{l}\text { Horse Drawn } \\
\text { Vehicle }\end{array}$ & ac & $\begin{array}{l}104.7 \\
(24.8)\end{array}$ & $\begin{array}{c}98.6 \\
(26.9)\end{array}$ & $\begin{array}{c}50.8 \\
(10.9)\end{array}$ & $\begin{array}{c}82.1 \\
(32.6)\end{array}$ \\
\hline 9 & 9 & Cross Winds & $\mathrm{ac}$ & $\begin{array}{l}100.0 \\
(27.5)\end{array}$ & $\begin{array}{c}87.9 \\
(20.9)\end{array}$ & $\begin{array}{l}47.5 \\
(6.7)\end{array}$ & $\begin{array}{c}76.1 \\
(30.1)\end{array}$ \\
\hline 10 & 7 & Lane Reduction & ac & $\begin{array}{c}92.5 \\
(27.1)\end{array}$ & $\begin{array}{c}72.7 \\
(13.2)\end{array}$ & $\begin{array}{l}54.9 \\
(8.3)\end{array}$ & $\begin{array}{c}71.9 \\
(23.2)\end{array}$ \\
\hline 12 & 11 & Fog & $\mathrm{ac}$ & $\begin{array}{c}83.7 \\
(25.9)\end{array}$ & $\begin{array}{l}70.8 \\
(21.1)\end{array}$ & $\begin{array}{l}45.5 \\
(8.6)\end{array}$ & $\begin{array}{c}65.0 \\
(24.9)\end{array}$ \\
\hline 14 & 12 & Truck Entrance & $\mathrm{ac}$ & $\begin{array}{c}74.0 \\
(30.5)\end{array}$ & $\begin{array}{c}54.5 \\
(10.8)\end{array}$ & $\begin{array}{l}38.7 \\
(4.2)\end{array}$ & $\begin{array}{c}54.4 \\
(22.9)\end{array}$ \\
\hline \multirow[t]{2}{*}{15} & 16 & $\begin{array}{l}\text { School Bus Stop } \\
\text { Ahead }\end{array}$ & $\mathrm{a}$ & $\begin{array}{c}76.6 \\
(26.5)\end{array}$ & $\begin{array}{c}54.8 \\
(11.6)\end{array}$ & $\begin{array}{l}45.5 \\
(2.7)\end{array}$ & $\begin{array}{c}57.9 \\
(20.4)\end{array}$ \\
\hline & & Mean & & 88.6 & 73.2 & 47.2 & 67.9 \\
\hline
\end{tabular}


The legibility distance data collected under conditions of glare in the present study were compared to similar measures observed in a companion phase 2 study (viz., Nighttime Visibility without Glare). A visibility reducing effect of glare appears to emerge in the data of the older group. Statistical analyses revealed no significant effect of glare on sign visibility for either the young or middle-aged groups. However, highly significant glare $[\mathrm{F}(1,32)=16.2$, $\mathrm{p}<0.0003]$ and glare by sign interaction $[\mathrm{F}(4,130.6)=5.23$, $\mathrm{p}<0.0006]$ effects were obtained in the comparison of the older group.

\section{Predictors Of Sign Legibility}

Photopic acuity measures and complete contrast sensitivity functions (CSF's) collected under low photopic luminance conditions with, and without the presence of intense peripheral glare, were collected from all of the subjects participating in the current study. Preliminary correlational analyses were performed in order to assess the degree to which variations in visual ability, as indexed by acuity and the CSF, might be associated with concomitant variations in overall highway sign visibility performance. In order to simplify the analyses based upon contrast sensitivity, the CSF functions obtained from each subject were fitted with a 3rd order polynomial, which was used to parameterize the CSF's into two descriptive indices: the highest contrast sensitivity value observed ("peak CS"), and the half-amplitude high spatial frequency cutoff ("high cutoff'). The mean values observed in each age group for each of these CSF parameters, as well as photopic visual acuity, are presented in table 53. Pearson product-moment correlations were calculated between average legibility distance (collapsed across all signs) and each of the following measures of basic visual function: acuity, log peak CS (glare-absent), high spatial frequency cutoff (glare-absent), log peak CS (glare-present) and high spatial frequency cutoff (glare-present). The resulting correlation coefficients are summarized in table 55.

Table 55. Mean overall sign legibility distance correlational analyses: nighttime-with-glare legibility distance (phase 2).

(all subjects: $\mathrm{N}=40$ )

\begin{tabular}{|c|c|c|}
\hline \multicolumn{3}{|c|}{$($ all subjects: $\mathrm{N}=40)$} \\
\hline & $\frac{\text { Correlation }}{\text { coefficient }}$ & value \\
\hline Age & -0.810 & $<0.001$ \\
\hline Acuity (minarc) & -0.396 & $<0.02$ \\
\hline $\begin{array}{l}\text { Log peak contrast sensitivity } \\
\text { (glare-absent) }\end{array}$ & 0.397 & $<0.02$ \\
\hline $\begin{array}{l}\text { High spatial frequency cutoff } \\
\text { (glare-absent) }\end{array}$ & 0.428 & $<0.01$ \\
\hline $\begin{array}{l}\text { Log peak contrast sensitivity } \\
\text { (glare-present) }\end{array}$ & 0.765 & $<0.001$ \\
\hline $\begin{array}{l}\text { High spatial frequency cutoff } \\
\text { (glare-present) }\end{array}$ & 0.515 & $<0.01$ \\
\hline
\end{tabular}


For the overall sample, adult age had the highest correlation with average sign legibility distance $(r=-0.810)$. Legibility distance decreased as age increased. Although not as strongly correlated as the age factor, acuity $(\mathrm{r}=-0.396)$, as well as glare-absent log peak CS $(\mathrm{r}=0.397)$ and high spatial frequency cutoff of the CSF $(r=0.428)$, were significantly (but weakly) related to overall sign visibility. Increases in the minimum angle of spatial resolution (in minarc) were associated with decreases in sign legibility distance, while increases in peak CS and the cutoff spatial frequency were associated with improvements in the maximum legibility distance. Interestingly, the CSF parameters yielded under the glare-present condition were found to be much more strongly related to sign legibility distance (glare-present log peak CS $(r=0.765)$ and high spatial frequency cutoff $(r=0.515)$ ).

\section{DISCUSSION}

The strong and systematic effects of age and signs observed previously under daytime and nighttime viewing conditions were replicated under the nighttime-with-glare condition imposed in the current study (see table 54). The introduction of the glare source, however, exerted no systematic effects upon maximum sign legibility distance among young and middle-aged observers. Yet, a systematic and statistically significant reduction of sign visibility was observed among the older observers. Hence, the transition from the use of a "static" glare source to a "transient" one appears to have been efficacious. While the impact of glare appeared to be greatest for the CROSS ROAD sign, especially for the older drivers, the relative difference between the two conditions was very similar (approximately 28 percent) across signs.

The magnitude of the glare effects demonstrated by the older driver were both small and "relatively" constant across the diverse subset of signs studied. In addition, the introduction of the glare source had no impact upon the rank-ordering of the sign set on the basis of legibility (relative to both the daytime and nighttime conditions observed previously). This pattern of findings suggests that age differences in the deleterious effects of glare can be generalized across the range of stimuli examined. Hence, the effects of glare upon sign visibility may be modeled through the use of a simple age-weighted "reduction of legibility" constant-greatly simplifying subsequent development of a comprehensive model and optimization guidelines.

The effects of glare upon the contrast sensitivity function were straight-forward: glare reduced sensitivity for all observers at all spatial frequencies, and the magnitude of this glare effect was significantly elevated among the older observers. The previously reported age by spatial frequency interaction for the deleterious effects of glare was not replicated. This is the second time that we have failed to replicate that interaction, strongly suggesting that the previous finding is restricted to special stimulus conditions and is not otherwise generalizable, a conclusion which greatly simplifies future model development efforts.

The power of the contrast sensitivity parameters to predict legibility distance was improved when the CSF was collected in the presence of an intense glare source. In fact, unlike the previous phase 2 daytime and nighttime legibility studies, log peak contrast sensitivity was found to be a much stronger predictor of sign legibility in the presence of glare than was photopic acuity. This is a very informative finding and is currently being studied further. 


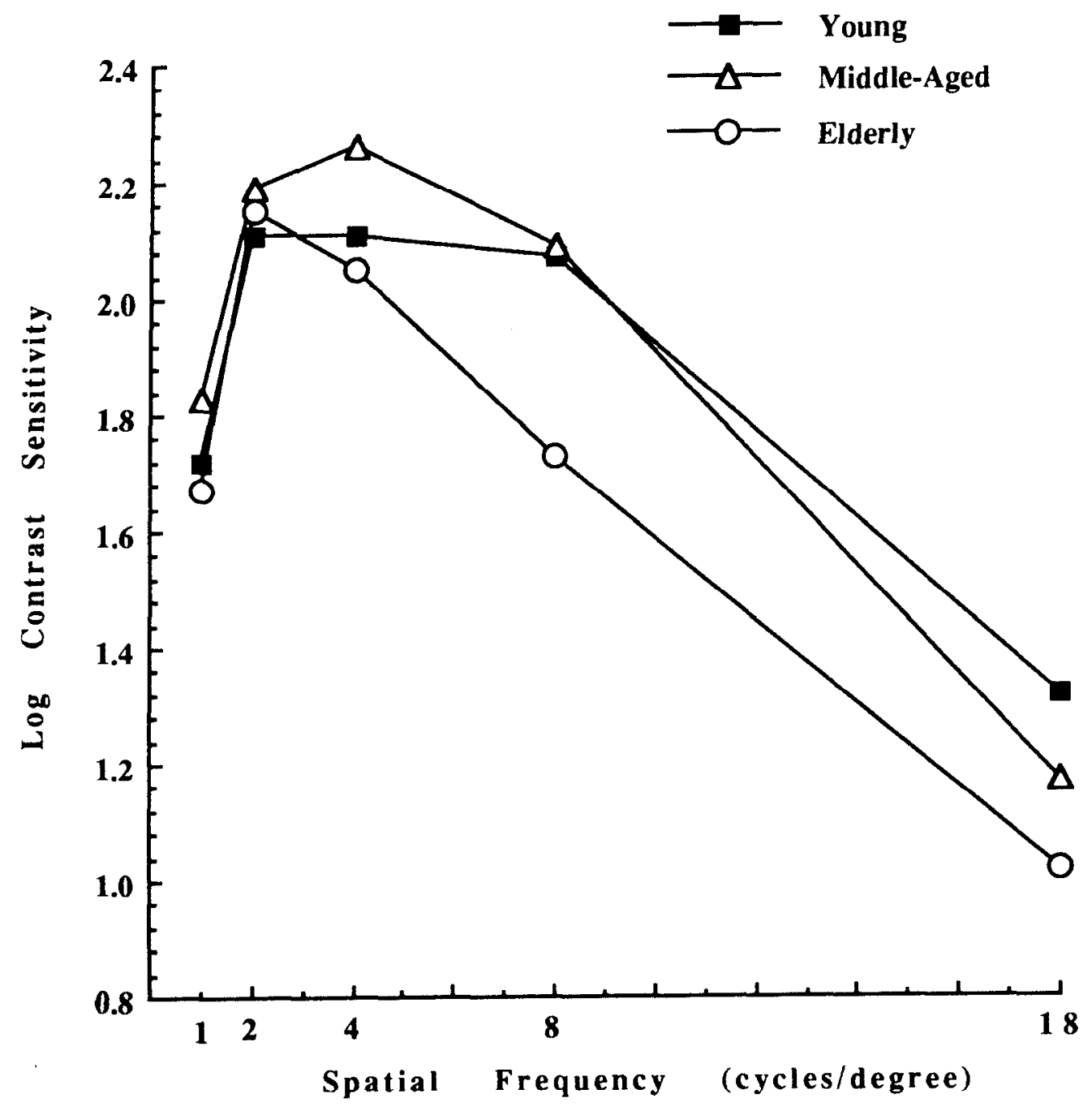

Figure 27. Contrast sensitivity for young, middle-aged, and elderly observers: glare absent condition: nighttime-with-glare (phase 2). 


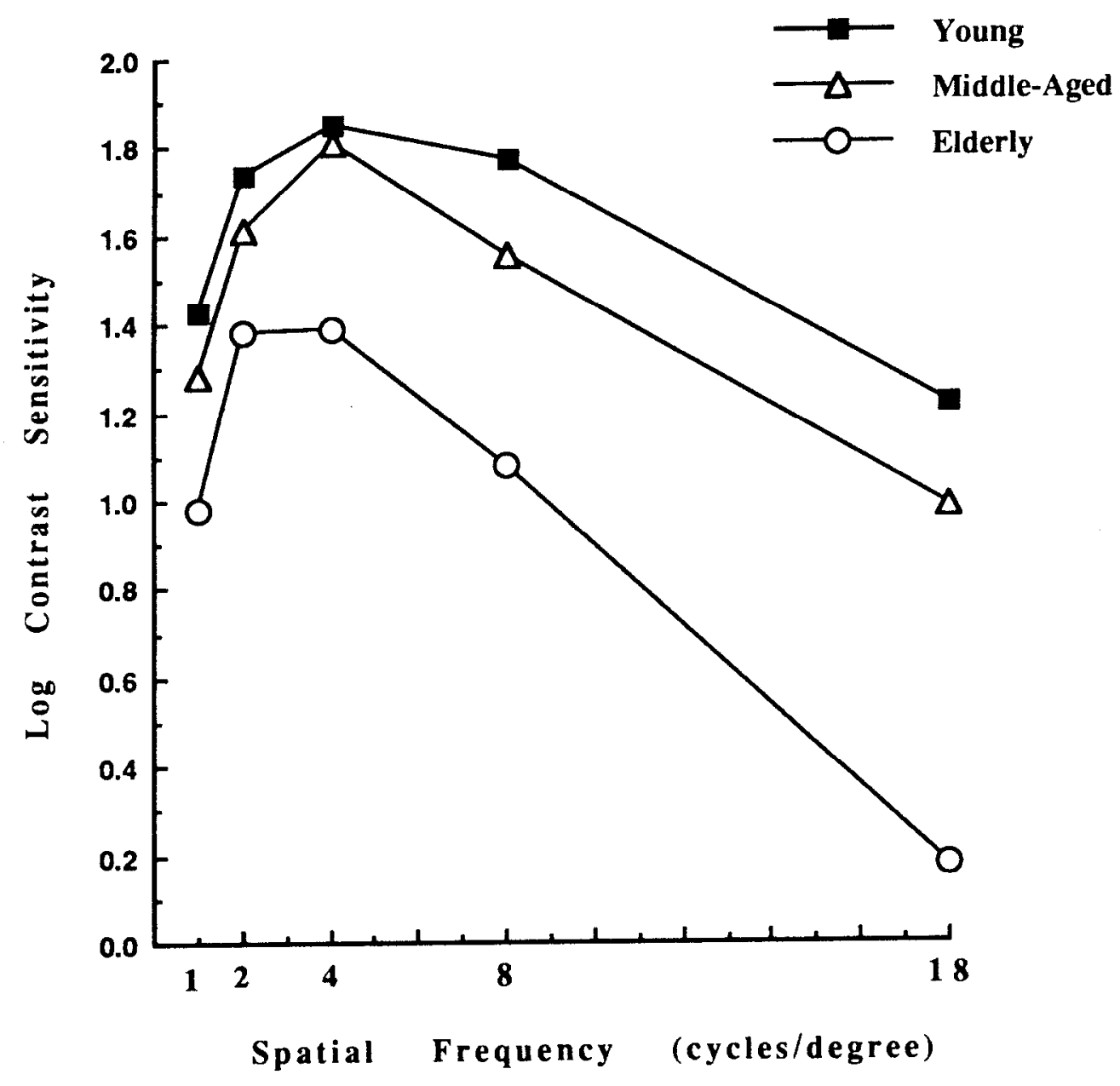

Figure 28. Contrast sensivitity for young, middle-aged, and elderly observers: glare-present condition nighttime-with-glare (phase 2). 


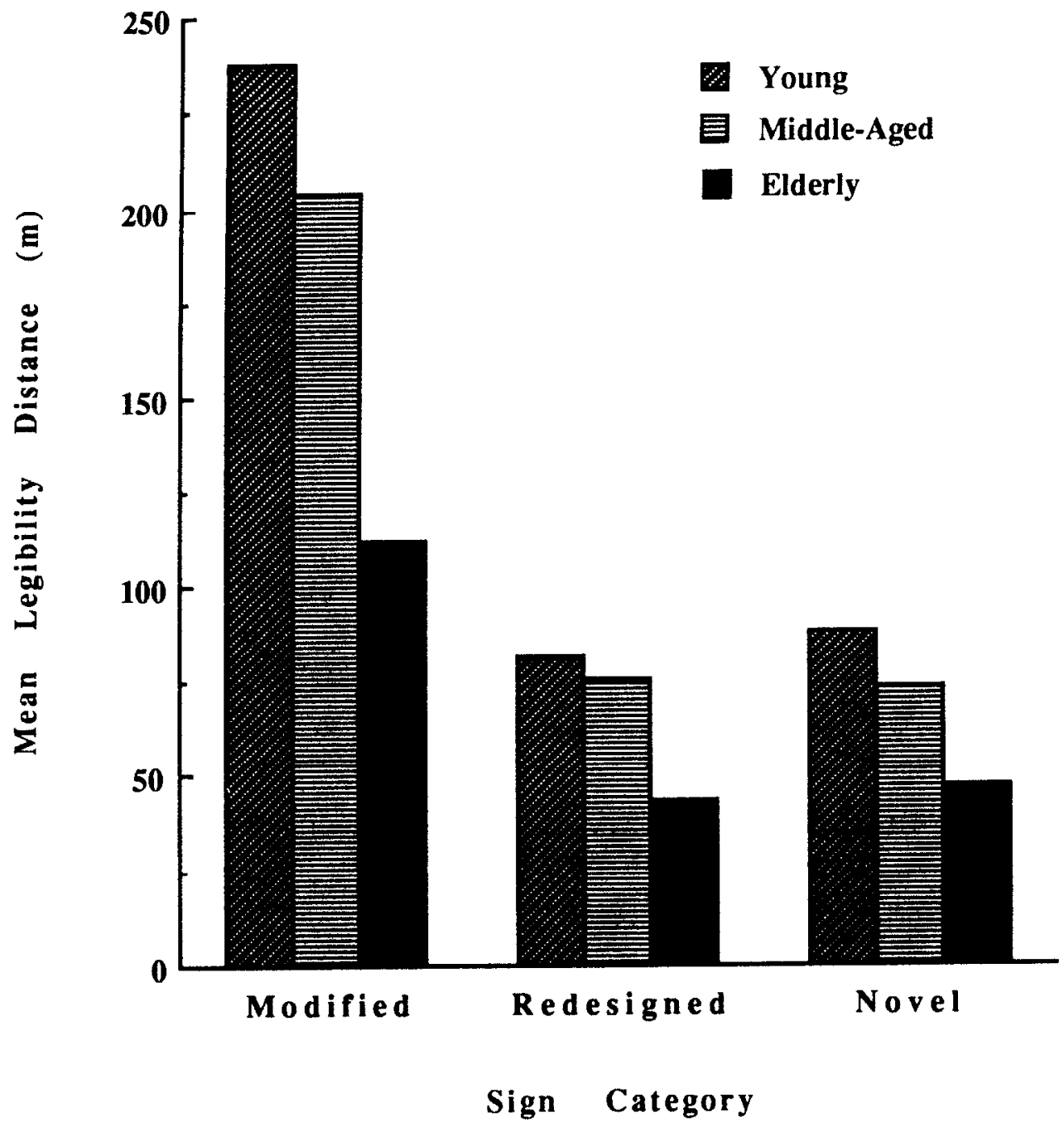

Figure 29. Mean nighttime legibility distances of different sign categories as a function of driver age: nighttime-with-glare (phase 2). 
A control study was conducted which compared legibility distance obtained under static vs. dynamic (flashing on-and-off) viewing conditions. Legibility distance data were collected from 9 older subjects using four highway signs: FLAGGER, PAVEMENT ENDS, RANGER STATION and RIGHT CURVE. All other conditions were identical to those employed in the phase 2 Nighttime Legibility Study. Compared to older subjects examined under static viewing conditions, no significant differences in legibility distance emerged following the implementation of the dynamic viewing procedure. That is, neither the main effect of "presentation mode" $[\mathrm{F}(1,16)=1.04, \mathrm{p}<0.32]$, nor its interaction with the "signs" effect $[\mathrm{F}(1.6,25.7)=1.30$, $\mathrm{p}<0.283$, approached statistical significance. The results of this control study indicate that the decrement in legibility distance observed for older subjects in the phase 2 Glare Study was clearly a manifestation of the glare manipulation, rather than the covarying introduction of the dynamic stimulus presentation mode. 


\section{CHAPTER 14: RECOMMENDED CHANGES TO STANDARD SYMBOL SIGNS AND SYMBOL SIGN DESIGN GUIDELINES}

\section{RECOMMENDED CHANGES TO STANDARD SYMBOL SIGNS}

A major objective of this contract is to offer recommendations for improvements to both individuals and classes of standard symbol signs based on the findings of previous tasks. Although the data from these studies suggest that some improvement could be made in virtually all of the signs studied, in our view a more reasonable and cost-effective approach is to identify those symbols most in need of improvement, and which are thus most likely to benefit from modification or redesign. There are a number of criteria by which a sign's need for improvement can be assessed:

- Comprehension. The message must be readily understandable, or the user will not know whether or how to respond to it. If the meaning of the message is not immediately understood driver error or delay can result.

- Legibility Distance. The critical features of a symbol sign must be legible from as far away as possible so that drivers have time to take the necessary action.

- Glance Legibility. A symbol should be legible when seen for a very brief time. When in heavy high-speed traffic and/or in unfamiliar settings, drivers often have little time to extract information needed from signs. Additionally, at busy urban locations, signs may be seen only briefly when they are obscured by objects such as large vehicles, trees or other signs.

- Reaction Time. For a sign to be effective its message must be understood quickly. Drivers often have only a second or two to interpret and respond to it.

- Conspicuity. A sign must command attention or be easily detected by the person who needs the information.

- Learnability. Symbol messages must be readily understood, or at least easily learned and remembered.

- Relevance. The information on the sign should be easily ignored if it is irrelevant for the driver.

- $\underline{\text { Clarity. }}$ The action to be taken in response to the message must be immediately obvious.

- Distinctiveness. Symbol messages should be easily distinguished from other symbols in the system.

- Uniformity. Signs must share the basic characteristics of other signs of similar type (e.g., color and shape) and be consistent in design from one locale to another.

Some of these criteria (e.g., uniformity and distinctiveness) are in part a function of a sign's class, while others (e.g., conspicuity and relevance) are influenced by contextual variables beyond the sign itself. Emphasis is placed on the two most fundamental measures of an individual sign's effectiveness: comprehension and legibility distance. Particular concern is paid to symbols that are poorly understood and/or which are legible only at relatively short distances. That these two 
measures are not well correlated with one another suggests that they are relatively independent measures of sign effectiveness, and that revisions to signs must consider both dimensions equally. General recommendations regarding common symbol elements are made, followed by recommendations for changes to enhance symbol comprehension and legibility distance of specific signs.

\section{Consistency In Design Of Common Elements}

There are several inconsistencies in the current sign system with respect to the depiction of certain features. Warning signs indicating hazards associated with other "vehicles" on the road are inconsistent with respect to the use of a human figure on/in the "vehicle." For example, a human figure is included in the TRACTOR warning but not in BICYCLE CROSSING. The human figure seems to be an unnecessary detail in warning signs, as the important factor is a clear indication of the type of "vehicle" that presents a hazard on or crossing the roadway. It could be argued, however, that the human figure is more important in recreation symbols, to indicate an activity, as opposed to a hazard. Such figures appear in the CANOEING, SNOWMOBILING, and SLEDDING signs, but they are not depicted with a consistent design.

\section{RECOMMENDATIONS TO ENHANCE THE COMPREHENSION OF STANDARD SYMBOL SIGNS}

Improvements are suggested for signs based on mean percent correct comprehension and mean day legibility distance of the 85 symbols tested in the initial phase of the study. Specifically, the following criteria were used for selecting symbols for recommended changes:

- Comprehension: mean comprehension levels of less than 50 percent.

- Legibility distance: mean day legibility distances in the lowest quartile of all signs

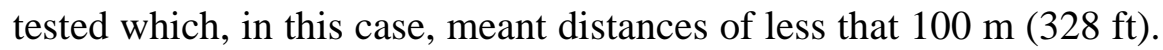

Although somewhat arbitrary, these criteria seem appropriate in view of the expected performance of traffic sign symbols on modern highways. It should be noted that the relative importance of these two criteria depend somewhat on the specific message, as well as the consequences for drivers when they don't understand the symbol or are unable to identify it at the appropriate distance. For example, legibility distance is less important for signs intended for pedestrians (e.g., NO HITCHHIKING) or slow moving vehicles (e.g., NO PARKING), than for warning signs, which are typically viewed at highway speeds. Understanding of recreational symbols may be more important than legibility distance, yet the level of comprehension is less important than is that of regulatory signs (e.g., NO LEFT TURN).

The format for presenting the proposed comprehension changes to signs is:

- Sign name (MUTCD Number; and mean percent correct comprehension)

- Problem with the standard design

- Recommended change to the design. 


\title{
Recommended Changes To Regulatory Signs
}

\author{
1. Straight or Left (R3-6; 16.0 percent)
}

This lane control message was confused with the intersection control sign. The difference is that this message is intended to indicate permissible vehicle movements from the lane over which it is placed, while an intersection control sign relates to maneuvers for the intersection as a whole. Some drivers thought that it meant no "right turn," while others said "left or straight." The scoring criteria for this sign were stringent, requiring mention that the message applied to the specific lane. Addition of the word ONLY, as with R3-5, should be considered. It is likely that this sign would be better understood in its real-world context. It may be necessary to conduct a public education campaign to reduce this misunderstanding.

\section{Recommended Changes To Warning Signs}

\section{Added Lane (W4-3; 25.5 percent)}

This sign was often confused with the merge sign. Responses such as "merge" and "merging traffic from the right" were common. While this difference may seem subtle, the consequences of slowing to merge or yield when it is unnecessary and undesirable to do so are to increase the probability of a rear-end collision and to delay traffic flow by slowing when other drivers behind you are expecting you to maintain or even increase speed when entering a roadway with an added lane. Many thought it meant lane ends. Public education may be needed to address this problem.

\section{Double Arrow (W12-1;34.8 percent)}

Difficulties with this sign appear to be related to its inappropriate content. The symbol does not suggest to the viewer the concept that traffic can pass on either side of an obstruction. Common errors were "two-way traffic" and "divided road." The scoring criteria on this sign were fairly lenient, with answers such as "two-lane split" and "go left or right" being scored as partially correct. Some subjects indicated that the arrows should be pointed the other way (up at an angle, instead of down), to indicate the direction of traffic flow. This is a logical alternative, since all other symbols with arrows indicating traffic directions (e.g., Curve, Divided Highway) follow this format. It is recommended that this symbol be redesigned, possibly with a concept similar to that used in Canada, which resembles the divided highway symbol, but with a single up arrow splitting to go around the median - WA-17 in the Canadian MUTCD.

\section{Chevron Alignment (W1-8; 36.8 percent)}

Subjects often had the correct general idea, but did not understand that it indicated a sharp curve. However, this sign is likely to be well understood in the roadway context, especially as the sign never appears alone, but typically in a series of signs ranging from 3 to 10 or more. No specific change is recommended for this sign. 
The most common error in response to this symbol was that drivers (28.4 percent) confused it with road narrows, not appreciating the fact that the right lane would end. There is a separate (word) sign for "Road Narrows" and its message does not imply the ending of a travel lane. A problem arises with this confusion, as failure to understand that one's lane ends could lead to conflicts as vehicles attempt to merge at the last moment. This symbol has been redesigned.

\section{Large Arrow (W1-6; 38.9 percent)}

This sign was taken by many drivers to mean "one-way road" or "right turn only." While these are not serious errors, as the concept of going right is there, these responses are not quite correct. However, as with the chevron sign (for which the large arrow may be used in many instances), this message would likely be understood well on the roadway. No change is recommended for this sign.

\section{Slippery \#en Wet (WS-5; 44.6 percent)}

Many drivers missed the concept of wet road surface, thinking that the sign meant slippery road. The problem with this interpretation is that drivers may suddenly slow when it is not necessary or may be even dangerous to do so (e.g., in heavy traffic), causing rear-end collisions. Some indication of a winding or rough road was an element in many of the wrong responses. Possible solutions to this difficulty are to redesign the symbol to depict better the concept of wet surface or to conduct an education campaign.

\section{Bicycle Crossing (W11-1; 44.8 percent)}

This symbol was frequently thought to mean "bicycle path" suggesting that drivers did not understand that it indicates a potential hazard from bicycles crossing the roadway ahead of them. The scoring on this sign was lenient, with common answers such as "watch for bicycles on the road" being accepted. This sign was modified to depict more effectively the idea of a crossing.

\section{Recommended Changes To Guide Signs}

\section{Winter Recreation (I-10; 11.0 percent $)$}

A large proportion of drivers had no idea what this symbol meant, as indicated by the number of no-response and "Don't Know" replies, even though subjects were encouraged to guess if they did not know a sign's meaning. The great amount of small detail in this symbol made the content difficult to interpret. Many drivers responded that the message related to winter, but most thought it meant "snow," "snow on the road," or "slippery road surface." It is recommended that this symbol be deleted from the MUTCD. The message appears to be unnecessary, in view of the fact that there is no similar message for summer recreation, that there are many recreational activities that fall under the heading of "winter recreation" and that the message does not indicate to the viewer which of these are available. As there are specific symbols for the major winter recreations (skiing, skating, sledding, etc.) it would be best to use these where relevant. However, if such a 
message was deemed essential, it could be conveyed with a "set" of symbols in the same way as is done with motorist services signs where they (e.g., food, fuel, phone) are combined on the same sign panel.

\section{Bus Station $\quad(I-6 ; 10.4$ percent $)$}

The message was frequently confused with a widely used symbol with a similar meaning-the front view of a bus to indicate "bus stop." In many jurisdictions, there are apparently two very similar symbols indicating two different messages. In addition, the front view was not always understood as a bus, suggesting that it might be better to use a side profile view of the bus, as is done with trucks and cars on many other symbol signs. Consideration should also be given to the addition of a platform or building of some sort, to correspond with the TRAIN STATION symbol and convey the idea of a terminal, as opposed to a stop.

\section{Recommended Changes To Recreational/Cultural Signs}
1. Shelter
(RA-110; 26.8 percent)

The most frequent errors (at least 40 percent) indicated that there was no understanding of the nature of the "accommodation." While most understood the symbol as referring to some sort of accommodation, (e.g., motel, hotel, camping) it was not seen as an outdoor, recreational, type of facility. Changes proposed for this symbol are indicated in the section on legibility distance.

\section{Amphitheater (RL-010; 28.3 percent)}

The small details and inappropriate sign content made this difficult to understand. Common errors indicated the concentric arcs to be understood as waves of electricity, sound, or water. The concentric arcs do not suggest rows of seats, nor does the block on the right resemble a stage. A profile view of seated people and modification of the stage would help.

\section{Recommended Changes To School Signs}

\section{School Advance (S1-1; 41.3 percent)}

The findings in this study reflect those of previous surveys that have shown a confusion between the school advance and the school crossing messages (22.6 percent thought it indicated school zone). Many thought it meant "school crossing," while many others failed to understand that the sign related to school. Another common incorrect response was "pedestrian crossing" indicating a lack of understanding that the pedestrians involved were children. Some subjects thought that figures on the sign depicted adults with briefcases. These errors may not be serious ones, as the correct driving response would be to slow down and watch for children, if it was understood that the pedestrians were children. However, the error of thinking that the "school crossing" was a school advance message could cause problems. It may be worth considering a different color for school signs for easier identification by drivers. 


\section{Driver Education}

One of the remedies for some of the poorly understood symbols is a campaign to educate drivers about the meanings of these messages. Most symbols are introduced into the signing system without any systematic effort to inform licensed drivers of these new messages, except to place an "educational tab" beneath the symbol for a period of time. This tends to be done for regulatory messages. Although this practice undoubtedly enhances understanding of many symbols, it is not the sole answer to driver education about symbols, as evidenced by the poor understanding of many of those tested in this project. This lack of comprehension is especially evident in the case of recreation and motorist services symbols. These, of course, are somewhat less important than warning and regulatory messages, but if they are to be used to convey information to drivers, then their legibility and understandability should be maximized.

\section{RECOMMENDATIONS TO ENHANCE THE LEGIBILITY DISTANCE OF STANDARD SYMBOL SIGNS}

\section{Design Constraints}

Although the legibility distance at which a symbol's features are discernible can be enhanced in several ways, not all of them apply equally well to the redesign or modification of signs that must conform to the context of an extant sign system. We have assumed, for example, that the color, retroreflectivity, shape, placement and size of revised signs must be consistent with others of the same type (e.g., Regulatory, Warning, etc.). This, in turn, means that little change in a sign's contrast level is feasible and that the possibilities for novel spatial layouts are limited. For reasons of comprehension, we have also assumed that symbol signs should convey their messages in a concrete rather than abstract manner. Thus, the recommendations for improvement to current signs that are made in this section are directed at the optimization of the spatial characteristics of graphically realistic displays.

\section{General Changes}

Typically, the legibility distance of a sign is limited by the size, shape and/or location of one or more of the small details that are critical to conveying its message. Where feasible, such details should be eliminated, or otherwise simplified, enlarged, and separated. This is particularly well exemplified by the CROSS ROAD sign, the mean legibility distance of which exceeded that of all other signs tested. The modified version of the CROSS ROAD also manifested the greatest absolute degree of improvement over the standard version. Presumably, the visibility of this sign is a function of its simplicity, size and the spatial separation of its two principal components. In general, the following suggested changes "push" signs in this direction. It should be noted, however, that except where indicated, these recommendations have not been empirically validated. Although our studies show that the legibility distance of both "good" and "bad" signs can be improved, in order to enhance the cost-effectiveness of the process, as already noted, recommendations for change have been offered in order of decreasing visibility for signs in the bottom 25 percent of signs tested (i.e., those with a legibility distance below $100 \mathrm{~m}(328 \mathrm{ft})$ - see table 2, chapter 3, Daytime Legibility of Traffic Sign Symbols). 


\section{Regulatory Signs}

As a class, the average legibility distance of the 11 regulatory signs evaluated was similar to the mean of all signs that were tested. Consistent with this, they were only proportionately represented among the worst signs. The very worst sign tested, however, (NO HITCHHIKING) was also of this type. The format for presenting recommended changes is similar to that used in the section on Comprehension above:

- Sign name (MUTCD \#; mean legibility distance).

- Problem(s) with the standard design.

- Recommendations for change.
1. No Bicycles
(R5-6; $91.8 \mathrm{~m})$

The "bicycle" is difficult to identify because it is composed of several small features. Its visibility would be enhanced if the thickness of the "bicycle frame" was increased significantly, the seat elevated above the frame and separated from it, and by removal of the unnecessary pedal detail. An example of such a bicycle is provided by the modified BICYCLE CROSSING sign (figure 17, chapter 9).

\section{Mandatory Seatbelt}

(R16-1; $75.7 \mathrm{~m})$

The frontal outline view of the "armless driver" is highly stylistic, and the contours that define the driver blend with those of the "belt." This sign was redesigned using a mixed frontal/side solid silhouette "driver" with an open space surrounding an overlapping "belt" (figure 18, chapter 9). As a result, the sign was readily comprehended by almost all drivers. Mean legibility distance of the sign, however, was slightly decreased, suggesting the possible need for a larger Seat belt and open space around it.
3. No Hitchhiking
(R9-4a; $44.4 \mathrm{~m})$

The "fingers" are insufficiently separated for the "hand" to remain identifiable from a distance. The "no-thumbing" message would be more clearly communicated if the fingers were more widely spaced. Additionally, the "right hand pointing to the left" should be changed (i.e., "rotated") to a "left hand pointing right" so that the fingers are no longer obscured by the prohibitive slash. Extension of the forearm might also increase this sign's legibility distance. It should also be recognized that the No Hitchhiking sign is directed primarily at pedestrians; presumably improving its visibility is less critical than it would be for signs directed at motorists.

\section{Warning Signs}

The legibility distance of Warning signs as a class exceeds that of all other sign types. Only two Warning signs were among the 24 low-visibility-distance signs selected for recommended changes. 
The railroad feature is composed of very small details ("ties" and "rails") whichunder blur, or when viewed at a distance, look like a bar or possibly a less distinct parallel roadway. Visibility could be increased by reducing the number of ties, by increasing their size, and by shortening the main road section so that it could be shifted laterally to increase the separation between the "road" and "railroad."

\section{Pavement Ends}

(W8-3a; $93.8 \mathrm{~m})$

The "rough" or "gravel" part of the road ahead is composed of patches so small that they are indistinct from a distance. It is suggested that this sign be revised consistent with the approach employed in this research (see figure 18, chapter 9). It depicts the side profile of a car with its rear tires on a smooth surface, and its front tires above a chunky and undulating surface. Even when distant viewing makes the "roughness" conveyed by the chunks indistinct, this property is still implied by the visible undulations in the road surface.

The revised version of the PAVEMENT ENDS symbol was shown to be slightly more difficult to understand than was the standard version. However, legibility distance was improved by 42.7 percent [from 75 to $107 \mathrm{~m}$ ( 246 to $351 \mathrm{ft}$ )] for the new version. Although suggestions were made by subjects and members of the project team, that the current word message for "rough road" could replace the "pavement ends" symbol, it is important for the driver to understand that this message indicates an undefined distance of unpaved road ahead, not simply a section of rough pavement or a short section of gravel road at a construction site.

\section{Guide Signs}

\section{Recreational Vehicle Sanitary Station (D9-3a; $91.5 \mathrm{~m})$}

This sign's visibility is reduced by several small and sometimes superfluous details (e.g., trailer hitch and support, hubcaps). The "trailer" could be increased in size, including the size and separation of the tire from the trailer. The "ground block" could be reduced in size and a road surface added under the trailer. At a distance the "down arrow" can't be seen; a much broader short arrow entering a much wider channel into the "tank" would help.

\section{Winter Recreation}

(I-10; $90.2 \mathrm{~m})$

The "snowflake" used is too far removed from the general "recreation" message it is intended to convey. It is also too highly detailed. Unless a generic winter sign is necessary, it should be eliminated from the Manual. In its place, a multi-panel sign could be used which presents simple silhouettes illustrating simple recreational activities.
3. Library
$(\mathrm{I}-82 ; 87.7 \mathrm{~m})$

The "person" is insufficiently realistic to be readily identified, the "arm" is indistinct and the "book" is not "open" sufficiently to be seen at a distance. Also, the intended stylized "L" composed by the figure is not discernible as such. A simple but symmetrical profile of a person, a 
clearly separated arm and a more open book might be more effective. The human figure should be consistent in design with those appearing in other signs.
4. Food
(D9-8; $86.4 \mathrm{~m})$

The "knife" and "fork" need to be considerably larger and well-separated from one another. Removal of the one of the tines of the fork, shortening of the handle of the fork, "sharpening" the point of the knife, and increasing the width difference between the knife handle and blade would enhance this sign's visibility.
5. Diesel
(D9-11; $81.2 \mathrm{~m})$

The small tightly looped "hose" on the side of the pump and the letter "D" both limit this sign's visibility. The $\mathrm{D}$ could be increased in size and stroke width, the hose made thicker, moved further off both the pump and ground and its "loop" opened up significantly.
6. Rest Area
(DS-5a; $77.2 \mathrm{~m})$

The "table's" legibility might be increased by simplifying the table by removing the "seat pads" and by making the table "legs" continuous with the table top.

\section{Emergency Medical Service (D9-13;63.0 m)}

Due to the small details used to convey its message, the legibility distance of the EMERGENCY MEDICAL SERVICES symbol was very poor. While the use of a red cross would be an ideal solution, regulations restricting its use apparently prevent this application for traffic signs. The red cross has been used in other parts of the world (e.g., Europe, South America) for this message. Another solution worthy of consideration is a white cross on a blue background. If properly designed (to maintain contrast on a blue rather than a yellow panel), the symbol is likely to be well understood, and certainly will be highly legible at a distance. Variations of a cross, and a cross with an injured hand, have also been used for industrial and public information applications, and may also be worth considering.
8. Train Station
$(\mathrm{I}-7 ; 54.9 \mathrm{~m})$

Numerous details, some of them unnecessary, make this sign one of the worst in the MUTCD. To achieve a distinctive perspective of a train, a "diesel engine" is presented from the front. The tracks upon which the engine rests appear to recede into the distance by means of linear perspective. The impact of 3-D cues on the visibility on symbol is not known, and until that is determined, perhaps they should be avoided. The sign's visibility might be increased by use of a large simple side profile of an engine on a track (perhaps of a steam locomotive with large smokestack such as is used in Europe and parts of Asia), a distinctive large-eave shelter done in line-drawing form and by the elimination of the "passenger."

\section{Recreational/Cultural Signs}

Because the legibility distance of Recreational/Cultural signs is generally well below that of other sign types, they are over represented among the least-visible signs (i.e., 11 of 24). Their 
relatively poor legibility is consistent, however, with the fact that while these signs convey information that is of potential importance to travelers, their message is seldom critical to the driving task itself.
1. Canoeing
(RW-020; $94.3 \mathrm{~m})$

The "paddlers" in the sign are very small, especially their arms, heads and paddles. The "bow paddler" could be eliminated and all the features of the "stem paddler," including the "arms" and "paddle" should be enlarged considerably. The upturn of the bow and stem of the should also be increased to enhance the concept of a canoe.
2. Snowmobiling
(RS-070; $94.3 \mathrm{~m})$

Visibility is limited by both the size of the "driver" and the size and location of the snowmobile's "runners." The driver's "helmet" is overly subtle and should be replaced with a larger "separated head," the "seat back" eliminated, and the runners increased in thickness and moved further below from the snowmobile. This sign's legibility distance probably could be enhanced to an even greater extent by the elimination of the human figure.
3. Campfire
(RW-020; 92.9 m)

When viewed from a distance, the three "flames" are insufficiently separated and the pair of "logs" not readily segregated or identifiable (they look somewhat like a pair of cigarettes). When this sign was redesigned (figure 18, chapter 9) its visibility was increased by adding an internal negative-space (i.e., background color) flame. To enhance comprehension, three pseudo-3D logs were added using a perspective view, although the advisability of such depth cues in symbol signs has not been determined (see also the TRAIN STATION sign above).
4. Amphitheater
(RL-010; $84.7 \mathrm{~m})$

The "rows of seats" blur together at a distance and their meaning is ambiguous. Greater separation of the rows along with a greater degree of curvature so as to increase the "convergence" on the "stage" might reduce this limitation. A potentially more promising possibility would be to use a side profile of tiered rows of seats (2 or 3 ) with a person in each row facing the side profile outline of an open "band shell" type amphitheater. This design concept has been used for building signs.
5. Shelter (Sleeping)
(RA-110; $78.8 \mathrm{~m})$

The "sleeper" is far too small and unrealistic (e.g., overly short legs, no feet) and when it is viewed from a distance, it blurs into the shelter floor. A larger, "floating sleeper" with an identifiable leg and feet contours would help. The floor could be left out to avoid contour interactions between floor and sleeper.
6. Ramp (Launch)
(RW-080; $76.5 \mathrm{~m})$

The "trailer" is both too finely constructed and too close to the "boat" to be seen separately from a distance. The boat and trailer could be increased in size, and the contours that define them 
thickened, the boat and trailer separated, the trailer "hubcap" eliminated and the "ripples in the water" made considerably more prominent.
7. Lighthouse
(RG-120; $74.0 \mathrm{~m})$

The features that mark the "lighthouse" including the "viewing gallery" and "rail," "door" and "roof eaves" need to be increased in size so that they can be seen when viewed from afar. The visibility and/or comprehension value of adding "waves" below the lighthouse and/or the addition of light rays emanating from the top of the tower also deserve consideration.

\section{Showers}

(RA-130; $71.3 \mathrm{~m})$

The "water drops" are overly small and from a distance, make the sign readily interpretable as a "lamp" and "light beams." A side profile of a shower head and simple human figure that is consistent with that in other signs, as well as a few large "streams of water" coming from a large side-profile showerhead, should make the sign both more legible and less ambiguous.

\section{Sledding}

(RS-060; $70.2 \mathrm{~m})$

When seen from far away, the "sled" and "rider" become a single amorphous object. Increased separation between sled and rider, and a significant increase in the thickness of the sled's defining contours, would reduce this problem.

10. Skating

(RS-010; $69.1 \mathrm{~m})$

The "skates" are too small to be seen from far away, making it unclear in what activity the "skater" is engaging (the skates resemble short skis). Suggestions for change include bigger skates, a greater separation between "blade" and "boot" with support posts between them, a more distinctive boot, and the addition of an ice surface well below the skates might enhance this sign's visibility.

\section{Ranger Station}

(RG-170; $66.1 \mathrm{~m})$

This sign is overly detailed making critical features too small to be identified from a distance. To increase the sign's legibility distance, the "ranger's body" was eliminated in favor of a "head" and "hat," the contour thickness of the "station" and "flag" was increased, a line drawing was used to define the building and the "door" was removed (see figure 18, chapter 9).

\section{SYMBOL SIGN DESIGN GUIDELINES}

The objective of this part of the project is to recommend guidelines for symbol sign design. The following recommendations are made on the basis of the findings of the studies conducted for this contract, as well as on the literature on symbol sign design. 


\section{Criteria For Design}

While the general criteria for a good traffic sign are given in previous sections of this contract, it should be pointed out that these do not include the specific "performance criteria" for individual signs or classes of signs. The minimum acceptable level of comprehension or minimum legibility distance for signs is a matter of policy, as opposed to scientific discovery. These issues were examined by Dewar in a survey of traffic sign experts and practicing traffic engineers in Australia, Canada, New Zealand, and the United States. ${ }^{(25)}$ His findings reveal that comprehension, conspicuity and legibility distance are seen to be the most important criteria in symbol design, with reaction time and glance legibility next most important. However, that study provided only an idea of the relative importance of these criteria, but say nothing about absolute levels of performance required for symbols. That is, standards for minimum legibility distance, levels of comprehension, etc. have not been addressed. The present series of studies did not deal with these matters, so there is still the need to make decisions about these criteria.

The relative importance of the two major criteria (comprehension and legibility distance) depend on the specific message and the consequences of failure to understand the symbol or to identify it at the appropriate distance, as noted earlier.

\section{Design Constraints}

Although the legibility distance at which a symbol's features are discernible can be enhanced in several ways, not all of them apply equally well to the redesign or modification of signs that must conform to the context of an extant sign system. This, in turn, means that little change in a sign's contrast level is feasible and that the possibilities for novel spatial layouts are limited. For reasons of comprehension, we have also assumed that symbol signs should convey their messages in a concrete rather than abstract manner. As a general principle, it should be realized that not all messages can be presented in symbol form, so words, or words in combination with symbols, will be necessary for certain messages.

\section{RECOMMENDATIONS TO ENHANCE COMPREHENSION OF SYMBOL SIGNS}

\section{Critical Vs. Educational Features}

There can be considered to be two types of details on traffic sign symbols-those necessary for adequate legibility distance and those desirable for easy understanding of the message. The latter are likely to be more detailed/small and may be illegible at the distance from which the message must be understood, but can be discriminated clearly at a closer distance. These are helpful in promoting driver understanding of the symbol's meaning, even though they may not be visible at the distance required to make an appropriate response to the sign. That is, they have "educational value," but do not contribute to identifying the symbol from afar. Care must be taken, however, to ensure that these small details do not interfere with the visibility of the essential details. 


\section{Symbol Complexity}

As a rule of design of any visual message, the simpler, the better. This requires that the basic design concept involve an easily discriminated, easily understood figure with as few details as possible. An example of a sign that violates this principle is the WINTER RECREATION message- a snowflake with great detail, much more even that required for the depiction of a snowflake, if it were appropriate content for this message.

\section{Depiction Of Human Figures}

Warning signs indicating crossings and other signs with "vehicles" are inconsistent with respect to the use of a human figure on/in the "vehicle." For example, a human figure is an element in the TRACTOR warning but not on the BICYCLE CROSSING. The human figure would seem to be an unnecessary detail in warning signs, as the important factor is a clear indication of the type of "vehicle" that presents a hazard on or crossing the roadway. It could be argued that the human figure was more important in recreation symbols, to indicate an activity, as opposed to a hazard. There is such a figure in the CANOEING, SNOWMOBILING, and SLEDDING signs. However, as indicated earlier, consistency in the design of human figures should be achieved. These designs should be standardized with a single design for each of the main postures - standing, walking, sitting, and lying down.

The following symbols currently in the MUTCD have human figures portrayed with a variety of designs:

MANDATORY SEAT BELTS
TRACTOR
PLAYGROUND
WORKER
RANGER STATION
SHELTER (SLEEPING)
CANOEING
ICE SKATING
SCHOOL ADVANCE
SNOWMOBILING

PEDESTRIAN CROSSING
ADVANCE FLAGGER
HIKING TRAIL
LIBRARY
REST ROOMS
SHOWERS
SWIMMING
SLEDDING
SCHOOL CROSSING

The redesigned MANDATORY SEAT BELTS symbol is an exception to the rule of standard figure design.

\section{Depiction Of Vehicles}

Designs of trucks and automobiles are inconsistent in the MUTCD. The following modifications are recommended for truck and automobile symbols:

- Standardize the side, front and rear view of all trucks and automobiles to the same designs.

- Increase the distance between vehicle body and road surface and make the wheels more prominent. Motor vehicles appear in the following signs (** indicates novel symbols developed for this contract): 
Trucks:

NO TRUCKS

WEIGHT LIMIT

NATIONAL NETWORK ROUTE

NATIONAL NETWORK PROHIBITED

HILL

TRUCK ENTRANCE **

Automobiles:

SLIPPERY WHEN WET

PARK AND RIDE

REDUCED VISIBILITY $* *$

WIND GUSTS **

\section{Use Of Ahead Arrow}

The current application of the "ahead arrow" on warning symbols is inconsistent. It occurs, for example, on STOP AHEAD and YIELD AHEAD, but not on SIGNALS AHEAD. The question arises as to when such an arrow is appropriate, since all hazards are ahead of the sign's location. It is suggested that when a specific distance is relevant (e.g., to a stop sign, school crossing, cross road), an ahead arrow and/or a distance tab is appropriate as an addition to the symbol. The distance indication would likely increase the understanding of "ahead" in cases such as the SCHOOL ADVANCE symbol, which was poorly understood in this study. One reason why the error rate was so much bigger for the SCHOOL ADVANCE, than the SCHOOL CROSSING message, was that the former was often confused with the latter, but not vice versa.

\section{Confusions Within Classes Of Signs}

It should be noted that confusions were common in the cases of certain classes of signs. The most obvious of these were:

TURN/CURVE/WINDING ROAD - the differences among these were often not appreciated by the subjects. TURN and WINDING ROAD were often thought to mean "curve." In other words, the degree of hazard was not clearly understood.

NATIONAL NETWORK/TRUCKS PROHIBITED - the NATIONAL NETWORK and its prohibitive version were generally thought to mean truck route and no trucks allowed, indicating poor understanding that these messages related to specific types of vehicles. However, it is assumed that the truck drivers who need this information will be familiar with the meanings of these symbols.

DIVIDED HIGHWAY BEGINS/ENDS - many subjects did not know the difference between the beginning and ending of the divided highway, a potentially dangerous situation, especially if the divided highway is ending. An education campaign may be necessary to increase understanding of the difference. 
SCHOOL CROSSING and SCHOOL ADVANCE - there was considerable confusion between these two symbols, due likely to the great similarity between them. Drivers appear in this case not to appreciate the difference between an advance warning and the location of the hazard itself.

Potential confusion among messages can be reduced by avoiding the use of symbols that look alike, or possibly by using distinctive color coding.

\section{Uniformity Of Design}

Uniformity of design, both within and between signing systems is an aid to understanding, especially for new symbols. Where appropriate, symbols should be designed with content and design similar to those found to be effective in traffic signing systems in other countries, as well as with those effective symbols used for the same messages in public information systems (e.g., buildings).

The available evidence indicates that years of driving experience contribute to increased understanding of symbol signs only for the first few years, with little gain thereafter. Although older drivers are generally much more experienced than their younger counterparts, their comprehension levels are not greater. Relatedly, when comprehension was assessed in the study of age differences in the day legibility of Modified, Redesigned and Novel signs, it was related to years of driving experience only among young drivers

\section{Standards For Comprehension Levels}

Although the effectiveness of a symbol sign is limited by its ease of comprehension, little attention has been paid to developing "acceptable" comprehension standards. Figures of 70 and 75 percent correct comprehension have been used in some jurisdictions. However, this, or indeed any specific cutoff, is arbitrary. The minimum required level of understanding for a symbol would logically depend on the consequences of failure to understand the message. As a general rule, most warning and regulatory messages would be well understood. Misinterpretation of regulatory signs could lead to violating the law, as well as compromise safety (e.g., NO LEFT TURN). Inadequate warning messages could lead to unsafe driver behavior in many situations (e.g., STOP AHEAD, TWO-WAY TRAFFIC). Some regulatory signs have minimal consequences for safety (e.g. NO PARKING, NO HITCHHIKING). Failure to understand guide and cultural/recreational signs is generally less important, as failure to understand them will likely lead only to drivers becoming lost or confused. However, certain ones (e.g., HOSPITAL, EMERGENCY MEDICAL SERVICES), will be important to some drivers. Therefore, separate standards for comprehension may be necessary for categories of signs, as well as certain symbols within categories.

It is recommended that standards be established for acceptable levels of comprehension for each sign or sign type, recognizing that different categories of messages have differential consequences if they are not understood, and that not all messages in a particular category are equally important. An example of this principle might be to establish a minimum of 80 percent average comprehension for regulatory and warning symbols, with no lower than 70 percent comprehension in any one driver age group (young, middle-aged, and older, as used in this series 
of studies). Similar levels for guide and cultural/recreational symbols could be 75 average, with not less than 65 percent for any one age group.

In addition to the establishment of such standards, it would be appropriate to determine the point at which it is reasonable to expect drivers to understand new or redesigned symbols after they have come into use. It is recommended that an educational tab be placed beneath new symbols for 5 years following their introduction into the MUTCD and also into driver licensing handbooks. The 1988 MUTCD specifies a minimum of 3 years for the supplementary word message. The general need for driver education about highway symbols has been addressed earlier. There is clearly a need for this at the present time for existing symbols, as well as any new ones introduced into the system. An evaluation of comprehension levels after this time could determine whether a symbol met the specified criteria. If not, then an increased educational campaign or symbol redesign would be in order. It should be noted, however, that not all messages can be conveyed with a symbol, and that continued low levels of comprehension, following redesign of symbols and a proper education program, suggests that words should be used for the message. In some cases, it may be appropriate to use an existing symbol that conveys a similar message. For example, the TWO-WAY TRAFFIC symbol could replace the DIVIDED HIGHWAY ENDS symbol, if the latter was not well understood.

\section{RECOMMENDATIONS TO ENHANCE LEGIBILITY DISTANCE OF SYMBOL SIGNS}

\section{Legibility (Legibility Distance)}

A sign's legibility determines the sight time available for a driver to "read" the message and to initiate an appropriate response, and is one of the most important determinants of sign effectiveness. Because legibility is a measurable and highly predictable property of individual signs that is consistent across widely varying conditions, it is highly amenable to a guidelinebased approach to sign design or modification. Typically, the legibility distance of a sign is limited by smallest features that are critical to conveying its message. The major goals of this section are to:

- Note the relationships between legibility and driver age, lighting conditions and spatial vision.

- Present guidelines for optimizing the legibility of individual sign features and minimize contour interactions that reduce overall legibility.

- Describe a new design/modification approach for optimizing sign legibility.

\section{Symbol Details}

It is clear from the various legibility distance experiments in this series of studies that the number, size, and configuration of details on individual symbols were major determinants of the distance from which the symbols could be identified. The criteria used in the legibility distance studies required drivers to see clearly and to identify/describe virtually all details of each symbol. In some cases (e.g., RANGER STATION) this strict definition of "legibility" lead to a relatively short legibility distance. The logic behind the use of this procedure was that the symbol should be recognizable when seen for the first time by an unfamiliar driver. It is assumed that all details are essential, or at least helpful, in understanding the symbol's meaning. While the driver may have 
to be fairly close to the sign to recognize it initially, upon seeing the symbol on subsequent occasions, learning will occur whereby visibility of the small details will not be necessary, as the general (large detail) features of the symbol will be sufficient to convey its meaning at a distance.

\section{Common Sign Features}

$\underline{\text { Arrowheads }}$

The visibility of "ahead arrows" could be enhanced by using a more sharply angled arrowhead with rounded comers in combination with a narrower shaft to prevent contour interaction between the two when seen from a distance. An example of such an arrowhead appears in the modified KEEP RIGHT and DIVIDED HIGHWAY ENDS signs.

\section{$\underline{\text { Letter Symbols }}$}

Some symbol signs use a letter of the alphabet to convey (e.g., the P in NO PARKING, the H in HOSPITAL) or modify (e.g., the D in DIESEL FUEL) their messages. Since such letter symbols generally do not occupy the full space available for them, their legibility distance can be increased simply by making them larger. They should not, however, be extended so close to the border of the sign panel as to create contour interactions with it.

\section{Specific Symbol Elements}

It is most appropriate to use silhouette (side) views of vehicles in cases where the end view is likely to have poor legibility distance or comprehension, or where there may be confusion among messages (e.g., bus station, bus stop). Such a configuration typically contains distinctive features that enhance understanding (e.g., hood/trunk of a car; cab of a truck).

\section{Driver Age, Lighting, And Visual Functioning}

The legibility distance of symbol signs is reduced at night and under glare conditions. Legibility is also inversely related to driver age, a deficit that is most marked past middle-age (i.e., past about age 60). In table 56, where mean legibility distances are presented as a ratio of the daytime legibility of young drivers $(=1.0)$, it is clear that the age deficit is very much exacerbated by nighttime glare conditions. The legibility ranking of different signs, however, remains virtually unchanged across various lighting conditions and different age groups. This means that when the legibility of a sign is optimized in one lighting condition for a particular age group, it will be optimized for other lighting conditions and age groups as well.

Table 56. Theoretical values for predicting age differences in legibility distance.

\section{Viewing Condition}

\begin{tabular}{|c|c|c|c|}
\hline Age & $\underline{\text { Day }}$ & $\underline{\text { Night }}$ & $\underline{\text { Night/Glare }}$ \\
\hline Young & 1.00 & 0.70 & 0.69 \\
\hline Middle-aged & 0.88 & 0.60 & 0.60 \\
\hline Old & 0.80 & 0.46 & 0.34 \\
\hline
\end{tabular}


When legibility is defined as the ability to identify even the smallest of a sign's critical features, not surprisingly, the task is highly dependent on good visual acuity. Photopic (daytime) acuity was a strong predictor of legibility distance across age groups, particularly for non-glare conditions. The age-related loss of acuity undoubtedly contributes importantly to older drivers' legibility deficit. Loss of contrast sensitivity at spatial frequencies below those assessed by photopic acuity may be involved at nighttime or under glare conditions. Acuity and high cutoff spatial frequency were about equally well related to nighttime legibility. The best predictor of nighttime legibility under glare conditions was log peak sensitivity in the same lighting conditions. Given that the effect of a veiling glare is to reduce image contrast, it is not too surprising that the susceptibility of a symbol sign to glare effects can be best determined among observers who have difficulty seeing under such conditions.

It can be seen that the average legibility distance of symbol traffic signs for the older group of drivers under conditions of night-with-glare is about 1/3 of that for the young drivers under daytime viewing conditions. As compared with the daytime condition, the legibility distances decreased under night and night-with-glare conditions by about 30 percent for the young drivers. However, for the older drivers, this decrement was 43 percent and 57 percent, respectively, under these two nighttime conditions. The most disproportionate reduction occurred for the older drivers in the night-with-glare condition. These differences illustrate dramatically the need to take into account the limitations of the older driver, especially at night, when designing traffic sign symbols.

\section{Standards For Legibility Distance}

The argument for the establishment of standards for symbol comprehension applies as well to legibility distance. Although there are standards for sign placement (e.g., the STOP AHEAD sign on a roadway with 85 th percentile speed of $72 \mathrm{~km} / \mathrm{h}(45 \mathrm{mi} / \mathrm{h})$, should be placed

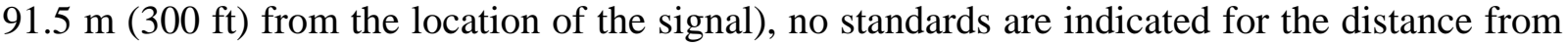
which symbols should be legible to drivers. The only relevant standard is the general guideline that legibility distances should be greater for higher speed roadways. Rigid legibility distance criteria may not be appropriate, since the placement of signs may be limited by roadway design and environmental factors (e.g., sharp curves, hills, rock outcroppings near the road).

The main factor determining legibility distance, and upon which standards should be based, is the time the driver needs to read, interpret, and act upon the information on the sign. This depends on operating speed, complexity of the maneuver, and roadway design (e.g., greater time is required to change lanes to exit a freeway from the left lane of a three-lane, as compared to a two-lane, freeway in response to guide sign information).

\section{Legibility Guidelines}

- Feature size. Other things being equal, larger features are legible from greater distances than are small ones. A sign's “critical” features should be as large as possible while remaining consistent with guidelines for ease of comprehension. To maximize contour size, solid rather than outline figures are preferable. 
- Feature separation/gap size. Contour interactions between elements of the same feature, or between adjacent features, can make a feature unrecognizable. Separation from other features and the border of the sign should be maximized.

- Figural simplicity. The contradictory demands for maximal feature size and separation can be reduced by making figures, as well as the elements which compose them, as simple as possible. The CROSS ROAD symbol, which has the greatest legibility distance of any sign in the MUTCD is perhaps the best example of an optimal combination of feature size, contour separation, and figural simplicity. Conversely, the poor legibility distance of the NO HITCHHIKING sign (the worst in the MUTCD), is attributable to its inadequacies in regard to these same three properties.

- Feature familiarity. Because the legibility and comprehension of familiar forms exceeds that for unfamiliar ones, the use of pictorially realistic features is preferable to more abstract or stylized forms.

- Contrast. Although restricted by the color coding that characterizes different sign types, maximizing the luminance and color contrast between adjacent features and also their background will enhance their legibility. Maximizing reflectivity differences between adjacent surfaces will similarly enhance legibility distance under nighttime conditions.

- Color. Although its coding importance mandates their use, the legibility of red contours (e.g., prohibitive circle and slash) is very low, especially at low light levels. The use of red contours should be restricted when possible, and otherwise, they should be made as large as possible, consistent with separation and size guidelines for other features on the sign.

\section{Enhancing Legibility Through Low-Pass Image Filtering}

Typically, the legibility of an unfamiliar sign is limited by the small size and/or contour interactions of one or more of its critical features. Because problems of size or contour interaction are exacerbated by low-pass filtering, the relative susceptibility of a sign's components to such effects can be used as a metric for identification and remediation. Kline and Fuchs, for example, showed that the legibility distance of selected symbol signs could be increased by approximately 50 percent when the features limiting their legibility were redesigned to enhance their resistance to strong optical blur. ${ }^{(57)}$ Although subjective, such a low-pass optical approach provides a highly cost-effective basis for improving symbol legibility. Unlike an appropriately configured image-processing system, however, the optical approach does not yield an objective metric for determining the spatial frequency components of displays, nor does it provide a straightforward means for relating differences in spatial vision to display legibility. For these reasons, it may be most useful in the initial design stages or when a computer imageprocessing system is unavailable to the designer.

The legibility of symbols can be enhanced using a low-pass image-processing approach such as that depicted in figure 16. Video images of symbol signs are digitized into the computer using a video processing board and custom software. They are then filtered through a progressive series of low-pass filters to identify features and feature interactions most vulnerable to loss of high spatial frequency information (i.e., to low-pass filtration). Once identified, these 
are shifted toward lower spatial frequencies (increased in size or separation) to increase their resistance to filtering. This process can be repeated to achieve the lowest cutoff threshold for feature or feature location that is consistent with other design constraints. The maximum cutoff threshold of a sign's critical feature(s) appears to be a powerful determinant of its legibility. Regardless of driver age, the correlation between low-pass cutoff threshold of signs and their daytime legibility is close to .90 , a level well within acceptable limits of test-retest reliability for the same measure.

\section{GLANCE LEGIBILITY}

Glance legibility is highly correlated $(r=.80)$ with both day and night legibility distance and appears to be based largely on the same design features. As for legibility distance, glance legibility varies greatly from one sign to another, and is worst for detailed or figurally complex symbols. Also, glance legibility is lower among older drivers, an age difference that is exacerbated by poor signs. Since glance legibility can be optimized by making symbols easily comprehended and by following the design principles described above for maximizing legibility distance, it need not be treated as a separate dimension in the design process.

\section{REACTION TIME}

Although there are marked differences across symbols in the latency with which drivers can match them to their text names, this does not appear to be related to their figural properties. Contextual variables such as familiarity and the similarity of alternative signs seem to be more important determinants of response latency. Consistent with a general age-related slowing in behavior, the response latencies of older drivers are considerably greater than those of their younger and middle-aged counterparts. Consequently, reaction time, at least as assessed by label-matching tasks, does not appear to be a very useful design dimension. Adherence to the following suggestions, however, would enhance likelihood that signs would be responded to in a timely and effective manner.

- Sign placement. Avoid placing signs which are similar in regard to color, features or message in close proximity.

- Sign familiarity/comprehension. Reaction time can be decreased by making symbols as easily understood as possible, and by using familiar symbols where appropriate.

- Advance warning. In critical situations, give drivers more response time by providing advance warning through early and if needed, multiple signs (e.g., SCHOOL BUS STOP AHEAD).

\section{CONSPICUITY}

The conspicuity of symbol signs varies markedly, however, this variation appears to be primarily a function of the relationship between a sign and the background in which it is seen than of properties of the sign itself. For example, conspicuity depends on nearby distracters, including other signs, level of illumination, and weather conditions. Further, the conspicuity of signs can vary considerably from one season to another in regions that experience significant changes in such environmental conditions as snowfall and level and color of foliage. Although 
the conspicuity search times of older drivers exceed those of younger ones, the difference appears to reflect the general age-related slowing in psychomotor functioning rather than something unique to symbol sign conspicuity. Older drivers appear to trade speed in favor of accuracy, so age differences in search accuracy are minimal. Because it is contextually determined, conspicuity has little utility as a design property in the creation or modification of individual symbol signs. The following guidelines can enhance a sign's conspicuity:

- Avoid sign clutter. The proximity of nearby similar signs can reduce the conspicuity of individual signs, especially if they are similar in size, features or color.

- Figural uniqueness. To enhance their conspicuity, particularly those that could be placed in close proximity to each other, symbols should be made as different as possible from one another.

- Sign placement. Where possible avoid placing signs against similar backgrounds (e.g., a green sign against green foliage).

- Distinct homogeneous background. Where distracting background or sign clutter are issues, the problem could be addressed by placing the sign on a large panel to provide a distinct background that makes the message more conspicuous.

\section{A SEQUENTIAL-COMPONENT APPROACH TO SIGN DESIGN AND MODIFICATION}

For symbol signs to be effective they must be easily and quickly understood and also legible from the greatest distance possible. Design features which contribute positively to one of these dimensions, however, may be of little help or even detract from one another. The challenge of developing signs could be reduced by employing a sequential-component approach in which features are added to the "base design" for the symbol in discrete stages. This base design would consist of the minimum elements needed to present the sign's message to naive viewers (i.e., the "critical elements"). From there, the elements that bring about the greatest increment to understanding would be added one at a time until a pre-determined threshold level of comprehension was reached. This would have the effect of keeping the design elements as few and as simple as possible. Once the minimal feature configuration for a sign was established in this way, the legibility of the design elements would be optimized, perhaps utilizing a low-pass filtering process described above. Only after the sign's critical elements were determined, and their legibility maximized, would additional features that enhanced a sign's comprehension (i.e., its educational features), and thus its legibility from global characteristics, be added (see also the section on Symbol Details). For example, if the sequential component approach were applied to the SCHOOL BUS STOP AHEAD sign, the base design might consist only of a large outline drawing to a school bus and an "ahead arrow." If this did not yield the acceptable level of comprehension, features that most increased understanding (e.g., rear doors, a student or students) could be added. The legibility of the sign could then be maximized using a low-pass filter process. If desired, and its layout permitted it, additional "educational" elements could be added (e.g., "flashing red lights," rear windows, books or a backpack) to increase the sign's legibility through the subsequent "top-down" benefits of enhanced comprehension.

\section{Methodology For Development Of Symbols}


The present series of studies was concerned with the evaluation of current symbols, recommendations for their improvement, and guidelines for symbol sign design and evaluation. For the novel symbols, and those symbols which were revised, only a single version was evaluated. In the development of symbols, where there are no existing candidates (e.g., from other signing systems), or where existing versions were known to be inadequate, the appropriate initial step would be to generate a number of candidate designs, by having drivers draw symbols to represent the message of interest. From this process, three or four versions differing in basic content and design could be compared on the various measures of sign effectiveness, as was done in the present project.

It should be recognized that the level of comprehension for an individual driver is in part a function of that driver's need for the information. For example, if one is looking for a train station, then locating and understanding that symbol are likely to be enhanced relative to a driver not seeking this information. Data from studies such as this may, therefore, underestimate the actual comprehension level that would be obtained in context by the driver who is searching for the information. One could measure this by having drivers, in a simulator study, "drive" on a scripted trip to specific destinations, where directional and other information was presented on symbol signs. These observations apply only to guide and recreational/cultural signs, as all drivers must know and respond to warning and regulatory symbols. 
APPENDIX A

FHWA SYMBOL HIGHWAY SIGNS

\section{Regulatory Signs}

Name

No Pedestrian

Crossing

No Right Turn

No U-Turn

Straight or Left

Keep Right

No Trucks

No Bicycles

No Parking

No Hitchhiking

National Network

Route

National Network

Prohibited

Mandatory Seat Belt

\section{$\underline{\operatorname{MUTCD}(\#)}$}

R9-3a

R3-1

R3-4

R3-6

R4-7

RS-2

RS-6

RS-3a

R9-4a

R14-4

R14-5

R16-1
Size

24 " x 24"

$24 " x 24 "$

$24 " x 24 "$

$30 " x 36$ "

$24 "$ x 30 "

$24 "$ x 24 "

$24 "$ x $24 "$

$24 "$ x 24"

$18 "$ x $18 "$

$24 "$ x $24 "$

$24 " x 24 "$

$15 " x 20 ”$

\section{Color}

Black \& Red on

White

Black \& Red on

White

Black \& Red on White

Black on White

Black on White

Black \& Red on

White

Black \& Red on

White

Black \& Red on

White

Black \& Red on White

Green and Black on White

Black \& Red on White

Black on White

\section{Warning Signs}

Turn (Right)

Curve (Right)

Reverse Turn (Right)

Reverse Curve (Right)

Winding Road (Right)

Large Arrow

Double Head Arrow

Chevron Alignment

Cross Road

Side Road

(Right, 90)

Side Road

(Right, 45")

\begin{tabular}{|c|c|c|}
\hline WI-I & $30 " x$ 30" & Black on Yellow \\
\hline W1-2R & $30 " x$ 30" & Black on Yellow \\
\hline W1-3R & $30 " x$ 30" & Black on Yellow \\
\hline W1-4R & $30 " x$ 30" & Black on Yellow \\
\hline W1-5R & $30 " x$ 30" & Black on Yellow \\
\hline Wl-6 & $48 " x 24 "$ & Black on Yellow \\
\hline Wl-7 & $48 " x$ 24" & Black on Yellow \\
\hline W1-8 & $18 " x 24 "$ & Black on Yellow \\
\hline W2-1 & $30 " x$ 30" & Black on Yellow \\
\hline W2-2 & $30 " x$ 30" & Black on Yellow \\
\hline W2-3 & $30 " x$ 30" & Black on Yellow \\
\hline
\end{tabular}

Note:

MUTCD \# = Manual of Uniform Traffic Control Device Reference Number 
Warning Signs (continued)

\begin{tabular}{|c|c|c|c|}
\hline T Symbol & W2-4 & $30 ”$ x 30” & Black on Yellow \\
\hline Y Symbol & W2-5 & $30 ” \times 30 ”$ & Black on Yellow \\
\hline Stop Ahead & W3-la & $36 " x 36 "$ & $\begin{array}{l}\text { Black and Red } \\
\text { on Yellow }\end{array}$ \\
\hline Yield Ahead & W3-2a & $36 " x 36 "$ & $\begin{array}{l}\text { Black, Red, \& } \\
\text { White on Yellow }\end{array}$ \\
\hline Signal Ahead & W3-3 & $36 " x$ x 36" & $\begin{array}{l}\text { Green, Black, \& } \\
\text { Red on Yellow }\end{array}$ \\
\hline Merge & W4-1 & $30 " x 30 "$ & Black on Yellow \\
\hline $\begin{array}{l}\text { Added Lane } \\
\text { Lane Reduction } \\
\text { Transition }\end{array}$ & $\begin{array}{l}\text { W4-3 } \\
\text { W4-2 }\end{array}$ & $\begin{array}{l}36 " x \text { x } 36 " \\
36 " x \text { x } 36 "\end{array}$ & $\begin{array}{l}\text { Black on Yellow } \\
\text { Black on Yellow }\end{array}$ \\
\hline Narrow Bridge & W5-2a & $30 "$ x 30" & Black on Yellow \\
\hline Divided Highway & W6-1 & $36 " x$ 36" & Black on Yellow \\
\hline Divided Highway Ends & W6-2 & $36 " x$ 36" & Black on Yellow \\
\hline Two-Way Traffic & W6-3 & $30 "$ x 30" & Black on Yellow \\
\hline Hill & W7-1 & $30 ”$ x 30” & Black on Yellow \\
\hline Pavement Ends & W8-3a & $36 " x$ 36" & Black on Yellow \\
\hline Slippery When Wet & W8-5 & $30 " x$ 30" & Black on Yellow \\
\hline Bicycle Crossing & W11-1 & $30 "$ x 30" & Black on Yellow \\
\hline Pedestrian Crossing & Wlla-2 & $30 " x$ x $30 "$ & Black on Yellow \\
\hline Deer Crossing & W11-3 & $30 " x$ 30" & Black on Yellow \\
\hline Cattle Crossing & W11-4 & $30 " x$ 30" & Black on Yellow \\
\hline Tractor Crossing & W11-5 & $30 " x$ 30" & Black on Yellow \\
\hline Double Arrow & W12-1 & $24 " \times 24 "$ & Black on Yellow \\
\hline Low Clearance & W12-2 & $36 " x$ 36" & Black on Yellow \\
\hline Playground & W15-1 & $36 " \times 36 "$ & Black on Yellow \\
\hline Advance Flagger & W20-7a & $36 " x$ 36" & Black on Orange \\
\hline Worker (Construction) & W21 -la & $36 " x 36 "$ & Black on Orange \\
\hline
\end{tabular}

\section{Guide Signs}

Rest Area
Phone
Hospital
Camping
Recreational Vehicle
Handicapped
Gas
Food
Lodging
Tourist Information
Diesel
Recreational Vehicle
Sanitary Station

\begin{tabular}{|c|c|}
\hline D5-5a & $24 "$ x 24" \\
\hline D9-1 & $24 "$ x 24" \\
\hline D9-2 & $24 " x 24 "$ \\
\hline D9-3 & $24 " x$ 24" \\
\hline D9-3a & $24 " \times 24 "$ \\
\hline D9-6 & $24 "$ x 24" \\
\hline D9-7 & $24 "$ x 24" \\
\hline D9-8 & $24 "$ x 24" \\
\hline D9-9 & $24 " x 24 "$ \\
\hline D9-10 & $24 "$ x 24" \\
\hline D9-11 & $24 "$ x 24" \\
\hline D9-12 & $24 " x 24 "$ \\
\hline
\end{tabular}

White on Blue White on Blue White on Blue White on Blue White on Blue White on Blue White on Blue White on Blue White on Blue White on Blue White on Blue White on Blue 


\section{Guide Signs (continued)}

Emergency Medical

D9-13

Services

Propane

Hiking Trail

Airport

Bus Station

Train Station

Library

Dock

Winter Recreation
D9-15

I-4

I-S

I-6

I-7

I-82

I-9

I-10
$24 " x 24 "$

$24 " x 24$ "

24 " x 24 "

$24 " x 24 "$

$24 "$ x $24 "$

$24 "$ x $24 "$

$24 "$ x 24 "

$24 "$ x 24 "

$24 "$ x 24 "
White on Blue

White on Blue

White on Green

White on Green

White on Green

White on Green

White on Green

White on Green

White on Green

\section{Recreational and Cultural Interest Area Signs}

Lighthouse

Ranger Station

RG-120

RG-170

Rest Rooms

Campfire

RM-140

RA-030

Shelter (Sleeping)

Showers

Amphitheater

Canoeing

Ramp (Launch)

Swimming

Skating (Ice)

Sledding

Snowmobiling
RA-110

RA-130

RL-010

RW-020

RW-080

RW-130

RS-010

RS-060

RS-070
24 " x 24"

$24 "$ x 24"

24 " x 24"

$24 "$ x 24"

24 " x 24"

$24 "$ x 24 "

24 " x 24 "

24 " x 24 "

24 " x 24 "

24 " x 24 "

24 " x 24"

24 " x 24"

24 " x 24 "
White on Brown White on Brown White on Brown White on Brown

White on Brown White on Brown White on Brown White on Brown White on Brown White on Brown White on Brown White on Brown White on Brown

\section{School Area Signs}

School Advance

School Crossing

S2-1

$36 " x 36 "$

$36 " \times 36 "$

Black on Yellow

Black on Yellow

\section{Railroad - Highway Grade Crossing Signs}

Railroad Advance

Warning

Railroad Advance

Warning (Railroad

Parallel to Roadway)
W10-1

36" Diameter Black on Yellow

W10-3

$30 " x 30 "$

Black on Yellow 


\section{APPENDIX B}

\section{SAMPLE CONSENT FORM}

\section{$\underline{\text { Summary }}$}

We are interested in finding out how well drivers understand the meanings of symbol traffic signs. You will be shown slides of a number of traffic signs used on highways and will be asked to write the meaning of each sign on a booklet. If you are unsure of what any specific sign means, then take a guess. If you have no idea what it means, then write "don't know" in the answer blank. The specific procedure will be described to you before we begin.

You will also be asked to provide some general background about yourself, such as age and driving experience.

The information you provide and your results will be kept strictly confidential---only the experiementer will have access to this information. Results will be reported as group averages and your name will not be associated with the findings.

\section{$\underline{\text { Informed Consent }}$}

I have read the above summary and I understand that my participation in this study is completely voluntary and that I may, without any penalty, withdraw from participation at any time or refuse to answer any questions that I am asked. I also understand that any information gathered from me in the course of this study will be strictly confidential, and except as part of an overall group average, will be released to no one but me upon my written request. I also recognize that the data will be stored in a locked location until it is destroyed.

Signed:

(Participant)

Date:

(Experimenter) 
Subject Code:

\section{Name:}

\section{Sex: Male/Female}

Age:

Occupation:

1. How many years have you been driving in total? years

2. How many years have you held a driver's license in total? years

3. The number of years of formal schooling that you have completed is:

1 to 8 years ........................................................................ 1

Some high school ............................................................. 2

High school graduate ...................................................... 3

Trade school or business college ................................ 4

Some college or university .......................................... 5

University degree.......................................................... 6

Post graduate education or degree.............................. 7

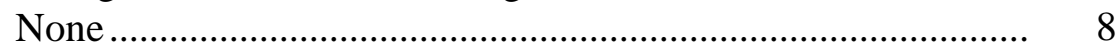

4. What type of motor vehicle do you drive most of the time?

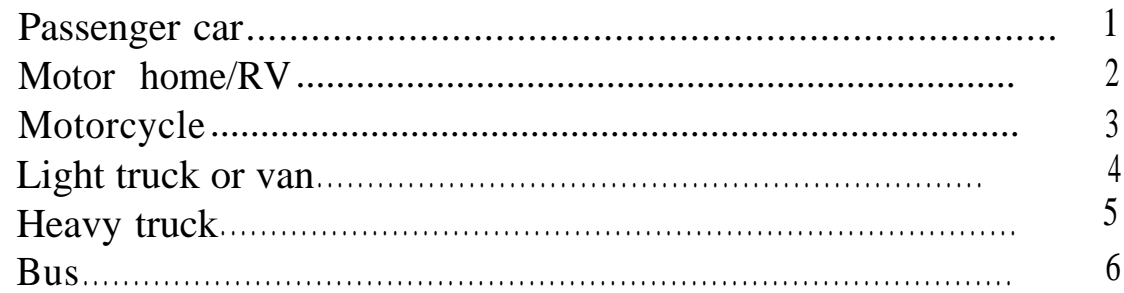

5. How many miles (or kilometres) a year do you drive?

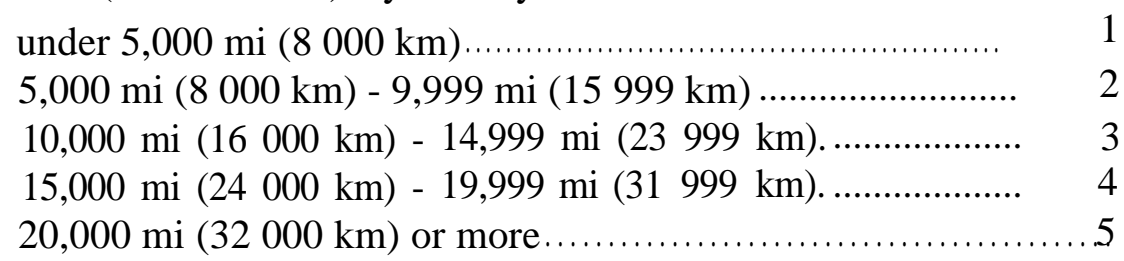


6. Over the last 12 months, what percentage of your driving would you estimate was done at night?

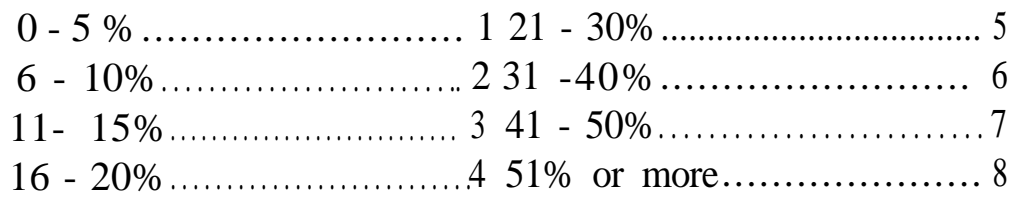

7. Over the last 12 months, what percentage of your driving would you estimate was done during rush hour?

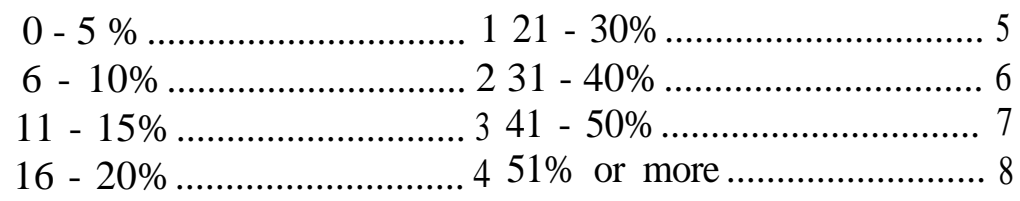

8. Over the last 12 months, what percentage of your driving would you estimate was done on the highway (rather than around town)?

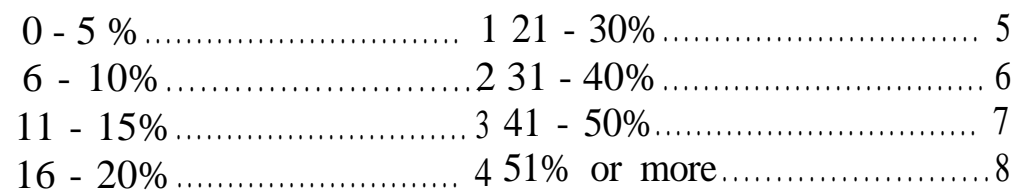

9. In what kind of environment do you do most of your driving?

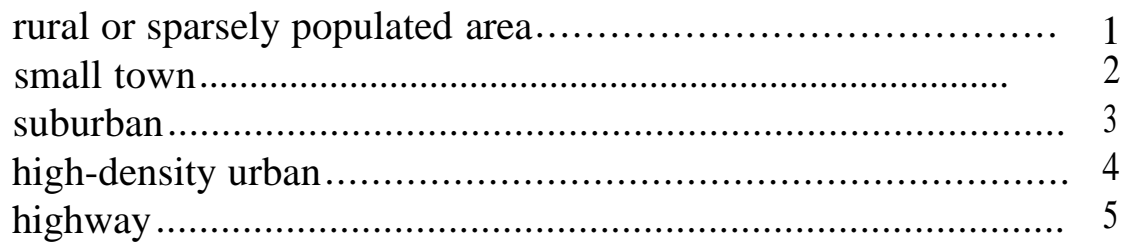

10a. Do you have any professional driving or highway-safety experience (e.g., as a cab, bus, or truck driver, a highway engineer, etc.)?

$$
\text { No }
$$

10b. If your answer is "Yes," please indicate below the kind of experience that you have had, when it occurred, and for how many years it lasted. 


\section{APPENDIX D}

\section{LEGIBILITY DISTANCE SCORING KEY}

$\underline{\text { Abbreviations }}$
1. w/
$=$ with
2. (parenthesis)
$=$ correct alternative or additional information
3.;
= separates different correct answers

Slide No.

Practice

1.

2.

3

4

5

6

7

8.

9. $\underline{\text { Slide Name }}$

No Pedestrians

No Right Turn

No U-Turn

Straight or Left

Keep Right

No Trucks

No Bicycles

No Parking

No Hitchhiking

National Network

Route $\underline{\text { Key Features }}$

Red circle around \& diagonal slash over person facing left

Red circle around \& diagonal slash over arrow that bends $90^{\circ}$ right

Red circle around \& diagonal slash over inverted $U$ arrow, w/ arrow (head) on left

Two joined arrows: one pointing straight up and one pointing left

Island (barrier) on left, arrow veering around it to right

Red circle around \& diagonal slash over truck (silhouette) (facing left)

Red circle around \& diagonal slash over bicycle (outline, silhouette) (facing left)

Red circle around \& diagonal slash over letter $\mathrm{P}$

Red circle around \& diagonal slash over fist $\mathrm{w} /$ extended thumb

Green circle around rear view of truck, two sets of wheels, or rectangle w/ concave or chunk out of bottom 
10.

National Network

Route Prohibited

11.

12.

14.

15.

16

17.

18.

19.

20.

21

22

23.

24.

25.
Mandatory Seat Belt

Right Turn

Right Curve

Right Reverse Turn

Right Reverse Curve

Winding Road (Right)

Large Arrow

Double Head Arrow

Chevron Alignment

Cross Road

Side Road

(90 degrees)

Side Road

(45 degrees)

T Symbol

Y Symbol

Stop Ahead
Red circle around and diagonal slash over rear view of truck, w/ two sets of wheels, or rectangle w/ concave or chunk out of bottom

Seated figure outline, w/ seat belt from shoulder across hips or waist

Up arrow turns 90 degrees to right

Arrow curving up to upper right

Arrow turns 90 degrees right and then to point straight up

Up arrow w/ (gentle) curve right, and ending w/ arrowhead pointing up

Arrow pointing up in S-curve

Arrow (head) pointing right

One arrow w/ two arrowheads, one to right and one to left

Arrowhead (chevron) facing right

$90^{\circ}$ intersecting horizontal and vertical lines; plus sign

Vertical line joined at centre by (half) (right, $90^{\circ}$ ) horizontal line from right

Vertical line w/ second line branching (right 45") (from centre) up to right

$\mathrm{T}$ intersection; (capital) $\mathrm{T}$

Y intersection; (capital) $\mathrm{Y}$

Arrow (head) point up w/ red octagon (stop sign) below it 
Added Lane

30.

31.

33.

34.

35.

36.
Divided Highway

Begins

Divided Highway

Ends

Lane Reduction

Transition

Narrow Bridge

Two-Way Traffic

Hill

Pavement Ends

Slippery When Wet
Arrow (head) pointing up, red /white triangle point down (yield sign) below it

At least 2 of 3 coloured circles: red, green, yellow; traffic signals

Arrow pointing up w/ line joining from lower right at an angle

Two arrows: one straight up, one curving from lower right, (triangular) divider between arrows

Straight vertical line on right bowed in (curved) vertical line on left

$\mathrm{H}$-shape squeezed in middle, dashed line down centre of figure

Two arrows, left arrow pointing down, right arrow pointing up, island (triangle, barrier) at top between arrows

Two arrows, left arrow pointing down, right arrow pointing up, island (triangle, barrier) at bottom between arrows

Two arrows, left pointing down, right pointing up

Truck on hill (grade, ramp, wedge, slope) sloping down toward left

Gravel road (mottled or speckled rectangle) at top w/ paved road (solid rectangle) at bottom w/ roadway markings (dashed lines)

Car w/ skid (wavy) lines below it 
26.

Yield Ahead

27.

Signal Ahead

28

Merge

29.

Added Lane

30.

31

32.

33.

34

35
Divided Highway

Begins

Lane Reduction

Transition

Narrow Bridge

Divided Highway

Ends

Two-Way Traffic

Hill

Pavement Ends

Slippery When Wet
Arrow (head) pointing up, red /white triangle point down (yield sign) below it

At least 2 of 3 coloured circles: red, green, yellow; traffic signals

Arrow pointing up w/ line joining from lower right at an angle

Two arrows: one straight up, one curving from lower right, (triangular) divider between arrows

Straight vertical line on right, bowed in (curved) vertical line on left

$\mathrm{H}$-shape squeezed in middle, dashed line down centre of figure

Two arrows, left arrow pointing down, right arrow pointing up, island (triangle, barrier) at top between arrows

Two arrows, left arrow pointing down, right arrow pointing up, island (triangle, barrier) at bottom between arrows

Two arrows, left pointing down, right pointing up

Truck on hill (grade, ramp, wedge, slope) sloping down toward left

Gravel road (mottled or speckled rectangle) at top $\mathrm{w} /$ paved road (solid rectangle) at bottom $\mathrm{w} /$ roadway markings (dashed lines)

Car w/ skid (wavy) lines below it 
38.

Bicycle Crossing

39.

40.

41.

42.

44.

45.

46.

47.

48.

49.

50.

51.

52.

53.
Pedestrian Crossing

Deer Crossing

Cattle Crossing

Tractor Crossing

Double Arrow

Low Vertical

Clearance

Playground

Advance Flagger

Worker

(Construction)

Rest Area

Phone

Hospital

Camping

Recreational Vehicle

Handicapped
Bicycle (outline, silhouette), heading left

Figure walking left, horizontal line or lines across bottom

Deer, (antelope, elk, caribou) jumping left

Cow (bull) facing left

Figure (person, farmer, man)

riding tractor, facing left

Two arrows pointing down, one to right and one to left

Two arrowheads, one pointing up, other down, w/ numbers between arrows

Figures (human, kids, children) on each end of teeter-totter (see-saw)

Figure (human, person, man) on right, holdering flag straight out on left

Figure (human, person, man) lump (dirt pile) on right

(Cross-section) picnic table and a spruce (fir, pine, Christmas tree) on right

Telephone (receiver)

$\mathrm{H}$

Tent w/ smaller triangle or door

Trailer w/ two windows

Wheel and seated figure (person); wheelchair w/ person 
54.

Gas

55.

Food

56.

57.

58.

59

60.

61.

62

64.

65.

66.

67.

68.

69.

70.
Lodging

Tourist Information

Diesel

RV Sanitary Station

Emergency Medical

Services

Propane

Hiking Trail

Airport

Bus Station

Train Station

Library

Dock

Winter Recreation

Lighthouse

Ranger Station
Fuel (gas) pump

Knife on right, fork on left

Bed w/ figure lying on it

Question mark

Fuel (gas) pump w/ letter D

Trailer above hole, w/ arrow

Three-way cross or asterisk w/ staff (stick)

Propane cylinder (bottle); two curved sides, larger rectangular top and smaller rectangular bottom

Two figures (people, man and woman) walking left w/ backpacks

Airplane

Bus w/ windows; front end of bus

Train, building, person (figure, passenger)

Top half of human figure (person) w/ book in hand

Anchor; semi-circle plus vertical stem

Snowflake; white crisscrossing diagonals and verticals w/ holes

Lighthouse; vertical rectangle w/ door, narrowing toward top

Standing human figure (ranger, warden) in hat on left, building \& flag right 
71.

Rest Rooms

72.

Campfire

73

74.

75

76

77.

78.

79

80.

81.

82

84.

85
Shelter (Sleeping)

Showers

Amphitheater

Canoeing

Launch Ramp

Swimming

Ice Skating

Sledding

Snowmobiling

School Advance

School Crossing

R.R. Advance

Warning

R.R. Advance

Warning (Railroad

Parallel to Roadway)
Woman (female figure) on left, man (male figure) right, each enclosed in a sloping rectangle

Campfire; flames on logs; burning $\log s$

Shelter or (roof, wall, \& floor) w/ prone figure

Showerhead \& figure w/ droplets; or lamp w/ dots /light rays

Amphitheatre; three curved lines (semicircles, arcs) below square

Canoe (horizontal curved thing) w/ two people (figures, canoeists)

Boat on a ramp, sloping down right

Person (figure, swimmer)

swimming to right, waves/water

Skater, (figure, person) w/ skates (not skis); person skating

Person (figure, child) on tummy, toboggan, (sled, sleigh)

Person (figure), riding ski-doo or snowmobile

Two persons (figures, kids)

walking left

Two persons (figures, kids, children) walking left, two parallel lines

Diagonal cross w/ letter R on right and left

Vertical road w/ right angle road crossing vertical train tracks on right 


\section{APPENDIX E}

\section{COMPREHENSION SCORING KEY}

$\mathrm{C}=$ correct $; \mathrm{P}=$ partially correct $; \mathrm{W}=$ possible wrong responses

1. No Right Turn

C no right turn; do not turn right; go straight or left

W turn right; no turns; keep left; no turn on red

2. No U-Turn

C no U turn; go straight, left or right

W no turns

3. Straight Or Left (Lane Control)

C this lane goes straight or left; don't turn right from this lane; must go straight or left

in this lane

W left turn lane; no right turn

4. Keep Right

C keep right; hazard keep right; no going left

$\mathrm{P} \quad$ hazard on the left; dirt pile, keep right; hazard ahead, keep right

5. No Trucks

C no trucks; trucks prohibited

P cars, motorcycles and bikes only

W no vehicles allowed

6. No Bicycles

C no bicycles (allowed)

$\mathrm{P} \quad$ non-motorized vehicles prohibited

W no bike path

7. No Parking

C no parking; don't park here

W no stopping; no stopping or parking; restricted parking

8. No Hitchhiking

C no hitchhiking

W no pedestrians, no entry

9. National Network Route (Truck Route)

$\mathrm{C}$ (national) truck route; large trucks allowed;

$\mathrm{P} \quad$ trucks allowed

W truck parking; danger trucks; truck stop; no trucks 
10. National Network Prohibited (Trucks Prohibited)

C large trucks prohibited on this route/roadway; not a national truck route

$\mathrm{P} \quad$ no trucks (allowed)

W no truck parking; not a truck stop

11. Mandatory Seat Belts

C seat belts required (by law); use seat belts

P seat belts; seat belt laws

12. Turn (Right)

C right turn (in road); road turns to the right (ahead); sharp curve to the right

$\mathrm{P} \quad$ road curves to the right; right curve;

W turns ahead: curves ahead

13. Curve (Right)

C right curve (in road); road curves to right

$\mathrm{P} \quad$ right turn; sharp curve

W curves ahead; winding road

14. Reverse Turn (Right)

C reverse (S) turn or sharp curves, first turn to the right; 2 turns in opposite directions, first turn to the right

P reverse (S) turn; sharp reverse curve

W winding road (ahead); road goes right or left

15. Reverse Curve (Right)

C reverse (or S) curve first curve is to right;

2 curves in opposite directions, first curve is to right;

$\mathrm{P} \quad$ reverse $(\mathrm{S})$ curve; curves ahead

W winding road (ahead)

16. Winding Road

C (3 or more) curves/turns ahead; winding road; curves ahead

$\mathrm{P} \quad$ curve (ahead)

W slippery road

17. Large Arrow

C sharp curve to right; road alignment goes sharply right

$\mathrm{P} \quad$ right turn/curve

W one way road

18. Double Headed Arrow

C sharp turn to right and left; $T$ intersection

$\mathrm{P} \quad$ go left or right; road goes both ways

W road ends; stop ahead; yield ahead; two way traffic 


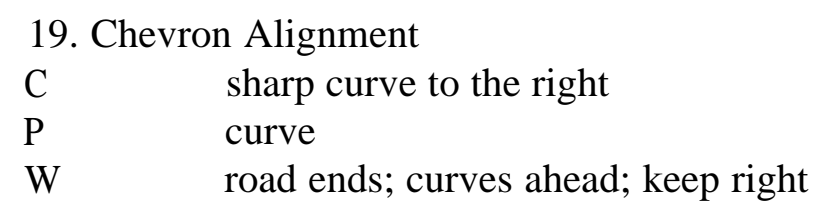

20. Cross Road

C cross road (ahead); intersection ahead; roads entering from left and right (ahead)

W stop ahead

21. Side Road (Right, $90^{\circ}$.)

C road enters from right; road intersected by $90^{\circ}$ road

$\mathrm{P} \quad$ intersection ahead; $\mathrm{T}$ intersection ahead

W merging traffic

22. Side Road (Right, $45^{\circ}$ )

C road enters/leaves from right at an angle

$\mathrm{P} \quad$ road entering/leaving from the right; intersection ahead

W merging traffic; exit ramp (ahead)

23. T Symbol

C T intersection (ahead); road goes (must turn) right or left

$\mathrm{P} \quad$ intersection ahead

W road ends; stop ahead; dead end; yield ahead

24. Y Symbol

C $\quad$ Y intersection (ahead); road goes right and/or left; road splits

$\mathrm{P} \quad$ intersection ahead

W road ends; exit ramp

25. Stop Ahead

C stop (sign) ahead

$\mathrm{P} \quad$ be prepared to stop

W stop; intersection ahead

26. Yield Ahead

$\begin{array}{ll}\text { C } & \text { yield (sign) ahead } \\ \text { P } & \text { be prepared to yield } \\ \text { W } & \text { yield }\end{array}$

27. Signal Ahead

C (traffic) signal or lights ahead; be prepared to stop

W intersection ahead

28. Merge

$\mathrm{C} \quad$ merge; merging traffic (from right)

$\mathrm{P} \quad$ traffic entering from the right; intersecting road; two lanes merge into one lane 
29. Added Lane

$\mathrm{C} \quad$ added lane; new lane ahead

P 2 (or more) lanes ahead

W merge; merging traffic; yield; lane ends

30. Lane Reduction Transition

C (right) lane ends; lane drop

$\mathrm{P} \quad$ merge left

W road narrows; two way traffic

31. Narrow Bridge

$\mathrm{C}$ narrow bridge

P road narrows; narrow lane; one lane bridge

W no shoulders ahead; bridge ahead

32. Divided Highway

C divided highway/road begins (ahead); roadway divides

P hazard or median ahead

W keep right; watch for opposing traffic

33. Divided Highway Ends

C end of divided highway; two way traffic ahead

$\mathrm{P} \quad$ freeway ends

W road narrows

34. Two-Way Traffic

C two-way traffic (ahead); opposing traffic

$\mathrm{P} \quad$ undivided highway

W 2 lanes ahead

35. Hill

C (steep) hill; downgrade

P steep hill for trucks

W truck stop; watch for trucks

36. Pavement Ends

C pavement/paved road ends; gravel (dirt) road begins

W rough road (ahead); centre line ends

37. Slippery When Wet

C slippery when (road) wet

W slippery road; icy road; curves; rough road

38. Bicycle Crossing

C bicycle (bike) crossing; watch for bicycles; bike path crosses road

W bicycle path; bicycles 
39. Pedestrian Crossing

C pedestrian crossing; crosswalk; stop for (yield to) pedestrians

$\mathrm{P} \quad$ watch for pedestrians

W school zone; children; playground; pedestrian pathway

40. Deer Crossing

C deer (animals) crossing; (wild) animals on/near road; wildlife

W zoo

41. Cattle Crossing

C cattle (stock) crossing; cattle/farm animals on (near) road

W petting zoo; dairy; farm

42. Tractor Crossing

C tractor (farm equipment) crossing or on the road

$\mathrm{P} \quad$ Tractor/farm equipment

W no tractors allowed; fanning area

43. Double Arrow

C keep right or left (of traffic island/obstruction); traffic may go either right or left

$\mathrm{P} \quad$ traffic island

44. Low Vertical Clearance

C low clearance; low bridge/overpass; (vehicle) height restriction (saying the number not essential here)

$\mathrm{P} \quad$ caution for high vehicles

W vehicle length restriction

45. Playground

C playground (zone);

$\mathrm{P} \quad$ children in area

W school zone

46. Advance Flagger

C flagger/flagman (ahead); obey flagger

$\mathrm{P} \quad$ be prepared to stop for construction

W construction

47. Worker (Construction)

C construction (zone); workers; road work

$\mathrm{P} \quad \mathrm{men} /$ workers and equipment on road

W detour; pet walking area

48. Rest Area

C rest area; roadside tables; picnic tables/area

$\mathrm{P} \quad$ turnout

W camping 
49. Phone

C phone (booth)

P emergency phone: call box

W phone and washroom

50. Hospital

C hospital

P medical services; first aid

51. Camping

C camping; campground

$\mathrm{P} \quad$ tenting

52. Recreational Vehicle

C camping for recreational vehicles; $\mathrm{R} \mathrm{V}$ park

$\mathrm{P} \quad$ camping

W lane for R V's; trailer disposal facilities

53. Handicapped

C handicapped facilities (available); handicapped

W wheelchair; hospital; handicapped crosswalk

54. Gas

C gas; fuel; gas station

$\mathrm{P} \quad$ service station

W auto repairs

55. Food

C food; restaurant; eating place; dining; coffee shop

$\mathrm{P} \quad$ snack bar

W groceries

56. Lodging

C lodging, accommodation; motel/hotel

W bed; furniture store

57. Tourist Information

C (tourist) information; information booth

W (emergency) road services

58. Diesel

C diesel; diesel fuel

$\mathrm{P} \quad$ fuel; gas; gas station

W auto repairs

59. RV Sanitary Station

C RV sanitary station; RV waste deposit

W RV camping; camping; RV parking 
60. Emergency Medical Services

C emergency medical (services); medical services; first aid

P hospital

61. Propane

C propane (gas/fuel)

W gas; fuel; service station

62. Hiking Trail

C hiking (trail); trail; trail marker

W picnic site; watch for hikers; hikers crossing

63. Airport

C (route to) airport

W airplane; runway; low flying aircraft

64. Bus

C (route to) bus terminal

W bus stop; bus lane; watch for buses

65. Train Station

C (route to) train/railroad station

W railroad crossing; watch for trains; station

66. Library

C (route to) library

W books; book store; reading room; information

67. Dock

C (boat) dock; marina; place for boats

$\mathrm{P} \quad$ wharf; jetty; boat mooring

W boat anchoring area; swimming; fishing

68. Snow

C winter recreation/sports

W snow; ski hill; icy road

69. Lighthouse

C lighthouse

W historical site

70. Ranger Station

C ranger (station)

W police; camping; guard house; military camp 


\section{Rest Rooms}

C rest rooms; wash rooms; toilet(s); men's and women's

W accommodation

72. Campfire

C campfire (allowed)

$\mathrm{P} \quad$ barbecue pit

W camping; fire (hazard); campfire program

73. Shelter (Sleeping)

C sleeping shelter; shelter for sleeping

$\mathrm{P} \quad$ outdoor accommodation; shelter

W accommodation; motel/hotel; rest area

74. Showers

C shower(s)

W washroom; toilet; street light

75. Amphitheater

C amphitheater; outdoor/open air theater

$\mathrm{P} \quad$ theater; arena;

W loud speaker; radio

76. Canoeing

C canoeing

P boating; kayaking

W canoe; canoe rental; boat rental

77. Launch Ramp

C boat ramp/launch; boat launching

$\mathrm{P} \quad$ ramp

W boating

78. Swimming

C swimming (allowed)

P beach; pool

W diving

79. Ice Skating

C (ice) skating

$\mathrm{P} \quad$ skating arena

W (ice) hockey; skiing

80. Sledding

C sledding; tobogganing; sliding

W bobsledding; bobsled/luge run; snowmobiling 
81. Snowmobiling

C snowmobiling; skidooing

W snowmobile crossing; sledding

82. School Advance Warning

C school (zone/area) ahead

$\mathrm{P} \quad$ school zone/area

W playground (zone/area); watch for children; school bus stop

83. School Crossing

C school crossing

P school zone/area; children crossing; school crossing (ahead)

W school (zone) ahead; watch for children

84. Railroad Advance Warning

C railroad/train crossing (ahead); trains cross the road (ahead); train tracks ahead

$\mathrm{P} \quad$ trains

W railroad intersection

85. Railroad Advance Warning (R R Parallel To Roadway)

$\mathrm{C}$ railroad crossing parallel to (beside) road; railroad crossing on side road

W cross road; intersection; side road 


\section{APPENDIX F}

18 FHWA SYMBOL SIGNS FOR NIGHTTIME LEGIBILITY, GLANCE LEGIBILITY/REACTION TIME, AND CONSPICUITY STUDIES

\begin{tabular}{|c|c|c|c|c|c|}
\hline $\begin{array}{l}\text { Sign } \\
\text { Category }\end{array}$ & $\begin{array}{l}\text { Sign } \\
\text { Name }(\mathrm{cm})\end{array}$ & $\begin{array}{l}\text { Sign } \\
\text { Ht \& W } \\
\text { inches } \\
(\mathrm{cm})\end{array}$ & & $\begin{array}{l}\text { Screen } \\
\text { Height }\end{array}$ & $\underline{\text { Color }}$ \\
\hline Warn(B) & Cross Road & $\begin{array}{l}42.43 \\
(107.77)\end{array}$ & 42.43 & 3.37 & Yellow, Black \\
\hline Warn(B) & Right Curve & $\begin{array}{l}42.43 \\
(107.77)\end{array}$ & 42.43 & 3.37 & Yellow, Black \\
\hline Warn(I) & Div Hwy Ends & $\begin{array}{l}50.91 \\
(129.31)\end{array}$ & 50.91 & 4.04 & Yellow, Black \\
\hline Warn(I) & Advance Flagger & $\begin{array}{l}50.91 \\
(129.31)\end{array}$ & 50.91 & 4.04 & Orange, Black \\
\hline Warn $(\mathrm{W})$ & Bicycle Crossing & $\begin{array}{l}42.43 \\
(107.77)\end{array}$ & 42.43 & 3.37 & Yellow, Black \\
\hline Warn(W) & Pavement Ends & $\begin{array}{l}50.91 \\
(129.31)\end{array}$ & 50.91 & 4.04 & Yellow, Black \\
\hline $\operatorname{Reg}(\mathrm{B})$ & No U-Turn & $\begin{array}{l}24 \\
(60.96)\end{array}$ & 24 & 1.91 & $\begin{array}{l}\text { Red, Black, } \\
\text { White }\end{array}$ \\
\hline $\operatorname{Reg}(B)$ & Keep Right & $\begin{array}{l}30 \\
(76.20)\end{array}$ & 24 & 2.38 & Black, White \\
\hline
\end{tabular}

Notes:

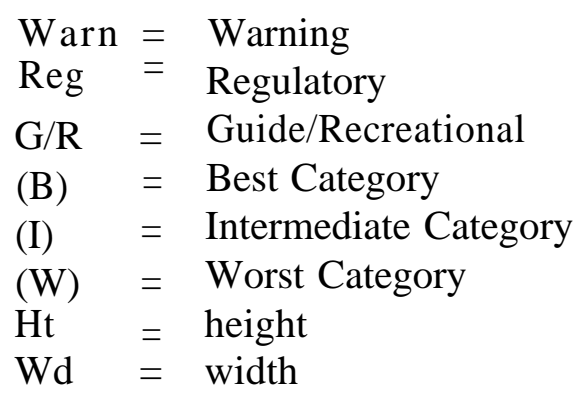




\begin{tabular}{|c|c|c|c|c|}
\hline $\begin{array}{l}\text { Sign } \\
\text { Category } \\
\end{array}$ & $\begin{array}{l}\text { Sign } \\
\text { Name } \underline{(\mathrm{cm})}\end{array}$ & $\begin{array}{l}\text { Sign } \\
\text { Ht \& Wd } \\
\text { inches } \\
(\mathrm{cm})\end{array}$ & $\frac{\text { Screen }}{\text { Height }}$ & $\underline{\text { Color }}$ \\
\hline $\operatorname{Reg}(I)$ & No Parking & $\begin{array}{l}24 \\
(60.96)\end{array}$ & 1.91 & $\begin{array}{l}\text { Red, Black, } \\
\text { White }\end{array}$ \\
\hline $\operatorname{Reg}(\mathrm{I})$ & No Trucks & $\begin{array}{l}24 \\
(60.96)\end{array}$ & 1.91 & $\begin{array}{l}\text { Red, Black, } \\
\text { White }\end{array}$ \\
\hline $\operatorname{Reg}(\mathrm{W})$ & $\begin{array}{l}\text { National Network } \\
\text { Route Prohibited }\end{array}$ & $\begin{array}{l}24 \\
(60.96)\end{array}$ & 1.91 & $\begin{array}{l}\text { Red, Black, } \\
\text { White }\end{array}$ \\
\hline $\operatorname{Reg}(\mathrm{W})$ & Mandatory Seat Belt & $\begin{array}{l}20 \\
(50.80)\end{array}$ & 1.59 & Black, White \\
\hline $\mathrm{G} / \mathrm{R}(\mathrm{B})$ & Hospital & $\begin{array}{l}24 \\
(60.96)\end{array}$ & 1.91 & Blue, White \\
\hline $\mathrm{G} / \mathrm{R}(\mathrm{B})$ & Phone & $\begin{array}{l}24 \\
(60.96)\end{array}$ & 1.91 & Blue, White \\
\hline $\mathrm{G} / \mathrm{R}(\mathrm{I})$ & RV Sanitary Stn & $\begin{array}{ll}24 & 24 \\
(60.96) & \end{array}$ & 1.91 & Blue, White \\
\hline $\mathrm{G} / \mathrm{R}(\mathrm{I})$ & Campfire & $\begin{array}{ll}24 & 24 \\
(60.96) & \end{array}$ & 1.91 & Brown, White \\
\hline $\mathrm{G} / \mathrm{R}(\mathrm{W})$ & Skating & $\begin{array}{ll}24 & 24 \\
(60.96) & \end{array}$ & 1.91 & Brown, White \\
\hline $\mathrm{G} / \mathrm{R}(\mathrm{W})$ & Ranger Station & $\begin{array}{ll}24 & 24 \\
(60.96) & \end{array}$ & 1.91 & Brown, White \\
\hline
\end{tabular}




\section{APPENDIX G}

\section{RESPONSE LATENCY: TEST SIGNS AND FOILS}

\section{$\underline{\text { Test Sign }}$}

1. Cross Road

2. Right Curve

3. Divided Highway Ends

4. Advance Flagger

5. Bicycle Crossing

6. Pavement Ends

7. No U-Turn

8. Keep Right

9. No Parking

10. No Trucks

11. National Network Route Prohibited

12. Mandatory Seat Belt

13. Hospital

14. Phone

15. RV Sanitary Station

16. Campfire

17. Skating

18. Ranger Station
Foil

Side Road (Right, 90)

Right Reverse Curve

Added Lane

Worker (Construction)

Narrow Bridge

Signal Ahead

No Pedestrians

Straight or Left

No Right Turn

No Bicycles

No Hitchhiking

Keep Left

Picnic

Gas

Diesel

Swimming

Snowmobile

Restrooms 


\section{APPENDIX H}

19 FHWA MODIFIED, REDESIGNED, AND NOVEL SYMBOL SIGNS

\section{$\underline{\text { Modified Signs }}$}

\begin{tabular}{|c|c|c|c|c|}
\hline No. & $\begin{array}{l}\text { Sign } \\
\text { Category } \\
\end{array}$ & $\begin{array}{l}\text { Sign } \\
\text { Name } \\
\end{array}$ & $\begin{array}{l}\begin{array}{c}\text { Sign } \\
\mathrm{Ht} \times \mathrm{Wd}\end{array} \\
\text { inches } \\
(\mathrm{c} \mathrm{m})\end{array}$ & $\underline{\text { Color }}$ \\
\hline 1. & $\operatorname{Reg}(\mathrm{B})$ & Keep Right & $\begin{array}{l}30 \times 24 \\
(76.2)\end{array}$ & Black, White \\
\hline 2. & $\operatorname{Reg}(\mathrm{I})$ & No Parking (P) & $\begin{array}{l}24 \times 24 \\
(61.0)\end{array}$ & $\begin{array}{l}\text { Red, Black, } \\
\text { White }\end{array}$ \\
\hline 3. & Warn(B) & Right Curve & $\begin{array}{l}42.4 \times 42.4 \\
(107.8)\end{array}$ & Yellow, Black \\
\hline 4. & Warn(B) & Cross Road & $\begin{array}{l}42.4 \times 42.4 \\
(107.8)\end{array}$ & Yellow, Black \\
\hline 5 & Warn(I) & Divided Highway Ends & $\begin{array}{l}50.9 \times 50.9 \\
(129.3)\end{array}$ & Yellow, Black \\
\hline 61 & $\mathrm{G} / \mathrm{R}(\mathrm{B})$ & Hospital & $\begin{array}{l}24 \times 24 \\
(61.0)\end{array}$ & Blue, White \\
\hline 7. & $\mathrm{~W}$ a $\mathrm{r} n$ & $\begin{array}{l}\text { Lane Reduction } \\
\text { Transition }\end{array}$ & $\begin{array}{l}50.9 \times 50.9 \\
(129.3)\end{array}$ & Yellow, Black \\
\hline
\end{tabular}

Notes:

$\begin{array}{ll}\text { Warn } & =\text { Warning } \\ \text { Reg } & =\text { Regulatory } \\ \text { G/R } & =\text { Guide/Recreational } \\ \text { (B) } & =\text { Best Category } \\ \text { (I) } & =\text { Intermediate Category } \\ \text { (W) } & =\text { Worst Category } \\ \mathrm{Ht} & =\text { height } \\ \mathrm{Wd} & =\text { width }\end{array}$




\section{$\underline{\text { Redesigned Signs }}$}

\begin{tabular}{|c|c|c|c|c|}
\hline 1. & $\operatorname{Reg}(\mathrm{W})$ & Mandatory Seat Belt & $\begin{array}{l}20 \times 15 \\
(50.80)\end{array}$ & Black, White \\
\hline 2. & Warn(W) & Pavement Ends & $\begin{array}{l}50.9 \times 50.9 \\
(129.3)\end{array}$ & Yellow, Black \\
\hline 3. & Warn $(\mathrm{W})$ & Bicycle Crossing & $\begin{array}{l}42.4 \times 42.4 \\
(107.8)\end{array}$ & Yellow, Black \\
\hline 4. & Warn(I) & Advance Flagger & $\begin{array}{l}50.9 \times 50.9 \\
(129.3)\end{array}$ & Orange, Black \\
\hline 5. & $\mathrm{G} / \mathrm{R}(\mathrm{W})$ & Ranger Station & $\begin{array}{l}24 \times 24 \\
(61.0)\end{array}$ & Brown, White \\
\hline 6. & $\mathrm{G} / \mathrm{R}(\mathrm{I})$ & Campfire & $\begin{array}{l}24 \times 24 \\
(61.0)\end{array}$ & Brown, White \\
\hline 7. & Reg & No Parking (Car) & $\begin{array}{l}24 \times 24 \\
(61.0)\end{array}$ & $\begin{array}{l}\text { Red, Black, } \\
\text { White }\end{array}$ \\
\hline & \multicolumn{4}{|c|}{$\underline{\text { Novel Signs }}$} \\
\hline 1. & Warn & $\begin{array}{l}\text { Reduced Visibility } \\
(107.8)\end{array}$ & $42.4 \times 42.4$ & $\begin{array}{l}\text { Yellow, White, } \\
\text { Black }\end{array}$ \\
\hline 2. & Warn & Cross Winds & $\begin{array}{l}42.4 \times 42.4 \\
(107.8)\end{array}$ & Yellow, Black \\
\hline 3. & Warn & School Bus Stop Ahead & $\begin{array}{l}50.9 \times 50.9 \\
(129.3)\end{array}$ & $\begin{array}{l}\text { Yellow, Red, } \\
\text { Black }\end{array}$ \\
\hline 4. & Warn & Horse Drawn Vehicles & $\begin{array}{l}42.4 \times 42.4 \\
(107.8)\end{array}$ & Yellow, Black \\
\hline 5. & Warn & Truck Entrance & $\begin{array}{l}42.4 \times 42.4 \\
(107.8)\end{array}$ & $\begin{array}{l}\text { Red, Black, } \\
\text { White }\end{array}$ \\
\hline
\end{tabular}




\section{APPENDIX I \\ COMPREHENSION SCORING KEY FOR NOVEL SYMBOLS}

$\mathrm{C}=$ correct; $\mathrm{P}=$ partially correct $; \mathrm{W}=$ possible wrong responses

1. Reduced Visibility

C caution, entering fog; reduced visibility due to fog. (atmospheric conditions) ahead; reduce speed, fog/fog bank ahead.

P (blowing) snow/dust.

W pollution, rain, snow bank, explosion, car wash, bad weather, cloud, car exhaust.

\section{Cross Winds}

C wind, wind gusts, cross winds; strong winds; wind tunnel, windy area.

W slippery road, avalanche area, car overturning.

\section{School Bus Stop Ahead}

C watch out for stopped school bus; school children emerging from school bus ahead.

P school bus area, school bus loading area.

W bus stop, school zone, watch for children on road, school bus route, school crossing.

\section{Horse Drawn Vehicles}

C horse-drawn vehicles/carriage/cart ahead or on road; watch for carriages/carts.

P carriages entering highway; horse and carriage/cart crossing.

W farm area, slow moving vehicles, Amish/Hutterite area, watch for horses/livestock on road, parking area for horse and carriage, race track.

5. Truck Entrance

C truck entrance/entering highway; watch for trucks entering; trucks crossing road ahead (must indicate ENTERING).

P watch for trucks on highway, trucks merging; trucks ahead.

W truck route; truck stop; trucks have right-of-way; truck parking/truck pull-off gravel pit; slow moving vehicles. 


\section{APPENDIX J}

\section{LEGIBILITY SCORING KEY FOR MODIFIED, REDESIGNED, AND NOVEL SYMBOL SIGNS}

\section{Name of Sign}

1. Keep Right

2. No Parking (P)

3. Mandatory Seat Belt

4. Right Curve

5. Cross Road

6. Divided Highway Ends

7. Pavement Ends

8. Bicycle Crossing

9. Flagger Ahead

10. Hospital

11. Ranger Station

\section{Key Features}

Arrow (head) pointing upward

Shaft curves around left obstruction

Traffic island; barrier; thing which obstructs, etc.

Red circle, verify this is seen

Diagonal red slash

Letter $\mathrm{P}$

Seated figure

Seat belt over shoulder and across waist or descriptive equivalent

Arrow (head) curving to the right (not horizontal) Need both direction and arrowhead

Plus; cross; horizontal \& vertical lines

Two curved arrows, up and down, around traffic island; obstruction; barrier

Car headed right

Arrow (head) pointing up

Solid line (road surface) below car on left

Broken dots (rough road) below car on right

Arrow (head) pointing up

Bicycle moving right to left

Road line below bicycle

Lines to the right (behind) of bicycle

Standing figure

Rectangle (flag) upper left

H or descriptive equivalent

Head or face wearing hat (don't need nose or eye)

Building

Flag on roof of building 
12. Campfire

13. Reduced Visibility

14. Cross Winds

15. Lane Drop

16. School Bus Stop Ahead

17. Horse-Drawn Vehicles

18. Truck Entrance

19. No Parking (Car)
Flame (don't need number of flames)

Logs; matches; cigarettes; (or anything which

indicates the cylinderical nature of the objects)

Car going left to right

Cloud; fog; white smoke; puffy cottonball; woolly; soft; vapourous; amorphous quality.

Tipped or tilted car

Line (roadway)

Two lines on right, top line going across/over/around roof of car (don't need direction of car)

Straight vertical line on left

Vertical, curved line on right

Dashed/broken lines

Arrow, curved, going from right to left lane over dashed lines (between lanes)

Arrow (head) pointing up

School bus or vehicle

Figure/ person to left of bus (don't need direction of figure, bag, book)

Road surface line (cue)

Two red dots at both upper corners of bus

* two thresholds measured on this sign:
A) bus alone
B) color of two red dots (stop lights) if different

Horse

Carriage, box and two wheels, wagon, etc.

Vertical line on left

Dashed lines in the middle

Indicate truck

Indicate truck direction: coming onto road, facing left or approaching middle of road, etc.

J-shaped lines above and below truck

Red circle

Red diagonal slash-left top to right bottom

Curb at lower right corner

Car parked at curb

Road surface line 


\section{REFERENCES}

1. Transportation Research Board. Transportation in an aging society: Improving safety and mobility of older persons. TRB Special Report \#218, Volumes 1 and 2, Washington, DC.

2. D.J. Mace. Sign legibility and conspicuity. In Transportation in an Aging Society. Improving Safety and Mobility for Older Persons, Washington DC: Transportation Research Board, Vol. 2, 1988, pp. 270-293.

3. U.S. Department of Transportation, Federal Highway Administration. (1988). Manual on uniform traffic control devices for streets and highways. Washington: Author.

4. S. Rosenbloom. The mobility needs of the older driver. In Transportation in an Aging Society: Improving Safety and Mobility for Older Persons. Vol. 2, 1988, pp. 2 1-71, Washington, DC: Transportation Research Board.

5. R. Huston, and M. Janke. Senior Driver Facts. Report CAL-DMV-RSS-86-82, Sacramento: California Department of Motor Vehicles, 1986.

6. L. Evans. Traffic Safety and the Driver. New York: Van Nostrand Reinholder, 1991.

7. E. Cerrelli. Older drivers: the age factor in traffic safety. NHTSA Technical Report DOT HS 807 402, U. S. Dept. of Transportation, February, 1989.

8. F. X. McKelvey et al. Relationship between driver age and highway accidents. Report MTR-86-008A. Lansing: Michigan Office of Highway Safety Planning, 1987.

9. L.L. Laux and J. Brelsford Jr. Age-related changes in sensory, cognitive, psychomotor and physical functioning in drivers aged 40 to 92 . Washington, DC: AAA Foundation for Traffic Safety, 1990.

10. D. W. Kline, T. J. B. Kline, J. L. Fozard, W. Kosnik, F. Schieber, and R. Sekuler. Vision, aging and driving: the problems of older drivers. Journal of Gerontology: Psychological Sciences, 1992.

11. D. Yee. A survey of the traffic safety needs and problems of drivers age 55 and over. In Needs and Problems of Older Drivers: Survey Results and Recommendations. Falls Church, VA: AAA Foundation for Traffic Safety, 1985, pp. 96-128.

12. T.W. Planek. The aging driver in today's traffic: A critical review. In Aging and highway safety: The Elderly in a mobile society. North Carolina Symposium on Highway Safety, Vol. 7, 1973, Chapel Hill, North Carolina, University of North Carolina, Safety Research Center.

13. T. Maleck. and J. Hummer. Driver age and highway safety. Transportation Research Record, \# 1059, 1986, pp. 6-12. 
14. L. Staplin, K. Lococo, J. Sim, and M. Drapcho, Age differences in a visual information processing capability underlying traffic control device usage. Transportation Research Record \#1244, 1989, pp. 63-72.

15. M. Mackay. Crash protection for older persons. In Transportation in an Aging Society. Improving Safety and Mobility of Older Persons, Vol. 2, 1988, pp. 158-193. Washington, DC: Transportation Research Board.

16. J.T. Duff and D.R. Greig. Traffic Signs in Great Britain. In Proceedings of the International Conference on Highway Sign Symbology, Washington, DC, 1972, pp. 116, 127.

17. M. Kikura. Traffic signs in Japan. In Proceedings of the International Conference on Highway Sign Symbology, Washington, DC, (1972), 28-38.

18. R.J. Jacobs, A.W. Johnston, and B.L. Cole. The visibility of alphabetic and symbolic traffic signs. Australian Road Research, Vol. 5, 1975, pp. 68-86.

19. T.J.B. Kline, L.M. Ghali, D.W. Kline, and S. Brown. Visibility distance of highway signs among young, middle-aged and older observers: icons are better than text. Human Factors, Vol. 32, 1990, pp. 609-619.

20. J.G. Ells and R.E. Dewar. Rapid comprehension of verbal and symbolic traffic sign messages. Human Factors, Vol. 21, 1979, pp. 161-168.

21. R.E. Dewar and H.A. Swanson. Recognition of traffic control signs. Highway Research Record, \# 414, 1972, pp. 16-23.

22. R.W. Brainard, R.J. Campbell and E.H. Elkin. Design and interpretability of road signs. Journal of Applied Psychology, Vol. 45, 1968, pp. 130- 136.

23. A.M. Mackie. A national survey of knowledge of the new traffic signs. RRL Report No. 51, Road Research Laboratory, Crowthorne, 1966.

24. R.E. Dewar. The slash obscures the symbol on prohibitive traffic signs. Human Factors, Vol. 18, 1976, pp. 253-258.

25. R.E. Dewar. Criteria for the design and evaluation of traffic sign symbols. Transportation Research Record, \#1160, 1988, pp. 1-6.

26. R.E. Dewar. Traffic Signs. In D. Oborne (Ed.). International Reviews of Ergonomics. London: Taylor \& Francis, 1989, pp. 65-86.

27. B.L. Cole and S.E. Jenkins. Conspicuity of traffic control devices. Report No. AIR 218-1, Melbourne: Australian Road Research Board, 1982.

28. B.L. Cole and P. Hughes. A field trial of attention and search conspicuity. Human Factors, Vol. 26, 1984, pp. 299-313. 
29. R. Loo. Individual differences and the perception of traffic signs. Human Factors, Vol. 20, 1978, pp. 65-74.

30. M.R. Khavanin and R.N. Schwab. Traffic sign legibility and conspicuity for the older drivers. In Compendium of Technical Papers from the 61st Annual Meeting of the Institute of Transportation Engineers. Milwaukee, September, 1991.

31. A.M. Mackie. Progress in learning the meanings of symbolic traffic signs. RRL Report LR 91, Road Research Laboratories, Crowthorne, Berkshire, England, 1967.

32. A. M. Mackie, and M.H. Higgs. Motorists' understanding of the meanings of traffic signs. In Proceedings of the International Conference on Highway Sign Symbology, Washington, DC, 1972.

33. R. Koppa, and P. Guseman. Public understanding of traffic control devices in Texas. Report \# FHWA/TX-78/05-232-1 F, Texas Transportation Institute, College Station, 1978.

34. M.D. Van der Nest. Recent research and practice on symbol sign communication in South Africa. In Proceedings of the International Conference on Highway Sign Symbology, Washington, DC, 1972.

35. B.N. Lewis, and J.A. Cook. Toward a theory of telling. International Journal of Manmachine Studies, Vol. 1, 1969.

36. R.E. Dewar, and J.G. Ells. Methods for the evaluation of traffic signs. Final Report, Contract 97096, Ministry of Transport, Ottawa, 1974.

37. J.F. Paniati. Legibility and comprehension of traffic sign symbols. Proceedings of the 32nd Annual Meeting of the Human Factors Society, Anaheim, CA, Vol. 1, 1988, pp. 568572.

38. J.F. Paniati. Redesign and evaluation of selected work zone symbols. Transportation Research Record, \# 1213, 1989, pp. 47-55.

39. R. Knoblauch, and M. Pietrucha. Motorists' Comprehension of Regulatory, Warning, and Symbolic Signs. Contract \# DTFH61-83-C-00136, Volume II: Technical Report, Federal Highway Administration, Washington, DC, 1985.

40. M.A. Ogden, K.N. Womack, and J.M. Mounce. Motorist comprehension of signing in urban traffic arterial work zones. Transportation Research Record, \#1281, 1980, pp. 127135 .

41. H.E. Hawkins, K.N. Womack, and J.M. Mounce. Driver comprehension of warning signs. Paper presented at the 72nd Annual Meeting of the Transportation Research Board, Washington, DC, 1993.

42. Anonymous. Wisconsin drivers tested on highway signs. AASHTO Quarterly, October, 1980 , p. 29. 
43. R.E. Dewar, and J.G. Ells. A comparison of three methods for evaluating traffic signs. Transportation Research Record, \#503, 1974b, pp. 38-47.

44. L.L. Avant, K. Brewer, and A. Thieman. Recognition errors among highway signs. Transportation Research Record, \# 1027, 1985, pp. 45-49.

45. R.E. Dewar, J.G. Ells, and G. Mundy, Reaction time as an index of traffic sign perception. Human Factors, Vol. 18, 1976, pp.381-392.

46. L. Whitaker. The effects of words vs. symbols on reaction times to left-right traffic signs Ergonomics, Vol. 22, 1979, pp. 765.

47. K. Roberts, E.W. Lareau, and D. Welch. Perceptual factors and meanings of symbolic information elements. Report No. FHWARD-77-65, Vol. II, Technical Report, Federal Highway Administration, Washington, DC, 1977.

48. J. Mackett-Stout and R.E. Dewar. Evaluation of symbolic public information signs. Human Factors, Vol. 23, 1981, pp. 139-151.

49. P.L. Olson, and A. Bernstein. The nighttime legibility of highway signs as a function of their luminance characteristics. Human Factors, Vol. 21, 1979, pp. 145-160.

50. K. Rumar and A. Ost. The night driving legibility effects of dirt on road signs. Report 164, Department of Psychology, University of Uppsala, Sweden, 1974.

51. M. J. Allen. Vision and Highway Safety. Philadelphia: Chilton Book Co, 1970.

52. R.E. Dewar and W. Berger. Methodology in traffic sign evaluation. In Proceedings of the International Conference on Highway Sign Symbology, Washington, DC, 1972.

53. R.E. Dewar and J.G. Ells. Methods of evaluation of traffic signs. In Information Design. The Design and Evaluation of Signs and Printed Material (R. Easterby and H. Zwaga, eds.), John Wiley, Chichester, England, 1984.

54. P.S. Shapiro, J.E. Upchurch, J. Loewen, and V. Siaurusaitis. Identification of needed traffic control device research. Transportation Research Record, \# 1114, 1987, pp. 11-20.

55. R.S. Easterby and H.J.G. Zwaga. Evaluation of public information symbols ISO tests: 1975. AP Report 60, Applied Psychology Department, University of Aston, Birmingham, 1976.

56. S. Hulbert, J. Beers, and P. Fowler. Motorists' Understanding of Traffic Control Devices. AAA Foundation for Traffic Safety, Falls Church, VA, 1979.

57. D.W. Kline and P. Fuchs. The visibility of symbolic highway signs can be increased among drivers of all ages. Human Factors, Vol. 35, 1993, pp. 25-34. 
58. L. Staplin, K. Lococo, J. Sim. Traffic control design elements for accommodating drivers with diminished capacity. Volume II. Final technical report. FHWA-RD-90-055, Federal Highway Administration, Washington, DC, 1990.

59. D.W. Kline. Aging and the visibility of highway signs: A new look through older eyes. Washington, DC: AAA Foundation for Traffic Safety, 1991.

60 F. Schieber, L.M. Ghali, and D.W. Kline. Aging and the visibility of symbolic highway signs: a new look through older eyes. Transportation Research Record, (in press).

61. A.P. Ginsburg. Visual information processing based on spatial filters constrained by biological data. (Report No. AMRL-TR-78-129). Springfield, VA: National Technical Information Service, 1978.

62. F. Schieber. Optimizing the visual environment to the older eye: 2-D Fourier models of spatial vision in action. The Gerontologist, Vol. 27, 1987, 166A.

63. J.G. Daugman and D.M. Kammen. Pure orientation filtering: A scale-invariant imageprocessing tool for perception research and data compression. Behavior Research Methods, Instruments, \& Computers, Vol. 18, 1986, pp. 559-564.

64. I.L. Bailey and J.E. Sheedy. Visual screening for driver licensure. In Transportation in an Aging Society: Improving Safety and Mobility for Older Persons, Vol. 2, pp. 294-324, Transportation Safety Board, National Research Council, Washington DC, 1988.

65. J. Cerella. Aging and information processing rate. In J.E. Birren, and K.W. Schaie (Eds.), Handbook of the Psychology of Aging (3rd. ed., pp. 201-221), San Diego: Academic Press, 1990.

66. J.L. Fozard. Vision and hearing in aging. In J.E. Birren and K.W. Schaie (Eds.), Handbook of the Psychology of Aging (3rd. ed., pp. 150-170). Academic Press Inc: San Diego, 1990.

67. D.F. Hultsch and R.A. Dixon. Learning and memory in aging. In J.E. Birren and K.W. Schaie (Eds.), Handbook of the Psychology of Aging (3rd. ed., pp. 258-274). San Diego: Academic Press, 1990.

68. D.W. Kline and F. Schieber. Vision and aging. In J.E. Birren and K.W. Schaie (Eds.), Handbook of the Psychology of Aging (2nd ed., 296-331), Van Nostrand Reinholder, New York, 1985.

69. J.M. McDowd and J.E. Birren. Aging and attentional processes. In J.E. Birren and K.W. Schaie (Eds.), Handbook of the Psychology of Aging (3rd. ed., pp. 222-233). San Diego: Academic Press, 1990.

70. C. Owsley and M.E. Sloane. Vision and aging. In F. Boller and J. Grafman (Eds.), Handbook of the Neuropsychology (Vol. 4) (pp. 229-249). Amsterdam: Elsevier, 1990. 
71. F. Schieber. Vision assessment technology and screening older drivers: Past practices and emerging techniques. In Transportation in an Aging Society: Improving Safety and Mobility for Older Persons (Vol. 2, pp. 325-378). Washington DC: Transportation Research Board, 1988.

72. F. Schieber, J.L. Fozard, S. Gordon-Salant and J.M. Weiffenbach. Optimizing sensation and perception in older adults. International Journal of Industrial Ergonomics, Vol. 7, 1991, pp. 133-162.

73. R. Sekuler, D.W. Kline, and K. Dismukes (Eds.). Aging and Human Visual Function, New York: Alan R. Liss, 1982.

74. Q.W. Spirduso, and P.G. MacRae. Motor performance and aging. In J.E. Birren and K.W. Schaie (Eds.), Handbook of the Psychology of Aging (3rd. ed., pp. 183-200). San Diego: Academic Press, 1990.

75. W.A. Weale. Aging and vision. Vision Research, Vol. 26, 1986, pp. 1507-1512.

76. D. Shinar and F. Schieber. Visual requirements for safety and mobility of older drivers. Human Factors, Vol. 33, 1991, pp. 507-519.

77. D. Shinar, E.D. McDowell, N.J. Rackoff, and T.H. Rockwell. Field dependence and driver visual search behavior. Human Factors, Vol. 20, 1978, pp. 553-560.

78. I.E. Loewenfeld. Pupillary changes related to age. In H.S. Thompson (Ed.), Topics in Neuro-ophthalmology. Baltimore: Williams and Wilkins, 1979.

79. F.S. Said and R.A. Weale. The variation with age of the spectral transmissivity of the living human crystalline lens. Gerontologica, Vol. 3, 1959, pp. 213-231.

80. R.A. Weale. Retinal illumination and age. Transactions of the Illuminating Engineering Society, Vol. 26, 1961, pp. 95-100.

81. E. Wolf. Glare and age. Archives of Ophthalmology, Vol. 64, 1960, pp. 502-514.

82. M.G. Block and W.M. Rosenblum. MTF measurements on the human lens. Journal of the Optical Society of America, Vol. 4, 1987, p. 18.

83. R. Bruckner. Longitudinal research in the eye. Gerontologia Clinica, Vo1.9, 1967, pp. 8795 .

84. N.M. Simonelli. The dark focus of the human eye and its relationship to age and visual defect. Human Factors, Vol. 25, 1983, pp. 85-92.

85. C.L. Elworth, C. Larry and F.V. Malmstrom. Age, degraded viewing environments, and the speed of accommodation. Aviation, Space and Environmental Medicine, Vol. 57, 1986, pp. 54-58. 
86. M.L. Boice, M.A. Tinker and D.G. Paterson. Color vision and age. American Journal of Psychology, Vol. 61, 1948, pp. 520-526.

87. A. Chapanis. Relationships between age, visual acuity and color vision. Human Biology, Vol. 22, 1950, pp. 1-33.

89. L.M. Dalderup and M.L.C. Fredericks. Color sensitivity in older age. Journal of the American Geriatric Society, Vol. 17, 1969, pp. 388-390.

90. J.G. Gilbert. Age changes in color matching. Journal of Gerontology, Vol. 12, 1957, pp. 210-215.

92. N.S. Gittings, J.L. Fozard, and N.W. Shock. Age changes in stereopsis, visual acuity, and color vision. Paper presented at the meeting of the Gerontological Society of America, Washington, DC, November, 1987.

92. K. Knoblauch, F. Saunders, M. Kusuda, R. Hynes, M. Podgor, K.E. Higgins, and F.M. de Monasterio. Age and illuminance effects in the Farnsworth-Munsell 100-hue test. Applied Optics, Vol. 26, 1987, pp. 1441-1448.

93. P.R. Boyce, and R.H. Simons. Hue discrimination and light sources. Lighting Research and Technology, Vol. 9, 1977, pp. 125-140.

94. A. Eisner, S.A. Fleming, M.L. Klein, and W.M. Mauldin. (1987). Sensitivities in older eyes with good acuity: cross-sectional norms. Investigative Ophthalmology and Visual Science, Vol. 28, 1987, pp. 1824-1831.

95. J.A. Sharpe and T.O. Sylvester. Effects of aging on horizontal smooth pursuit. Investigative Ophthalmology and Visual Science, Vol. 17, 1978, pp. 465-468.

96. W. Chamberlain. Restriction in upward gaze with advancing age. American Journal of Ophthalmology, Vol. 71, 1971, pp. 341-346.

97. T. Warabi, M. Kase and T. Kato. Effect of aging on the accuracy of visually guided saccadic eye movement. Annals of Neurology, Vol. 16, 1984, pp. 449-454.

98. W. Kosnik, D.W. Kline, H. Fikre, and R. Sekuler. Ocular fixation control as a function of age and exposure duration. Psychology and Aging, Vol. 2, 1987, pp. 302-305.

99. S.P. Sturgis, and D.J. Osgood. Effects of glare and background luminance on visual acuity and contrast sensitivity: implications for night vision testing. Human Factors, Vol. 24, 1982, pp. 347-360.

100. J.E. Birren, M.W. Bick and C. Fox. Age changes in the light threshold of the dark adapted eye. Journal of Gerontology, Vol. 3, 1948, pp. 267-271.

101. R.A. McFarland and M.B. Fisher. Alterations in dark adaptation as a function of age. Journal of Gerontology, Vol. 10, 1955, pp. 424-428. 
102. R.A. McFarland, R.G. Domey, A.B. Warren and D.C. Ward. Dark adaptation as a function of age: I. A statistical analysis. Journal of Gerontology, Vol. 15, 1960, pp. 149154.

103. R.A. Weale. On the eye. In A.T. Welford and J.E. Birren (Eds.), Behavior, Aging, and the Nervous System (pp. 307-325). Springfield, IL: Charles C. Thomas, 1965.

104. J.E. Birren and N.W. Shock. Age changes in rate and level of visual dark adaptation. Journal of Applied Physiology, Vol. 2, 1950, pp. 407-4111.

1051 R.A. Weale, R. A. The Aging Eye. London: Lewis, 1963.

106. R.A. Weale, Senile ocular changes, cell death, and vision. In R. Sekuler, D.W. Kline, and K. Dismukes (Eds.), Aging and Human Visual Function. New York: Alan R. Liss, 1982, pp. 161-171.

107. R.L. Gregory. Neurological noise as a factor in aging. Proceedings of the Fourth Congress of the International Association of Gerontology, Merano, 1957, pp. 314-324.

108. A.M. Prestrude, K. Levenick and K. Woody. The effect of age upon the detection of short wavelength light. Tower International Techno Medical Institute Journal of Life Sciences, Vol. 3, 1973, pp. 101-105.

109. J.H. Carter. The effects of aging on selected visual functions: color vision, glare sensitivity, field of vision, and accommodation. In R. Sekuler, D.W. Kline, and K. Dismukes K. (Eds.), Aging and Human Visual Function. New York: Alan R. Liss, 1982, pp. $121-130$.

110. N.H. Pulling, E. Wolf, S.P. Sturgis, D.R. Vaillancourt, and J.J. Dolliver. Headlight glare resistance and driver age. Human Factors, Vol. 22, 1980, pp. 103-112.

111. A. Burg. Light sensitivity as related to age and sex. Perceptual and Motor Skills, Vol. 24, 1967, pp. 1279-1288.

112. V. Reading. Yellow and white headlamps glare and age. Transactions of the Illuminating Engineering Society, Vol. 31, 1966, pp. 108-121.

113. P.L. Olson and M. Sivak. Glare from automobile rear-vision mirrors. Human Factors, Vol. 26, 1984, pp. 269-282.

114. D.B. Elliott. Contrast sensitivity decline with aging: a neural or optical phenomenon? Ophthalmic and Physiological Optics, Vol. 7, 1987, pp. 415-419.

115. F. Schieber and M.J. Williams. The effects of age and glare upon spatiotemporal contrast thresholds. The Gerontologist, Vol. 30, 1990, p. 71. 
116. C.E. Feree, G. Rand, and E.F. Lewis. The effect of increase of intensity of light on the visual acuity of presbyopic and non-presbyopic eye. Transactions of the Illuminating Engineering Society, Vol. 29, 1934, pp. 296-313.

117. H.C. Weston. The effect of age and illumination upon visual performance with close sights. British Journal of Ophthalmology, Vol. 32, 1948, pp. 645-653.

118. D. Rice and B. Jones. Vision screening of drivers license renewal applicants. Salem, Oregon: Department of Transportation, Motor Vehicle Division, 1984.

119. O. W. Richards. Effects of luminance and contrast on visual acuity, ages 16-90 years. American Journal of Optometry and Physiological Optics, Vol. 54, 1977, pp. 178-184.

120. L. Vola, C. Cornu, P. Carvel, P. Gastaud, and J. Leid. L'age et les acuities visuelles photopiques et mesopiques. Journale Francaise Ophtalmolgie, Vol. 6, 1983, pp. 473-479.

121. J.F. Sturr and H.A. Taub. Performance of young and older drivers on a static acuity test under photopic and mesopic luminance conditions. Human Factors, Vol. 32, 1990, pp. 1-8.

122. A. Van Meeteren and J.J. Vos. Resolution and contrast sensitivity at low luminances. Vision Research, Vol. 12, 1972, pp. 825-833.

123. D. W. Kline, F. Schieber, L.C. Abusamra, A.C. Coyne. Age, the eye, and the visual channels: contrast sensitivity and response speed. Journal of Gerontology, Vol. 38, 1983, pp. 211-216.

124. J.D. Morrison, and C. McGrath. Assessment of the optical contributions to the age-related deterioration in vision. Quarterly Journal of Experimental Physiology, Vol. 70, 1985, pp. 249-269.

125. C. Owsley, R. Sekuler, and D. Siemsen. Contrast sensitivity throughout adulthood. Vision Research, Vol. 23, 1983, pp. 689-699.

126. M.E. Sloane, C. Owsley, S.L. Alvarez. Aging, senile miosis and spatial contrast sensitivity at low luminance. Vision Research, Vol. 28, 1988, pp. 1235-1246.

127. D.W. Evans and A.P. Ginsburg. Contrast sensitivity predicts age-related differences in highway-sign discriminability. Human Factors, 27, 1985, pp. 637-642.

128. C. Owsley and M.E. Sloane. Contrast sensitivity, acuity, and the perception of 'real world' targets. British Journal of Ophthalmology, Vol. 71, 1987, pp. 791-796.

129. C.A. Johnson and J.L. Keltner. Incidence of visual field loss and its relationship to driving performance. Archives of Ophthalmology, Vol. 101, 1983, pp. 371-375.

130. A. Burg. Visual acuity as measured by static and dynamic tests. Journal of Applied Psychology, Vol. 50, 1966, pp. 460-466. 
131. A. Burg and S. Hulbert. Dynamic visual acuity as related to age, sex, and static acuity. Journal of Applied Psychology, Vol. 45, 1961 , pp. 111-116.

132. T. Farrimond. Visual and auditory performance variations with age: some implications. Australian Journal of Psychology, Vol. 19, 1967, pp. 193-201.

133. V.M. Reading. Visual resolution as measured by dynamic and static tests. Pflugers Archives: European Journal of Physiology, Vol. 333, 1972, p. 1726.

134. C.T. Scialfa, P.M. Garvey, K. W. Gish, L.M. Deering, H.W. Leibowitz, and C.C. Goebel. Relationships among measures of static and dynamic sensitivity. Human Factors, Vol. 30, 1988, pp. 677-687.

135. E.J. Ludvigh and J. W. Miller. Study of visual acuity during the pursuit of moving test objects. I. Introduction. Journal of the Optical Society of America, Vol. 48, 1958, pp. 799802 .

136. G.M. Long, and R.F. Crambert. The nature and basis of age-related changes in dynamic visual acuity. Psychology and Aging, Vol. 5, 1990, pp. 138-143.

137. E.J. Ludvigh. Visual acuity while one is viewing a moving object. Archives of Ophthalmology, Vol. 42, 1949, pp. 14-22.

138. C. W. Tyler. Specific deficits of flicker sensitivity in glaucoma and ocular hypertension. Investigative Ophthalmology and Visual Science, Vol. 20, 198 1, pp. 204-2 12.

139. M.J. Mayer, C.B. Kim, A. Svingos, and A. Glucs. Fovea1 flicker sensitivity in healthy aging eyes: compensating for pupil variation. Journal of the Optical Society of America, Vol. 5, 1988, pp. 2201-2209.

140. T. Kuyk, and M. Wesson. Changes in flicker sensitivity with age. Investigative Ophthalmology and Visual Science (supplement), Vol. 30, 1989, pp. 314.

141. F.L. Royer and G.C. Gilmore. Spatiotemporal factors and developmental changes in visual processes. Bulletin of the Psychonomic Society, Vol. 23, 1985, pp. 404-406.

142. C. W. Tyler. (1989). Two processes control variations in flicker sensitivity over the lifespan. Journal of the Optical Society of America A, 6, 481-490.

143. W. Kosnik, R. Sekuler and D.W. Kline. Self-reported problems of current and former older drivers. Human Factors, Vol. 32, 1990. pp. 597-608.

144. J.M. McDowd, and F.I.M. Craik. Effects of aging and task difficulty on divided attention performance. Journal of Experimental Psychology: Human Perception and Performance, Vol. 14, 1988, pp. 764-769.

145. A.W. Kazniak, P.M. Keyl and M.S. Albert. Dementia and older drivers. Human Factors, Vol. 35, 1991, pp. 527-538. 
146. L.L. Wright and J.W. Elias. Age differences in the effects of perceptual noise. Journal of Gerontology, Vol. 43, 1979, pp. 704-708.

147. D. J. Plude and W.J. Hoyer. Attention and performance: Identifying and localizing age deficits. In N. Charness (Ed.), Aging and Performance (pp. 47-99). New York: Wiley, 1985.

148. R. Sekuler and K. Ball. Visual localization and practice. Journal of the Optical Society of America (Section A), Vol. 3, 1986, pp. 864-867.

149. C.T. Scialfa, D. W. Kline, and B.J. Lyman. Age differences in target identification as a function of retinal location and noise level: examination of the useful field of view. Psychology and Aging, Vol. 2, 1987, pp. 14-19.

150. K. Ball, C. Owsley, and B. Beard. Clinical visual perimetry underestimates peripheral field problems in older adults. Clinical Vision Sciences, Vol. 5, 1990, pp. 113-125.

151. C. Owsley, K. Ball, M.E. Sloane, D.L. Roenker, and J.R. Bruni. Visual/cognitive correlates of vehicle accidents in older drivers. Psychology and Aging, Vol. 6, 1991, pp. 403-415.

152. J.E. Birren. Age changes in speeded behavior: its central nature and physiological correlates. In A.T. Welford and J.E. Birren (Eds.), Behavior, Aging, and the Nervous System , pp. 191-216. Springfield, IL: Thomas, 1965.

153. M.J. Stones and A. Kozma. Cross-sectional, longitudinal, and secular age trends in athletic performance. In M.L. Howe and C.J. Brainard (Eds.), Cognitive Development in Adulthood; Progress in Cognitive Development Research. New York: Springer, 1989.

154. K.W. Schaie. Intellectual development in adulthood. In J.E. Birren and K.W. Schaie (eds.), Handbook of the Psychology of Aging (3rd. ed., pp. 291-309). San Diego: Academic Press, 1990.

155. T.A. Salthouse. Competence and cognitive expertise. In J.E. Birren and K.W. Schaie (eds.), Handbook of the Psychology of Aging (3rd. ed., pp. 31-3 19). San Diego: Academic Press, 1990.

156. J.D. Blaschke, M.E. Dennis and F.T. Creasy. Physical and psychological effects of alcohol and other drugs. ITE Journal, Vol. 57, 1987, issue 6, pp. 33-38.

157. J.A. Hicks. An evaluation of the effects of sign brightness on the sign reading behavior of alcohol-impaired drivers. Human Factors, 1976, Vol. 18, pp.45-52.

158. P.L. Olson, M. Sivak and J.C. Egan. Variables influencing the nighttime legibility of highway signs. Report No. UMTRI-83-36, Ann Arbor, MI: University of Michigan Transportation Research Canter, 1983. 
159. B.R. Cooper. Comprehension of traffic signs by drivers and non-drivers. TRRL Research Report \#167, Crowthorne: Transportation and Road Research Laboratory, 1989.

160. R.R. Mourant and G.D. Langoff. Luminance specifications for automobile instrument panels. Human Factors, Vol. 18, 1976, pp. 71-84.

161. M. Sivak, P. Olson, and L.A. Pastalan. Effects of driver age on nighttime legibility of traffic signs. Human Factors, Vol. 23, 1981) pp. 59-64.

162. S. Hulbert and P. Fowler. Motorists' understanding of traffic control devices II. Falls Church, VA: AAA Foundation for Traffic Safety, 1980.

163. R.W. Allen, Z. Parseghain and P.C.A. Van Valkenburg. Simulator evaluation of age effects on symbol sign recognition. Proceedings of the Human Factors Society 24th Annual Meeting, 1980.

164. P.T. Caimey. Understanding traffic control devices. Special Report \# 44, Australian Road Research Board, South Vermont, Australia, 1989.

165. L. Laux and D. Mayer. Road sign comprehension: age and sex effects. Paper presented at the Annual Meeting of the Transportation Research Board, Washington, DC, 1991.

166. N.L. Mergler and T. Zandi. Adult age differences in speed and accuracy of matching verbal and pictorial signs. Educational Gerontology, Vol 9, pp. 73-85.

167. D.F. Halpern. Age differences in response time to verbal and symbolic traffic signs. Experimental Aging Research, Vol. 10, 1984, pp. 201-204.

168. C.F. Shoptaugh and L.A. Whitaker. Verbal response times to directional traffic signs embedded in photographic street scenes. Human Factors, Vol. 26, 1984, pp. 235-244.

169. L.A. Whitaker \& S. Stacey. Response times to left and right directional signs. Human Factors, Vol. 23, 1981 447-452.

170. D.G. Pitts. (1982). The effects of aging on selected visual functions: Dark adaptation, visual acuity, stereopsis, and brightness contrast. In R. Sekuler, D. Kline, \& K. Dismukes (Eds.), Aging and Human Visual Function (pp. 131-159). New York: Alan R. Liss, Inc.

171. J.L. Keltner, \& C.A. Johnson. (1987) Visual function, driving safety, and the older. Ophthalmology, 94, 1180-1188.

172. H.T. Zwahlen, X. Hu, M. Sunkara, \& L.M. Duffus. (1991). Recognition of traffic sign symbols in the field during daytime and nighttime. Proceedings of the Human Factors Society 35th Annual Meeting (pp. 1058-1062).

173. C.T. Scialfa, R.A. Tyrell, P.M. Garvey, L.M. Deering, H. W. Leibowitz, \& C.C. Goebel (1988). Age differences in Vistech near contrast sensitivity. American Journal of Ophthalmology and Physiological Optics, 65, 951-956. 
174. K. Ball, B. Beard, D. Roenker, R. Miller, \& D. Griggs. (1988). Age and visual search: Expanding the useful field of view. Journal of the Optical Society of America, 5, 22102219. 